\title{
Access to Difluoromethylated Arenes by Pd-Catalyzed Reaction of Arylboronic Acids with Bromodifluoroacetate
}

Zhang Feng, Qiao-Qiao Min, and Xingang Zhang*

Key Laboratory of Organofluorine Chemistry, Shanghai Institute of Organic Chemistry, Chinese Academy of Sciences, 345 Lingling Road, Shanghai 200032, China

xgzhang@mail.sioc.ac.cn 


\section{List of Contents}

1) Screening for Pd-Catalyzed Difluoromethylation of Aryl Boronic Acid 1a with Bromodifluoroacetate 2 (Table S1-S8) ................................................ 33

2) Kinetic Studies of the Reaction of $\mathbf{1 a}$ with $\mathbf{2}$ or $\mathbf{V}$ (Scheme S1, Figure S1)......................S8

3) Reaction of Compound $\mathbf{4}$ or $\mathbf{4}^{\prime}$ with Hydroquinone under Standard Reaction Conditions (Scheme

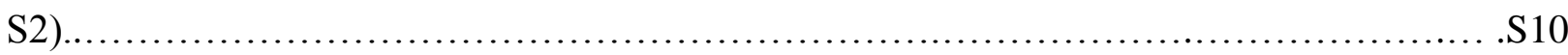

4) The Role of Hydroquinone for the Reaction (Scheme S3) ..................................

5) The Role of Fe(acac) $)_{3}$ for the Reaction (Schemes S4-S5, Figures S2-S3) ...................S11

6) Identification of Difluorocarbene: Reaction of $\mathbf{1 a}$ with $\mathbf{V}$ under Standard Reaction Conditions in

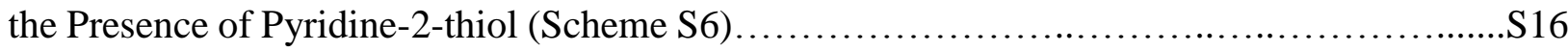

7) Reaction of $\mathbf{1 a}$ with $\mathbf{V}$ in the Presence of Palladium Complex VI (Scheme S7). S17

8) Cross-Coupling Reaction between $1 \mathrm{a}$ and 2 in the Presence of $\mathrm{Pd}\left(\mathrm{PPh}_{3}\right)_{4}$ with or without Fe(acac $)_{3}$ (Scheme S8)

9) X-Ray Photoelectron Spectroscopy (XPS) Analysis of the Reaction (Scheme S9, Figure S4). S18

10) General Procedure for Pd-Catalyzed Difluoromethylation of Aryl Boronic Acids with

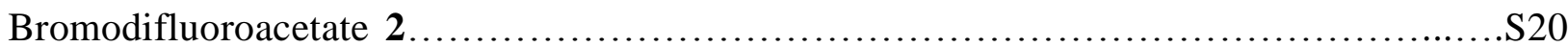

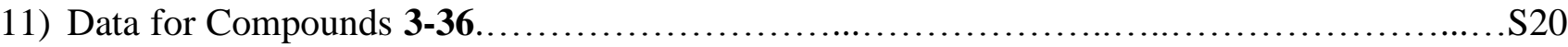

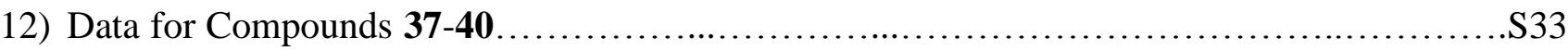

13) Copies of ${ }^{19} \mathrm{~F}$ NMR and ${ }^{13} \mathrm{C}$ NMR spectra of compound V ........................S36

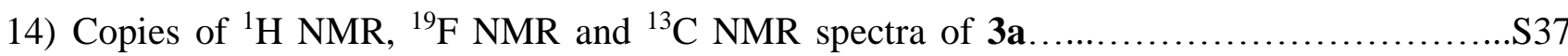

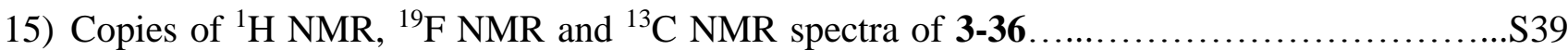

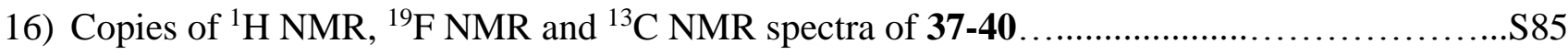


General information: ${ }^{1} \mathrm{H}$ NMR and ${ }^{13} \mathrm{C}$ NMR spectra were recorded on a Bruker AM400 and AM500 spectrometer. ${ }^{19} \mathrm{~F}$ NMR was recorded on a Bruker $\mathrm{AM} 400$ spectrometer $\left(\mathrm{CFCl}_{3}\right.$ as an external standard and low field is positive). Chemical shifts $(\delta)$ are reported in ppm, and coupling constants $(J)$ are in $\operatorname{Hertz}(\mathrm{Hz})$. The following abbreviations were used to explain the multiplicities: $\mathrm{s}=$ singlet, $\mathrm{d}=\mathrm{doublet}$, $\mathrm{t}=$ triplet, $\mathrm{q}=$ quartet, $\mathrm{m}=$ multiplet, $\mathrm{br}=$ broad. $\mathrm{NMR}$ yield was determined by ${ }^{19} \mathrm{~F}$ NMR using fluorobenzene as an internal standard before working up the reaction.

Materials: All reagents were used as received from commercial sources, unless specified otherwise. Anhydrous $\mathrm{K}_{2} \mathrm{CO}_{3}$ was purchased from Alfa. DMF, DMSO, and DCE were distilled under reduced pressure from $\mathrm{CaH}_{2}$. 1,4-Dioxane and xylene were distilled from sodium and benzophenone immediately before use.

Table S1. Pd-Catalyzed Reaction of Arylboronic Acid 1a with Bromodifluoroacetate 2 in the Presence of Different Oxidants. ${ }^{a}$
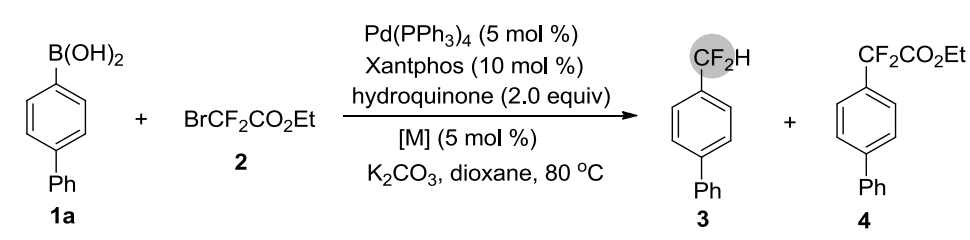

\begin{tabular}{ccc}
\hline entry & oxidant & $\mathbf{3 / 4}$ yield $(\%)^{b}$ \\
\hline 1 & $\mathrm{Cu}(\mathrm{OTf})_{2}$ & Trace $/ 79$ \\
2 & $\mathrm{Cu}(\text { acac })_{2}$ & $17 / 43$ \\
3 & $\mathrm{Cu}(\mathrm{OAc})_{2}$ & $6 / 61$ \\
4 & $\mathrm{Co}(\text { acac })_{3}$ & $32 / 12$ \\
5 & $\mathrm{Fe}(\text { acac })_{3}$ & $39 / 43$ \\
$\mathbf{6}^{\mathbf{c}}$ & $\mathbf{F e}(\mathbf{a c a c})_{3}$ & $\mathbf{4 8 / 4 6}$ \\
\hline
\end{tabular}

${ }^{a}$ Reaction conditions (unless otherwise specified): $\mathbf{1 a}\left(0.3 \mathrm{mmol}, 1.0\right.$ equiv), $\mathbf{2}$ (2.0 equiv), $\mathrm{K}_{2} \mathrm{CO}_{3}(4.0$ equiv), dioxane $(2 \mathrm{~mL}), 80^{\circ} \mathrm{C}, 24 \mathrm{~h} .{ }^{b}$ Determined by ${ }^{19} \mathrm{~F}$ NMR using fluorobenzene as an internal standard. ${ }^{c} \mathrm{Fe}(\mathrm{acac})_{3}(3.5 \mathrm{~mol} \%)$. 
Table S2. Screening of Palladium Sources. ${ }^{a}$

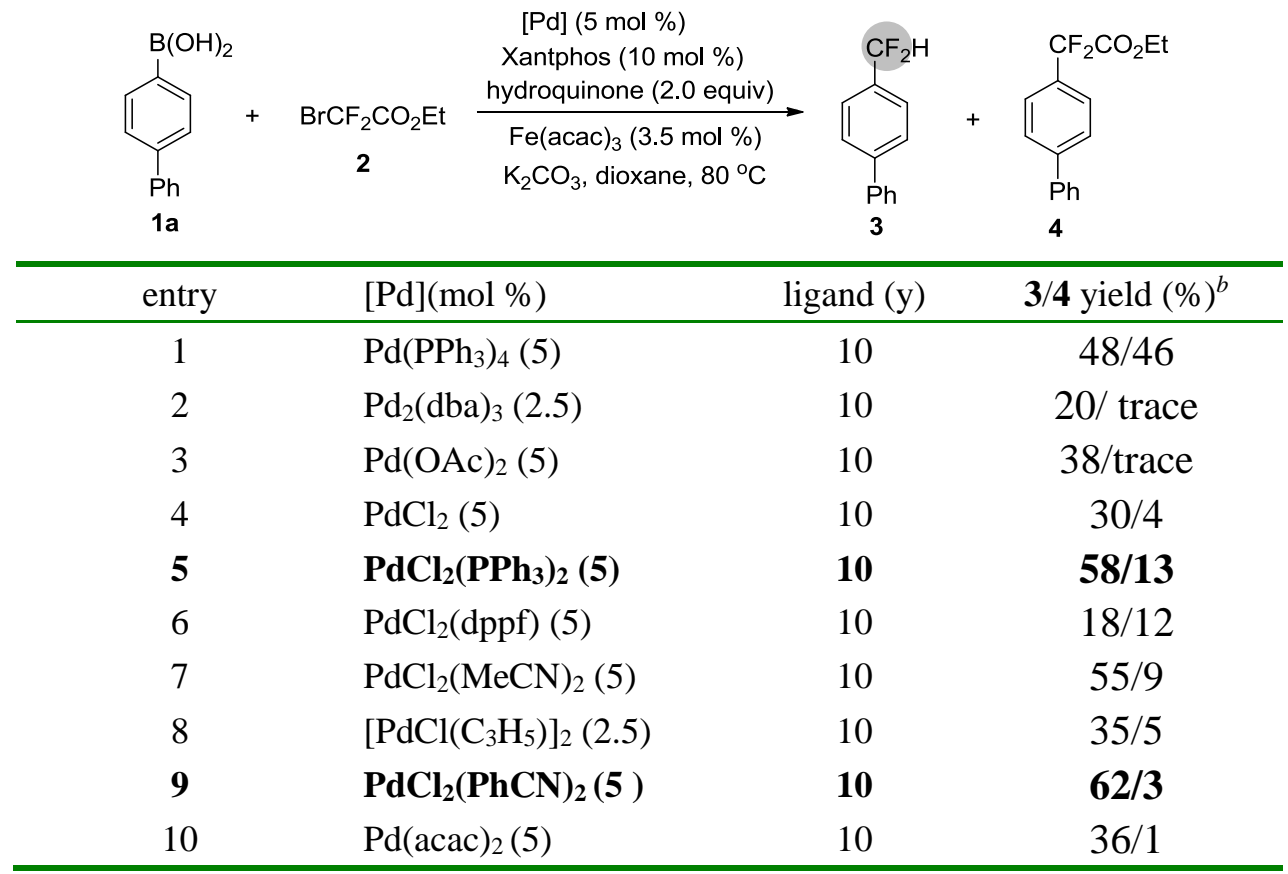

${ }^{a}$ Reaction conditions (unless otherwise specified): $\mathbf{1 a}\left(0.3 \mathrm{mmol}, 1.0\right.$ equiv), $\mathbf{2}$ (2.0 equiv), $\mathrm{K}_{2} \mathrm{CO}_{3}$ (4.0 equiv), dioxane $(2 \mathrm{~mL}), 80^{\circ} \mathrm{C}, 24 \mathrm{~h} .{ }^{b}$ Determined by ${ }^{19} \mathrm{~F}$ NMR using fluorobenzene as an internal standard.

Table S3. Screening of Bases. ${ }^{a}$
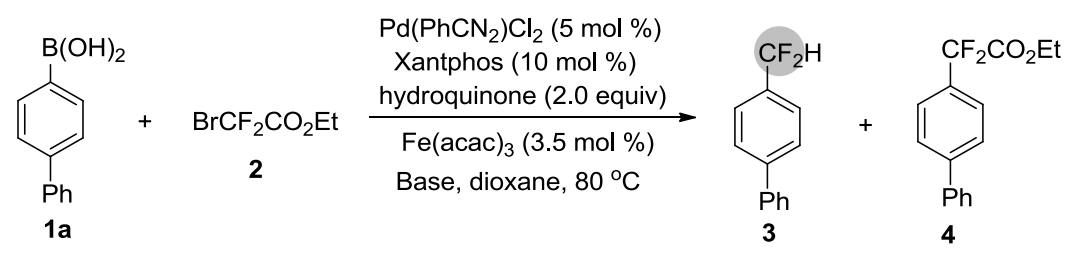

\begin{tabular}{ccc}
\hline entry & base (equiv) & 3a/4a yield $(\%)^{b}$ \\
\hline 1 & $\mathrm{~K}_{2} \mathrm{CO}_{3}(4.0)$ & $62 / 3$ \\
2 & $\mathrm{~K}_{3} \mathrm{PO}_{4}(4.0)$ & $16 / 11$ \\
3 & $\mathrm{KOAc}(4.0)$ & Trace/4 \\
4 & $\mathrm{C} O \mathrm{OAc}(4.0)$ & $\mathrm{Trace} / 1$ \\
5 & $\mathrm{NaOAc}(4.0)$ & $\mathrm{nd} / 2$ \\
6 & $\mathrm{Na}_{2} \mathrm{CO}_{3}(4.0)$ & $\mathrm{nd} / 17$ \\
7 & $\mathrm{~K}_{2} \mathrm{CO}_{3}(3.5)$ & $50 / 6$ \\
8 & $\mathrm{~K}_{2} \mathrm{CO}_{3}(3.0)$ & $49 / 7$ \\
9 & $\mathrm{~K}_{2} \mathrm{CO}_{3}(2.5)$ & $46 / 10$ \\
12 & $\mathrm{~K}_{2} \mathrm{CO}_{3}(2.0)$ & $10 / 23$ \\
13 & $\mathrm{~K}_{2} \mathrm{CO}_{3}(1.5)$ & $\mathrm{Trace} / 3$ \\
\hline
\end{tabular}

${ }^{a}$ Reaction conditions (unless otherwise specified): $1 \mathbf{a}\left(0.3 \mathrm{mmol}, 1.0\right.$ equiv), 2 (2.0 equiv), dioxane $(2 \mathrm{~mL}), 80{ }^{\circ} \mathrm{C}, 24$ h. ${ }^{b}$ Determined by ${ }^{19} \mathrm{~F}$ NMR using fluorobenzene as an internal standard. 
Table S4. Screening of Ligands. ${ }^{a}$

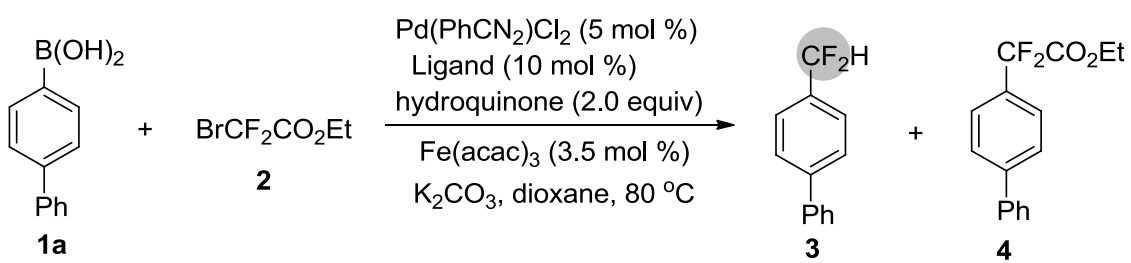<smiles>CC1(C)c2cccc(P)c2Oc2c(-c3ccccc3)cccc21</smiles>

XantPhos<smiles>Pc1ccccc1Oc1cccc(P)c1-c1ccccc1</smiles>

DpePhos

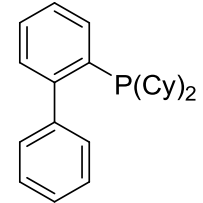

CyJohnPhos<smiles>[Y6]1c2ccccc2c2ccccc12</smiles>

MePhos<smiles>CN(C)c1ccccc1-c1ccccc1</smiles>

DavePhos<smiles>CCPc1ccccc1-c1c(C(C)C)cc(C(C)C)cc1C(C)C</smiles>

XPhos<smiles>COc1ccccc1-c1c(OC)cccc1OC</smiles>

SPhos

\begin{tabular}{ccc}
\hline entry & ligand & $\mathbf{3 a} / \mathbf{4 a}$ yield $(\%)^{b}$ \\
\hline 1 & Xantphos & $62 / 3$ \\
2 & DpePhos & $32 / \mathrm{nd}$ \\
3 & dppe & $\mathrm{nd} / \mathrm{nd}$ \\
4 & dppp & $\mathrm{nd} / \mathrm{nd}$ \\
5 & dppb & $\mathrm{nd} / \mathrm{nd}$ \\
6 & dppf & $\mathrm{nd} / \mathrm{nd}$ \\
7 & BINAP & $\mathrm{nd} / 3$ \\
8 & CyJohnPhos & trace/trace \\
9 & MePhos & $\mathrm{nd} / \mathrm{nd}$ \\
10 & DavePhos & $\mathrm{nd} / \mathrm{nd}$ \\
11 & XPhos & $\mathrm{nd} / \mathrm{nd}$ \\
12 & SPhos & $\mathrm{nd} / \mathrm{nd}$ \\
$13^{c}$ & Xantphos & $45 / 2$ \\
$14^{d}$ & Xantphos & $\mathbf{6 3 / 2}$
\end{tabular}

${ }^{a}$ Reaction conditions (unless otherwise specified): $\mathbf{1 a}\left(0.3 \mathrm{mmol}, 1.0\right.$ equiv), 2 (2.0 equiv), $\mathrm{K}_{2} \mathrm{CO}_{3}(4.0$ equiv), dioxane $(2 \mathrm{~mL}), 80{ }^{\circ} \mathrm{C}, 24 \mathrm{~h} .{ }^{b}$ Determined by ${ }^{19} \mathrm{~F}$ NMR using fluorobenzene as an internal standard. ${ }^{C}$ Xantphos $(5 \mathrm{~mol} \%)$. ${ }^{d}$ Xantphos (7.5 mol \%). 
Table S5. Screening of Solvents. ${ }^{a}$

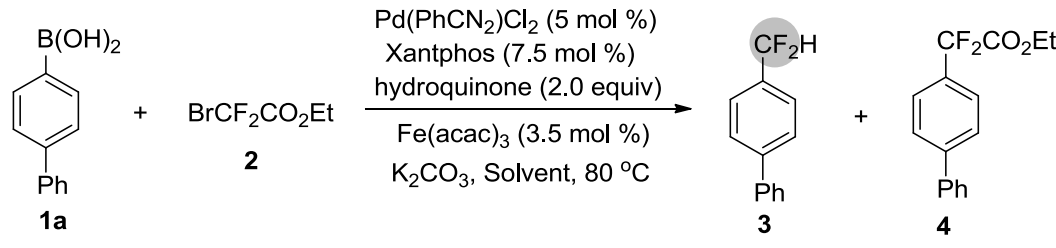

\begin{tabular}{ccc}
\hline entry & solvent & $\mathbf{3 a} / \mathbf{4 a}$ yield $(\%)^{b}$ \\
\hline $\mathbf{1}$ & dioxane & $\mathbf{6 3 / 1}$ \\
2 & DMSO & $\mathrm{nd} / \mathrm{nd}$ \\
3 & Dimethoxyethane & $3 / \mathrm{nd}$ \\
4 & DMF & $\mathrm{nd} / \mathrm{nd}$ \\
5 & Xylene & $\mathrm{nd} / 40$ \\
6 & DCE & trace/nd \\
7 & Diglyme & $2 / \mathrm{nd}$ \\
\hline
\end{tabular}

${ }^{a}$ Reaction conditions (unless otherwise specified): $\mathbf{1 a}\left(0.3 \mathrm{mmol}, 1.0\right.$ equiv), $\mathbf{2}$ (2.0 equiv), $\mathrm{K}_{2} \mathrm{CO}_{3}$ (4.0 equiv), solvent $(2 \mathrm{~mL}), 80{ }^{\circ} \mathrm{C}, 24 \mathrm{~h} .{ }^{b}$ Determined by ${ }^{19} \mathrm{~F}$ NMR using fluorobenzene as an internal standard.

Table S6. Screening of Different Amount of Styrene. ${ }^{a}$

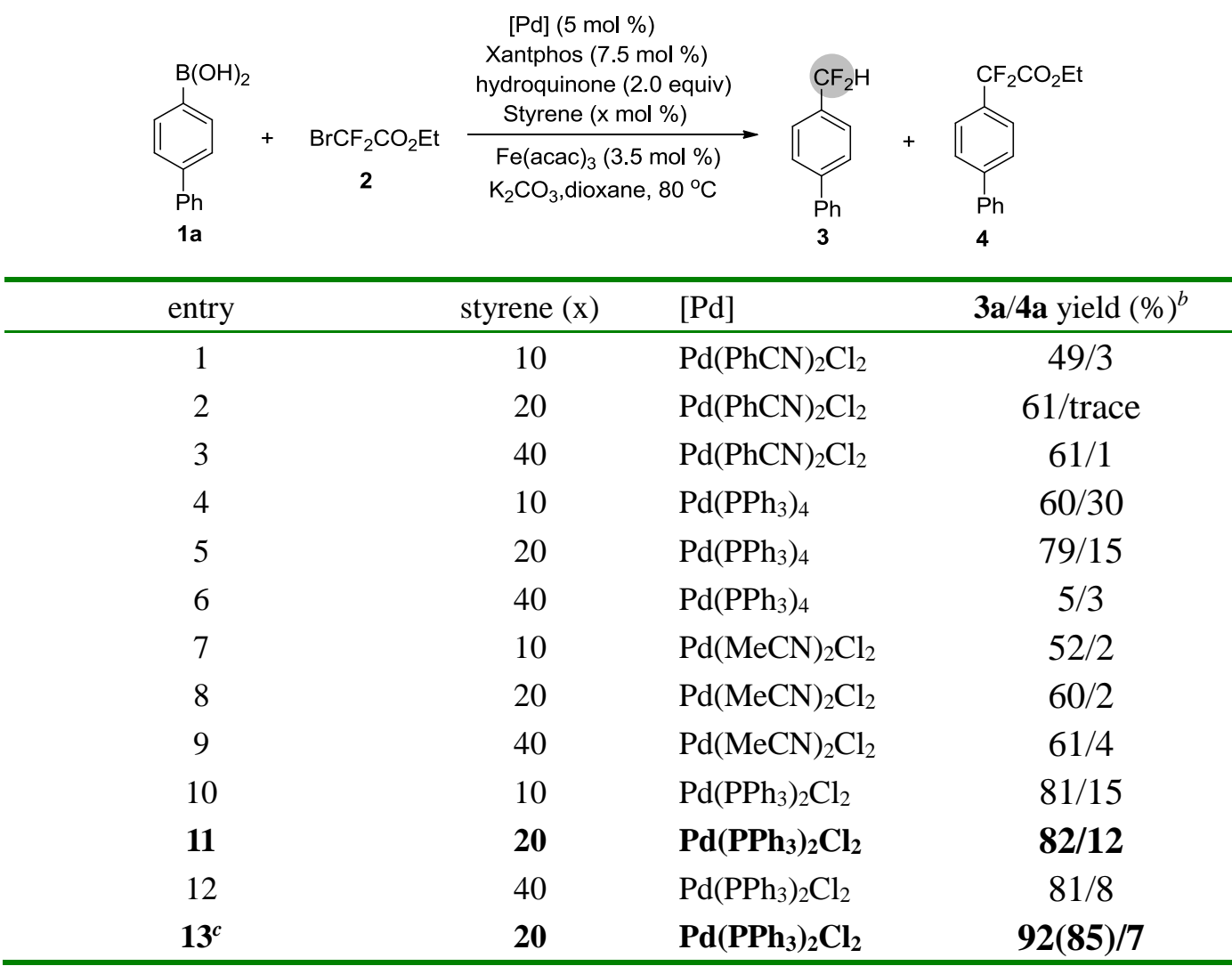

${ }^{a}$ Reaction conditions (unless otherwise specified): $\mathbf{1 a}\left(0.3 \mathrm{mmol}, 1.0\right.$ equiv), $\mathbf{2}$ (2.0 equiv), [Pd] (5 mol \%), $\mathrm{K}_{2} \mathrm{CO}_{3}$ (4.0 equiv), dioxane $(2 \mathrm{~mL}), 80{ }^{\circ} \mathrm{C}, 24 \mathrm{~h} .{ }^{b}$ Determined by ${ }^{19} \mathrm{~F}$ NMR using fluorobenzene as an internal standard and number in parenthesis is isolated yield.. ${ }^{c} .5 \mathrm{~mL}$ of dioxane was used. 
Table S7. Screening of Phenols. ${ }^{a}$
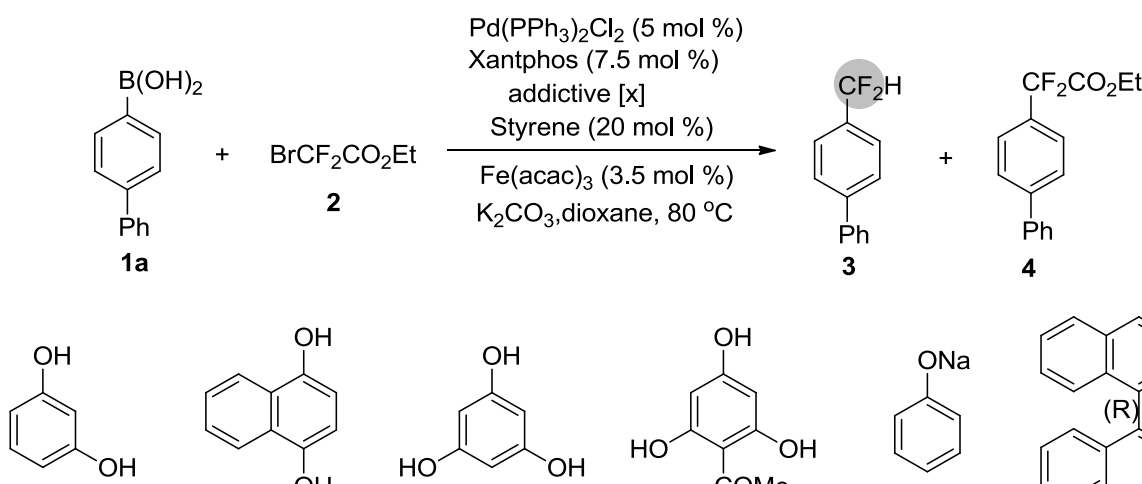<smiles>Oc1cccc2c(O)cccc12</smiles>

B<smiles>Oc1cc(O)cc(O)c1</smiles>

C<smiles>COc1cc(O)cc(O)c1O</smiles>

D<smiles>[O-]c1ccccc1</smiles><smiles>Oc1ccc2ccccc2c1-c1c(O)ccc2ccccc12</smiles>

$\mathbf{F}$

\begin{tabular}{cccc}
\hline entry & additive & {$[\mathrm{x}$ equiv $]$} & $\mathbf{3 a} / \mathbf{4 a}$ yield $(\%)^{b}$ \\
\hline 1 & $\mathbf{A}$ & $(2.0$ equiv $)$ & $\mathrm{nd} / \mathrm{nd}$ \\
2 & $\mathbf{B}$ & $(2.0$ equiv $)$ & $18 /$ trace \\
3 & $\mathbf{C}$ & $(2.0$ equiv $)$ & $2 / \mathrm{nd}$ \\
4 & $\mathbf{D}$ & $(2.0$ equiv $)$ & $3 /$ trace \\
5 & $\mathbf{E}$ & $(2.0$ equiv $)$ & $10 / 9$ \\
6 & $\mathbf{F}$ & $(2.0$ equiv $)$ & $10 /$ trace \\
$\mathbf{7}$ & Hydroquinone & $(\mathbf{2 . 0}$ equiv $)$ & $\mathbf{9 2 / 7}$ \\
8 & Hydroquinone & $(1.5$ equiv $)$ & $54 / 16$ \\
9 & hydroquinone & $(1.0$ equiv $)$ & $42 / 20$ \\
10 & hydroquinone & $(0.5$ equiv $)$ & $25 / 36$ \\
\hline
\end{tabular}

${ }^{a}$ Reaction conditions (unless otherwise specified): $\mathbf{1 a}\left(0.3 \mathrm{mmol}, 1.0\right.$ equiv), $\mathbf{2}$ (2.0 equiv), $\mathrm{K}_{2} \mathrm{CO}_{3}$ (4.0 equiv), dioxane $(2.5 \mathrm{~mL}), 80^{\circ} \mathrm{C}, 24 \mathrm{~h} .{ }^{b}$ Determined by ${ }^{19} \mathrm{~F}$ NMR using fluorobenzene as an internal standard. 
Table S8. Control Experiments of Pd-Catalyzed Reaction of Arylboronic Acid 1a with Bromodifluoroacetate. ${ }^{a}$

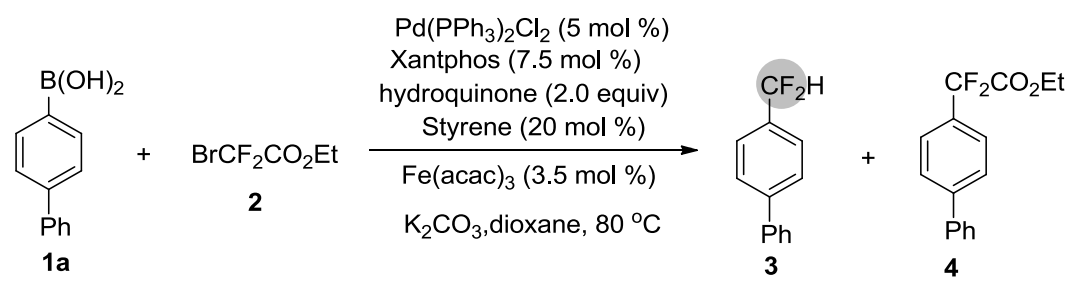

\begin{tabular}{ccccccc}
\hline entry & {$[\mathrm{Fe}]$} & {$[\mathrm{Pd}]$} & ligand & additive & alkene & $\mathbf{3 / 4}$ yield $(\%)^{b}$ \\
\hline 1 & none & none & none & hydroquinone & styrene & $\mathrm{nd} / \mathrm{nd}$ \\
2 & $\mathrm{Fe}(\mathrm{acac})_{3}$ & none & none & hydroquinone & styrene & $\mathrm{nd} / \mathrm{nd}$ \\
3 & none & $\mathrm{Pd}\left(\mathrm{PPh}_{3}\right)_{2} \mathrm{Cl}_{2}$ & none & hydroquinone & styrene & $\mathrm{nd} / 7$ \\
4 & none & none & Xantphos & hydroquinone & styrene & $\mathrm{nd} / \mathrm{nd}$ \\
5 & $\mathrm{Fe}(\mathrm{acac})_{3}$ & none & Xantphos & hydroquinone & styrene & $\mathrm{nd} / \mathrm{nd}$ \\
6 & $\mathrm{Fe}(\mathrm{acac})_{3}$ & $\mathrm{Pd}\left(\mathrm{PPh}_{3}\right)_{2} \mathrm{Cl}_{2}$ & none & hydroquinone & styrene & $\mathrm{nd} / 6 \%$ \\
7 & none & $\mathrm{Pd}\left(\mathrm{PPh}_{3}\right)_{2} \mathrm{Cl}_{2}$ & Xantphos & hydroquinone & styrene & $45 /$ trace \\
8 & none & $\mathrm{Pd}\left(\mathrm{PPh}_{3}\right)_{2} \mathrm{Cl}_{2}$ & Xantphos & hydroquinone & none & $65 /$ trace \\
9 & $\mathrm{Fe}(\mathrm{acac})_{3}$ & ${\mathrm{Pd}\left(\mathrm{PPh}_{3}\right)_{2} \mathrm{Cl}_{2}}_{2}$ & Xantphos & hydroquinone & none & $45 / 39$ \\
10 & $\mathrm{Fe}(\mathrm{acac})_{3}$ & $\mathrm{Pd}\left(\mathrm{PPh}_{3}\right)_{2} \mathrm{Cl}_{2}$ & Xantphos & none & styrene & $\mathrm{nd} / 33 \%$ \\
$\mathbf{1 1}$ & $\mathbf{F e}(\mathbf{a c a c})_{\mathbf{3}}$ & $\mathbf{P d}\left(\mathbf{P P h}_{3}\right)_{2} \mathbf{C l}_{\mathbf{2}}$ & Xantphos & hydroquinone & styrene & $\mathbf{9 2 / 7}$ \\
\hline
\end{tabular}

${ }^{a}$ Reaction conditions (unless otherwise specified): $\mathbf{1 a}\left(0.3 \mathrm{mmol}, 1.0\right.$ equiv), 2 (2.0 equiv), $\mathrm{K}_{2} \mathrm{CO}_{3}(4.0$ equiv), dioxane $(2.5 \mathrm{~mL}), 80{ }^{\circ} \mathrm{C}, 24 \mathrm{~h} .{ }^{b}$ Determined by ${ }^{19} \mathrm{~F}$ NMR using fluorobenzene as an internal standard.

\section{Mechanistic Studies}

\section{Scheme S1. Kinetic Studies of the Reaction of 1a with 2 or V.}

a)

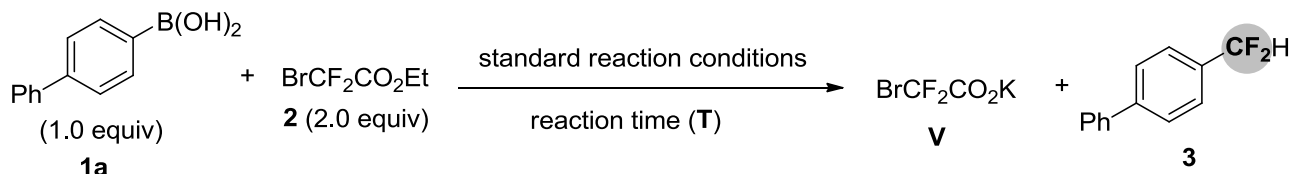

\begin{tabular}{|c|c|c|c|c|}
\hline Entry & Time & $\mathbf{2}$ (Conversion) & V (Yield) & $\mathbf{3}$ (Yield) \\
\hline 1 & $10 \mathrm{~min}$ & $29 \%$ & $23 \%$ & $0 \%$ \\
\hline 2 & $30 \mathrm{~min}$ & $64 \%$ & $60 \%$ & $1 \%$ \\
\hline 3 & $1.0 \mathrm{~h}$ & $80 \%$ & $76 \%$ & $3 \%$ \\
\hline 4 & $1.5 \mathrm{~h}$ & $99 \%$ & $79 \%$ & $13 \%$ \\
\hline 5 & $2.0 \mathrm{~h}$ & $99 \%$ & $76 \%$ & $20 \%$ \\
\hline 6 & $2.5 \mathrm{~h}$ & $99 \%$ & $72 \%$ & $25 \%$ \\
\hline 7 & $3.0 \mathrm{~h}$ & $99 \%$ & $63 \%$ & $32 \%$ \\
\hline 8 & $4.0 \mathrm{~h}$ & $100 \%$ & $56 \%$ & $39 \%$ \\
\hline 9 & $5.0 \mathrm{~h}$ & $100 \%$ & $49 \%$ & $49 \%$ \\
\hline 10 & $6.0 \mathrm{~h}$ & $100 \%$ & & \\
\hline
\end{tabular}


b)

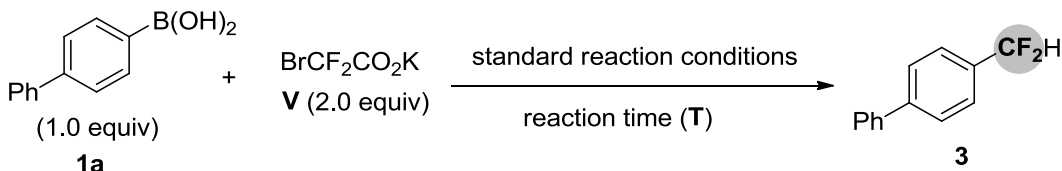

$1 \mathrm{a}$

\begin{tabular}{|c|c|c|c|}
\hline Entry & Time & V( Recovery) & 3 (Yield) \\
\hline 1 & $10 \mathrm{~min}$ & $90 \%$ & $0 \%$ \\
\hline 2 & $30 \mathrm{~min}$ & $87 \%$ & $3 \%$ \\
\hline 3 & $1.0 \mathrm{~h}$ & $83 \%$ & $7 \%$ \\
\hline 4 & $1.5 \mathrm{~h}$ & $78 \%$ & $10 \%$ \\
\hline 5 & $2.0 \mathrm{~h}$ & $72 \%$ & $15 \%$ \\
\hline 6 & $2.5 \mathrm{~h}$ & $70 \%$ & $17 \%$ \\
\hline 7 & $3.0 \mathrm{~h}$ & $66 \%$ & $22 \%$ \\
\hline 8 & $4.0 \mathrm{~h}$ & $58 \%$ & $27 \%$ \\
\hline 9 & $5.0 \mathrm{~h}$ & $50 \%$ & $33 \%$ \\
\hline 10 & $6.0 \mathrm{~h}$ & $43 \%$ & $44 \%$ \\
\hline
\end{tabular}
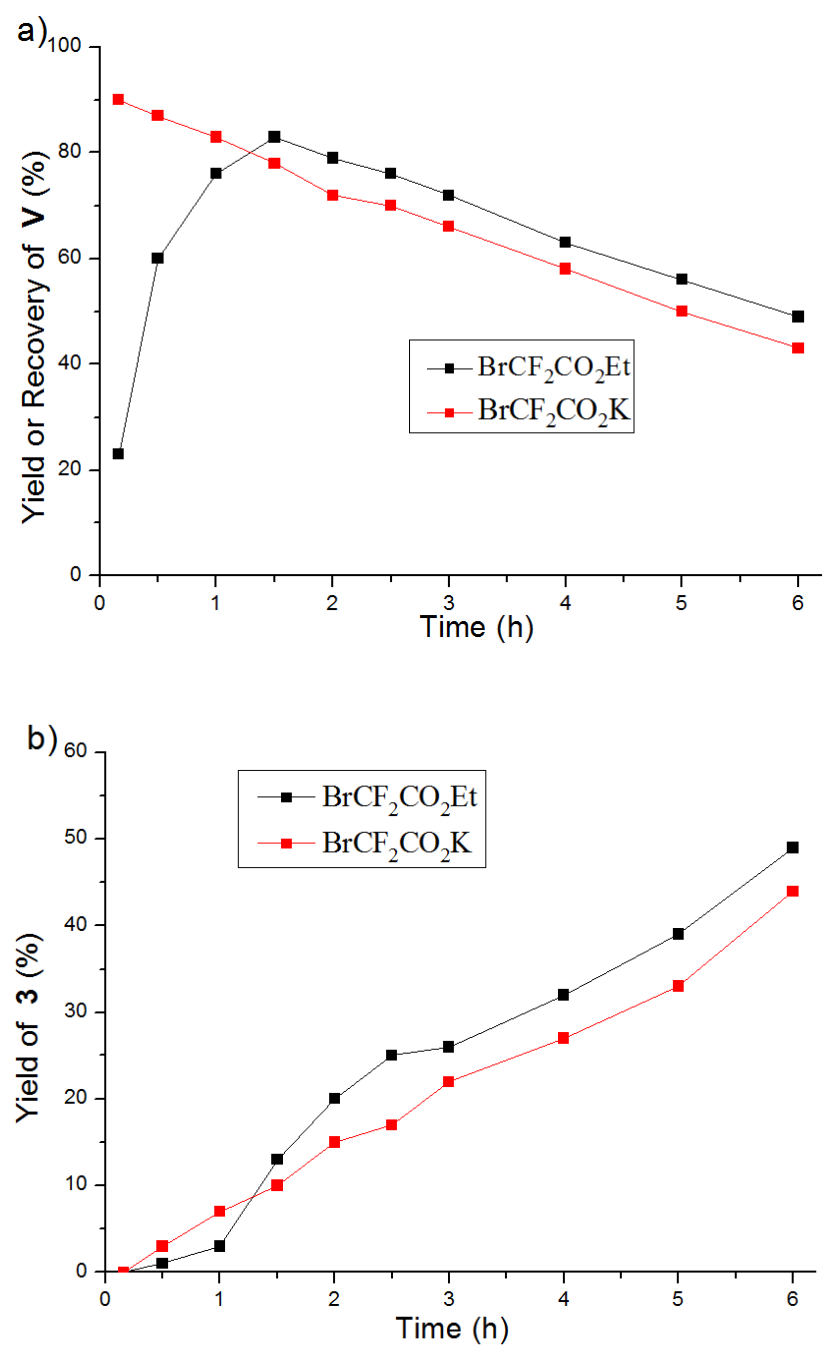

Figure S1. a) Yield (black line) or Recovery (red line) of $\mathbf{V}$ and $\mathbf{b}$ ) Yield of $\mathbf{3}$ with $\mathrm{BrCF}_{2} \mathrm{CO}_{2} \mathrm{Et}$ (black line) or $\mathrm{BrCF}_{2} \mathrm{CO}_{2} \mathrm{~K}$ (red line) as a Starting Material. 
Procedure: To a $25 \mathrm{~mL}$ of Schlenk tube were added anhydrous $\mathrm{K}_{2} \mathrm{CO}_{3}$ (powder, 4.0 equiv), and hydroquinone (2.0 equiv) in glove box, followed by $\mathrm{Fe}(\mathrm{acac})_{3}(3.5 \mathrm{~mol} \%), \mathrm{PdCl}_{2}\left(\mathrm{PPh}_{3}\right)_{2}(5 \mathrm{~mol} \%)$, Xantphos (7.5 mol \%), aryl boronic acid 1a $(0.3 \mathrm{mmol})$, styrene $(20 \mathrm{~mol} \%)$, ethyl bromodifluoroacetate 2 (2.0 equiv) or $\mathrm{BrCF}_{2} \mathrm{CO}_{2} \mathrm{~K}$ (2.0 equiv), and fresh distilled dioxane (2.5 mL). The reaction mixture was heated to $80{ }^{\circ} \mathrm{C}$ (oil bath). The reaction was monitored by ${ }^{19} \mathrm{~F}$ NMR using fluorobenzene as an internal standard.

Note: Attempt to isolation of pure $\mathbf{V}$ failed. The structure of $\mathbf{V}$ was characterized by ${ }^{19} \mathrm{~F} \mathrm{NMR},{ }^{13} \mathrm{C}$ NMR, IR, and MS from a mixture of $\mathbf{V}$ and hydroquinone. All of these data are consistence with the data of $\mathrm{BrCF}_{2} \mathrm{CO}_{2} \mathrm{~K}$. It also should be mentioned that the ${ }^{1} \mathrm{H}$ NMR spectroscopy of the mixture of $\mathbf{V}$ and hydroquinone only showed the spectrum of hydroquinone. Therefore, compound $\mathbf{V}$ was assigned to be $\mathrm{BrCF}_{2} \mathrm{CO}_{2} \mathrm{~K}$. Compound V: ${ }^{19} \mathrm{~F}$ NMR (376 MHz, DMSO) $\delta-52.5$ (s, $\left.2 \mathrm{~F}\right) ;{ }^{13} \mathrm{C}$ NMR (100 MHz, DMSO) $\delta 160.9(\mathrm{t}, J=22.9 \mathrm{~Hz}), 117.2(\mathrm{t}, J=325.2 \mathrm{~Hz}) . \mathrm{IR}$ (thin film) $v_{\max } 1687,1385,1143 \mathrm{~cm}^{-1}$; MS (ESI): $m / z(\%), 172.8\left(\mathrm{M}^{+}-\mathrm{K}^{+}\right), 174.9\left(\mathrm{M}^{+}-\mathrm{K}^{+}\right)$.

\section{Scheme S2. Reaction of Compound 4 or 4' with Hydroquinone under Standard Reaction Conditions}

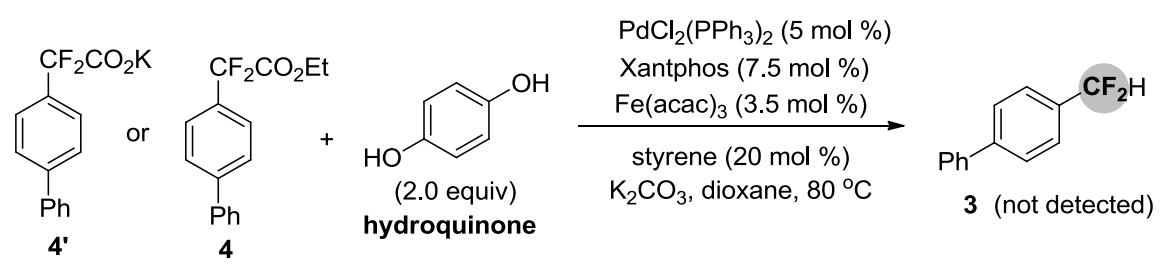

Procedure: To a $25 \mathrm{~mL}$ of Schlenk tube were added anhydrous $\mathrm{K}_{2} \mathrm{CO}_{3}$ (powder, 4.0 equiv), and hydroquinone (2.0 equiv) in glove box, followed by $\mathrm{Fe}(\mathrm{acac})_{3}(3.5 \mathrm{~mol} \%), \mathrm{PdCl}_{2}\left(\mathrm{PPh}_{3}\right)_{2}(5 \mathrm{~mol} \%)$, Xantphos (7.5 mol \%), compound 4 or 4' (0.3 mmol, 1.0 equiv), styrene (20 mol \%) and fresh distilled dioxane $(2.5 \mathrm{~mL})$. The reaction mixture was heated to $80{ }^{\circ} \mathrm{C}$ (oil bath). After stirring for $24 \mathrm{~h}$, the reaction was cooled to room temperature. ${ }^{19} \mathrm{~F}$ NMR spectroscopy showed no compound $\mathbf{3}$ was formed. 


\section{The Role of Hydroquinone for the Reaction}

\section{Scheme S3. The Roles of Hydroquinone for the Reaction}

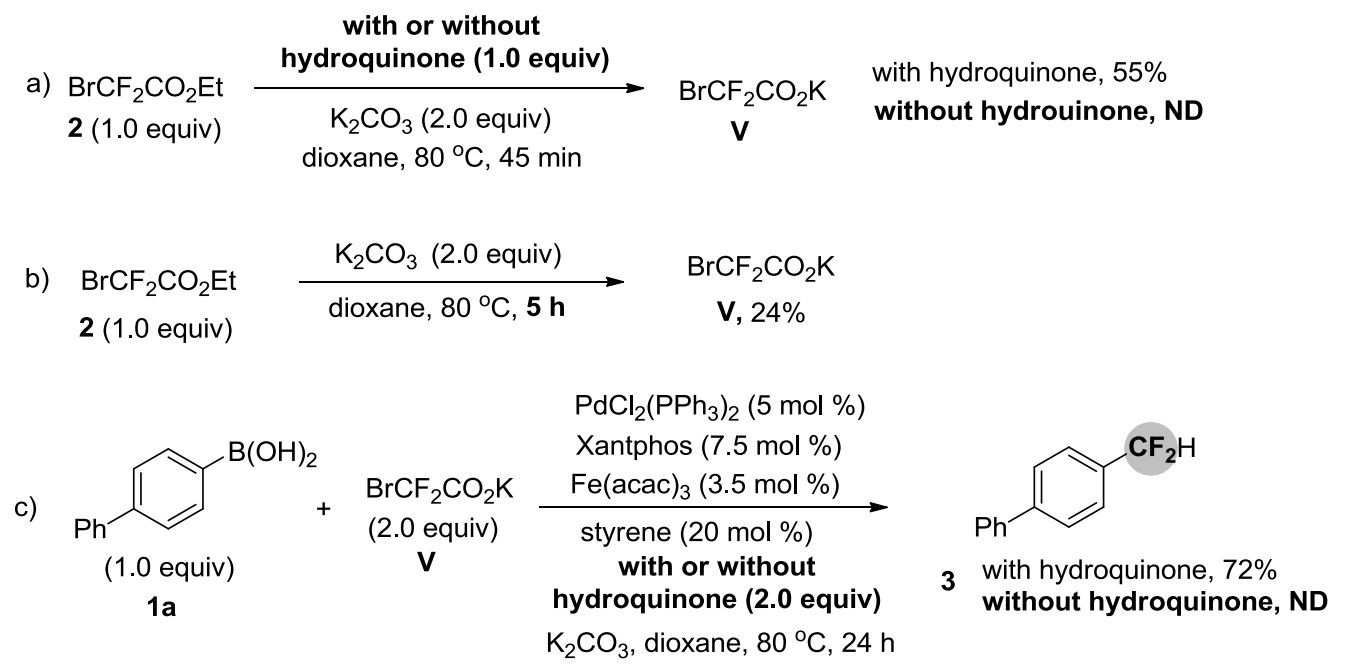

Procedure for the reaction of bromodifluoroacetate 2 with $\mathrm{K}_{2} \mathrm{CO}_{3}$ in the presence of hydroquinone: To a $25 \mathrm{~mL}$ of Schlenk tube were added anhydrous $\mathrm{K}_{2} \mathrm{CO}_{3}$ (powder, 2.0 equiv), hydroquinone (1.0 equiv), ethyl bromodifluoroacetate 2 (1.0 equiv, $0.6 \mathrm{mmol}$ ) and fresh distilled dioxane $(2.5 \mathrm{~mL})$ under $\mathrm{N}_{2}$. The reaction mixture was heated to $80{ }^{\circ} \mathrm{C}$ (oil bath). The reaction was monitored by ${ }^{19} \mathrm{~F}$ NMR using fluorobenzene as an internal standard.

\section{The Role of $\mathrm{Fe}(\mathrm{acac}) 3$ for the Reaction}

The comparsions of reaction of $\mathbf{1 a}$ with $\mathbf{2}$ or $\mathbf{V}$ in the presence of hydroquinone with or without $\mathrm{Fe}(\mathrm{acac})_{3}$ and styrene (Scheme S5-S6 and Figure S2-S3) revealed that the iron species could facilitate the transformation of $\mathbf{V}$ into final product $\mathbf{3}$.

Scheme S4. Cross-Coupling Reaction between Arylboronic Acid 1a and Bromodifluoroacetate 2 with or without $\mathrm{Fe}(\mathrm{acac})_{3}$ and Styrene.

With $\mathrm{Fe}(\mathrm{acac})_{3}$ and Styrene

a)

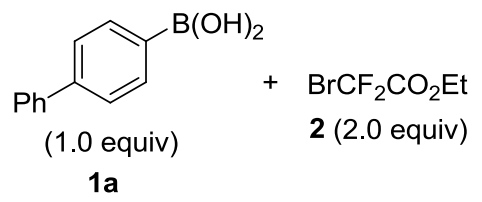

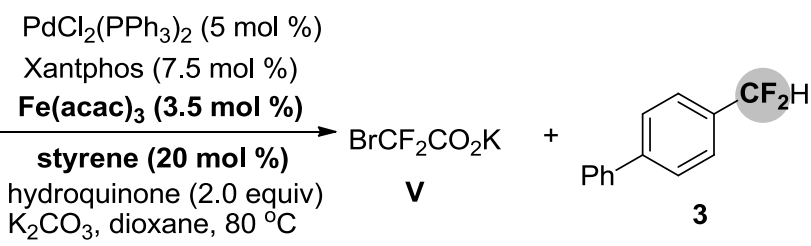

reaction time $(\mathbf{T})$ 


\begin{tabular}{|c|c|c|c|c|}
\hline Entry & Time & $\mathbf{2}$ (Conversion) & $\mathbf{V}$ (Yield) & $\mathbf{3}$ (Yield) \\
\hline 1 & $10 \mathrm{~min}$ & $29 \%$ & $23 \%$ & $0 \%$ \\
\hline 2 & $30 \mathrm{~min}$ & $64 \%$ & $60 \%$ & $1 \%$ \\
\hline 3 & $1.0 \mathrm{~h}$ & $80 \%$ & $76 \%$ & $3 \%$ \\
\hline 4 & $1.5 \mathrm{~h}$ & $99 \%$ & $83 \%$ & $13 \%$ \\
\hline 5 & $2.0 \mathrm{~h}$ & $99 \%$ & $79 \%$ & $20 \%$ \\
\hline 6 & $2.5 \mathrm{~h}$ & $99 \%$ & $76 \%$ & $25 \%$ \\
\hline 7 & $3.0 \mathrm{~h}$ & $99 \%$ & $72 \%$ & $26 \%$ \\
\hline 8 & $4.0 \mathrm{~h}$ & $100 \%$ & $63 \%$ & $32 \%$ \\
\hline 9 & $5.0 \mathrm{~h}$ & $100 \%$ & $56 \%$ & $39 \%$ \\
\hline 10 & $6.0 \mathrm{~h}$ & $100 \%$ & $49 \%$ & $49 \%$ \\
\hline
\end{tabular}

\section{Without $\mathrm{Fe}(\mathrm{acac})_{3}$ and Styrene}

b)<smiles>O=C(O)c1ccc(Br)cc1</smiles>

1a

$+\mathrm{BrCF}_{2} \mathrm{CO}_{2} \mathrm{Et}$ 2 (2.0 equiv)
$\mathrm{PdCl}_{2}\left(\mathrm{PPh}_{3}\right)_{2}(5 \mathrm{~mol} \%)$

Xantphos (7.5 mol \%)

hydroquinone (2.0 equiv) $\mathrm{BrCF}_{2} \mathrm{CO}_{2} \mathrm{~K}+$ $\mathrm{K}_{2} \mathrm{CO}_{3}$, dioxane, $80^{\circ} \mathrm{C}$

reaction time $(\mathbf{T})$

\begin{tabular}{|c|c|c|c|c|}
\hline Entry & Time & $\mathbf{2}$ (Conversion) & $\mathbf{V}$ (Yield) & $\mathbf{3}$ (Yield) \\
\hline 1 & $10 \mathrm{~min}$ & $40 \%$ & $60 \%$ & $0 \%$ \\
\hline 2 & $30 \mathrm{~min}$ & $93 \%$ & $91 \%$ & $0 \%$ \\
\hline 3 & $1.0 \mathrm{~h}$ & $98 \%$ & $93 \%$ & $2 \%$ \\
\hline 4 & $1.5 \mathrm{~h}$ & $100 \%$ & $92 \%$ & $10 \%$ \\
\hline 5 & $2.0 \mathrm{~h}$ & $100 \%$ & $70 \%$ & $14 \%$ \\
\hline 6 & $2.5 \mathrm{~h}$ & $100 \%$ & $68 \%$ & $18 \%$ \\
\hline 7 & $3.0 \mathrm{~h}$ & $100 \%$ & $67 \%$ & $20 \%$ \\
\hline 8 & $4.0 \mathrm{~h}$ & $100 \%$ & $60 \%$ & $25 \%$ \\
\hline 9 & $5.0 \mathrm{~h}$ & $100 \%$ & $58 \%$ & $32 \%$ \\
\hline 10 & $6.0 \mathrm{~h}$ & $100 \%$ & $55 \%$ & $34 \%$ \\
\hline
\end{tabular}



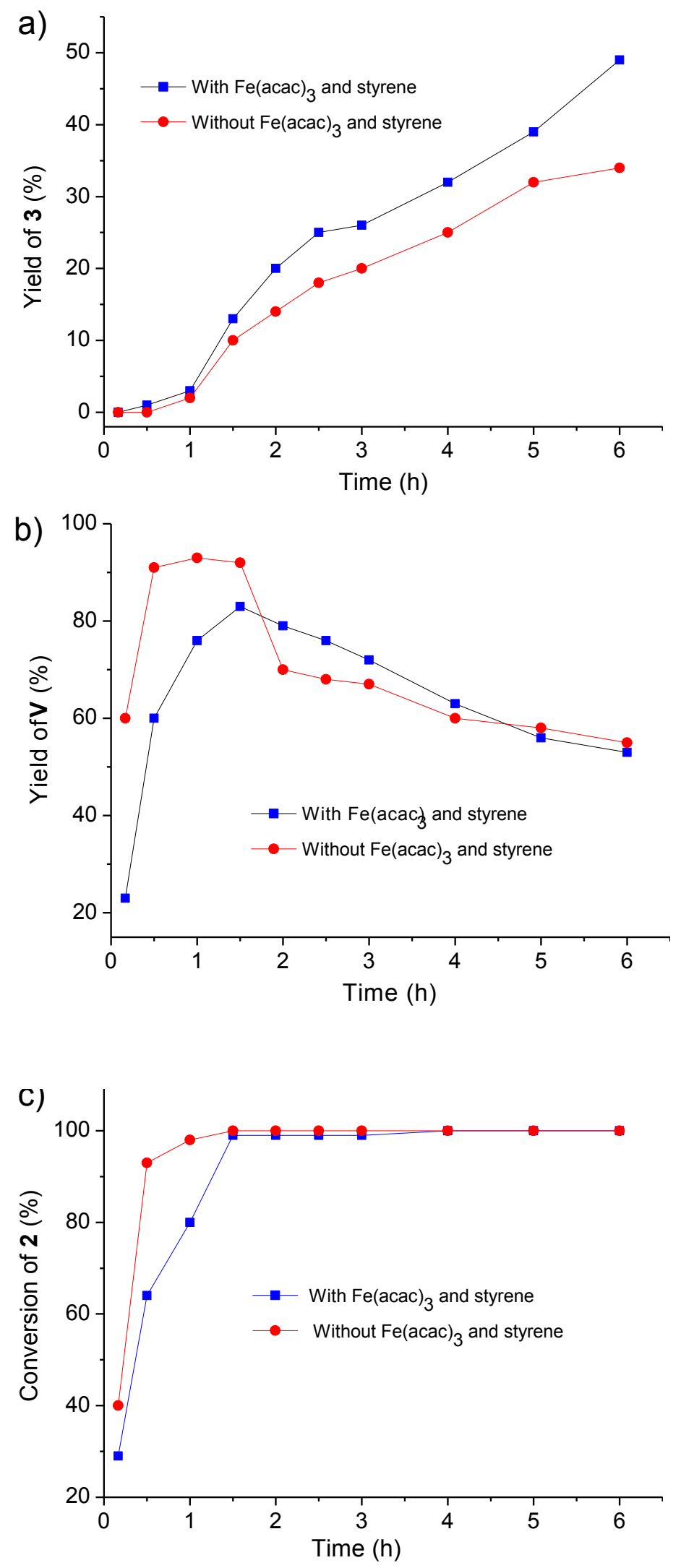

Figure S2. a) Yield of 3; b) Yield of V; c) Conversion of 2 
Procedure (with $\mathrm{Fe}(\mathbf{a c a c})_{3}$ and styrene): To a $25 \mathrm{~mL}$ of Schlenk tube were added anhydrous $\mathrm{K}_{2} \mathrm{CO}_{3}$ (powder, 4.0 equiv), and hydroquinone (2.0 equiv) in glove box, followed by $\mathrm{Fe}(\mathrm{acac})_{3}(3.5 \mathrm{~mol} \%)$, $\mathrm{PdCl}_{2}\left(\mathrm{PPh}_{3}\right)_{2}(5 \mathrm{~mol} \%)$, Xantphos $(7.5 \mathrm{~mol} \%)$, aryl boronic acid $1 \mathrm{a}(0.3 \mathrm{mmol})$, styrene (20 mol \%), ethyl bromodifluoroacetate 2 (2.0 equiv), and fresh distilled dioxane $(2.5 \mathrm{~mL})$. The reaction mixture was heated to $80{ }^{\circ} \mathrm{C}$ (oil bath). The reaction was monitored by ${ }^{19} \mathrm{~F}$ NMR using fluorobenzene as an internal standard.

Scheme S5. Cross-Coupling Reaction between Arylboronic Acid 1a and $\mathrm{BrCF}_{2} \mathrm{CO}_{2} \mathrm{~K} \mathrm{~V}$ with or without $\mathrm{Fe}(\text { acac })_{3}$ and Styrene.

With $\mathrm{Fe}(\mathrm{acac})_{3}$ and Styrene

a)

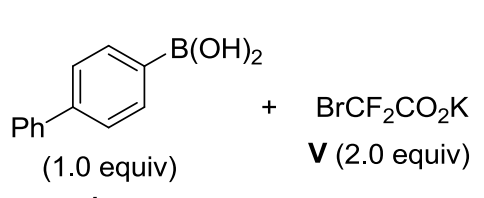

$1 \mathrm{a}$

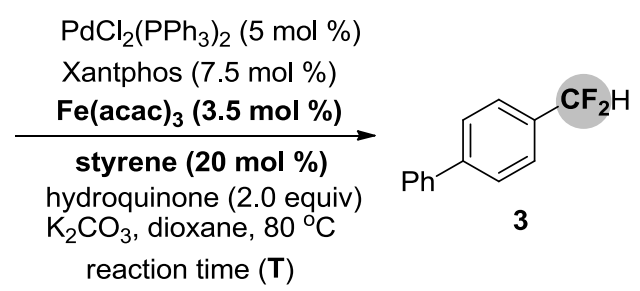

\begin{tabular}{|c|c|c|c|}
\hline Entry & Time & $\mathbf{V}$ (Recovery) & $\mathbf{3}$ (Yield) \\
\hline 1 & $10 \mathrm{~min}$ & $90 \%$ & $0 \%$ \\
\hline 2 & $30 \mathrm{~min}$ & $87 \%$ & $3 \%$ \\
\hline 3 & $1.0 \mathrm{~h}$ & $83 \%$ & $7 \%$ \\
\hline 4 & $1.5 \mathrm{~h}$ & $78 \%$ & $10 \%$ \\
\hline 5 & $2.0 \mathrm{~h}$ & $72 \%$ & $15 \%$ \\
\hline 6 & $2.5 \mathrm{~h}$ & $70 \%$ & $17 \%$ \\
\hline 7 & $3.0 \mathrm{~h}$ & $66 \%$ & $22 \%$ \\
\hline 8 & $4.0 \mathrm{~h}$ & $58 \%$ & $27 \%$ \\
\hline 9 & $5.0 \mathrm{~h}$ & $50 \%$ & $33 \%$ \\
\hline 10 & $6.0 \mathrm{~h}$ & $43 \%$ & $44 \%$ \\
\hline
\end{tabular}


Without $\mathrm{Fe}(\mathrm{acac})_{3}$ and Styrene

b)

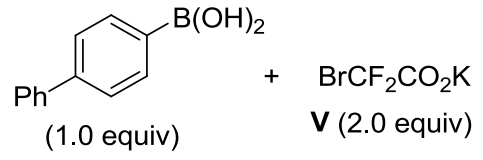

$1 \mathrm{a}$

$\mathrm{PdCl}_{2}\left(\mathrm{PPh}_{3}\right)_{2}(5 \mathrm{~mol} \%)$

Xantphos $(7.5 \mathrm{~mol} \%)$

hydroquinone (2.0 equiv) $\mathrm{K}_{2} \mathrm{CO}_{3}$, dioxane, $80^{\circ} \mathrm{C}$

reaction time $(\mathbf{T})$

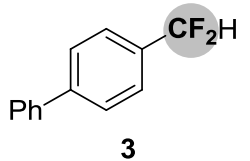

\begin{tabular}{|c|c|c|c|}
\hline Entry & Time & V(Recovery) & $\mathbf{3}$ (Yield) \\
\hline 1 & $10 \mathrm{~min}$ & $100 \%$ & $0 \%$ \\
\hline 2 & $30 \mathrm{~min}$ & $100 \%$ & $0 \%$ \\
\hline 3 & $1.0 \mathrm{~h}$ & $95 \%$ & $1 \%$ \\
\hline 4 & $1.5 \mathrm{~h}$ & $82 \%$ & $2 \%$ \\
\hline 5 & $2.0 \mathrm{~h}$ & $76 \%$ & $7 \%$ \\
\hline 6 & $2.5 \mathrm{~h}$ & $73 \%$ & $8 \%$ \\
\hline 7 & $3.0 \mathrm{~h}$ & $70 \%$ & $10 \%$ \\
\hline 8 & $4.0 \mathrm{~h}$ & $66 \%$ & $15 \%$ \\
\hline 9 & $5.0 \mathrm{~h}$ & $60 \%$ & $19 \%$ \\
\hline 10 & $6.0 \mathrm{~h}$ & $50 \%$ & $27 \%$ \\
\hline
\end{tabular}

a)

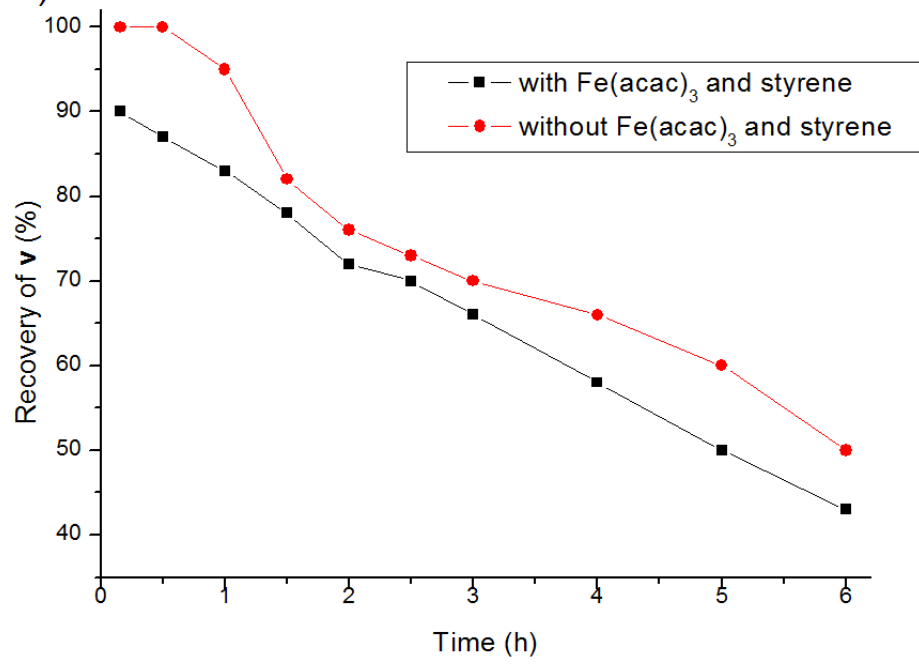




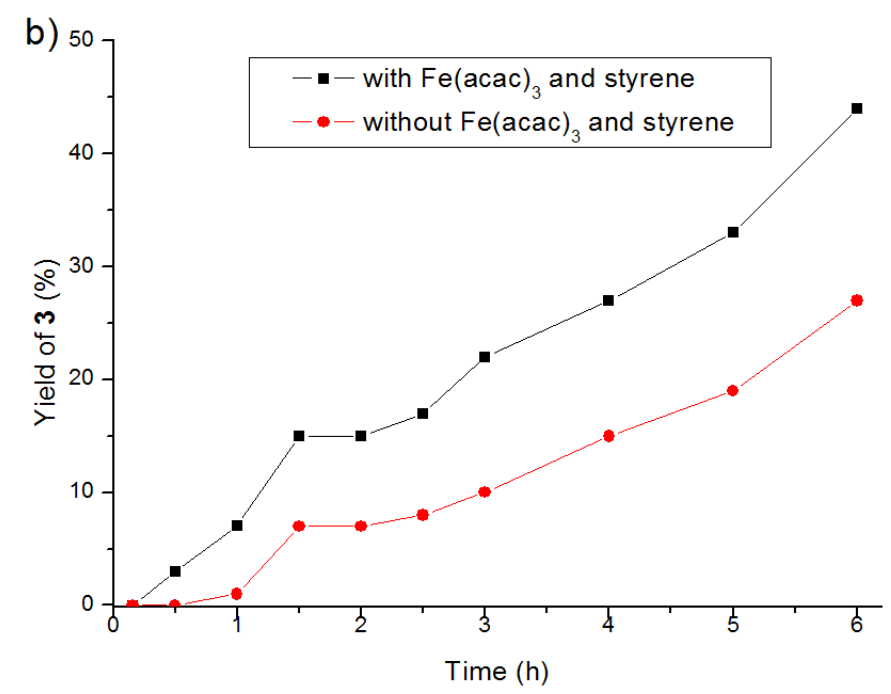

Figure S3. a) Recovery of V; b) Yield of 3.

\section{Identification of Difluorocarbene}

Scheme S6. Reaction of 1a with V under Standard Reaction Conditions in the Presence of Pyridine-2-thiol
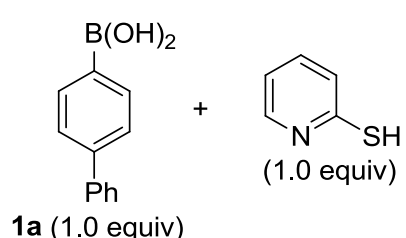

$$
\mathrm{Pd}\left(\mathrm{PPh}_{3}\right) \mathrm{Cl}_{2}(5 \mathrm{~mol} \%)
$$

Xantphos (7.5 mol\%)

$\frac{\mathrm{BrCF}_{2} \mathrm{CO}_{2} \mathrm{~K} \text { (2.0 equiv) }}{\text { hydroquinone (2.0 equiv) }}$

$\mathrm{K}_{2} \mathrm{CO}_{3}$, dioxane, $80^{\circ} \mathrm{C}$

3, ND +

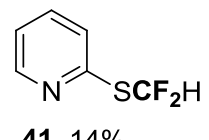

$41,14 \%$

1a (1.0 equiv)

Procedure: To a $25 \mathrm{~mL}$ of Schlenk tube were added anhydrous $\mathrm{K}_{2} \mathrm{CO}_{3}$ (powder, 4.0 equiv), hydroquinone (2.0 equiv), 2-mercaptopyridine, $\mathrm{BrCF}_{2} \mathrm{CO}_{2} \mathrm{~K} \mathrm{~V}(0.6 \mathrm{mmol}), \mathrm{PdCl}_{2}\left(\mathrm{PPh}_{3}\right)_{2}(5 \mathrm{~mol} \%)$, Xantphos $(7.5 \mathrm{~mol} \%)$, aryl boronic acid $1 \mathrm{a}(0.3 \mathrm{mmol})$, and fresh distilled dioxane $(2.5 \mathrm{~mL})$ under $\mathrm{N}_{2}$. The reaction mixture was heated to $80{ }^{\circ} \mathrm{C}$ (oil bath) for $24 \mathrm{~h}$ and provided 41 in $14 \%$ yield (determined by ${ }^{19} \mathrm{~F}$ NMR using fluorobenzene as an internal standard). Compound 41: This compound is known. ${ }^{1}$ ${ }^{19} \mathrm{~F}$ NMR $\left(376 \mathrm{MHz}, \mathrm{CDCl}_{3}\right) \delta-97.3(\mathrm{~d}, J=56.1 \mathrm{~Hz}, 2 \mathrm{~F}) . \mathrm{MS}(\mathrm{EI}): m / z(\%) 161\left(\mathrm{M}^{+}\right)$. 


\section{Scheme S7. Reaction of 1a with V in the Presence of Palladium Complex VI.}

a)<smiles>COc1ccc(-c2ccccc2)cc1</smiles>

b)

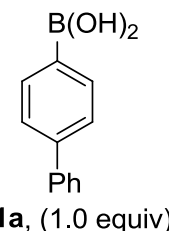<smiles>CC1(C)C=C2C=CC=C1c1c(Oc3ccccc3)cccc1P(c1ccccc1)c1ccccc1P2(I)(I)Pc1ccccc1</smiles>

VI, (20 mol\%)<smiles>CC1(C)c2cccc(P(I)c3ccccc3)c2Oc2c1cccc2P(I)(I)(c1ccccc1)c1ccccc1</smiles>

VI, $(50 \mathrm{~mol} \%)$

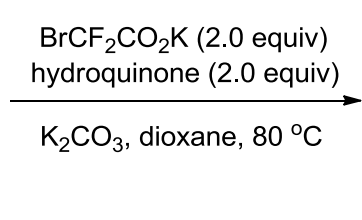

$\mathrm{BrCF}_{2} \mathrm{CO}_{2} \mathrm{~K}(2.0$ equiv) hydroquinone ( 2.0 equiv)

$\mathrm{K}_{2} \mathrm{CO}_{3}$, dioxane, $80^{\circ} \mathrm{C}$

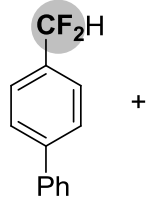

$3,47 \%$

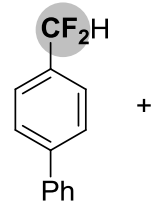

$3,16 \%$<smiles>FC(F)(F)C(F)(F)c1ccccc1</smiles>

$3 a, 20 \%$

ND<smiles>O=[N+]([O-])c1cccc(C(F)(F)F)c1</smiles>

3a, $30 \%$

ND

Procedure: To a $25 \mathrm{~mL}$ of Schlenk tube were added anhydrous $\mathrm{K}_{2} \mathrm{CO}_{3}$ (powder, 4.0 equiv), hydroquinone (2.0 equiv), $\mathrm{BrCF}_{2} \mathrm{CO}_{2} \mathrm{~K} \mathrm{~V}$ (0.6 mmol), [PhPd(Xantphos)I] ${ }^{2}$ (20 mol\% or $50 \mathrm{~mol} \%$ ), aryl boronic acid 1a $(0.3 \mathrm{mmol})$, and fresh distilled dioxane $(2.5 \mathrm{~mL})$ under $\mathrm{N}_{2}$. The reaction mixture was heated to $80^{\circ} \mathrm{C}$ (oil bath) for $24 \mathrm{~h}$ and provided compound 3 and 4-(1,1,2,2-tetrafluoroethyl)-1,1'biphenyl 3a (the yields were determined by ${ }^{19} \mathrm{~F}$ NMR using fluorobenzene as an internal standard). 4-(1,1,2,2-tetrafluoroethyl)-1,1'-biphenyl (3a). This compound is known. ${ }^{3}{ }^{1} \mathrm{H}$ NMR (400 MHz, $\left.\mathrm{CDCl}_{3}\right) \delta 7.12-7.69(\mathrm{~m}, 2 \mathrm{H}), 7.64-7.59(\mathrm{~m}, 4 \mathrm{H}), 7.49-7.45(\mathrm{~m}, 2 \mathrm{H}), 7.43-7.38(\mathrm{~m}, 1 \mathrm{H}), 5.96(\mathrm{tt}, J=$ $54.4 \mathrm{~Hz}, J=2.4 \mathrm{~Hz}, 1 \mathrm{H}) .{ }^{19} \mathrm{~F}$ NMR $\left(376 \mathrm{MHz}, \mathrm{CDCl}_{3}\right) \delta-113.4(\mathrm{~m}, 2 \mathrm{~F}),-134.1(\mathrm{dt}, J=54.4 \mathrm{~Hz}, J$ $=3.8 \mathrm{~Hz}, 2 \mathrm{~F}) . \mathrm{MS}(\mathrm{EI}): \mathrm{m} / z(\%) 254\left(\mathrm{M}^{+}\right), 203(100)$. HRMS calcd. for $\mathrm{C}_{14} \mathrm{H}_{10} \mathrm{~F}_{4}\left(\mathrm{M}^{+}\right): 254.0719$; Found: 254.0718 .

Scheme S8. Cross-Coupling Reaction between $1 \mathrm{a}$ and 2 in the Presence of $\mathrm{Pd}(\mathrm{PPh} 3)_{4}$ with or without $\mathrm{Fe}(\mathrm{acac}) 3$.

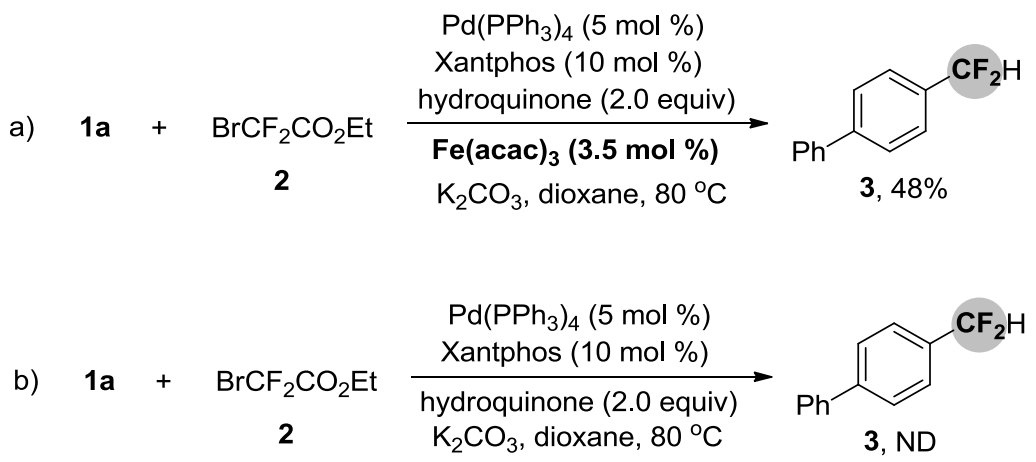


Procedure (with Fe(acac)3): To a $25 \mathrm{~mL}$ of Schlenk tube were added anhydrous $\mathrm{K}_{2} \mathrm{CO}_{3}$ (powder, 4.0

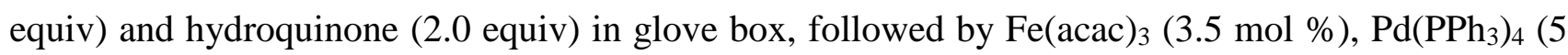
mol \%), Xantphos (10 mol \%), aryl boronic acid $\mathbf{1 a}(0.3 \mathrm{mmol})$, ethyl bromodifluoroacetate 2 (2.0 equiv), and fresh distilled dioxane $(2.0 \mathrm{~mL})$. The reaction mixture was heated to $80{ }^{\circ} \mathrm{C}$ (oil bath). The reaction was monitored by ${ }^{19} \mathrm{~F}$ NMR using fluorobenzene as an internal standard.

\section{X-Ray Photoelectron Spectroscopy (XPS) Analysis of the Reaction}

Scheme S9a. X-Ray Photoelectron Spectroscopy (XPS) Analysis of Reaction of 1a with 2 under Standard Reaction Conditions

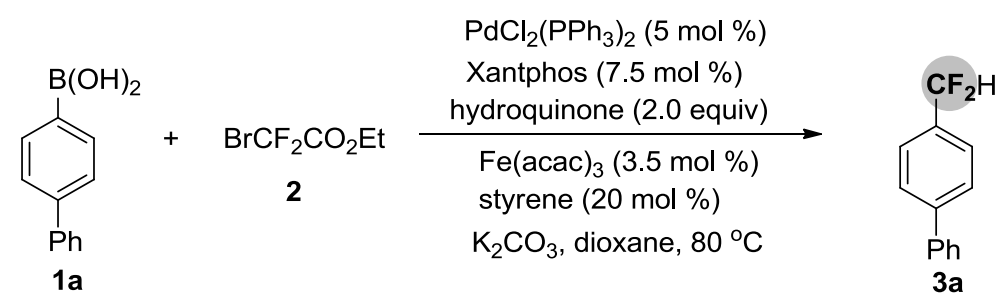

Procedure: To a $25 \mathrm{~mL}$ of Schlenk tube were added anhydrous $\mathrm{K}_{2} \mathrm{CO}_{3}$ (powder, 4.0 equiv), and hydroquinone (2.0 equiv) in glove box, followed by $\mathrm{Fe}(\mathrm{acac})_{3}(3.5 \mathrm{~mol} \%), \mathrm{PdCl}_{2}\left(\mathrm{PPh}_{3}\right)_{2}(5 \mathrm{~mol} \%)$, Xantphos (7.5 mol \%), aryl boronic acid 1a $(0.3 \mathrm{mmol})$, styrene (20 mol \%), ethyl bromodifluoroacetate 2 (2.0 equiv), and fresh distilled dioxane $(2.5 \mathrm{~mL})$. The reaction mixture was heated to $80{ }^{\circ} \mathrm{C}$ (oil bath). After stirring for $24 \mathrm{~h}$, the reaction was cooled to room temperature and concentrated under $\mathrm{N}_{2}$. The resulting mixture was analyzed by X-ray photoelectron spectroscopy (XPS) (Note: all the experiments were carried out under $\mathrm{N}_{2}$ ). The XPS showed that peak corresponding to $\mathrm{Pd}^{\mathrm{II}} 3 \mathrm{~d}_{5 / 2}$ was observed with the binding energy at $336.65 \mathrm{ev}$, which was negatively shifted by $0.85 \mathrm{ev}$ compared with free $\mathrm{Pd}\left(\mathrm{PPh}_{3}\right)_{2} \mathrm{Cl}_{2}\left(\mathrm{Pd}^{\mathrm{II}} 3 \mathrm{~d}_{5 / 2}\right.$ at $\left.337.5 \mathrm{ev}\right){ }^{4}$
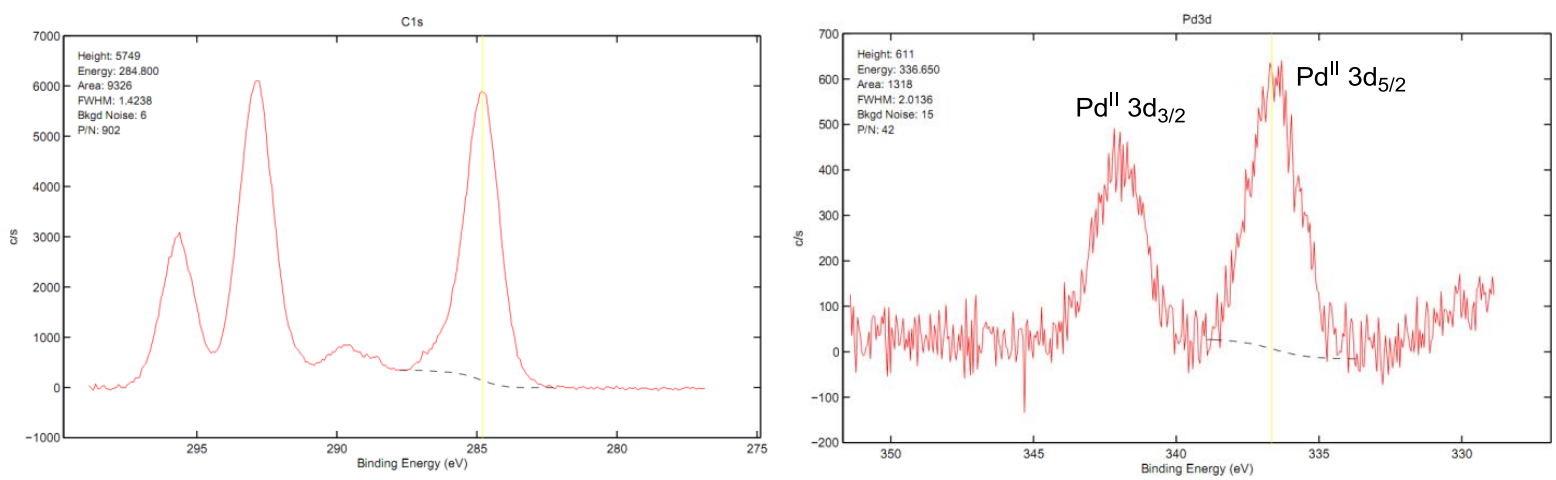

Figure S4a. XPS Analysis of Reaction of 1a with 2 under Standard Reaction Conditions 
Scheme S9b. X-Ray Photoelectron Spectroscopy (XPS) Analysis of Reaction of 1a with 2 in the Absence of $\mathrm{Fe}(\mathrm{acac})_{3}$ and Styrene.

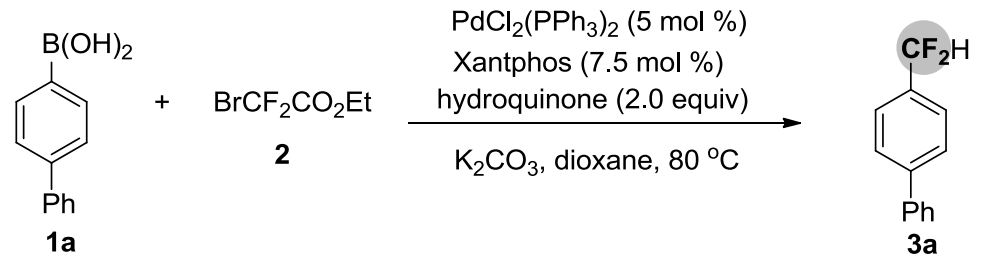

Procedure: To a $25 \mathrm{~mL}$ of Schlenk tube were added anhydrous $\mathrm{K}_{2} \mathrm{CO}_{3}$ (powder, 4.0 equiv), and hydroquinone (2.0 equiv) in glove box, followed by $\mathrm{PdCl}_{2}\left(\mathrm{PPh}_{3}\right)_{2}(5 \mathrm{~mol} \%)$, Xantphos (7.5 mol \%), aryl boronic acid $\mathbf{1 a}(0.3 \mathrm{mmol})$, ethyl bromodifluoroacetate $\mathbf{2}$ (2.0 equiv), and fresh distilled dioxane $\left(2.5 \mathrm{~mL}\right.$ ). The reaction mixture was heated to $80^{\circ} \mathrm{C}$ (oil bath). After stirring for $24 \mathrm{~h}$, the reaction was cooled to room temperature and concentrated under $\mathrm{N}_{2}$. The resulting mixture was analyzed by X-ray photoelectron spectroscopy (XPS) (Note: all the experiments were carried out under $\mathrm{N}_{2}$ ). The XPS showed that peak corresponding to $\mathrm{Pd}^{\mathrm{II}} 3 \mathrm{~d}_{5 / 2}$ was observed with the binding energy at $336.85 \mathrm{ev}$, which was negatively shifted by 0.65 ev compared with free $\mathrm{Pd}\left(\mathrm{PPh}_{3}\right)_{2} \mathrm{Cl}_{2}\left(\mathrm{Pd}^{\mathrm{II}} 3 \mathrm{~d}_{5 / 2}\right.$ at $\left.337.5 \mathrm{ev}\right){ }^{4}$
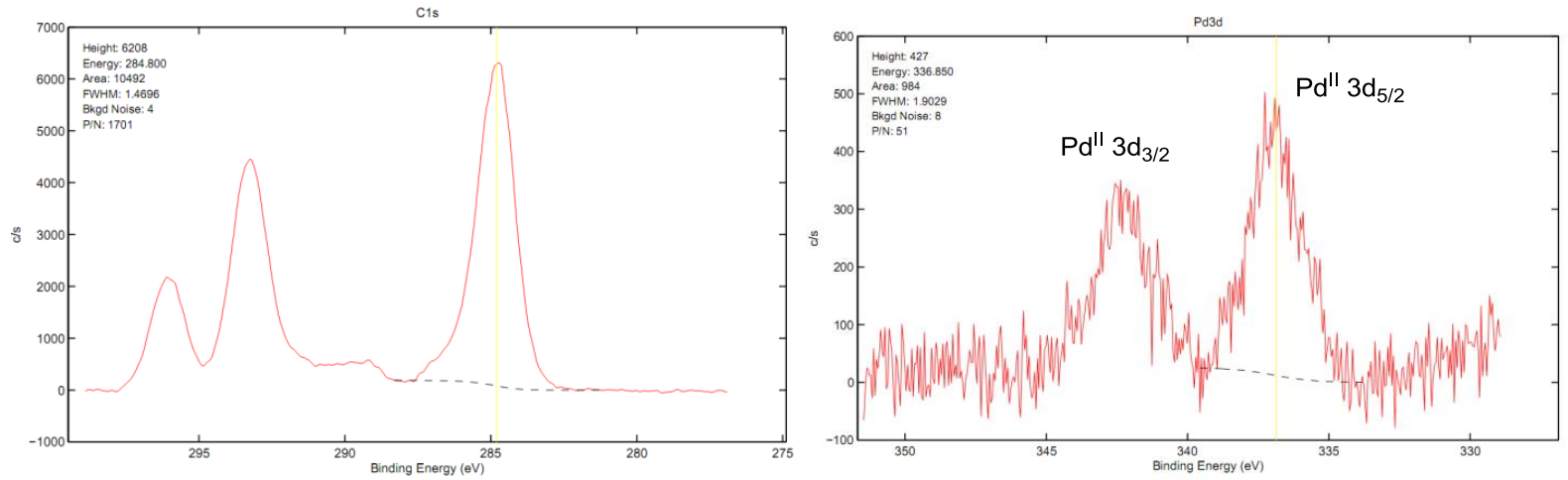

Figure S4b. XPS Analysis of Reaction of $1 \mathbf{a}$ with $\mathbf{2}$ in the Absence of Fe(acac) 3 and Styrene

Scheme S10c. X-Ray Photoelectron Spectroscopy (XPS) Analysis of Reaction of 1a with 2 in the Presence of $\mathrm{Pd}\left(\mathrm{PPh}_{3}\right)_{4}$ and $\mathrm{Fe}(\mathrm{acac})_{3}$.

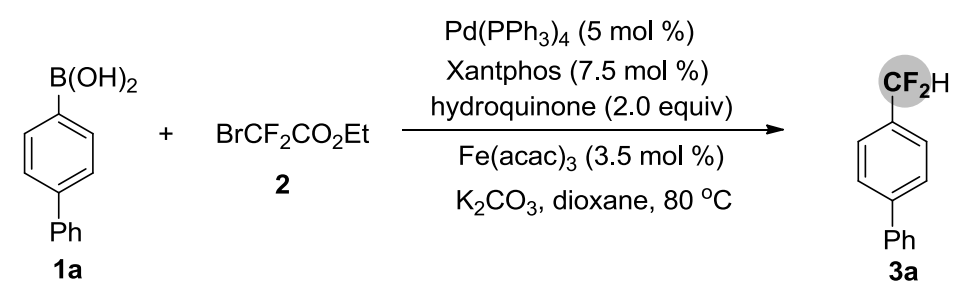

Procedure: To a $25 \mathrm{~mL}$ of Schlenk tube were added anhydrous $\mathrm{K}_{2} \mathrm{CO}_{3}$ (powder, 4.0 equiv), and hydroquinone (2.0 equiv) in glove box, followed by $\mathrm{Fe}(\mathrm{acac})_{3}(3.5 \mathrm{~mol} \%), \mathrm{Pd}\left(\mathrm{PPh}_{3}\right)_{4}(5 \mathrm{~mol} \%)$, Xantphos (7.5 mol \%), aryl boronic acid 1a $(0.3 \mathrm{mmol})$, ethyl bromodifluoroacetate 2 (2.0 equiv), and fresh distilled dioxane $(2.5 \mathrm{~mL})$. The reaction mixture was heated to $80^{\circ} \mathrm{C}$ (oil bath). After stirring for $24 \mathrm{~h}$, the reaction was cooled to room temperature. After stirring for $24 \mathrm{~h}$, the reaction was cooled to 
room temperature and concentrated under $\mathrm{N}_{2}$. The resulting mixture was analyzed by X-ray photoelectron spectroscopy (XPS) (Note: all the experiments were carried out under $\mathrm{N}_{2}$ ). The XPS showed that peak corresponding to $\mathrm{Pd}^{\mathrm{II}} 3 \mathrm{~d}_{5 / 2}$ was observed with the binding energy at $336.80 \mathrm{ev}$, which was negatively shifted by $0.70 \mathrm{ev}$ compared with free $\mathrm{Pd}\left(\mathrm{PPh}_{3}\right)_{2} \mathrm{Cl}_{2}\left(\mathrm{Pd}^{\mathrm{II}} 3 \mathrm{~d}_{5 / 2}\right.$ at $\left.337.5 \mathrm{ev}\right){ }^{4}$
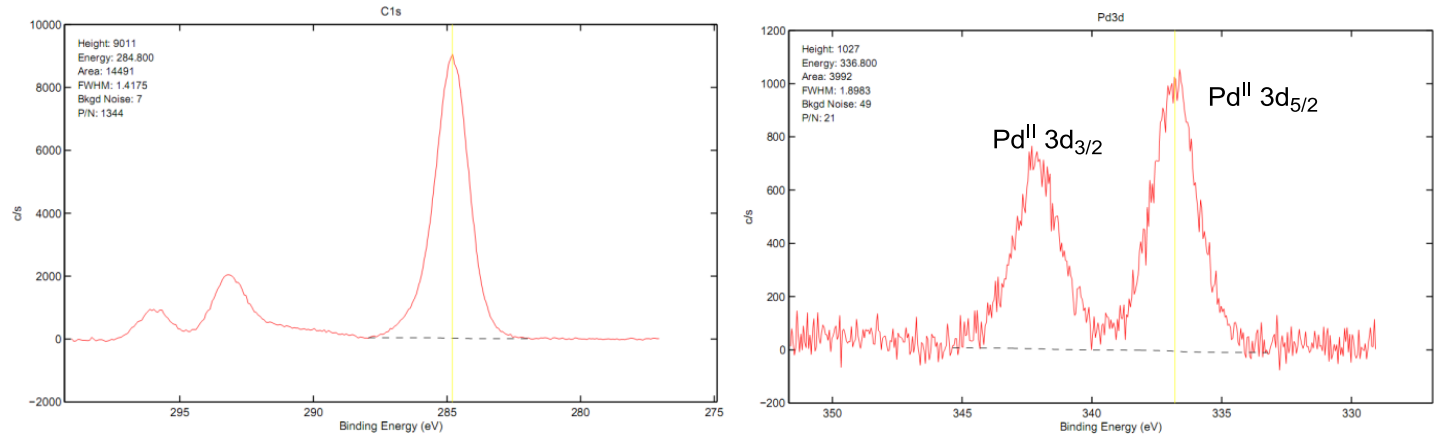

Figure S4c. XPS Analysis of Reaction of $1 a$ with 2 in the Presence of $\mathrm{Pd}\left(\mathrm{PPh}_{3}\right)_{4}$ and $\mathrm{Fe}(\mathrm{acac})_{3}$

\section{General Procedure for Pd-Catalyzed Difluoromethylation of Arylboronic Acids with Bromodifluoroacetate}

To a $25 \mathrm{~mL}$ of Schlenk tube were added anhydrous $\mathrm{K}_{2} \mathrm{CO}_{3}$ (powder, 4.0 equiv), and hydroquinone (2.0 equiv) in glove box, followed by $\mathrm{Fe}(\mathrm{acac})_{3}(3.5 \mathrm{~mol} \%), \mathrm{PdCl}_{2}\left(\mathrm{PPh}_{3}\right)_{2}(5 \mathrm{~mol} \%)$, Xantphos $(7.5 \mathrm{~mol} \%)$, aryl boronic acid $1(0.3 \mathrm{mmol})$, styrene (20 mol \%), ethyl bromodifluoroacetate 2 (2.0 equiv), and fresh distilled dioxane $(2.5 \mathrm{~mL})$. The reaction mixture was heated to $80{ }^{\circ} \mathrm{C}$ (oil bath). After stirring for $24 \mathrm{~h}$, the reaction was cooled to room temperature. The reaction mixture was diluted with EtOAc and filtered with a pad of cellite. The filtrate was concentrated, and the residue was purified with silica gel chromatography to give product.<smiles>FC(F)(F)c1ccc(-c2ccccc2)cc1</smiles>

4-(Difluoromethyl)-1,1'-biphenyl (3). The product (52 mg, 85\% yield) as a white solid (m.p. 78-82 $\left.{ }^{\circ} \mathrm{C}\right)$ was purified with silica gel chromatography (Petroleum ether). This compound is known. ${ }^{5}{ }^{1} \mathrm{H}$ NMR (300 MHz, $\left.\mathrm{CDCl}_{3}\right)$ 8 7.69-7.67 (m, $\left.2 \mathrm{H}\right)$, 7.62-7.58 (m, 4 H), 7.49-7.45 (m, 2 H), 7.41-7.37 (m, $1 \mathrm{H}), 6.71(\mathrm{t}, J=56.4 \mathrm{~Hz}, 1 \mathrm{H}) .{ }^{19} \mathrm{~F}$ NMR $\left(282 \mathrm{MHz}, \mathrm{CDCl}_{3}\right) \delta-110.7(\mathrm{~d}, J=56.4 \mathrm{~Hz}, 2 \mathrm{~F}) .{ }^{13} \mathrm{C}$ NMR $\left(125.7 \mathrm{MHz}, \mathrm{CDCl}_{3}\right) \delta 143.7(\mathrm{t}, J=2.0 \mathrm{~Hz}), 140.2,133.2(\mathrm{t}, J=22.4 \mathrm{~Hz}), 128.9,127.9,127.4,127.2$, $126.0(\mathrm{t}, J=6.0 \mathrm{~Hz}), 114.7(\mathrm{t}, J=238.3 \mathrm{~Hz}) . \mathrm{MS}(\mathrm{EI}): \mathrm{m} / z(\%) 204\left(\mathrm{M}^{+}\right), 204(100)$. HRMS calcd. for 
$\mathrm{C}_{13} \mathrm{H}_{10} \mathrm{~F}_{2}\left(\mathrm{M}^{+}\right): 204.0751$; Found: 204.0753.<smiles>FC(F)(F)c1ccccc1-c1ccccc1</smiles>

2-(Difluoromethyl)-1,1'-biphenyl (5). The product (39 mg, 64\% yield) as a colorless oil was purified with silica gel chromatography (Petroleum ether). ${ }^{1} \mathrm{H} \mathrm{NMR}\left(400 \mathrm{MHz}, \mathrm{CDCl}_{3}\right) \delta 7.79(\mathrm{dd}, J=6.8 \mathrm{~Hz}$, $J=2.0 \mathrm{~Hz}, 1 \mathrm{H}), 7.54-7.48(\mathrm{~m}, 2 \mathrm{H}), 7.47-7.41(\mathrm{~m}, 3 \mathrm{H}), 7.36-7.34(\mathrm{~m}, 3 \mathrm{H}), 6.54$ (t, $J=54.8 \mathrm{~Hz}, 1$ H). ${ }^{19} \mathrm{~F}$ NMR $\left(376 \mathrm{MHz}, \mathrm{CDCl}_{3}\right) \delta-107.4(\mathrm{~d}, J=54.8 \mathrm{~Hz}, 2 \mathrm{~F}) .{ }^{13} \mathrm{C}$ NMR $\left(100 \mathrm{MHz}, \mathrm{CDCl}_{3}\right) \delta 141.4$ $(\mathrm{t}, J=6.6 \mathrm{~Hz}), 138.6,131.7(\mathrm{t}, J=22.2 \mathrm{~Hz}), 130.5(\mathrm{t}, J=1.8 \mathrm{~Hz}), 130.2,129.4,128.4,127.9,127.8$, $125.6(\mathrm{t}, J=5.2 \mathrm{~Hz}), 113.1(\mathrm{t}, J=234.6 \mathrm{~Hz}) . \mathrm{MS}(\mathrm{EI}): \mathrm{m} / z(\%) 204\left(\mathrm{M}^{+}\right), 183,154,58(100) . \mathrm{HRMS}$ calcd. for $\mathrm{C}_{13} \mathrm{H}_{10} \mathrm{~F}_{2}\left(\mathrm{M}^{+}\right)$: 204.0751; Found: 204.0753.<smiles>FC(F)(F)c1cccc(-c2ccccc2)c1</smiles>

3-(Difluoromethyl)-1,1'-biphenyl (6). The product (54 mg, 89\% yield) as a colorless oil was purified with silica gel chromatography (Petroleum ether). ${ }^{1} \mathrm{H}$ NMR (400 MHz, $\left.\mathrm{CDCl}_{3}\right) \delta 7.75$ (s, $\left.1 \mathrm{H}\right), 7.73$ $7.71(\mathrm{~m}, 1 \mathrm{H}), 7.63-7.62(\mathrm{~m}, 1 \mathrm{H}), 7.61-7.60(\mathrm{~m}, 1 \mathrm{H}), 7.55(\mathrm{t}, J=7.2 \mathrm{~Hz}, 1 \mathrm{H})$, 7.52-7.46 (m, $3 \mathrm{H})$, $7.40(\mathrm{tt}, J=7.2 \mathrm{~Hz}, 1 \mathrm{H}), J=1.2 \mathrm{~Hz}, 1 \mathrm{H}), 6.72(\mathrm{t}, J=56.4 \mathrm{~Hz}, 1 \mathrm{H}) .{ }^{19} \mathrm{~F} \mathrm{NMR}\left(376 \mathrm{MHz}, \mathrm{CDCl}_{3}\right) \delta$ $-110.6(\mathrm{~d}, J=56.4 \mathrm{~Hz}, 2 \mathrm{~F}) .{ }^{13} \mathrm{C}$ NMR $\left(100 \mathrm{MHz}, \mathrm{CDCl}_{3}\right) \delta 141.9,140.2,134.9(\mathrm{t}, J=22.0 \mathrm{~Hz}), 129.5$ $(\mathrm{t}, J=1.9 \mathrm{~Hz}), 129.2,128.9,127.8,127.2,124.4(\mathrm{t}, J=5.9 \mathrm{~Hz}), 124.3(\mathrm{t}, J=5.9 \mathrm{~Hz}), 114.8(\mathrm{t}, J=237.6$ Hz). MS (EI): $m / z(\%) 204\left(\mathrm{M}^{+}\right), 204$ (100). HRMS calcd. for $\mathrm{C}_{13} \mathrm{H}_{10} \mathrm{~F}_{2}\left(\mathrm{M}^{+}\right)$: 204.0751; Found: 204.0754 .<smiles>CC(C)(C)c1ccc(C(F)(F)F)cc1</smiles>

1-(Tert-butyl)-4-(difluoromethyl)benzene (7). The product (33 $\mathrm{mg}, 60 \%$ yield) as a colorless oil was purified with silica gel chromatography (Petroleum ether). This compound is known. ${ }^{5} \mathrm{H}$ NMR (400 $\left.\mathrm{MHz}, \mathrm{CDCl}_{3}\right) \delta 7.49(\mathrm{~d}, J=8.8 \mathrm{~Hz}, 2 \mathrm{H}), 7.45(\mathrm{~d}, J=8.8 \mathrm{~Hz}, 2 \mathrm{H}), 6.64(\mathrm{t}, J=56.8 \mathrm{~Hz}, 1 \mathrm{H}), 1.35(\mathrm{~s}$, 
$9 \mathrm{H}) .{ }^{19} \mathrm{~F}$ NMR $\left(375 \mathrm{MHz}, \mathrm{CDCl}_{3}\right) \delta-109.9(\mathrm{~d}, J=56.8 \mathrm{~Hz}, 2 \mathrm{~F}) .{ }^{13} \mathrm{C} \mathrm{NMR}\left(125.7 \mathrm{MHz}, \mathrm{CDCl}_{3}\right) \delta$ $153.9(\mathrm{t}, J=2.0 \mathrm{~Hz}), 131.6(\mathrm{t}, J=22.4 \mathrm{~Hz}), 125.6,125.3(\mathrm{t}, J=5.8 \mathrm{~Hz}), 114.9(\mathrm{t}, J=237.9 \mathrm{~Hz}), 34.8$, 31.2. MS (EI): $m / z(\%) 184\left(\mathrm{M}^{+}\right), 169$ (100). HRMS calcd. for $\mathrm{C}_{11} \mathrm{H}_{14} \mathrm{~F}_{2}\left(\mathrm{M}^{+}\right)$: 184.1064 ; Found:184.1063.<smiles>FC(F)(F)c1ccc(OCc2ccccc2)cc1</smiles>

1-(Benzyloxy)-4-(difluoromethyl)benzene (8). The product (47 mg, 67\% yield) as a wihte solid (m.p. 78-80 ${ }^{\circ} \mathrm{C}$ ) was purified with silica gel chromatography (Petroleum ether/EtOAc $=40: 1$ ). This compound is known. ${ }^{51} \mathrm{H}$ NMR (400 MHz, $\left.\mathrm{CDCl}_{3}\right) \delta$ 7.45-7.43 (m, $\left.4 \mathrm{H}\right)$, 7.42-7.38 (m, $\left.2 \mathrm{H}\right)$, 7.36-7.32 $(\mathrm{m}, 1 \mathrm{H}), 7.03(\mathrm{~d}, J=8.8 \mathrm{~Hz}, 2 \mathrm{H}), 6.61(\mathrm{t}, J=56.8 \mathrm{~Hz}, 1 \mathrm{H}), 5.11(\mathrm{~s}, 2 \mathrm{H}) .{ }^{19} \mathrm{~F}$ NMR $(376 \mathrm{MHz}$, $\left.\mathrm{CDCl}_{3}\right) \delta-108.3(\mathrm{~d}, J=56.8 \mathrm{~Hz}, 2 \mathrm{~F}) .{ }^{13} \mathrm{C} \mathrm{NMR}\left(100 \mathrm{MHz}, \mathrm{CDCl}_{3}\right) \delta 160.5(\mathrm{t}, J=1.4 \mathrm{~Hz}), 136.5$, 128.7, 128.2, 127.5, $127.2(\mathrm{t}, J=6.0 \mathrm{~Hz}), 127.0,114.9,114.8(\mathrm{t}, J=236.0 \mathrm{~Hz}), 70.1 . \mathrm{MS}(\mathrm{EI}): \mathrm{m} / z(\%)$ $234\left(\mathrm{M}^{+}\right), 91(100)$. HRMS calcd. for $\mathrm{C}_{14} \mathrm{H}_{12} \mathrm{OF}_{2}\left(\mathrm{M}^{+}\right)$: 234.0856; Found: 234.0859. IR (thin film) $v_{\max }$ $3032,2940,1615,1598 \mathrm{~cm}^{-1}$.<smiles>FC(F)(F)c1ccc(Oc2ccccc2)cc1</smiles>

1-(Difluoromethyl)-4-phenoxybenzene (9). The product (41 $\mathrm{mg}, 62 \%$ yield) as a colorless oil was purified with silica gel chromatography (Petroleum ether/EtOAc $=40: 1) .{ }^{1} \mathrm{H} \mathrm{NMR}\left(400 \mathrm{MHz}, \mathrm{CDCl}_{3}\right)$ $\delta 7.47(\mathrm{~d}, J=8.4 \mathrm{~Hz}, 2 \mathrm{H}), 7.39-7.35(\mathrm{~m}, 2 \mathrm{H}), 7.17$ (tt, $J=7.2 \mathrm{~Hz}, J=1.2 \mathrm{~Hz}, 1 \mathrm{H}), 7.06$ (m, $2 \mathrm{H})$, $7.03(\mathrm{~s}, 2 \mathrm{H}), 6.63(\mathrm{t}, J=56.4 \mathrm{~Hz}, 1 \mathrm{H}) .{ }^{19} \mathrm{~F} \mathrm{NMR}\left(376 \mathrm{MHz}, \mathrm{CDCl}_{3}\right) \delta-109.1(\mathrm{~d}, J=56.4 \mathrm{~Hz}, 2 \mathrm{~F})$. ${ }^{13} \mathrm{C} \mathrm{NMR}\left(100 \mathrm{MHz}, \mathrm{CDCl}_{3}\right) \delta 159.6(\mathrm{t}, J=2.0 \mathrm{~Hz}), 156.2,129.9,128.9(\mathrm{t}, J=22.6 \mathrm{~Hz}), 127.3(\mathrm{t}, J=$ 6.0 Hz), 124.1, 119.6, 118.3, 114.6 (t, $J=236.8 \mathrm{~Hz})$. MS (EI): $m / z(\%) 220\left(\mathrm{M}^{+}\right), 220$ (100). HRMS calcd. for $\mathrm{C}_{13} \mathrm{H}_{10} \mathrm{OF}_{2}\left(\mathrm{M}^{+}\right)$: 220.0700; Found: 220.0698. IR (thin film) $v_{\max } 3041,2963,1765,1590$ $\mathrm{cm}^{-1}$. 
$\underbrace{C F_{2} \mathrm{H}}_{\mathrm{OMe}}$

1-(Difluoromethyl)-4-methoxybenzene (10). The product (33 $\mathrm{mg}, 70 \%$ yield) as a colorless oil was purified with silica gel chromatography (Petroleum ether/EtOAc $=30: 1$ ). This compound is known. ${ }^{6}$ ${ }^{1} \mathrm{H}$ NMR (400 MHz, $\left.\mathrm{CDCl}_{3}\right) \delta 7.44(\mathrm{~d}, J=8.4 \mathrm{~Hz}, 2 \mathrm{H}), 6.95(\mathrm{~d}, J=8.4 \mathrm{~Hz}, 2 \mathrm{H}), 6.60(\mathrm{t}, J=56.8 \mathrm{~Hz}$, $1 \mathrm{H}), 3.84$ (s, $3 \mathrm{H}) .{ }^{19} \mathrm{~F}$ NMR $\left(376 \mathrm{MHz}, \mathrm{CDCl}_{3}\right) \delta-108.3$ (d, J=56.4 Hz, $\left.2 \mathrm{~F}\right) .{ }^{13} \mathrm{C} \mathrm{NMR}(125.7 \mathrm{MHz}$, $\left.\mathrm{CDCl}_{3}\right) \delta 161.4,127.1(\mathrm{t}, J=6.0 \mathrm{~Hz}), 126.8(\mathrm{t}, J=22.8 \mathrm{~Hz}), 114.9(\mathrm{t}, J=237.3 \mathrm{~Hz}), 113.9,55.3 . \mathrm{MS}$ (EI): $m / z(\%) 158\left(\mathrm{M}^{+}\right), 158$ (100). HRMS calcd. for $\mathrm{C}_{8} \mathrm{H}_{8} \mathrm{OF}_{2}\left(\mathrm{M}^{+}\right)$: 158.0543; Found: 158.0541 .<smiles>COc1cc(OC)cc(C(F)(F)F)c1</smiles>

1-(Difluoromethyl)-3,5-dimethoxybenzene (11). The product (41 mg, 73\% yield) as a colorless oil was purified with silica gel chromatography (Petroleum ether/EtOAc $=30: 1) .{ }^{1} \mathrm{H} \mathrm{NMR}(400 \mathrm{MHz}$, $\left.\mathrm{CDCl}_{3}\right) \delta 6.64(\mathrm{~m}, 2 \mathrm{H}), 6.54(\mathrm{~m}, 1 \mathrm{H}), 6.56(\mathrm{t}, J=56.4 \mathrm{~Hz}, 1 \mathrm{H}), 3.82(\mathrm{~s}, 6 \mathrm{H}) .{ }^{19} \mathrm{~F} \mathrm{NMR}(376 \mathrm{MHz}$, $\left.\mathrm{CDCl}_{3}\right) \delta-110.9(\mathrm{~d}, J=56.4 \mathrm{~Hz}, 2 \mathrm{~F}) .{ }^{13} \mathrm{C} \mathrm{NMR}\left(100 \mathrm{MHz}, \mathrm{CDCl}_{3}\right) \delta 161.1,136.4(\mathrm{t}, J=22.0 \mathrm{~Hz})$, $114.5(\mathrm{t}, J=238.1 \mathrm{~Hz}), 103.4(\mathrm{t}, J=6.2 \mathrm{~Hz}), 102.7(\mathrm{t}, J=1.4 \mathrm{~Hz}), 55.5 . \mathrm{MS}(\mathrm{EI}): \mathrm{m} / z(\%) 188\left(\mathrm{M}^{+}\right)$, 188 (100). HRMS calcd. for $\mathrm{C}_{9} \mathrm{H}_{10} \mathrm{O}_{2} \mathrm{~F}_{2}\left(\mathrm{M}^{+}\right)$: 188.0649; Found: 188.0651.<smiles>FC(F)(F)c1ccc2c(c1)OCO2</smiles>

5-(Difluoromethyl)benzo[d][1,3]dioxole (12). The product (34 $\mathrm{mg}, 65 \%$ yield) as a colorless oil was purified with silica gel chromatography (Petroleum ether/EtOAc $=30: 1)$. This compound is known. ${ }^{6}$ ${ }^{1} \mathrm{H}$ NMR $\left(300 \mathrm{MHz}, \mathrm{CDCl}_{3}\right) \delta$ 6.99-6.96 (m, $\left.2 \mathrm{H}\right), 6.84(\mathrm{~d}, J=8.4 \mathrm{~Hz}, 1 \mathrm{H}), 6.55(\mathrm{t}, J=56.4 \mathrm{~Hz}, 1 \mathrm{H})$, $6.02(\mathrm{~s}, 2 \mathrm{H}) .{ }^{19} \mathrm{~F}$ NMR $\left(282 \mathrm{MHz}, \mathrm{CDCl}_{3}\right) \delta-108.3(\mathrm{~d}, J=56.4 \mathrm{~Hz}, 2 \mathrm{~F}) .{ }^{13} \mathrm{C} \mathrm{NMR}\left(100 \mathrm{MHz}, \mathrm{CDCl}_{3}\right)$ $\delta 149.6(\mathrm{t}, J=1.4 \mathrm{~Hz}), 148.1,128.3(\mathrm{t}, J=22.6 \mathrm{~Hz}), 120.1(\mathrm{t}, J=7.2 \mathrm{~Hz}), 114.6(\mathrm{t}, J=236.6 \mathrm{~Hz}), 108.2$, $105.8(\mathrm{t}, J=5.4 \mathrm{~Hz})$, 101.6. MS (EI): $m / z(\%) 172\left(\mathrm{M}^{+}\right), 171(100)$. HRMS calcd. for $\mathrm{C}_{8} \mathrm{H}_{6} \mathrm{O}_{2} \mathrm{~F}_{2}\left(\mathrm{M}^{+}\right)$: 
172.0336; Found: 172.0337.<smiles>FC(F)(F)c1ccc(COc2cccc3ccccc23)cc1</smiles>

1-((4-(Difluoromethyl)benzyl)oxy)naphthalene (13). The product (55 mg, 65\% yield) as a solid (m.p. $76-80{ }^{\circ} \mathrm{C}$ ) was purified with silica gel chromatography (Petroleum ether/EtOAc $\left.=40: 1\right) .{ }^{1} \mathrm{H}$ NMR $(400$ $\left.\mathrm{MHz}, \mathrm{CDCl}_{3}\right) \delta 8.37-8.35(\mathrm{~m}, 1 \mathrm{H}), 7.84-7.82(\mathrm{~m}, 1 \mathrm{H}), 7.63(\mathrm{~d}, J=8.4 \mathrm{~Hz}, 2 \mathrm{H}), 7.57(\mathrm{~d}, J=8.4 \mathrm{~Hz}$, $2 \mathrm{H}), 7.54-7.50(\mathrm{~m}, 2 \mathrm{H}), 7.47$ (d, J=8.4 Hz, $1 \mathrm{H}), 7.37$ (t, $J=8.4 \mathrm{~Hz}, 1 \mathrm{H}), 6.87(\mathrm{~d}, J=7.6 \mathrm{~Hz}, 1 \mathrm{H})$, $6.69(\mathrm{t}, J=56.4 \mathrm{~Hz}, 1 \mathrm{H}), 5.30(\mathrm{~s}, 2 \mathrm{H}) .{ }^{19} \mathrm{~F} \mathrm{NMR}\left(376 \mathrm{MHz}, \mathrm{CDCl}_{3}\right) \delta-110.7(\mathrm{~d}, J=56.4 \mathrm{~Hz}, 2 \mathrm{~F})$. ${ }^{13} \mathrm{C} \mathrm{NMR}\left(100 \mathrm{MHz}, \mathrm{CDCl}_{3}\right) \delta 154.2,140.0(\mathrm{t}, J=2.0 \mathrm{~Hz}), 134.6,133.9(\mathrm{t}, J=22.3 \mathrm{~Hz}), 127.6,127.4$, $126.6,125.9(\mathrm{t}, J=5.9 \mathrm{~Hz}), 125.8(\mathrm{t}, J=7.9 \mathrm{~Hz}), 125.4,122.1,120.8,114.7(\mathrm{t}, J=237.1 \mathrm{~Hz}), 105.2$, 69.4. MS (EI): $m / z(\%) 284\left(\mathrm{M}^{+}\right), 141$ (100). HRMS calcd. for $\mathrm{C}_{1} \mathrm{H}_{14} \mathrm{OF}_{2}\left(\mathrm{M}^{+}\right)$: 284.1013; Found: 284.1010<smiles>Cc1ccc(C)c(C(F)(F)F)c1</smiles>

2-(Difluoromethyl)-1,4-dimethylbenzene (14). The product (19 mg, 41\% yield; ${ }^{19} \mathrm{~F} \mathrm{NMR}$ yield $71 \%$ ) as a colorless oil was purified with silica gel chromatography (Petroleum ether). ${ }^{1} \mathrm{H}$ NMR (300 MHz, $\left.\mathrm{CDCl}_{3}\right) \delta 7.32(\mathrm{~s}, 1 \mathrm{H}), 7.17(\mathrm{~d}, J=7.8 \mathrm{~Hz}, 1 \mathrm{H}), 7.11(\mathrm{~d}, J=7.8 \mathrm{~Hz}, 1 \mathrm{H}), 6.73(\mathrm{t}, J=55.5 \mathrm{~Hz}, 1 \mathrm{H})$, 2.39 (s, $3 \mathrm{H}), 2.35$ (s, $3 \mathrm{H}) .{ }^{19} \mathrm{~F}$ NMR $\left(282 \mathrm{MHz}, \mathrm{CDCl}_{3}\right) \delta-113.3(\mathrm{~d}, J=55.5 \mathrm{~Hz}, 2 \mathrm{~F}) . \mathrm{MS}(\mathrm{EI}): \mathrm{m} / \mathrm{z}$ (\%) $156\left(\mathrm{M}^{+}\right), 141,105$ (100). HRMS calcd. for $\mathrm{C}_{9} \mathrm{H}_{10} \mathrm{~F}_{2}\left(\mathrm{M}^{+}\right)$: 156.0751; Found:156.0747.<smiles>FC(F)(F)c1cccc2ccccc12</smiles>

1-(Difluoromethyl)naphthalene (15). The product (45 $\mathrm{mg}, 84 \%$ yield) as a colorless oil was purified 
with silica gel chromatography (Petroleum ether). This compound is known. ${ }^{7} \mathrm{H}$ NMR (400 MHz, $\left.\mathrm{CDCl}_{3}\right) \delta 8.16(\mathrm{~d}, J=8.4 \mathrm{~Hz}, 1 \mathrm{H}), 7.94(\mathrm{~d}, J=8.4 \mathrm{~Hz}, 1 \mathrm{H}), 7.90(\mathrm{~d}, J=8.4 \mathrm{~Hz}, 1 \mathrm{H}), 7.68(\mathrm{~d}, J=6.8$ $\mathrm{Hz}, 1 \mathrm{H}), 7.60-7.52$ (m, $2 \mathrm{H}), 7.49$ (t, $J=7.6 \mathrm{~Hz}, 1 \mathrm{H}), 7.12(\mathrm{t}, J=55.2 \mathrm{~Hz}, 1 \mathrm{H}) .{ }^{19} \mathrm{~F}$ NMR $(376 \mathrm{MHz}$, $\left.\mathrm{CDCl}_{3}\right) \delta-110.9(\mathrm{~d}, J=55.2 \mathrm{~Hz}, 2 \mathrm{~F}) .{ }^{13} \mathrm{C} \mathrm{NMR}\left(100 \mathrm{MHz}, \mathrm{CDCl}_{3}\right) \delta 133.8,131.5(\mathrm{t}, J=1.6 \mathrm{~Hz})$, $129.7(\mathrm{t}, J=2.8 \mathrm{~Hz}), 129.5(\mathrm{t}, J=20.6 \mathrm{~Hz}), 128.8,127.2,126.4,124.8(\mathrm{t}, J=8.6 \mathrm{~Hz}), 124.7,123.6$, $115.4(\mathrm{t}, J=237.0 \mathrm{~Hz})$. MS (EI): $m / z(\%) 178\left(\mathrm{M}^{+}\right), 178(100)$. HRMS calcd. for $\mathrm{C}_{11} \mathrm{H}_{8} \mathrm{~F}_{2}\left(\mathrm{M}^{+}\right)$: 178.0594; Found: 178.0590.<smiles>FC(F)(F)c1ccc2ccccc2c1</smiles>

2-(Difluoromethyl)naphthalene (16). The product (41 $\mathrm{mg}, 77 \%$ yield) as a white solid was purified with silica gel chromatography (Petroleum ether). This compound is known. ${ }^{6}{ }^{1} \mathrm{H} \mathrm{NMR} \mathrm{(400} \mathrm{MHz,}$ $\left.\mathrm{CDCl}_{3}\right) \delta 7.99(\mathrm{~s}, 1 \mathrm{H})$, , 7.95-7.89 (m, $\left.3 \mathrm{H}\right), 7.62(\mathrm{~d}, J=9.2 \mathrm{~Hz}, 1 \mathrm{H}), 7.59-7.54(\mathrm{~m}, 2 \mathrm{H}), 6.82(\mathrm{t}, J=$ $56.4 \mathrm{~Hz}, 1 \mathrm{H}) .{ }^{19} \mathrm{~F}$ NMR $\left(376 \mathrm{MHz}, \mathrm{CDCl}_{3}\right) \delta-109.9$ (d, $\left.J=56.4 \mathrm{~Hz}, 2 \mathrm{~F}\right) .{ }^{13} \mathrm{C}$ NMR $(125.7 \mathrm{MHz}$, $\left.\mathrm{CDCl}_{3}\right) \delta 134.3(\mathrm{t}, J=1.3 \mathrm{~Hz}), 132.6,131.6(\mathrm{t}, J=22.2 \mathrm{~Hz}), 128.9,128.5,127.9,127.4,126.8,125.9$ $(\mathrm{t}, J=7.7 \mathrm{~Hz}), 122.0(\mathrm{t}, J=4.8 \mathrm{~Hz}), 115.1(\mathrm{t}, J=238.4 \mathrm{~Hz}) . \mathrm{MS}(\mathrm{EI}): m / z(\%) 178\left(\mathrm{M}^{+}\right), 178(100)$. HRMS calcd. for $\mathrm{C}_{11} \mathrm{H}_{8} \mathrm{~F}_{2}\left(\mathrm{M}^{+}\right)$: 178.0594; Found: 178.0596 .<smiles>FC(F)(F)c1cc2ccccc2c2ccccc12</smiles>

9-(Difluoromethyl)phenanthrene (17). The product (49 mg, 72\% yield) as a white solid (m.p. 116$118{ }^{\circ} \mathrm{C}$ ) was purified with silica gel chromatography (Petroleum ether). ${ }^{1} \mathrm{H}$ NMR $\left(400 \mathrm{MHz}, \mathrm{CDCl}_{3}\right)$ $\delta 8.70(\mathrm{dd}, J=8.4 \mathrm{~Hz}, J=1.6 \mathrm{~Hz}, 1 \mathrm{H}), 8.64(\mathrm{~d}, J=8.4 \mathrm{~Hz}, 1 \mathrm{H}), 8.20-8.18(\mathrm{~m}, 1 \mathrm{H}), 7.93(\mathrm{~s}, 1 \mathrm{H})$, $7.89(\mathrm{~d}, J=8.4 \mathrm{~Hz}, 1 \mathrm{H}), 7.72-7.58(\mathrm{~m}, 4 \mathrm{H}), 7.12(\mathrm{t}, J=55.2 \mathrm{~Hz}, 1 \mathrm{H}) .{ }^{19} \mathrm{~F}$ NMR $\left(376 \mathrm{MHz}, \mathrm{CDCl}_{3}\right)$ $\delta$-111.7 (d, $J=55.2 \mathrm{~Hz}, 2 \mathrm{~F}) .{ }^{13} \mathrm{C} \mathrm{NMR}\left(100 \mathrm{MHz}, \mathrm{CDCl}_{3}\right) \delta 131.4,130.9,130.2,129.5,128.3,128.0$ (t, $J=20.5 \mathrm{~Hz}), 127.9(\mathrm{t}, J=1.8 \mathrm{~Hz}), 127.2,127.15,127.13,126.8(\mathrm{t}, J=9.4 \mathrm{~Hz}), 124.5(\mathrm{t}, J=1.6 \mathrm{~Hz})$, 123.3, 122.7, $115.7(\mathrm{t}, J=237.1 \mathrm{~Hz})$. MS (EI): $m / z(\%) 228\left(\mathrm{M}^{+}\right), 228(100)$. HRMS calcd. for $\mathrm{C}_{15} \mathrm{H}_{10} \mathrm{~F}_{2}$ $\left(\mathrm{M}^{+}\right): 228.0751$; Found: 228.0753 
CHO

4-(Difluoromethyl)benzaldehyde (18). The product (27 mg, 57\% yield) as a colorless oil was purified with silica gel chromatography (Petroleum ether/EtOAc $=10: 1)$. This compound is known. ${ }^{1} \mathrm{H}$ NMR $\left(300 \mathrm{MHz}, \mathrm{CDCl}_{3}\right) \delta 10.08(\mathrm{~s}, 1 \mathrm{H}), 7.98(\mathrm{~d}, J=7.8 \mathrm{~Hz}, 2 \mathrm{H}), 7.69(\mathrm{~d}, J=7.8 \mathrm{~Hz}, 2 \mathrm{H}), 6.72(\mathrm{t}, J=$ $56.1 \mathrm{~Hz}, 1 \mathrm{H}) .{ }^{19} \mathrm{~F} \mathrm{NMR}\left(282 \mathrm{MHz}, \mathrm{CDCl}_{3}\right) \delta-112.9$ (d, $\left.J=56.1 \mathrm{~Hz}, 2 \mathrm{~F}\right) .{ }^{13} \mathrm{C} \mathrm{NMR}\left(100 \mathrm{MHz}, \mathrm{CDCl}_{3}\right)$ $\delta 191.5,139.8(\mathrm{t}, J=22.3 \mathrm{~Hz}), 137.9,129.9,126.3(\mathrm{t}, J=6.1 \mathrm{~Hz}), 113.8(\mathrm{t}, J=238.6 \mathrm{~Hz}) . \mathrm{MS}(\mathrm{EI})$ : $m / z(\%) 156\left(\mathrm{M}^{+}\right), 156(100)$. HRMS calcd. for $\mathrm{C}_{8} \mathrm{H}_{6} \mathrm{OF}_{2}\left(\mathrm{M}^{+}\right)$: 156.0387; Found: 156.0384.<smiles>O=Cc1cccc(C(F)(F)F)c1</smiles>

3-(Difluoromethyl)benzaldehyde (19). The product (26 mg, 55\% yield) as a colorless oil was purified with silica gel chromatography (Petroleum ether). ${ }^{1} \mathrm{H}$ NMR (400 MHz, $\left.\mathrm{CDCl}_{3}\right) \delta 10.06$ (s, $\left.1 \mathrm{H}\right), 8.02$ (s, $1 \mathrm{H}), 7.99(\mathrm{~d}, J=7.6 \mathrm{~Hz}, 1 \mathrm{H}), 7.78(\mathrm{~d}, J=7.6 \mathrm{~Hz}, 1 \mathrm{H}), 6.65(\mathrm{t}, J=7.6 \mathrm{~Hz}, 1 \mathrm{H}), 6.72(\mathrm{t}, J=56.4$ $\mathrm{Hz}, 1 \mathrm{H}) .{ }^{19} \mathrm{~F}$ NMR $\left(376 \mathrm{MHz}, \mathrm{CDCl}_{3}\right) \delta-111.7(\mathrm{~d}, J=56.4 \mathrm{~Hz}, 2 \mathrm{~F}) .{ }^{13} \mathrm{C} \mathrm{NMR}\left(100 \mathrm{MHz}, \mathrm{CDCl}_{3}\right) \delta$ 191.3, 136.6, $135.5(\mathrm{t}, J=23.1 \mathrm{~Hz}), 131.8(\mathrm{t}, J=1.4 \mathrm{~Hz}), 131.3(\mathrm{t}, J=5.7 \mathrm{~Hz}), 129.6,126.8(\mathrm{t}, J=6.3$ $\mathrm{Hz}), 113.8(\mathrm{t}, J=238.4 \mathrm{~Hz})$. MS (EI): $m / z$ (\%) $156\left(\mathrm{M}^{+}\right), 155(100)$. HRMS calcd. for $\mathrm{C}_{8} \mathrm{H}_{6} \mathrm{OF}_{2}\left(\mathrm{M}^{+}\right)$: 156.0387; Found: 156.0390.<smiles>O=Cc1ccccc1C(F)(F)F</smiles>

2-(Difluoromethyl)benzaldehyde (20). The product (25 mg, 52\% yield) as a colorless oil was purified with silica gel chromatography (Petroleum ether/EtOAc $=10: 1)$. This compound is known. ${ }^{1} \mathrm{H}$ NMR $\left(300 \mathrm{MHz}, \mathrm{CDCl}_{3}\right) \delta 10.18$ (s, $\left.1 \mathrm{H}\right)$, 7.95-7.93 (m, $\left.1 \mathrm{H}\right)$, 7.83-7.81 (m, $\left.1 \mathrm{H}\right)$, 7.75-7.70 (m, $\left.2 \mathrm{H}\right), 7.43$ $(\mathrm{t}, J=54.9 \mathrm{~Hz}, 1 \mathrm{H}) .{ }^{19} \mathrm{~F}$ NMR $\left(282 \mathrm{MHz}, \mathrm{CDCl}_{3}\right) \delta-112.1(\mathrm{~d}, J=54.9 \mathrm{~Hz}, 2 \mathrm{~F}) .{ }^{13} \mathrm{C} \mathrm{NMR}(125.7$ $\left.\mathrm{MHz}, \mathrm{CDCl}_{3}\right) \delta 191.7,134.6,134.1,133.4,131.1(\mathrm{t}, J=1.9 \mathrm{~Hz}), 128.9,126.5(\mathrm{t}, J=8.3 \mathrm{~Hz}), 111.9(\mathrm{t}$, 
$J=238.2 \mathrm{~Hz}$ ). IR (thin film) $v_{\max } 2959,2926,1732,1715 \mathrm{~cm}^{-1} . \mathrm{MS}(\mathrm{EI}): \mathrm{m} / z(\%) 156\left(\mathrm{M}^{+}\right), 155(100)$. HRMS calcd. for $\mathrm{C}_{8} \mathrm{H}_{6} \mathrm{OF}_{2}\left(\mathrm{M}^{+}\right)$: 156.0387 ; Found: 156.0385 .<smiles>CCOC(=O)c1ccc(C(F)(F)F)cc1</smiles>

Ethyl 4-(difluoromethyl)benzoate (21). The product (48 $\mathrm{mg}, 80 \%$ yield) as a colorless oil was purified with silica gel chromatography (Petroleum ether/EtOAc $=10: 1$ ). This compound is known. ${ }^{6}$ ${ }^{1} \mathrm{H}$ NMR $\left(300 \mathrm{MHz}, \mathrm{CDCl}_{3}\right) \delta 8.12(\mathrm{~d}, J=8.4 \mathrm{~Hz}, 2 \mathrm{H}), 7.57(\mathrm{~d}, J=8.4 \mathrm{~Hz}, 2 \mathrm{H}), 6.69(\mathrm{t}, J=56.1 \mathrm{~Hz}$, $1 \mathrm{H}), 4.39(\mathrm{q}, J=7.2 \mathrm{~Hz}, 2 \mathrm{H}), 1.40(\mathrm{t}, J=7.2 \mathrm{~Hz}, 3 \mathrm{H}) .{ }^{19} \mathrm{~F}$ NMR $\left(376 \mathrm{MHz}, \mathrm{CDCl}_{3}\right) \delta-112.2(\mathrm{~d}, J=$ $56.1 \mathrm{~Hz}, 2 \mathrm{~F}) .{ }^{13} \mathrm{C}$ NMR $\left(100 \mathrm{MHz}, \mathrm{CDCl}_{3}\right) \delta 165.8,138.3(\mathrm{t}, J=22.3 \mathrm{~Hz}), 132.6,129.9,125.6(\mathrm{t}, J=$ $5.9 \mathrm{~Hz}), 114.0(\mathrm{t}, J=238.4 \mathrm{~Hz}), 61.4,14.3$. IR (thin film) $v_{\max } 2983,2930,1767,1723 \mathrm{~cm}^{-1}$. MS (EI): $m / z(\%) 200\left(\mathrm{M}^{+}\right)$, 199, 172, 155 (100). HRMS calcd. for $\mathrm{C}_{10} \mathrm{H}_{10} \mathrm{O}_{2} \mathrm{~F}_{2}\left(\mathrm{M}^{+}\right)$: 200.0649; Found: 200.0648 .<smiles>CCOC(=O)c1cccc(C(F)(F)F)c1</smiles>

Ethyl 3-(difluoromethyl)benzoate (22). The product (45 $\mathrm{mg}, 75 \%$ yield) as a colorless oil was purified with silica gel chromatography (Petroleum ether/EtOAc $=10: 1) .{ }^{1} \mathrm{H}$ NMR $\left(400 \mathrm{MHz}, \mathrm{CDCl}_{3}\right)$ $\delta 8.18-8.15(\mathrm{~m}, 2 \mathrm{H}), 7.71(\mathrm{~d}, J=7.6 \mathrm{~Hz}, 1 \mathrm{H}), 7.55(\mathrm{t}, J=7.6 \mathrm{~Hz}, 1 \mathrm{H}), 6.69$ (t, $J=56.0 \mathrm{~Hz}, 1 \mathrm{H})$, $4.41(\mathrm{q}, J=6.8 \mathrm{~Hz}, 2 \mathrm{H}), 1.41(\mathrm{t}, J=6.8 \mathrm{~Hz}, 3 \mathrm{H}) .{ }^{19} \mathrm{~F}$ NMR $\left(376 \mathrm{MHz}, \mathrm{CDCl}_{3}\right) \delta-111.1(\mathrm{~d}, J=56.0$ $\mathrm{Hz}, 2 \mathrm{~F}) .{ }^{13} \mathrm{C} \mathrm{NMR}\left(100 \mathrm{MHz}, \mathrm{CDCl}_{3}\right) \delta 165.7,134.7$ (t, $\left.J=22.5 \mathrm{~Hz}\right), 131.7$ (t, $\left.J=1.3 \mathrm{~Hz}\right), 131.1$, $129.7(\mathrm{t}, J=5.6 \mathrm{~Hz}), 128.9,126.9(\mathrm{t}, J=6.1 \mathrm{~Hz}), 114.2(\mathrm{t}, J=238.1 \mathrm{~Hz}), 61.4,14.3$. IR (thin film) $v_{\max }$ 2983, 1766, $1615 \mathrm{~cm}^{-1}$. MS (EI): $\mathrm{m} / z(\%) 200\left(\mathrm{M}^{+}\right), 193,172,155$ (100). HRMS calcd. for $\mathrm{C}_{10} \mathrm{H}_{10} \mathrm{O}_{2} \mathrm{~F}_{2}$ $\left(\mathrm{M}^{+}\right)$: 200.0649; Found: 200.0652. 
COMe

1-(4-(Difluoromethyl)phenyl)ethanone (23). The product (28 $\mathrm{mg}, 55 \%$ yield) as a colorless oil was purified with silica gel chromatography (Petroleum ether/EtOAc $=10: 1)$. This compound is known. ${ }^{7}$ ${ }^{1} \mathrm{H}$ NMR $\left(300 \mathrm{MHz}, \mathrm{CDCl}_{3}\right) \delta 8.02(\mathrm{~d}, J=8.1 \mathrm{~Hz}, 2 \mathrm{H}), 7.59(\mathrm{~d}, J=8.1 \mathrm{~Hz}, 2 \mathrm{H}), 6.68(\mathrm{t}, J=56.1 \mathrm{~Hz}$, $1 \mathrm{H}), 2.62(\mathrm{~s}, 3 \mathrm{H}) .{ }^{19} \mathrm{~F} \mathrm{NMR}\left(282 \mathrm{MHz}, \mathrm{CDCl}_{3}\right) \delta-112.7(\mathrm{~d}, J=56.1 \mathrm{~Hz}, 2 \mathrm{~F}) .{ }^{13} \mathrm{C} \mathrm{NMR}(100 \mathrm{MHz}$, $\left.\mathrm{CDCl}_{3}\right) \delta 197.4,138.8,138.5(\mathrm{t}, J=22.1 \mathrm{~Hz}), 128.6,125.9(\mathrm{t}, J=6.0 \mathrm{~Hz}), 113.9(\mathrm{t}, J=238.2 \mathrm{~Hz}), 26.8$. IR (thin film) $v_{\max } 3064,2967,1767,1689 \mathrm{~cm}^{-1}$. MS (EI): $\mathrm{m} / z(\%) 170\left(\mathrm{M}^{+}\right), 155$ (100). HRMS calcd. for $\mathrm{C}_{9} \mathrm{H}_{8} \mathrm{OF}_{2}\left(\mathrm{M}^{+}\right)$: 170.0543; Found: 170.0542 .<smiles>CC(=O)c1cccc(C(F)(F)F)c1</smiles>

1-(3-(Difluoromethyl)phenyl)ethanone (24). The product (37 $\mathrm{mg}, 73 \%$ yield) was purified with silica gel chromatography (Petroleum ether/EtOAc = 8:1). ${ }^{1} \mathrm{H}$ NMR $\left(400 \mathrm{MHz}, \mathrm{CDCl}_{3}\right) \delta 8.08(\mathrm{~s}, 1 \mathrm{H}), 8.04$ $(\mathrm{d}, J=7.6 \mathrm{~Hz}, 1 \mathrm{H}), 7.69(\mathrm{~d}, J=7.6 \mathrm{~Hz}, 1 \mathrm{H}), 7.55(\mathrm{t}, J=7.6 \mathrm{~Hz}, 1 \mathrm{H}), 6.68(\mathrm{t}, J=56.0 \mathrm{~Hz}, 1 \mathrm{H}), 2.61$ $(\mathrm{s}, 3 \mathrm{H}) .{ }^{19} \mathrm{~F}$ NMR $\left(376 \mathrm{MHz}, \mathrm{CDCl}_{3}\right) \delta-111.2(\mathrm{~d}, J=56.0 \mathrm{~Hz}, 2 \mathrm{~F}) .{ }^{13} \mathrm{C} \mathrm{NMR}\left(100 \mathrm{MHz}, \mathrm{CDCl}_{3}\right) \delta$ 197.1, 137.5, $134.9(\mathrm{t}, J=22.8 \mathrm{~Hz}), 130.5,129.9(\mathrm{t}, J=5.8 \mathrm{~Hz}), 129.2,125.5(\mathrm{t}, J=6.3 \mathrm{~Hz}), 114.1(\mathrm{t}$, $J=238.0 \mathrm{~Hz}), 26.7$. MS (EI): $m / z(\%) 170\left(\mathrm{M}^{+}\right), 155(100)$. HRMS calcd. for $\mathrm{C}_{9} \mathrm{H}_{8} \mathrm{OF}_{2}\left(\mathrm{M}^{+}\right): 170.0543$; Found:170.0542.<smiles>N#Cc1cccc(C(F)(F)F)c1</smiles>

3-(Difluoromethyl)benzonitrile (25). The reaction was conducted in $0.6 \mathrm{mmol}$ scale. The product (41 $\mathrm{mg}, 45 \%$ yield) as a colorless oil was purified with silica gel chromatography (Petroleum ether/EtOAc $=10: 1)$. This compound is known. ${ }^{8}{ }^{1} \mathrm{H} \mathrm{NMR}\left(400 \mathrm{MHz}, \mathrm{CDCl}_{3}\right) \delta 7.81(\mathrm{~s}, 1 \mathrm{H}), 7.77(\mathrm{t}, J=8.0 \mathrm{~Hz}, 2$ $\mathrm{H}), 7.60(\mathrm{t}, J=8.0 \mathrm{~Hz}, 1 \mathrm{H}), 6.68(\mathrm{t}, J=56.1 \mathrm{~Hz}, 1 \mathrm{H}) .{ }^{19} \mathrm{~F} \mathrm{NMR}\left(376 \mathrm{MHz}, \mathrm{CDCl}_{3}\right) \delta-112.8(\mathrm{~d}, J=$ $56.1 \mathrm{~Hz}, 2 \mathrm{~F}) .{ }^{13} \mathrm{C} \mathrm{NMR}\left(125.7 \mathrm{MHz}, \mathrm{CDCl}_{3}\right) \delta 134.2(\mathrm{t}, J=1.5 \mathrm{~Hz}), 129.9(\mathrm{t}, J=5.9 \mathrm{~Hz}), 129.8,129.3$ 
(t, $J=6.2 \mathrm{~Hz}), 117.8(\mathrm{t}, J=12.7 \mathrm{~Hz}), 113.2,113.1(\mathrm{t}, J=240.3 \mathrm{~Hz}), 109.9$. IR (thin film) $v_{\max } 2924$, 2234, $1766 \mathrm{~cm}^{-1}$. MS (EI): $\mathrm{m} / z(\%) 153\left(\mathrm{M}^{+}\right), 152(100)$. HRMS calcd. for $\mathrm{C}_{8} \mathrm{H}_{5} \mathrm{NF}_{2}\left(\mathrm{M}^{+}\right):$153.0390; Found: 153.0388.<smiles>FC(F)(F)c1ccc(C(F)(F)F)cc1</smiles>

1-(Difluoromethyl)-4-(trifluoromethyl)benzene (26). Due to the low biol point of the product, the yield (84\%) was determined by ${ }^{19} \mathrm{~F}$ NMR using fluorobenzene as an internal standard. This compound is known. ${ }^{6}$ The product was characterized by ${ }^{19} \mathrm{~F}$ NMR and GC-MS analysis.<smiles>FC(F)(F)c1cccc(C(F)(F)F)c1</smiles>

1-(Difluoromethyl)-3-(trifluoromethyl)benzene (27). Due to the low biol point of the product, the yield (78\% yield) was determined by ${ }^{19} \mathrm{~F}$ NMR using fluorobenzene as an internal standard. The product was characterized by ${ }^{19} \mathrm{~F}$ NMR and GC-MS analysis.<smiles>FC(F)(F)c1cc(Cl)cc(Cl)c1</smiles>

1,3-dichloro-5-(difluoromethyl)benzene (28). The product (26 mg, 43\% yield; ${ }^{19} \mathrm{~F}$ NMR yield 54\%) was purified with silica gel chromatography (Petroleum ether). This compound is known. ${ }^{9} \mathrm{H}$ NMR $\left(300 \mathrm{MHz}, \mathrm{CDCl}_{3}\right) \delta 7.46(\mathrm{~m}, 1 \mathrm{H}), 7.39(\mathrm{~s}, 2 \mathrm{H}), 6.58(\mathrm{t}, J=56.1 \mathrm{~Hz}, 1 \mathrm{H}) .{ }^{19} \mathrm{~F} \mathrm{NMR}(282 \mathrm{MHz}$, $\left.\mathrm{CDCl}_{3}\right) \delta-112.7(\mathrm{~d}, J=56.1 \mathrm{~Hz}, 2 \mathrm{~F}) . \mathrm{MS}(\mathrm{EI}): m / z(\%) 195\left(\mathrm{M}^{+}\right), 186,177,161$ (100). HRMS calcd. for $\mathrm{C}_{7} \mathrm{H}_{4} \mathrm{Cl}_{2} \mathrm{~F}_{2}\left(\mathrm{M}^{+}\right)$: 195.9658; Found:195.9653.<smiles>FC(F)(F)c1ccccc1Br</smiles> 
1-Bromo-2-(difluoromethyl)benzene (29). The product (20 mg, 33\% yield; ${ }^{19} \mathrm{~F}$ NMR yield 38\%) as a colorless oil was purified with silica gel chromatography (Petroleum ether). This compound is known. ${ }^{10}{ }^{1} \mathrm{H}$ NMR $\left(400 \mathrm{MHz}, \mathrm{CDCl}_{3}\right) \delta 7.66(\mathrm{~d}, J=7.6 \mathrm{~Hz}, 1 \mathrm{H}), 7.62-7.60(\mathrm{~m}, 1 \mathrm{H}), 7.43(\mathrm{t}, J=7.6$ $\mathrm{Hz}, 1 \mathrm{H}), 7.34$ (t, $J=7.6 \mathrm{~Hz}, 1 \mathrm{H}), 6.92(\mathrm{t}, J=54.8 \mathrm{~Hz}, 1 \mathrm{H}) .{ }^{19} \mathrm{~F}$ NMR $\left(375 \mathrm{MHz}, \mathrm{CDCl}_{3}\right) \delta-114.6$ (d, $J=54.8 \mathrm{~Hz}, 2 \mathrm{~F}) . \mathrm{MS}(\mathrm{EI}): \mathrm{m} / z(\%) 206\left(\mathrm{M}^{+}\right), 206(100)$. HRMS calcd. for $\mathrm{C}_{7} \mathrm{H}_{5} \mathrm{BrF}_{2}\left(\mathrm{M}^{+}\right)$: 205.9543; Found: 205.9540.<smiles>CS(=O)(=O)c1ccc(C(F)(F)F)cc1</smiles>

(4-(Difluoromethyl)phenyl)trimethylsilane (30). The product (43 $\mathrm{mg}$, $72 \%$ yield) was purified with silica gel chromatography (Petroleum ether/EtOAc $=30: 1)$. This compound is known. ${ }^{6} \mathrm{H}$ NMR $(300$ $\mathrm{MHz}^{\mathrm{CDCl}} \mathrm{CD}_{3} \delta 7.62(\mathrm{~d}, J=7.5 \mathrm{~Hz}, 2 \mathrm{H}), 7.49(\mathrm{~d}, J=7.5 \mathrm{~Hz}, 2 \mathrm{H}), 6.64(\mathrm{t}, J=56.7 \mathrm{~Hz}, 1 \mathrm{H}), 0.3(\mathrm{~s}$, $9 \mathrm{H}) .{ }^{19} \mathrm{~F}$ NMR $\left(282 \mathrm{MHz}, \mathrm{CDCl}_{3}\right) \delta-111.2(\mathrm{~d}, J=56.7 \mathrm{~Hz}, 2 \mathrm{~F}) .{ }^{13} \mathrm{C} \mathrm{NMR}\left(125.7 \mathrm{MHz}, \mathrm{CDCl}_{3}\right) \delta$ 143.9, $134.6(\mathrm{t}, J=22.2 \mathrm{~Hz}), 133.6,124.7(\mathrm{t}, J=6.0 \mathrm{~Hz}), 114.8(\mathrm{t}, J=238.5 \mathrm{~Hz}),-1.3$. MS (EI): $\mathrm{m} / z$ (\%) $200\left(\mathrm{M}^{+}\right), 185$ (100). HRMS calcd. for $\mathrm{C}_{10} \mathrm{H}_{14} \mathrm{SiF}_{2}\left(\mathrm{M}^{+}\right)$: 200.0833; Found: 200.0838 .<smiles>CCSc1ccc(C(F)(F)F)cc1</smiles>

(4-(Difluoromethyl)phenyl)(ethyl)sulfane (31). The product (49 $\mathrm{mg}, 87 \%$ yield) was purified with silica gel chromatography (Petroleum ether/EtOAc $=30: 1) .{ }^{1} \mathrm{H}$ NMR $\left(300 \mathrm{MHz}, \mathrm{CDCl}_{3}\right) \delta 7.41(\mathrm{~d}, J$ $=8.4 \mathrm{~Hz}, 2 \mathrm{H}), 7.35(\mathrm{~d}, J=8.4 \mathrm{~Hz}, 2 \mathrm{H}), 6.61(\mathrm{t}, J=56.7 \mathrm{~Hz}, 1 \mathrm{H}), 2.98(\mathrm{q}, J=7.5 \mathrm{~Hz}, 2 \mathrm{H}), 1.35(\mathrm{t}$, $J=7.5 \mathrm{~Hz}, 3 \mathrm{H}) .{ }^{19} \mathrm{~F}$ NMR $\left(282 \mathrm{MHz}, \mathrm{CDCl}_{3}\right) \delta-110.5(\mathrm{~d}, J=56.7 \mathrm{~Hz}, 2 \mathrm{~F}) .{ }^{13} \mathrm{C} \mathrm{NMR}(125.7 \mathrm{MHz}$, $\left.\mathrm{CDCl}_{3}\right) \delta 140.7(\mathrm{t}, J=2.0 \mathrm{~Hz}), 131.3(\mathrm{t}, J=22.4 \mathrm{~Hz}), 127.8,125.9(\mathrm{t}, J=5.9 \mathrm{~Hz}), 114.6(\mathrm{t}, J=238.3$ $\mathrm{Hz}), 26.8,14.1$. MS (EI): $m / z(\%) 188\left(\mathrm{M}^{+}\right), 160$ (100). HRMS calcd. for $\mathrm{C}_{9} \mathrm{H}_{10} \mathrm{SF}_{2}\left(\mathrm{M}^{+}\right): 188.0471$; Found: 188.0469. 
$\underbrace{C F_{2} \mathrm{H}}_{\mathrm{OH}}$

(4-(Difluoromethyl)phenyl)methanol (32). The product (36 mg, 76\% yield) as a colorless oil was purified with silica gel chromatography (Petroleum ether/EtOAc $=3: 1$ ). This compound is known. ${ }^{11}$ ${ }^{1} \mathrm{H}$ NMR $\left(400 \mathrm{MHz}, \mathrm{CDCl}_{3}\right) \delta 7.51(\mathrm{~d}, J=8.4 \mathrm{~Hz}, 2 \mathrm{H}), 7.45(\mathrm{~d}, J=8.4 \mathrm{~Hz}, 2 \mathrm{H}), 6.65(\mathrm{t}, J=56.4 \mathrm{~Hz}$, $1 \mathrm{H}), 4.76(\mathrm{~s}, 2 \mathrm{H}), 1.76 \mathrm{~s}, 1 \mathrm{H}) .{ }^{19} \mathrm{~F}$ NMR $\left(376 \mathrm{MHz} \mathrm{CDCl}_{3}\right) \delta-110.4(\mathrm{~d}, J=56.4 \mathrm{~Hz}, 2 \mathrm{~F}) . \mathrm{MS}(\mathrm{EI})$ : $m / z(\%) 158\left(\mathrm{M}^{+}\right), 127,107$ (100). IR (thin film) $v_{\max }$ 3332, 1759, 1620, $1508 \mathrm{~cm}^{-1}$. HRMS calcd. for $\mathrm{C}_{8} \mathrm{H}_{8} \mathrm{OF}_{2}\left(\mathrm{M}^{+}\right): 158.0543$; Found: 158.0540 .

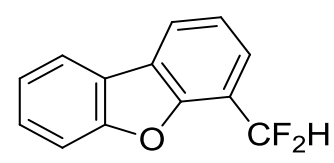

4-(Difluoromethyl)dibenzo[b,d]furan (33). The product (50 $\mathrm{mg}, 77 \%$ yield) as a colorless oil was purified with silica gel chromatography (Petroleum ether). ${ }^{1} \mathrm{H}$ NMR $\left(400 \mathrm{MHz}, \mathrm{CDCl}_{3}\right) \delta 8.02(\mathrm{dd}, J$ $=7.6 \mathrm{~Hz}, J=0.8 \mathrm{~Hz}, 1 \mathrm{H}), 7.94(\mathrm{~d}, J=7.6 \mathrm{~Hz}, 1 \mathrm{H}), 7.67(\mathrm{~d}, J=7.6 \mathrm{~Hz}, 1 \mathrm{H}), 7.62(\mathrm{~d}, J=8.4 \mathrm{~Hz}, 1$ H), $7.50(\mathrm{td}, J=8.4 \mathrm{~Hz}, J=1.2 \mathrm{~Hz}, 1 \mathrm{H}), 7.41(\mathrm{t}, J=8.0 \mathrm{~Hz}, 1 \mathrm{H}), 7.38(\mathrm{t}, J=8.0 \mathrm{~Hz}, 1 \mathrm{H}), 7.23(\mathrm{t}, J$ $=55.2 \mathrm{~Hz}, 1 \mathrm{H}) .{ }^{19} \mathrm{~F}$ NMR $\left(376 \mathrm{MHz}, \mathrm{CDCl}_{3}\right) \delta-113.0(\mathrm{~d}, J=55.2 \mathrm{~Hz}, 2 \mathrm{~F}) .{ }^{13} \mathrm{C} \mathrm{NMR}(125.7 \mathrm{MHz}$, $\left.\mathrm{CDCl}_{3}\right) \delta 156.3,127.8,125.1,123.7(\mathrm{t}, J=5.8 \mathrm{~Hz}), 123.4,123.2,123.1(\mathrm{t}, J=1.6 \mathrm{~Hz}), 122.7,120.8$, $118.5(\mathrm{t}, J=24.0 \mathrm{~Hz}), 111.9,111.8(\mathrm{t}, J=237.4 \mathrm{~Hz}) . \mathrm{MS}(\mathrm{EI}): m / z(\%) 218\left(\mathrm{M}^{+}\right), 218(100) . \mathrm{HRMS}$ calcd. for $\mathrm{C}_{13} \mathrm{H}_{8} \mathrm{OF}_{2}\left(\mathrm{M}^{+}\right)$: 218.0543; Found: 218.0538 .

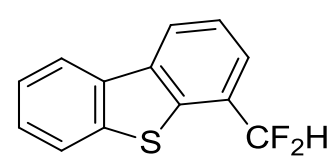

4-(Difluoromethyl)dibenzo[b,d]thiophene (34). The product (55 $\mathrm{mg}, 79 \%$ yield) as a colorless oil was purified with silica gel chromatography (Petroleum ether). ${ }^{1} \mathrm{H}$ NMR $\left(300 \mathrm{MHz}, \mathrm{CDCl}_{3}\right) \delta 8.23-$ 8.20 (m, 1 H), 8.17-8.14 (m, 1 H), 7.89-7.86 (m, 1 H), 7.63-7.60 (m, 1 H), 7.53-7.47 (m, 3 H), 6.93 (t, $J=55.4 \mathrm{~Hz}, 1 \mathrm{H}) .{ }^{19} \mathrm{~F}$ NMR $\left(282 \mathrm{MHz}, \mathrm{CDCl}_{3}\right) \delta-113.7(\mathrm{~d}, J=55.4 \mathrm{~Hz}, 2 \mathrm{~F}) .{ }^{13} \mathrm{C} \mathrm{NMR}(125.7 \mathrm{MHz}$, $\left.\mathrm{CDCl}_{3}\right) \delta 139.5(\mathrm{t}, J=1.0 \mathrm{~Hz}), 136.9,136.6(\mathrm{t}, J=3.3 \mathrm{~Hz}), 134.6,128.5(\mathrm{t}, J=22.8 \mathrm{~Hz}), 127.3,124.7$, 124.4, 124.3 (t, $J=7.0 \mathrm{~Hz}), 123.7(\mathrm{t}, J=1.8 \mathrm{~Hz}), 122.7,121.7,114.5(\mathrm{t}, J=239.4 \mathrm{~Hz}) . \mathrm{MS}(\mathrm{EI}): \mathrm{m} / \mathrm{z}$ 
(\%) $234\left(\mathrm{M}^{+}\right), 234$ (100). HRMS calcd. for $\mathrm{C}_{13} \mathrm{H}_{8} \mathrm{SF}_{2}\left(\mathrm{M}^{+}\right)$: 234.0315; Found: 234.0320. IR (thin film) $v_{\max } 3067,2963,1457 \mathrm{~cm}^{-1}$.<smiles>FC(F)(F)c1ccc2[nH]c3ccccc3c2c1</smiles>

3-(Difluoromethyl)-9-phenyl-9H-carbazole (35). The product (49 $\mathrm{mg}, 56 \%$ yield) as a colorless oil was purified with silica gel chromatography (Petroleum ether). ${ }^{1} \mathrm{H} \mathrm{NMR}\left(400 \mathrm{MHz}, \mathrm{CDCl}_{3}\right) \delta 8.32$ (s, $1 \mathrm{H}), 8.18(\mathrm{~d}, J=8.0 \mathrm{~Hz}, 1 \mathrm{H}), 7.64(\mathrm{t}, J=6.8 \mathrm{~Hz}, 2 \mathrm{H}), 7.57-7.55(\mathrm{~m}, 3 \mathrm{H}), 7.52-7.42(\mathrm{~m}, 4 \mathrm{H}), 7.35$ $(\mathrm{t}, J=7.2 \mathrm{~Hz}, 1 \mathrm{H}), 6.88(\mathrm{t}, J=56.8 \mathrm{~Hz}, 1 \mathrm{H}) .{ }^{19} \mathrm{~F} \mathrm{NMR}\left(376 \mathrm{MHz}, \mathrm{CDCl}_{3}\right) \delta-106.3(\mathrm{~d}, J=56.8 \mathrm{~Hz}$, $2 \mathrm{~F}) .{ }^{13} \mathrm{C} \mathrm{NMR}\left(125.7 \mathrm{MHz}, \mathrm{CDCl}_{3}\right) \delta 142.0,141.5,137.2,130.0,127.9,127.2,126.6,126.1(\mathrm{t}, J=$ $22.4 \mathrm{~Hz}), 123.2$ (t, $J=5.7 \mathrm{~Hz}), 123.1,122.9,120.49,120.48,118.1$ (t, $J=6.4 \mathrm{~Hz}), 115.8$ (t, $J=237.3$ $\mathrm{Hz}), 110.1$, 109.9. MS (EI): $\mathrm{m} / z$ (\%) $292\left(\mathrm{M}^{+}-\mathrm{H}\right), 271$ (100). HRMS calcd. for $\mathrm{C}_{19} \mathrm{H}_{13} \mathrm{NF}_{2}\left(\mathrm{M}^{+}\right)$: 293.1016; Found: 293.1017. IR (thin film) $v_{\max } 3062,1761,1686 \mathrm{~cm}^{-1}$.<smiles>FC(F)(F)c1ccc(N2CCOCC2)cc1</smiles>

4-(4-(Difluoromethyl)phenyl)morpholine (36). The product (40 $\mathrm{mg}, 62 \%$ yield) as a colorless oil was purified with silica gel chromatography (Petroleum ether/EtOAc $=3: 1) .{ }^{1} \mathrm{H}$ NMR $(400 \mathrm{MHz}$, $\left.\mathrm{CDCl}_{3}\right) \delta 7.41(\mathrm{~d}, J=8.8 \mathrm{~Hz}, 2 \mathrm{H}), 6.93(\mathrm{~d}, J=8.8 \mathrm{~Hz}, 2 \mathrm{H}), 6.58(\mathrm{t}, J=57.2 \mathrm{~Hz}, 1 \mathrm{H}), 3.87(\mathrm{t}, J=4.8$ $\mathrm{Hz}, 4 \mathrm{H}), 3.21(\mathrm{t}, J=4.8 \mathrm{~Hz}, 4 \mathrm{H}) .{ }^{19} \mathrm{~F} \mathrm{NMR}\left(375 \mathrm{MHz}, \mathrm{CDCl}_{3}\right) \delta-108.1(\mathrm{~d}, J=57.2 \mathrm{~Hz}, 2 \mathrm{~F}) .{ }^{13} \mathrm{C}$ NMR (125.7 MHz, $\left.\mathrm{CDCl}_{3}\right) \delta 152.9(\mathrm{t}, J=1.5 \mathrm{~Hz}), 126.7(\mathrm{t}, J=5.9 \mathrm{~Hz}), 125.3(\mathrm{t}, J=22.9 \mathrm{~Hz}), 115.1$ (t, $J=236.8 \mathrm{~Hz}), 114.8,66.7,48.5 . \mathrm{MS}(\mathrm{EI}): \mathrm{m} / \mathrm{z}(\%) 213\left(\mathrm{M}^{+}\right), 206,191$ (100). HRMS calcd. for $\mathrm{C}_{11} \mathrm{H}_{13} \mathrm{NOF}_{2}\left(\mathrm{M}^{+}\right)$: 213.0965; Found: 213.0970. IR (thin film) $v_{\max } 2963,2853,1686,1600 \mathrm{~cm}^{-1}$. 
<smiles>O=C1CC(c2ccc(C(F)(F)F)cc2)Oc2ccccc21</smiles>

(2-(4-(Difluoromethyl)phenyl)chroman-4-one (37). The product (63 $\mathrm{mg}, 76 \%$ yield) as a white solid (m.p. 61-63 ${ }^{\circ} \mathrm{C}$ ) was purified with silica gel chromatography (Petroleum ether/EtOAc = 3:1). ${ }^{1} \mathrm{H}$ NMR $\left(400 \mathrm{MHz}, \mathrm{CDCl}_{3}\right) \delta 7.93(\mathrm{dd}, J=8.4 \mathrm{~Hz}, J=0.8 \mathrm{~Hz}, 1 \mathrm{H}), 7.59(\mathrm{~s}, 4 \mathrm{H}), 7.52(\mathrm{td}, J=8.4 \mathrm{~Hz}, J=1.6$ $\mathrm{Hz}, 1 \mathrm{H}), 7.08-7.05(\mathrm{~m}, 2 \mathrm{H}), 6.67(\mathrm{t}, J=56.4 \mathrm{~Hz}, 1 \mathrm{H}), 5.53(\mathrm{dd}, J=12.8 \mathrm{~Hz}, J=2.8 \mathrm{~Hz}, 1 \mathrm{H}), 3.06$ $(\mathrm{dd}, J=16.8 \mathrm{~Hz}, J=13.2 \mathrm{~Hz}, 1 \mathrm{H}), 2.90(\mathrm{dd}, J=16.8 \mathrm{~Hz}, J=2.8 \mathrm{~Hz}, 1 \mathrm{H}) .{ }^{19} \mathrm{~F} \mathrm{NMR}(376 \mathrm{MHz}$, $\left.\mathrm{CDCl}_{3}\right) \delta-110.9(\mathrm{~d}, J=56.4 \mathrm{~Hz}, 2 \mathrm{~F}) .{ }^{13} \mathrm{C} \mathrm{NMR}\left(125.7 \mathrm{MHz}, \mathrm{CDCl}_{3}\right) \delta 191.4,161.3,141.5(\mathrm{t}, J=1.9$ $\mathrm{Hz}), 136.3,134.7(\mathrm{t}, J=22.6 \mathrm{~Hz}), 127.1,126.4,126.1(\mathrm{t}, J=6.2 \mathrm{~Hz}), 121.9,120.9,118.1,114.4(\mathrm{t}, J$ $=238.8 \mathrm{~Hz}$ ), 79.0, 44.6. IR (thin film) $v_{\max } 3066,1763,1690,1606 \mathrm{~cm}^{-1} . \mathrm{MS}(\mathrm{EI}): \mathrm{m} / z(\%) 274\left(\mathrm{M}^{+}\right)$, 147, 120 (100). HRMS calcd. for $\mathrm{C}_{16} \mathrm{H}_{12} \mathrm{O}_{2} \mathrm{~F}_{2}$ : 274.0805; Found: 274.0806 .<smiles>COC(=O)[C@H](Cc1ccc(C(F)(F)F)cc1)NC(=O)OC(C)(C)C</smiles>

(S)-Methyl 2-((tert-butoxycarbonyl)amino)-3-(4-(difluoromethyl)phenyl)propanoate (38). The product (60 mg, 61\% yield) as a white solid (m.p. $70-73{ }^{\circ} \mathrm{C}$ ) was purified with silica gel chromatography (Petroleum ether/EtOAc = 3:1). ${ }^{1} \mathrm{H}$ NMR $\left(400 \mathrm{MHz}, \mathrm{CDCl}_{3}\right) \delta 7.42(\mathrm{~d}, J=8.0 \mathrm{~Hz}, 2$ H), $7.21(\mathrm{~d}, J=8.0 \mathrm{~Hz}, 2 \mathrm{H}), 6.61(\mathrm{t}, J=56.4 \mathrm{~Hz}, 1 \mathrm{H}), 5.01(\mathrm{~d}, J=6.8 \mathrm{~Hz}, 1 \mathrm{H}), 4.61(\mathrm{dd}, J=13.2$ $\mathrm{Hz}, J=6.4 \mathrm{~Hz}, 1 \mathrm{H}), 3.71(\mathrm{~s}, 3 \mathrm{H}), 3.17(\mathrm{dd}, J=13.6 \mathrm{~Hz}, J=5.6 \mathrm{~Hz}, 1 \mathrm{H}), 3.06(\mathrm{dd}, J=13.6 \mathrm{~Hz}, J=$ $5.6 \mathrm{~Hz}, 1 \mathrm{H}), 1.40(\mathrm{~s}, 9 \mathrm{H}) .{ }^{19} \mathrm{~F}$ NMR $\left(376 \mathrm{MHz}, \mathrm{CDCl}_{3}\right) \delta-110.5(\mathrm{~d}, J=56.4 \mathrm{~Hz}, 2 \mathrm{~F}) .{ }^{13} \mathrm{C} \mathrm{NMR}(100$ $\left.\mathrm{MHz}, \mathrm{CDCl}_{3}\right) \delta 172.1,155.0,139.0(\mathrm{t}, J=2.0 \mathrm{~Hz}), 133.2(\mathrm{t}, J=22.1 \mathrm{~Hz}), 129.7,125.8(\mathrm{t}, J=5.9 \mathrm{~Hz})$, $114.6(\mathrm{t}, J=237.1 \mathrm{~Hz}), 80.1,54.3,52.3,38.2,28.3$. IR (thin film) $v_{\max } 3372,2978,1748,1714,1508$ $\mathrm{cm}^{-1}$. MS (EI): $\mathrm{m} / z(\%) 328\left(\mathrm{M}^{+}-\mathrm{H}\right), 212,88,57$ (100). HRMS calcd. for $\mathrm{C}_{16} \mathrm{H}_{21} \mathrm{NO}_{4} \mathrm{~F}_{2}: 329.1439$; Found: 329.1436. 
<smiles>O=C1[C@H](CC[C@@H](O)c2ccc(F)cc2)C(c2ccc(C(F)(F)F)cc2)N1c1ccc(F)cc1</smiles>

(3R)-4-(4-(Difluoromethyl)phenyl)-1-(4-fluorophenyl)-3-((S)-3-(4-fluorophenyl)-3-

hydroxypropyl)azetidin-2-one (39). The product (77 mg, 58\% yield) as a white solid (m.p. 141-146 ${ }^{\circ} \mathrm{C}$ ) was purified with silica gel chromatography (Petroleum ether/EtOAc $\left.=3: 1\right) .{ }^{1} \mathrm{H} \mathrm{NMR}(400 \mathrm{MHz}$, $\left.\mathrm{CDCl}_{3}\right) \delta 7.52(\mathrm{~d}, J=7.6 \mathrm{~Hz}, 2 \mathrm{H}), 7.41(\mathrm{~d}, J=7.6 \mathrm{~Hz}, 2 \mathrm{H}), 7.31-7.28(\mathrm{~m}, 2 \mathrm{H}), 7.23-7.19(\mathrm{~m}, 2 \mathrm{H})$, $7.02(\mathrm{t}, J=8.4 \mathrm{~Hz}, 2 \mathrm{H}), 6.94(\mathrm{t}, J=8.4 \mathrm{~Hz}, 2 \mathrm{H}), 6.64(\mathrm{t}, J=56.4 \mathrm{~Hz}, 1 \mathrm{H}), 4.72(\mathrm{~m}, 1 \mathrm{H}), 4.67$ (d, $J$ $=2.4 \mathrm{~Hz}, 1 \mathrm{H}), 3.08(\mathrm{td}, J=6.8 \mathrm{~Hz}, J=2.4 \mathrm{~Hz}, 1 \mathrm{H}), 2.11(\mathrm{~d}, J=3.6 \mathrm{~Hz}, 1 \mathrm{H}), 2.04-1.90(\mathrm{~m}, 4 \mathrm{H}) .{ }^{19} \mathrm{~F}$ NMR $\left(376 \mathrm{MHz}, \mathrm{CDCl}_{3}\right) \delta-110.9(\mathrm{~d}, J=56.4 \mathrm{~Hz}, 2 \mathrm{~F}),-114.7(\mathrm{~m}, 1 \mathrm{~F}),-117.6(\mathrm{~m}, 1 \mathrm{~F}) .{ }^{13} \mathrm{C} \mathrm{NMR}$ $\left(100 \mathrm{MHz} \mathrm{CDCl}_{3}\right) \delta 167.0,162.2(\mathrm{~d}, J=244.3 \mathrm{~Hz}), 159.1(\mathrm{~d}, J=242.5 \mathrm{~Hz}), 140.4(\mathrm{t}, J=2.0 \mathrm{~Hz})$, $139.9(\mathrm{~d}, J=3.1 \mathrm{~Hz}), 134.8(\mathrm{t}, J=22.5 \mathrm{~Hz}), 133.6(\mathrm{~d}, J=2.3 \mathrm{~Hz}), 127.3(\mathrm{~d}, J=8.0 \mathrm{~Hz}), 126.6(\mathrm{t}, J=$ $6.0 \mathrm{~Hz}), 126.2,118.3(\mathrm{~d}, J=7.8 \mathrm{~Hz}), 115.9(\mathrm{~d}, J=22.8 \mathrm{~Hz}), 115.4(\mathrm{~d}, J=20.1 \mathrm{~Hz}), 114.2(\mathrm{t}, J=237.7$ $\mathrm{Hz}), 73.2,60.9,60.5,36.6,25.1$. IR (thin film) $v_{\max } 3432,2928,1739,1714,1507 \mathrm{~cm}^{-1}$. MS (EI): $\mathrm{m} / z$ (\%) $443\left(\mathrm{M}^{+}\right), 333,292,250$ (100). HRMS calcd. for $\mathrm{C}_{25} \mathrm{H}_{21} \mathrm{NO}_{2} \mathrm{~F}_{4}$ : 443.1508; Found: 443.1512.

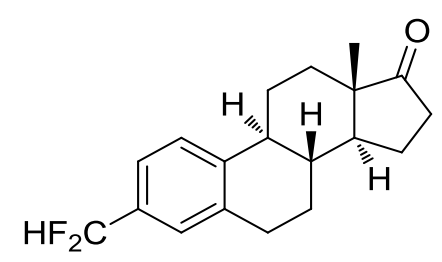

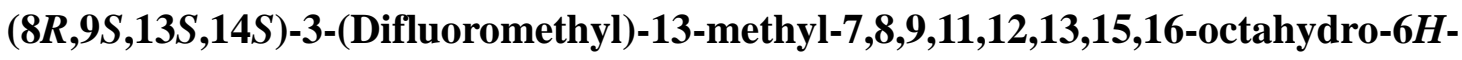

cyclopenta[ $[\boldsymbol{a}]$ phenanthren-17(14H)-one (40). The product (69 mg, 76\% yield) as a white solid (m.p. $59-62{ }^{\circ} \mathrm{C}$ ) was purified with silica gel chromatography (Petroleum ether/EtOAc $\left.=3: 1\right) .{ }^{1} \mathrm{H}$ NMR $(400$ $\left.\mathrm{MHz} \mathrm{CDCl}_{3}\right) \delta 7.37(\mathrm{~d}, J=8.4 \mathrm{~Hz}, 1 \mathrm{H}), 7.27(\mathrm{~d}, J=8.4 \mathrm{~Hz}, 1 \mathrm{H}), 7.24(\mathrm{~s}, 1 \mathrm{H}), 6.58(\mathrm{t}, J=56.4 \mathrm{~Hz}$, $1 \mathrm{H}), 2.97-2.94(\mathrm{~m}, 2 \mathrm{H}), 2.51(\mathrm{q}, J=8.8 \mathrm{~Hz}, 1 \mathrm{H}), 2.47-2.42(\mathrm{~m}, 1 \mathrm{H}), 2.32(\mathrm{t}, J=10.0 \mathrm{~Hz}, 1 \mathrm{H}), 2.19$ 2.12 (m, 1 H), 2.10-2.03 (m, 2 H), 1.99-1.96 (m, 1 H), 1.67-1.42 (m, 6 H), 0.91 (s, 3 H). ${ }^{19}$ F NMR (376 $\left.\mathrm{MHz}, \mathrm{CDCl}_{3}\right) \delta-109.9(\mathrm{dd}, J=56.4 \mathrm{~Hz}, J=5.3 \mathrm{~Hz}, 2 \mathrm{~F}) .{ }^{13} \mathrm{C} \mathrm{NMR}\left(125.7 \mathrm{MHz}, \mathrm{CDCl}_{3}\right) \delta 220.6$, $142.6(\mathrm{t}, J=1.9 \mathrm{~Hz}), 137.1,131.9(\mathrm{t}, J=22.4 \mathrm{~Hz}), 126.1(\mathrm{t}, J=5.9 \mathrm{~Hz}), 125.7,122.8(\mathrm{t}, J=16.6 \mathrm{~Hz})$, $114.9(\mathrm{t}, J=237.9 \mathrm{~Hz}), 50.5,47.9,44.4,37.9,35.8,31.5,29.3,26.2,25.6,21.5,13.8 . \mathrm{R}$ (thin film) 
$v_{\max }$ 2984, 1734, $1616 \mathrm{~cm}^{-1}$. MS (EI): $\mathrm{m} / \mathrm{z}(\%) 304\left(\mathrm{M}^{+}\right), 304$ (100). HRMS calcd. for $\mathrm{C}_{19} \mathrm{H}_{22} \mathrm{OF}_{2}$ : 304.1639; Found: 304.1634.

\section{References:}

1) Mehta, V. P. \& Greaney, M. F. S-, N-, and Se-Difluoromethylation using sodium chlorodifluoroacetate. Org. Lett, 15, 5036-5039 (2013).

2) Grushin, V. V. \& Marshall, W. J. Facile Ar-CF 3 Bond Formation at Pd. Strikingly Different Outcomes of Reductive Elimination from $\left[\left(\mathrm{Ph}_{3} \mathrm{P}\right)_{2} \mathrm{Pd}\left(\mathrm{CF}_{3}\right) \mathrm{Ph}\right]$ and $\left[(\mathrm{Xantphos}) \mathrm{Pd}\left(\mathrm{CF}_{3}\right) \mathrm{Ph}\right] . J . A m$. Chem. Soc. 128, 12644-12645 (2006).

3) Singh, R.P. \& Shreeve, J. M. Concentration-dependent reactions of deoxofluor with arylglyoxal hydrates: a new route to polyfluoro ethers. Org.Lett. 3, 2713-2715 (2001).

4) Moulder, J. F.; Stickle,W. F.; Sobol, P. E. \& Bomben,K. D. Handbook of X-Ray Photoelectron Spectroscopy: A Reference Book of Standard Spectra for Identification and Interpretation of XPS Data, Perkin-Elmer, Eden Prairie, Minn, USA (1992).

5) Fier, P. S. \& Hartwig, J. F. Copper-mediated difluoromethylation of aryl and vinyl iodides. J. Am. Chem. Soc. 134, 5524-5527 (2012).

6) Ge, S.; Chaładaj, W. \& Hartwig, J. F. Pd-catalyzed $\alpha$-arylation of $\alpha, \alpha$-difluoroketones with aryl bromides and chlorides. A route to difluoromethylarenes. J. Am. Chem. Soc. 136, 4149-4152 (2014).

7) Prakash, G. K. S.; Ganesh, S. K.; Jones, J.-P.; Kulkarni, A.; Masood, K.; Swabeck, J. K. \& Olah, G. A. Copper - Mediated Difluoromethylation of (Hetero) aryl Iodides and $\beta$-Styryl Halides with Tributyl (difluoromethyl) stannane. Angew. Chem., Int. Ed. 51, 12090-12094 (2012).

8) Fujikawa, K.; Fujioka, Y.; Kobayashi, A. \& Amii, H. A new method for aromatic difluoromethylation: copper-catalyzed cross-coupling and decarboxylation sequence from aryl iodides. Org. Lett. 13, 5560-5563 (2011).

9) Rowbotham, J. B.; Janzen, A. F.; Peeling, J. \& Schaefer, T. Proton Magnetic Resonance Studies of Rotational Isomerism in Halotoluenes. X. Estimates of Conformational Preferences and Rotational Barriers in Benzal Fluoride and Some Dichloro Derivatives. Can. J. Chem. 52, 481-488 (1974).

10) Sharma, R.; Halder, S. \& Kumar, S. PCT Int. Appl. WO 2014170842 (2014).

11) Kori. M.; Hamamura. M.; Fuse. H. \& Yamamoto.T. PCT Int.Appl. WO 2002059077 (2002). 
Compound $\mathrm{BrCF}_{2} \mathrm{CO}_{2} \mathrm{~K} \mathrm{~V}$ (determined from a mixture of $\mathrm{V}$ and hydroquinone)
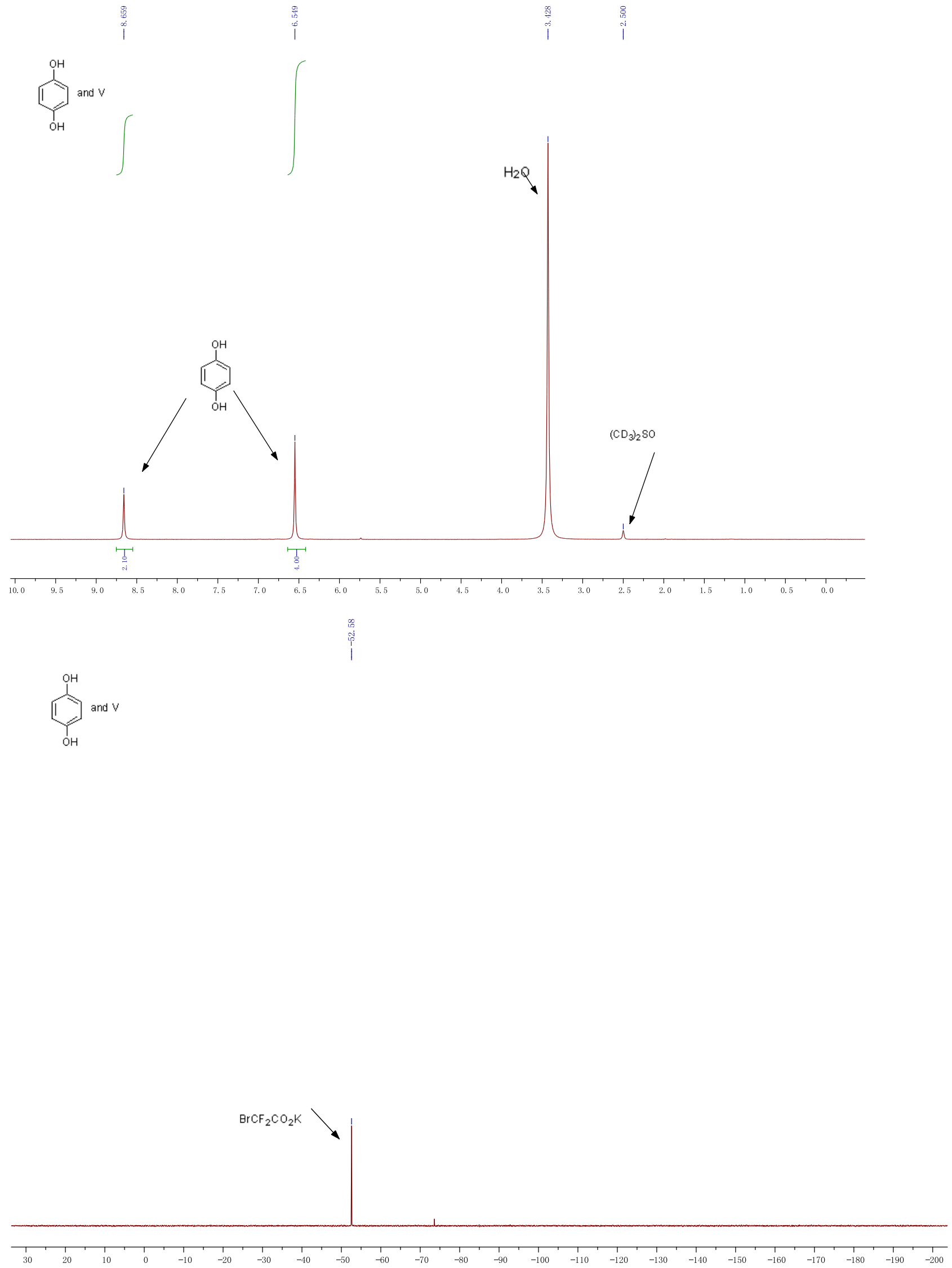


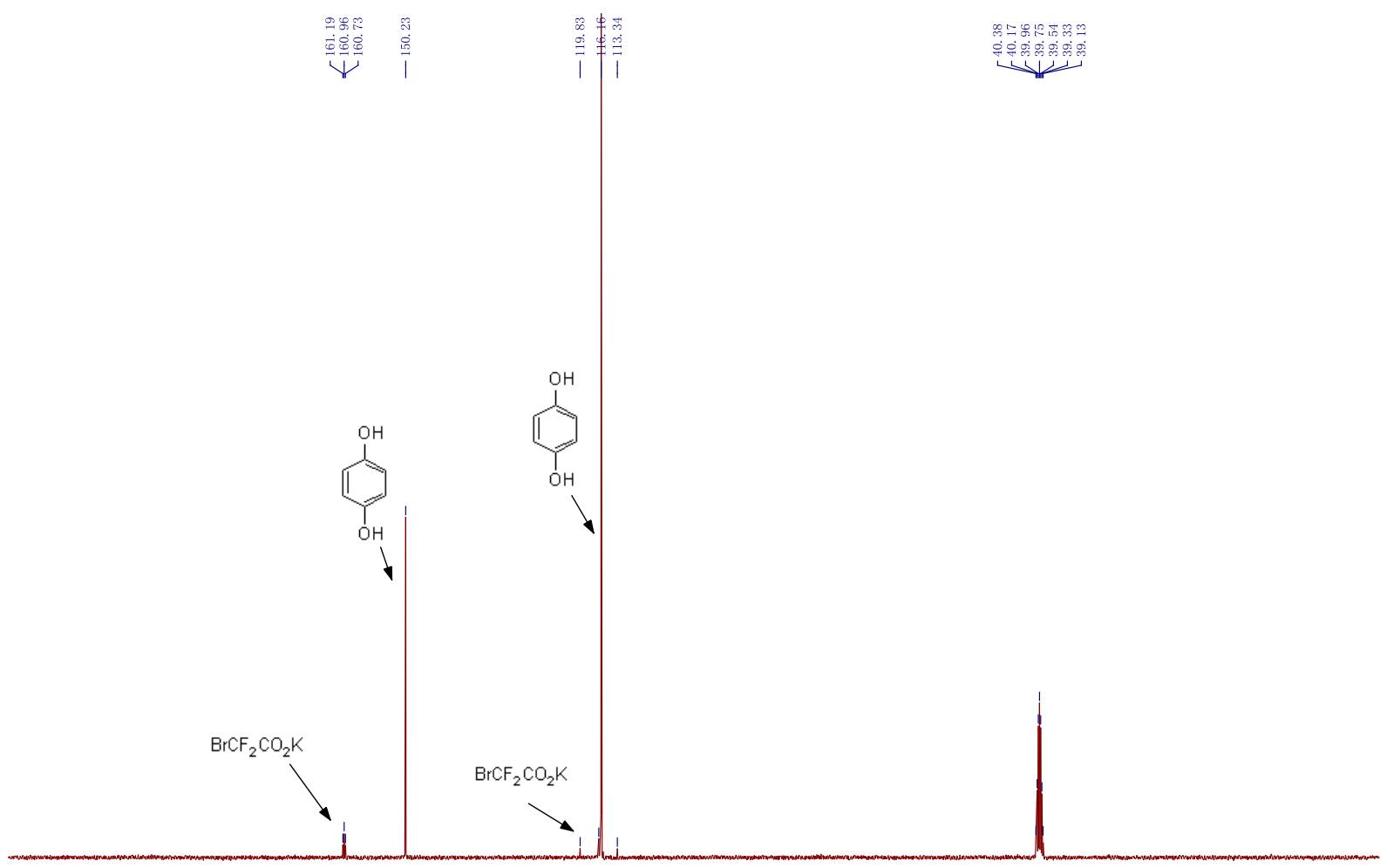

4-(1,1,2,2-Tetrafluoroethyl)-1,1'-biphenyl (3a)

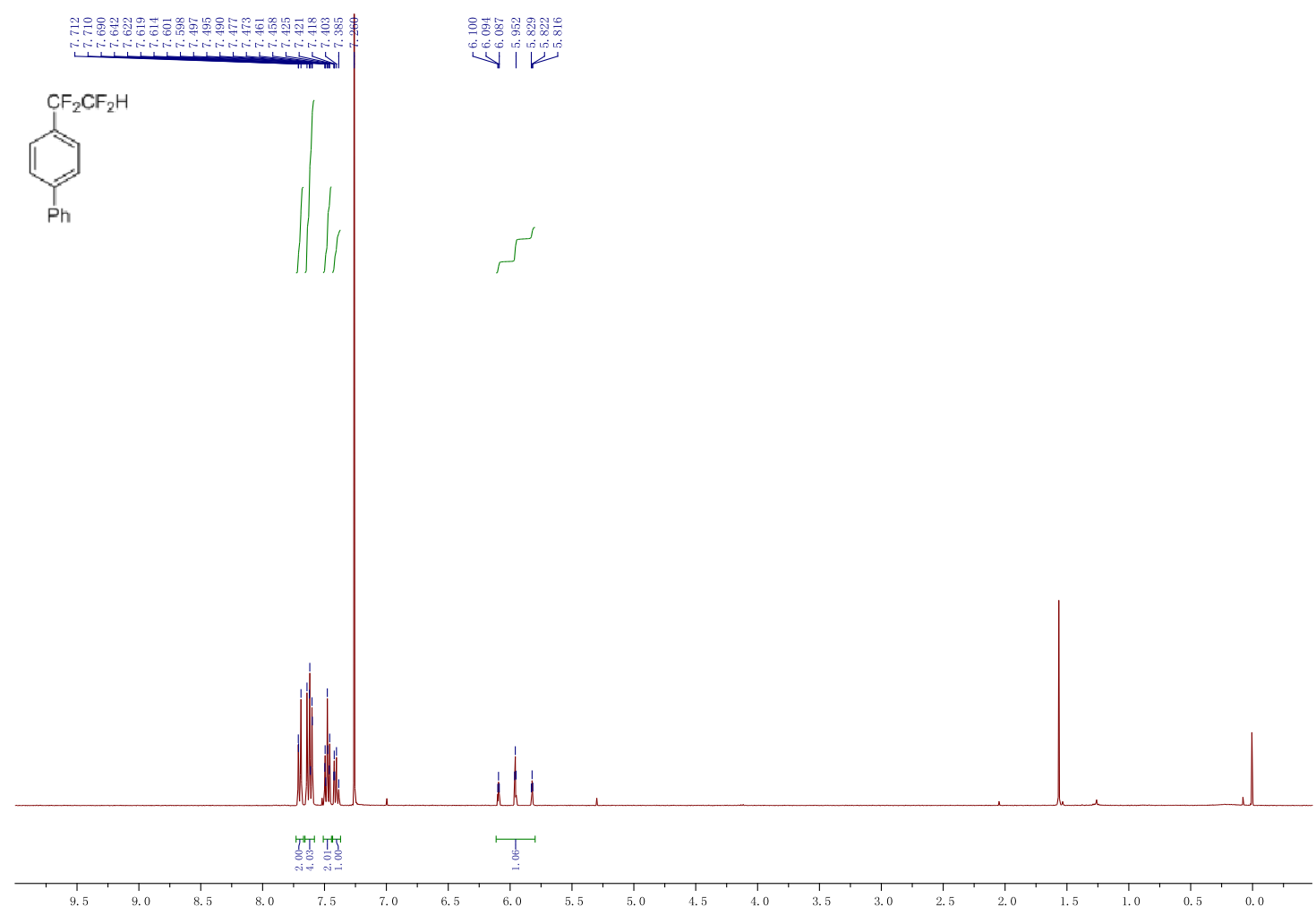



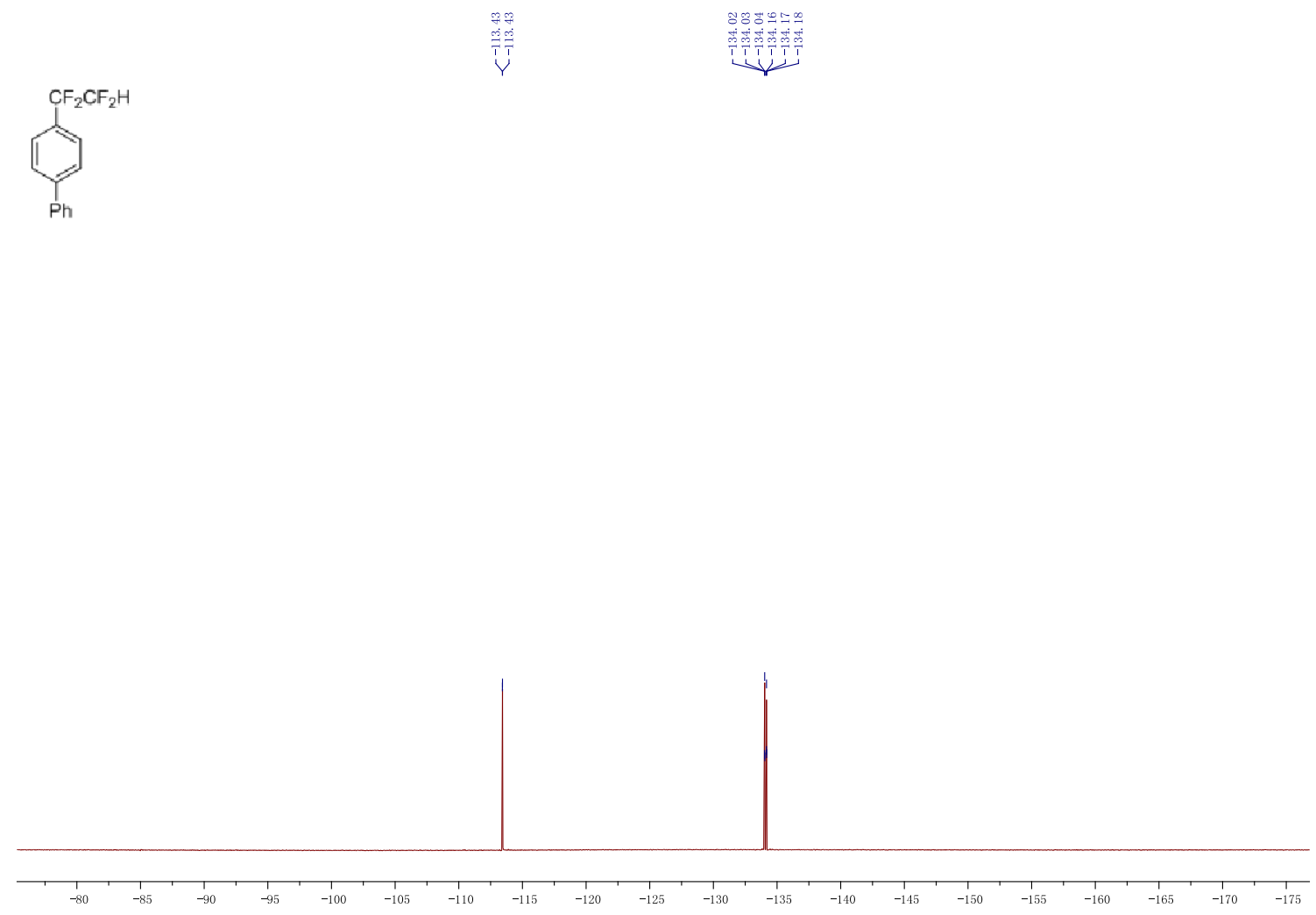
4-(Difluoromethyl)-1,1'-biphenyl (3)

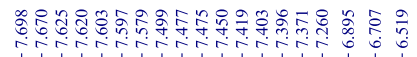
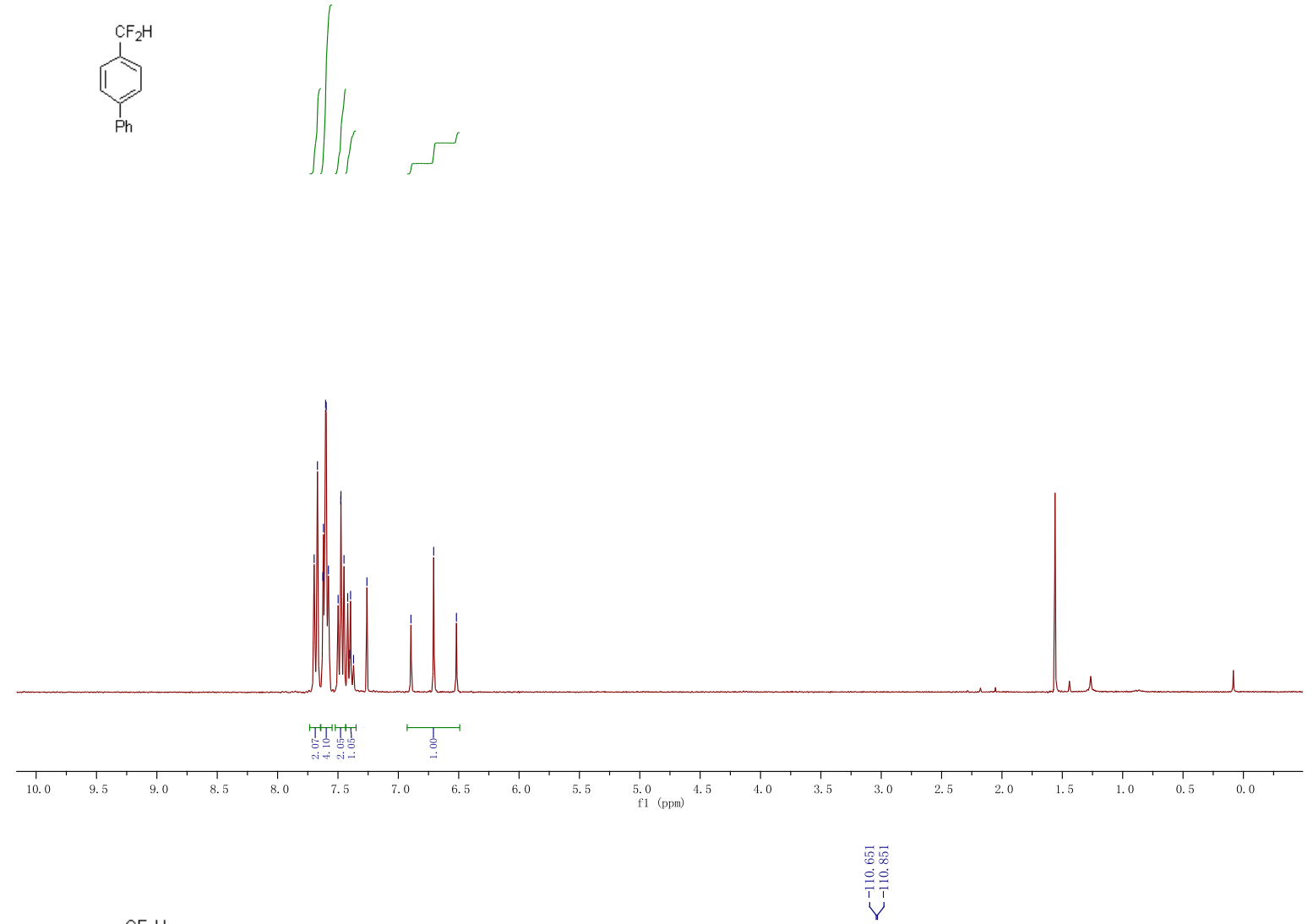

Ph

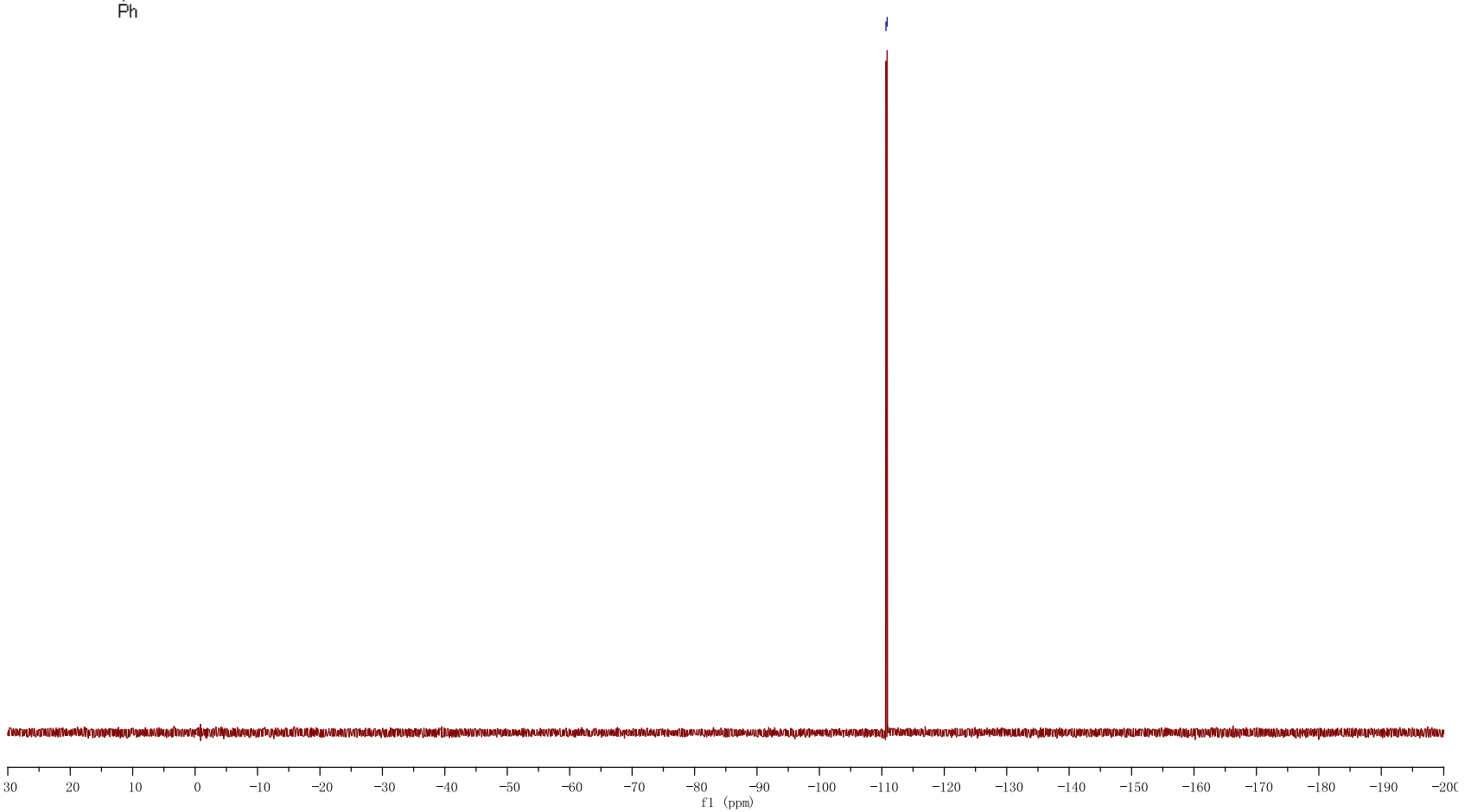


Ph

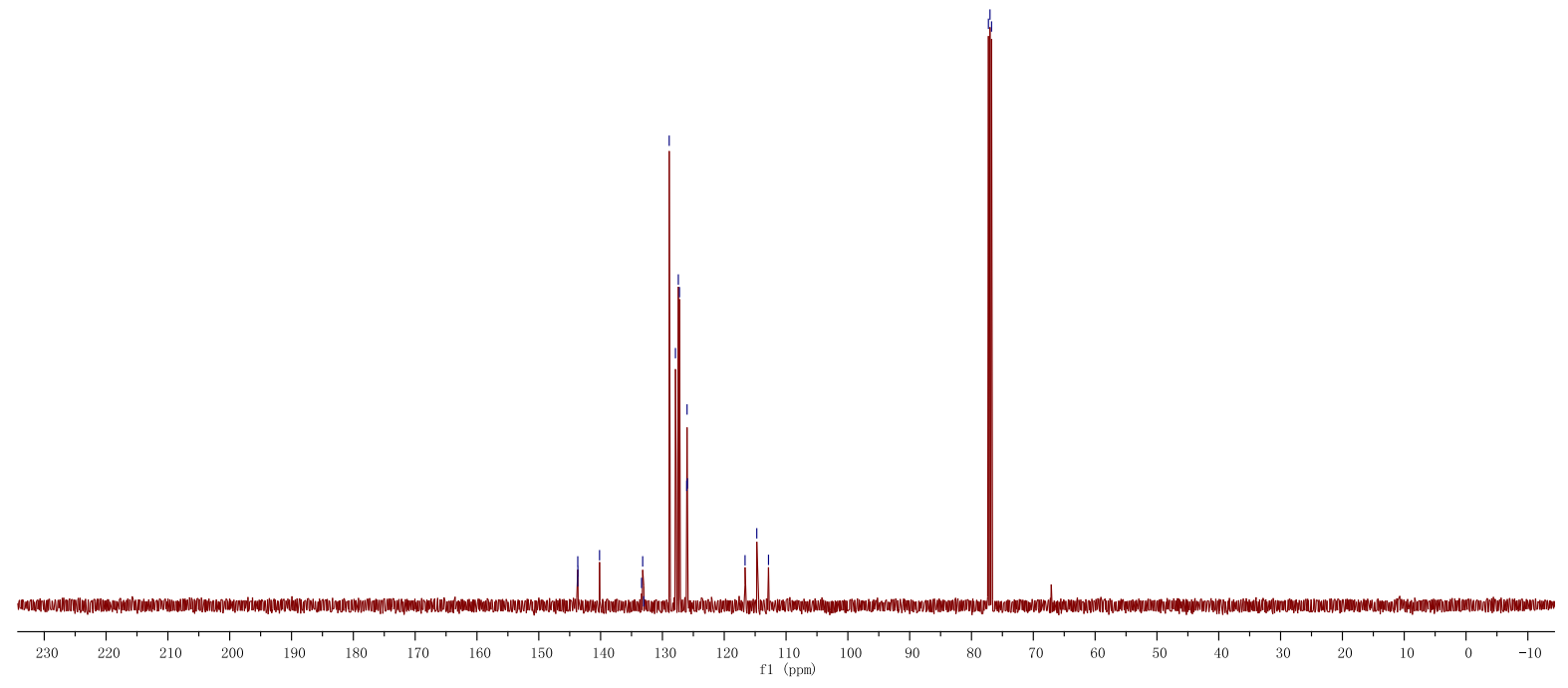

2-(Difluoromethyl)-1,1'-biphenyl (5).
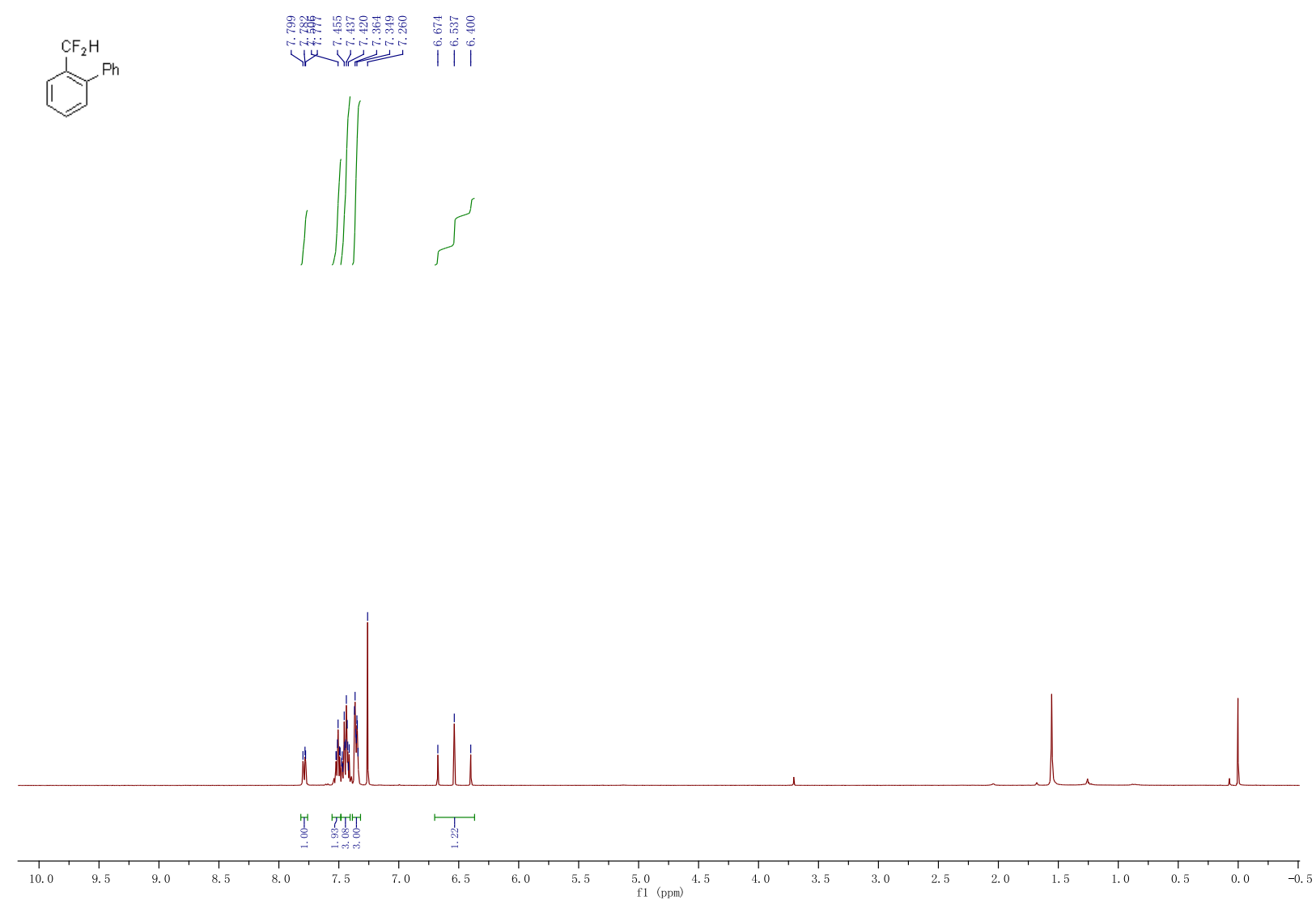
$\mathrm{CF}_{2} \mathrm{H}$

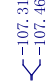
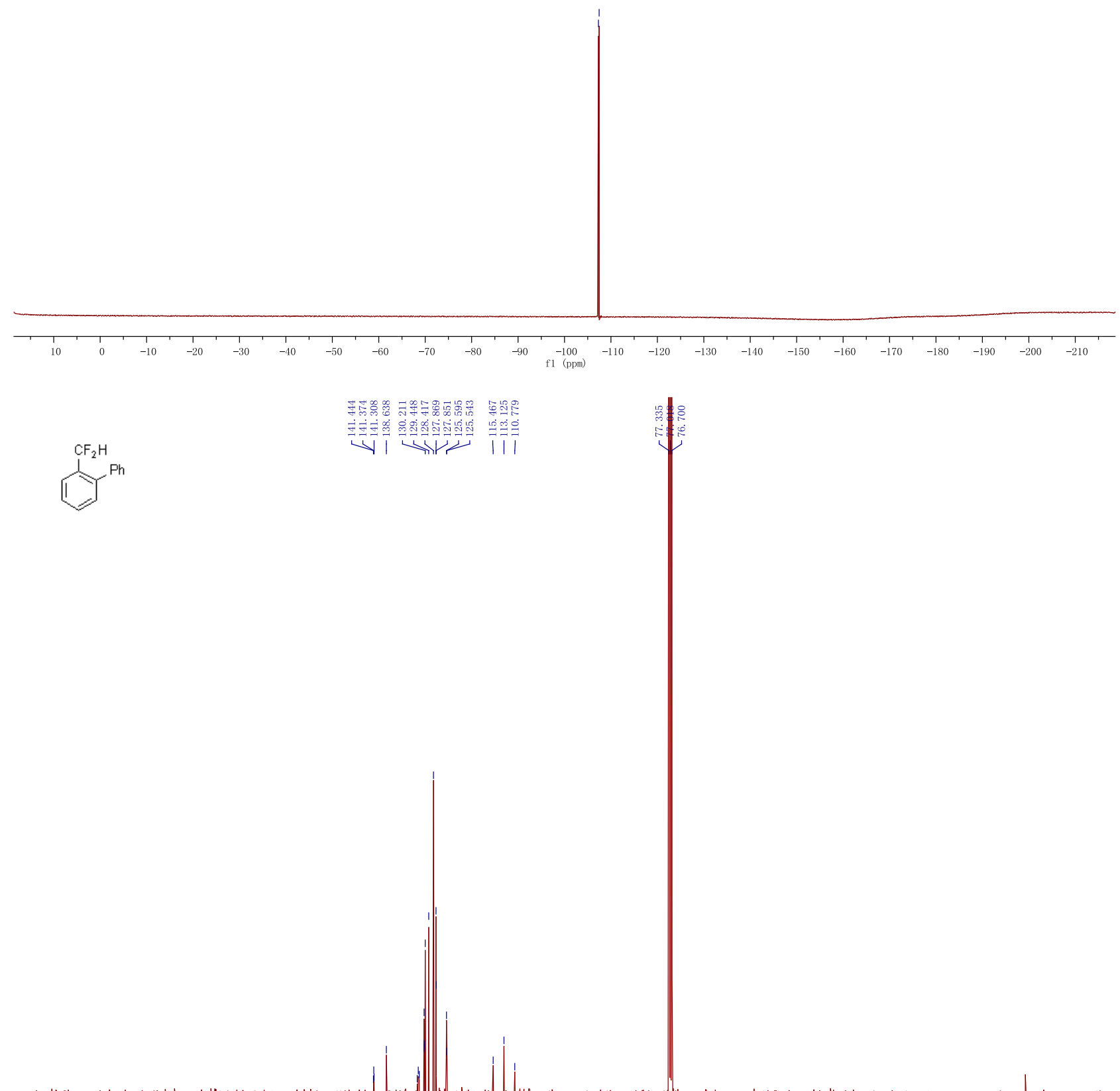

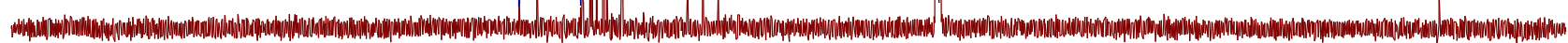


3-(Difluoromethyl)-1,1'-biphenyl (6).
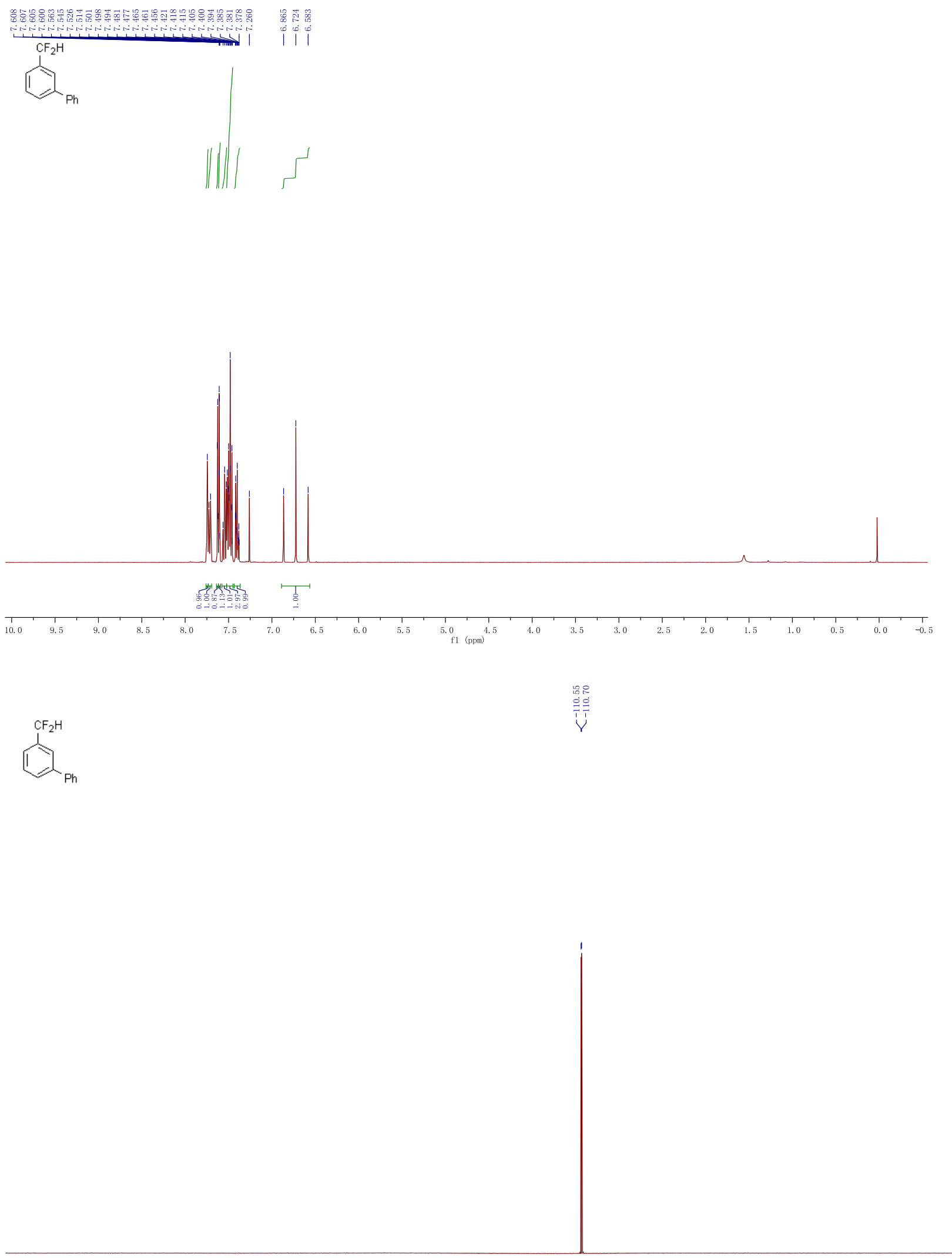

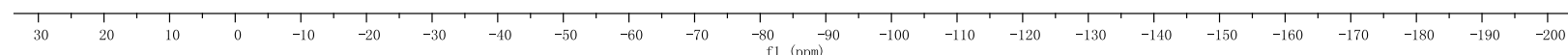




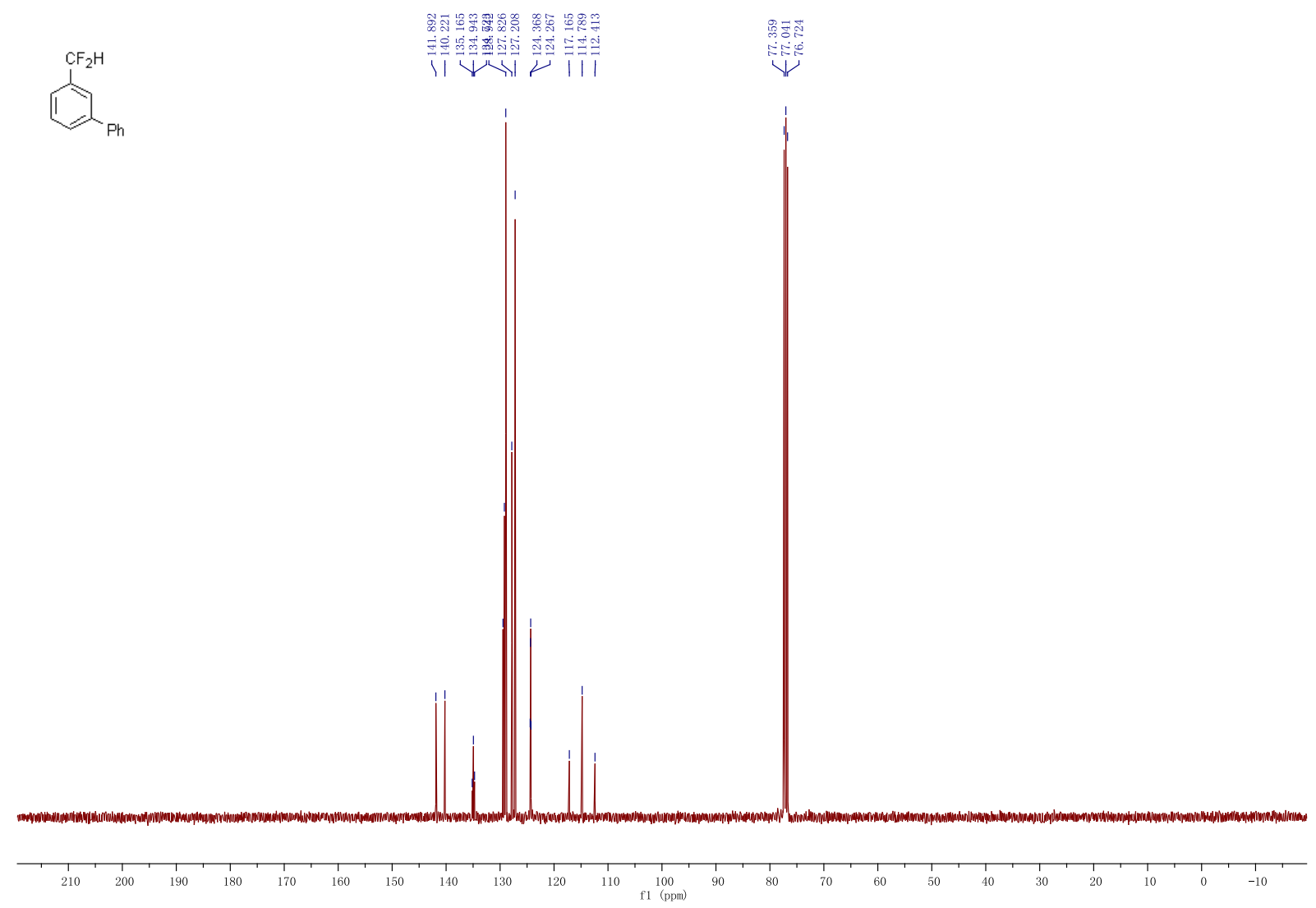

1-(Tert-butyl)-4-(difluoromethyl)benzene (7)
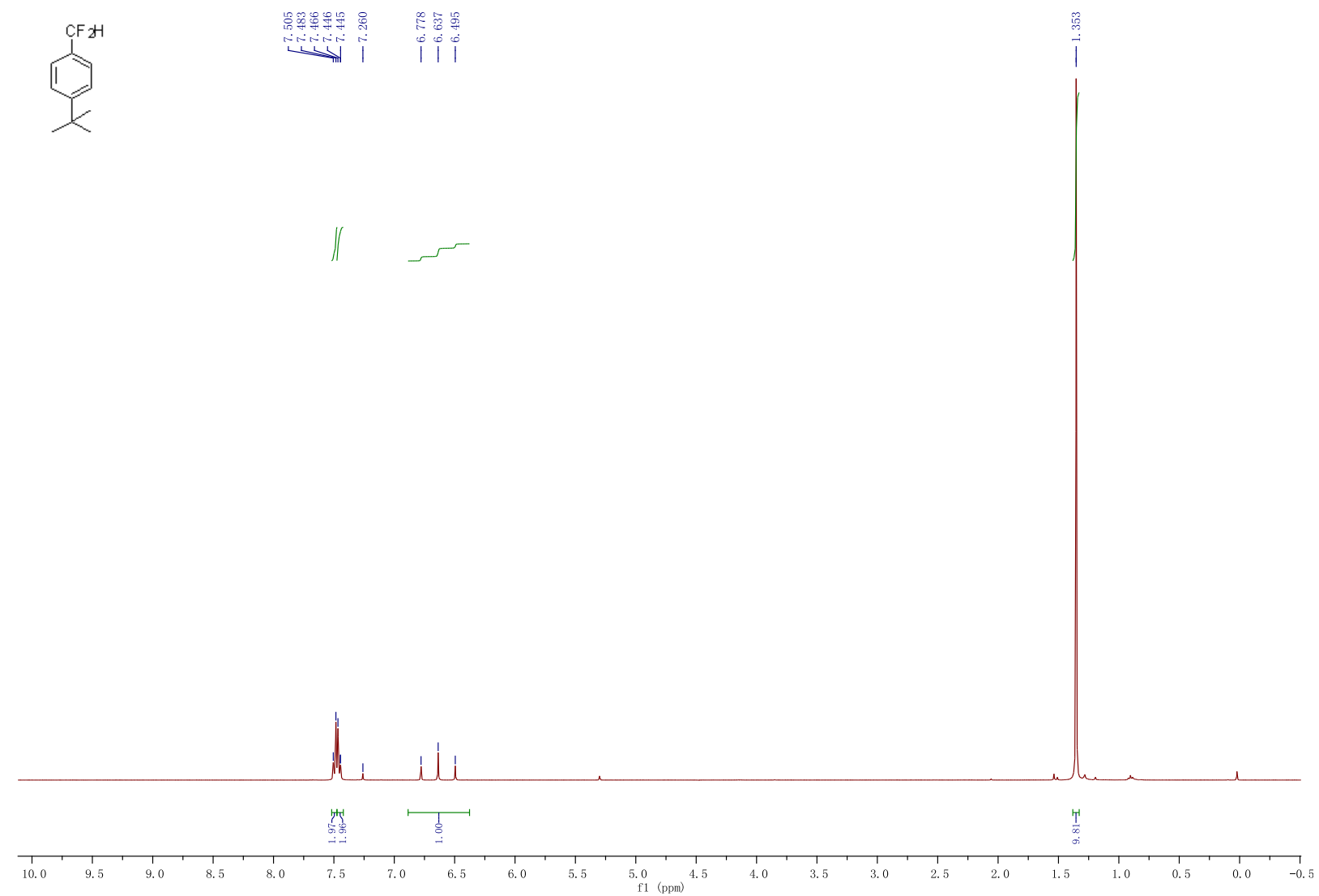


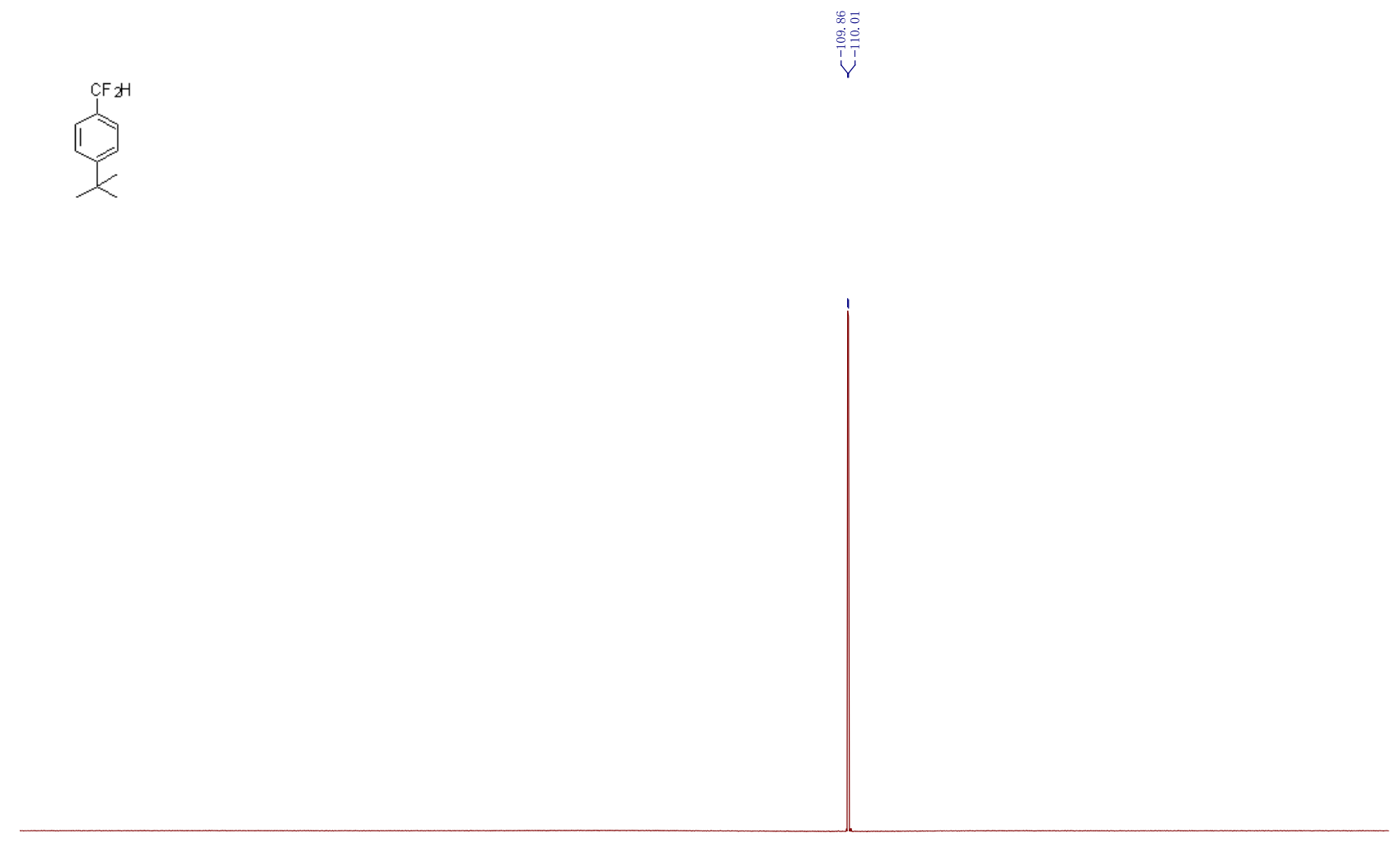

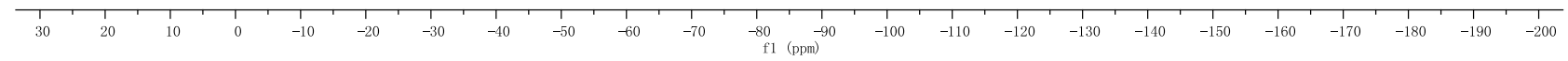

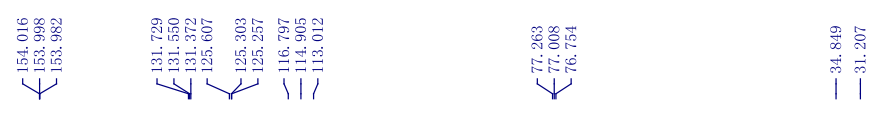

CC2H

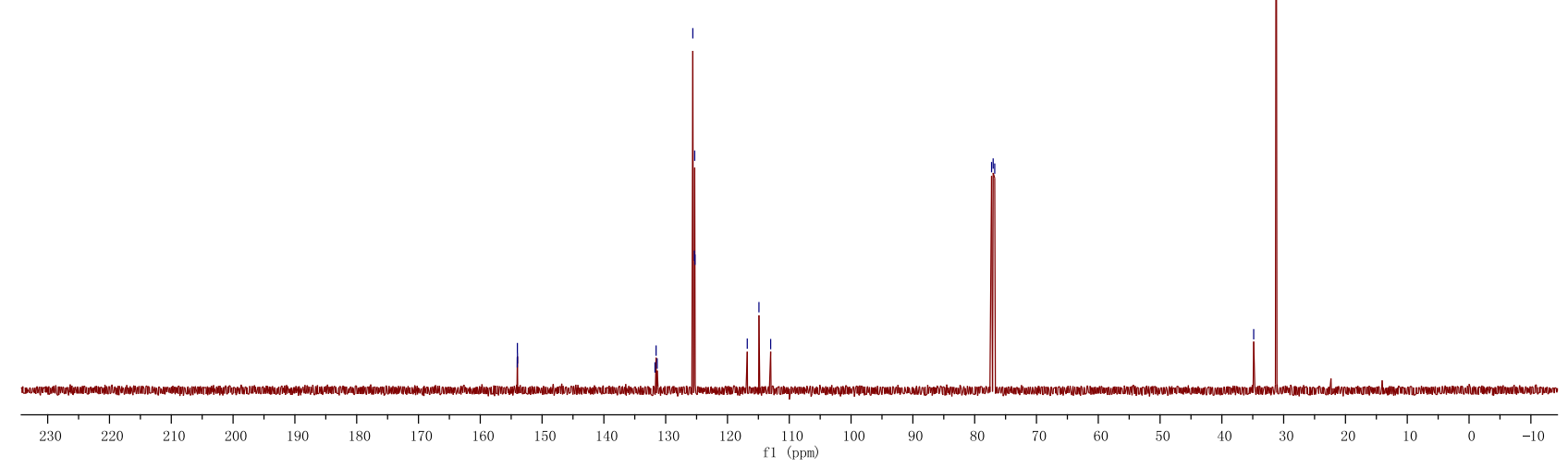


1-(Benzyloxy)-4-(difluoromethyl)benzene (8).
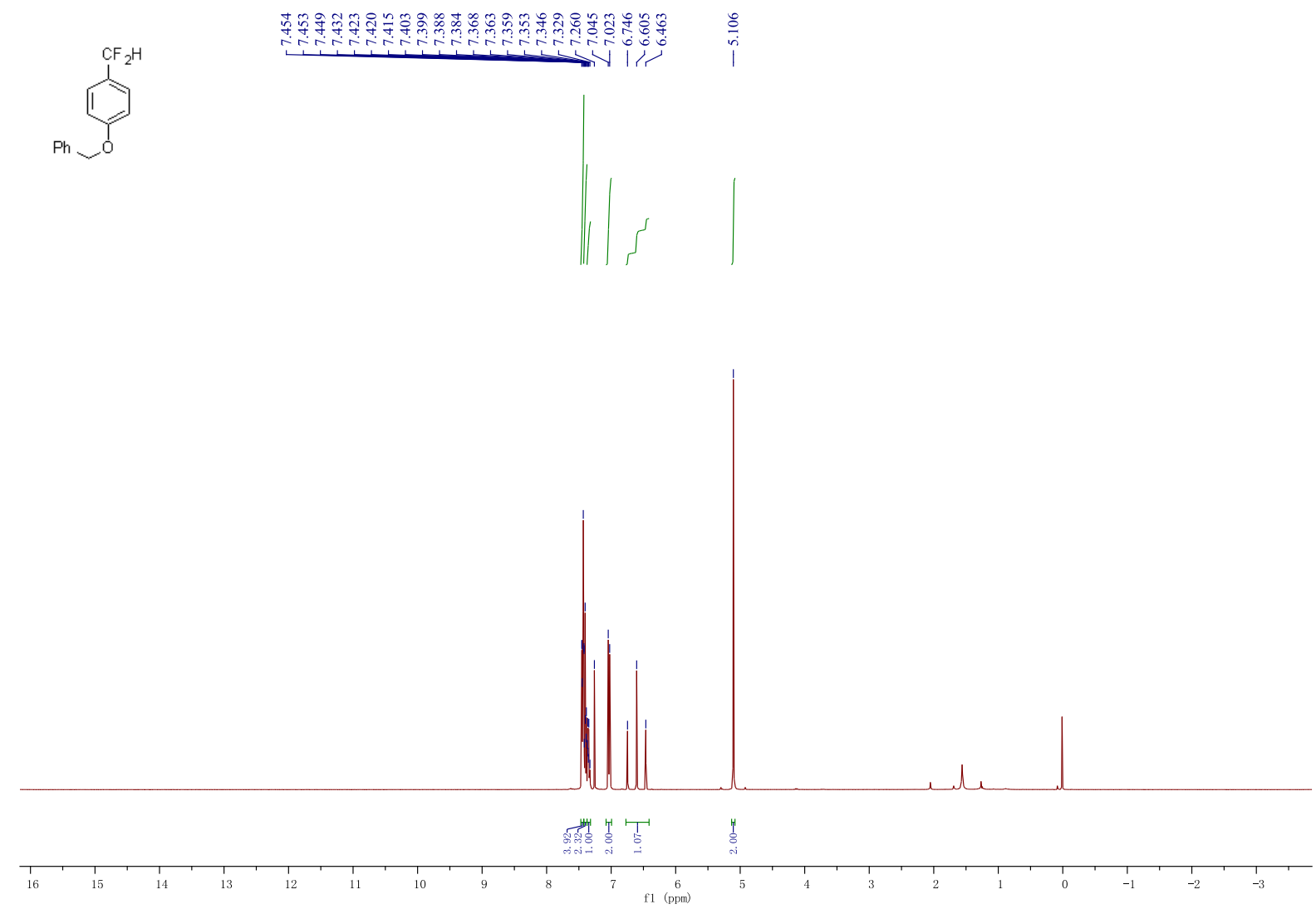<smiles>CCCOc1ccc(CC)cc1</smiles>

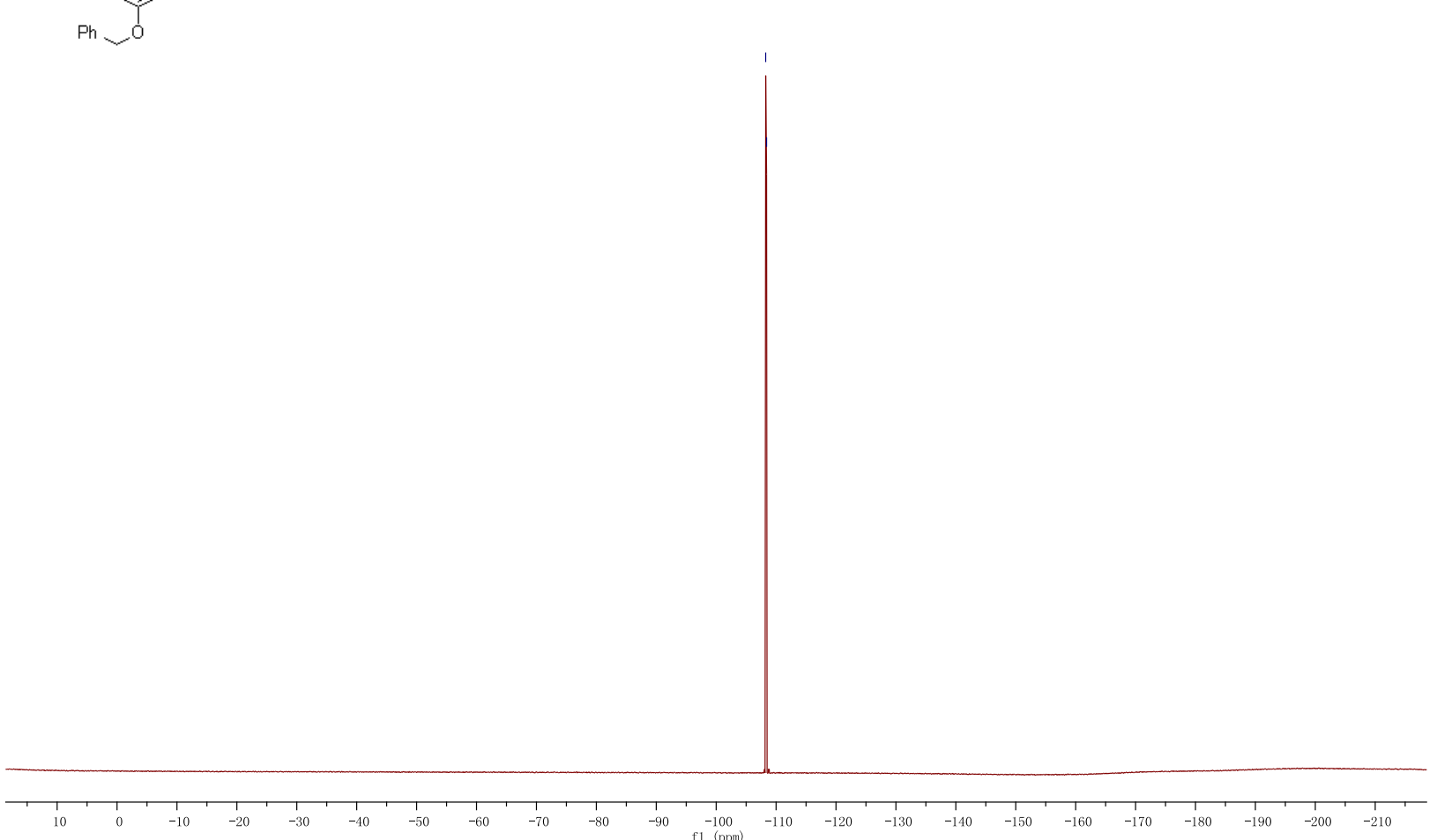




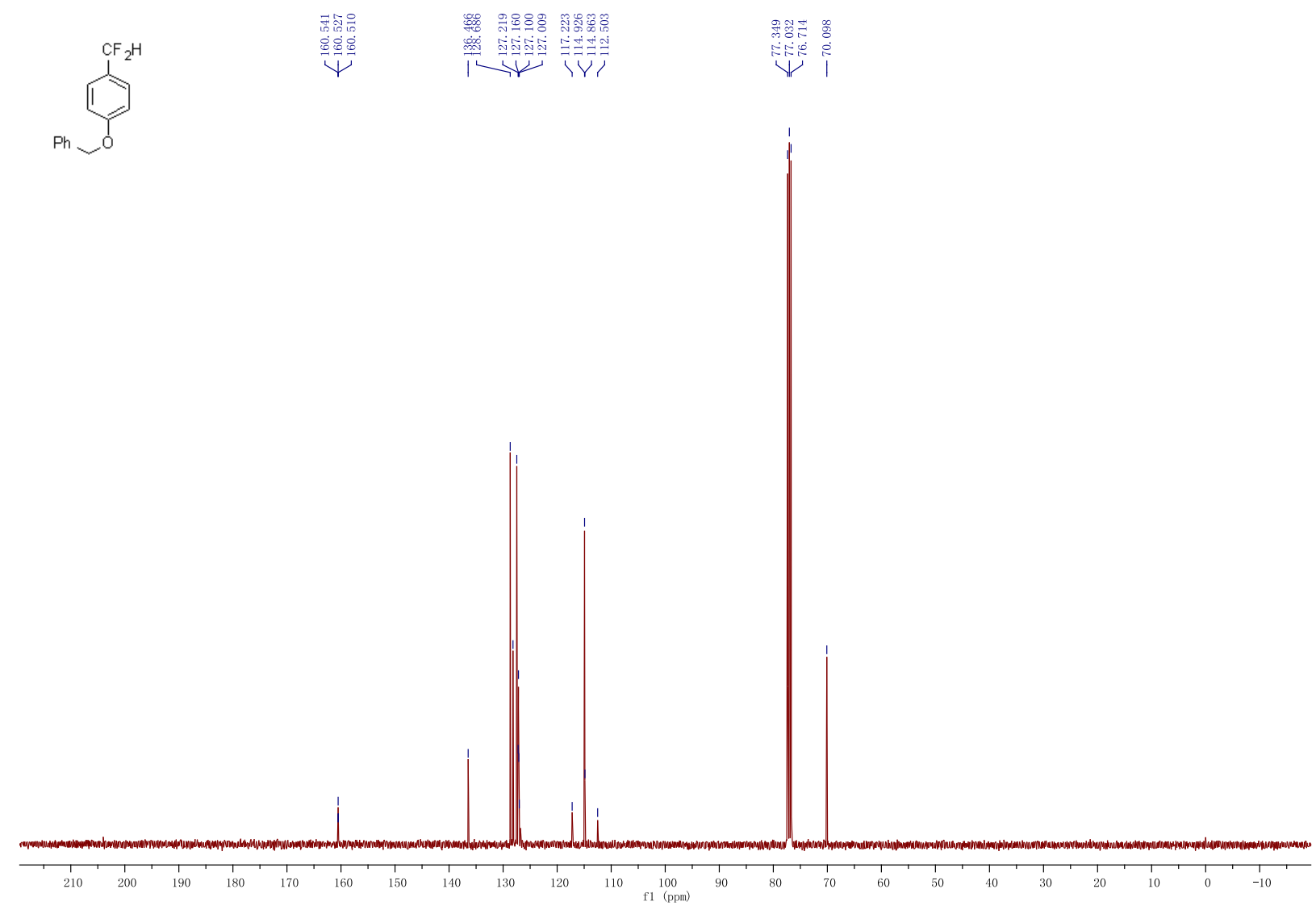

1-(Difluoromethyl)-4-phenoxybenzene (9).
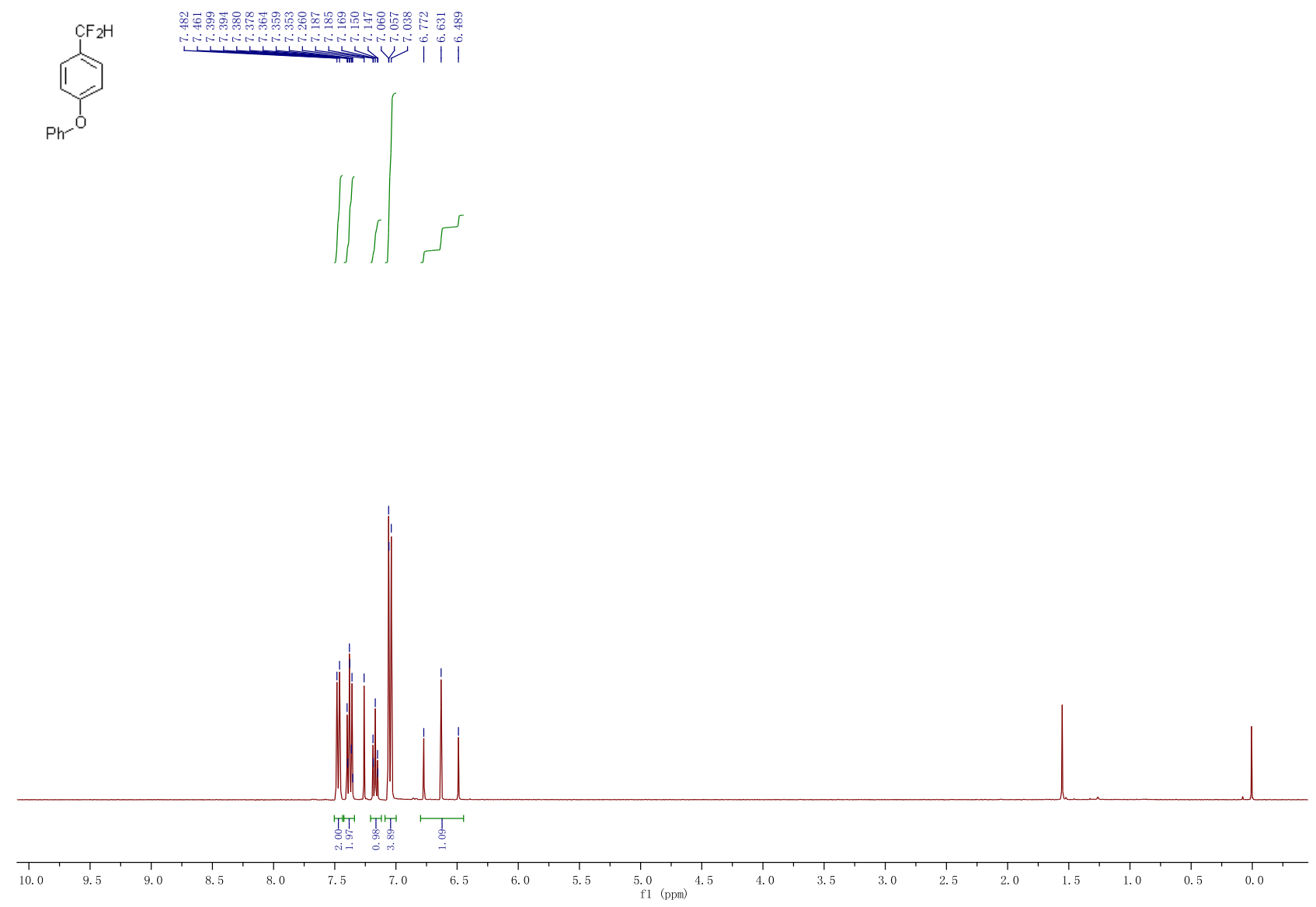

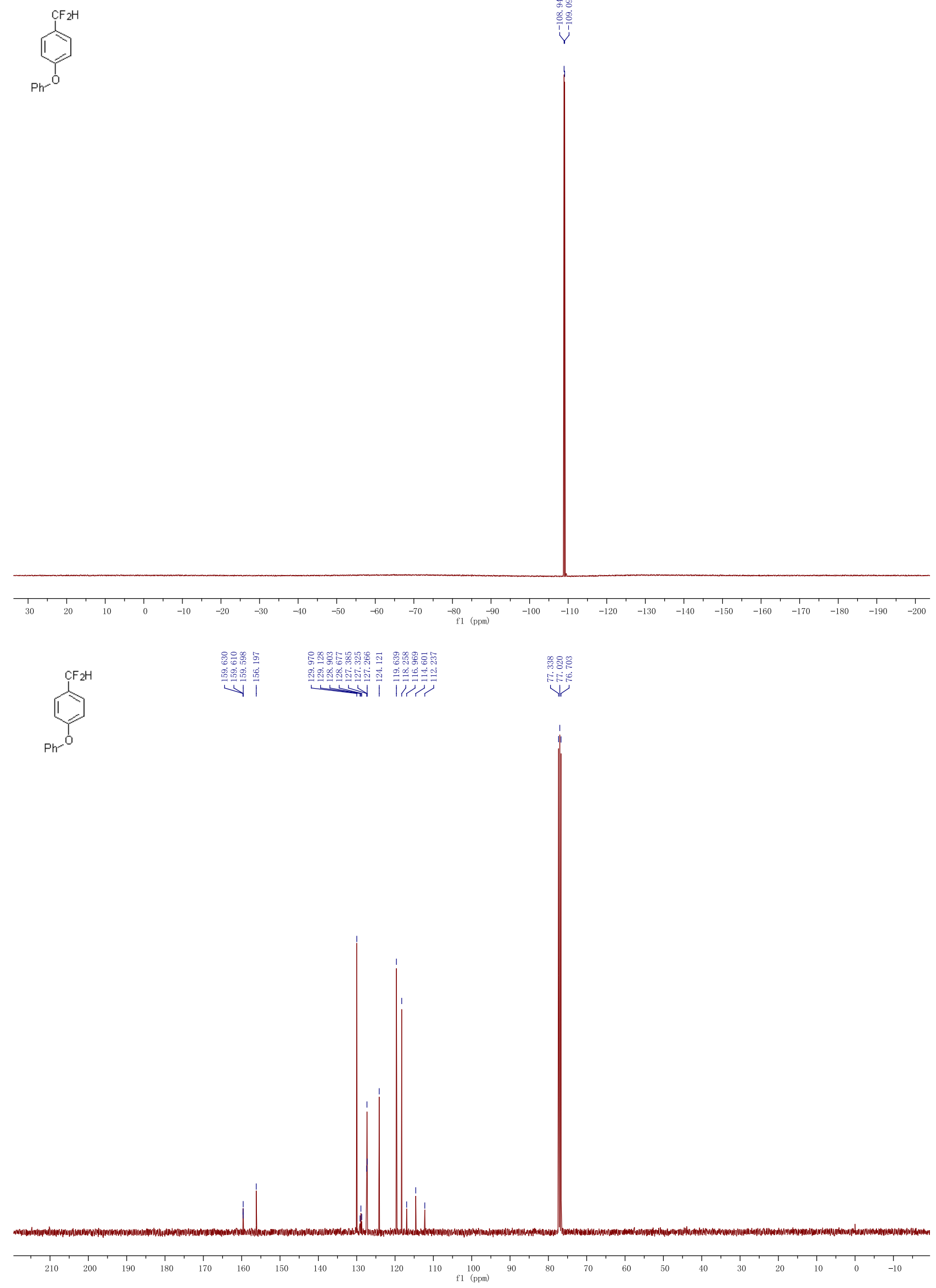
1-(Difluoromethyl)-4-methoxybenzene (10).
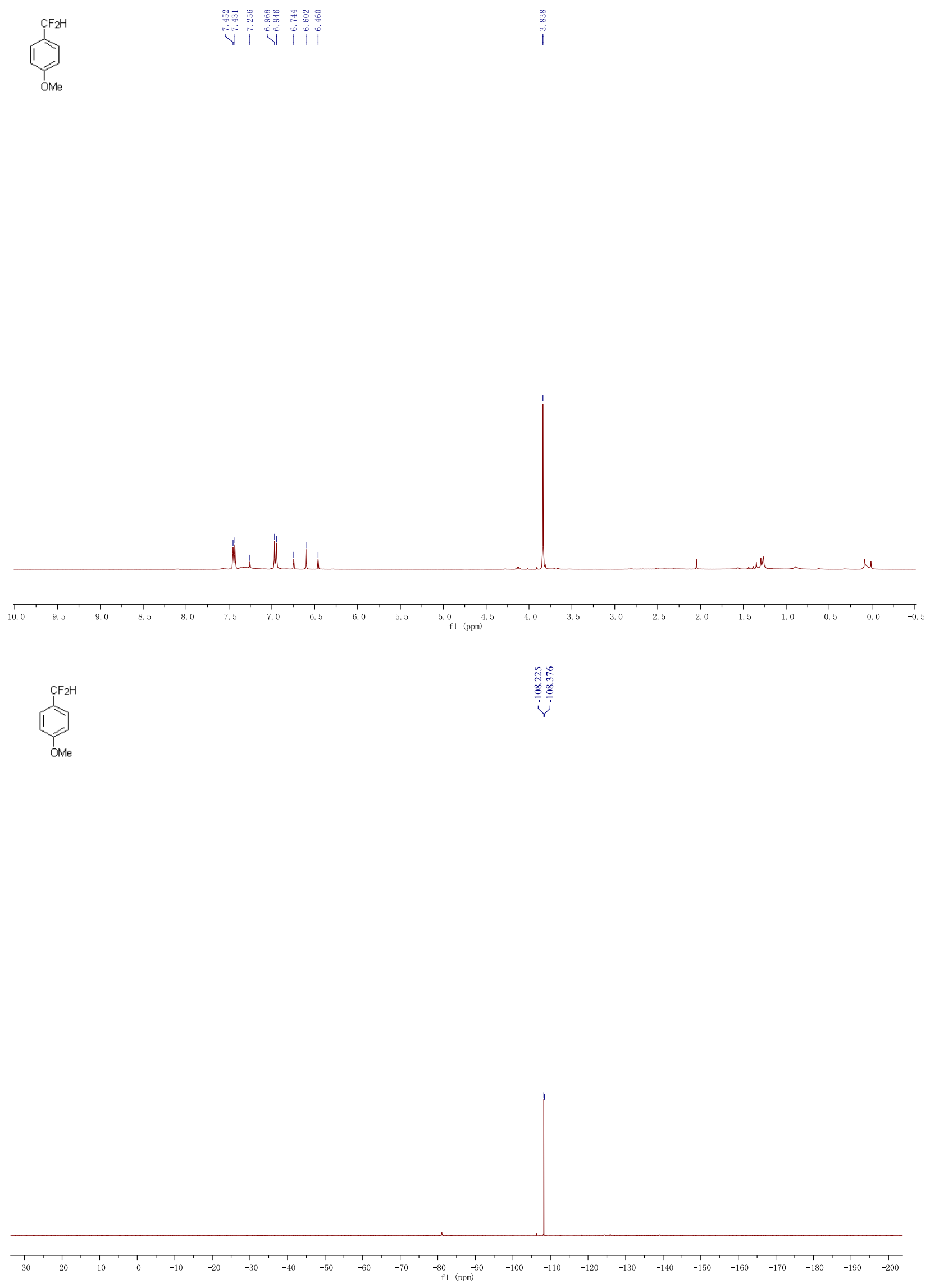

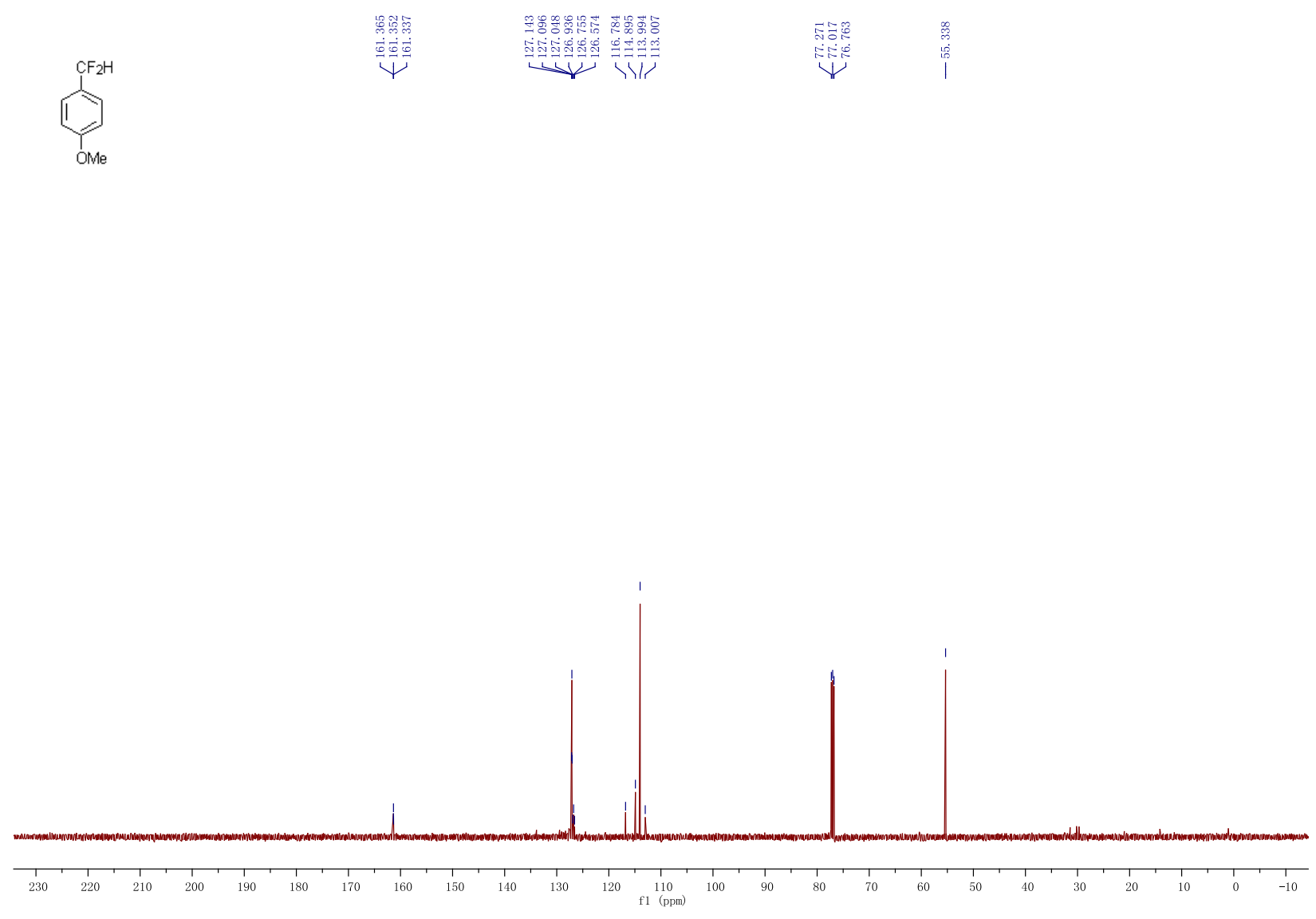

1-(Difluoromethyl)-3,5-dimethoxybenzene (11).

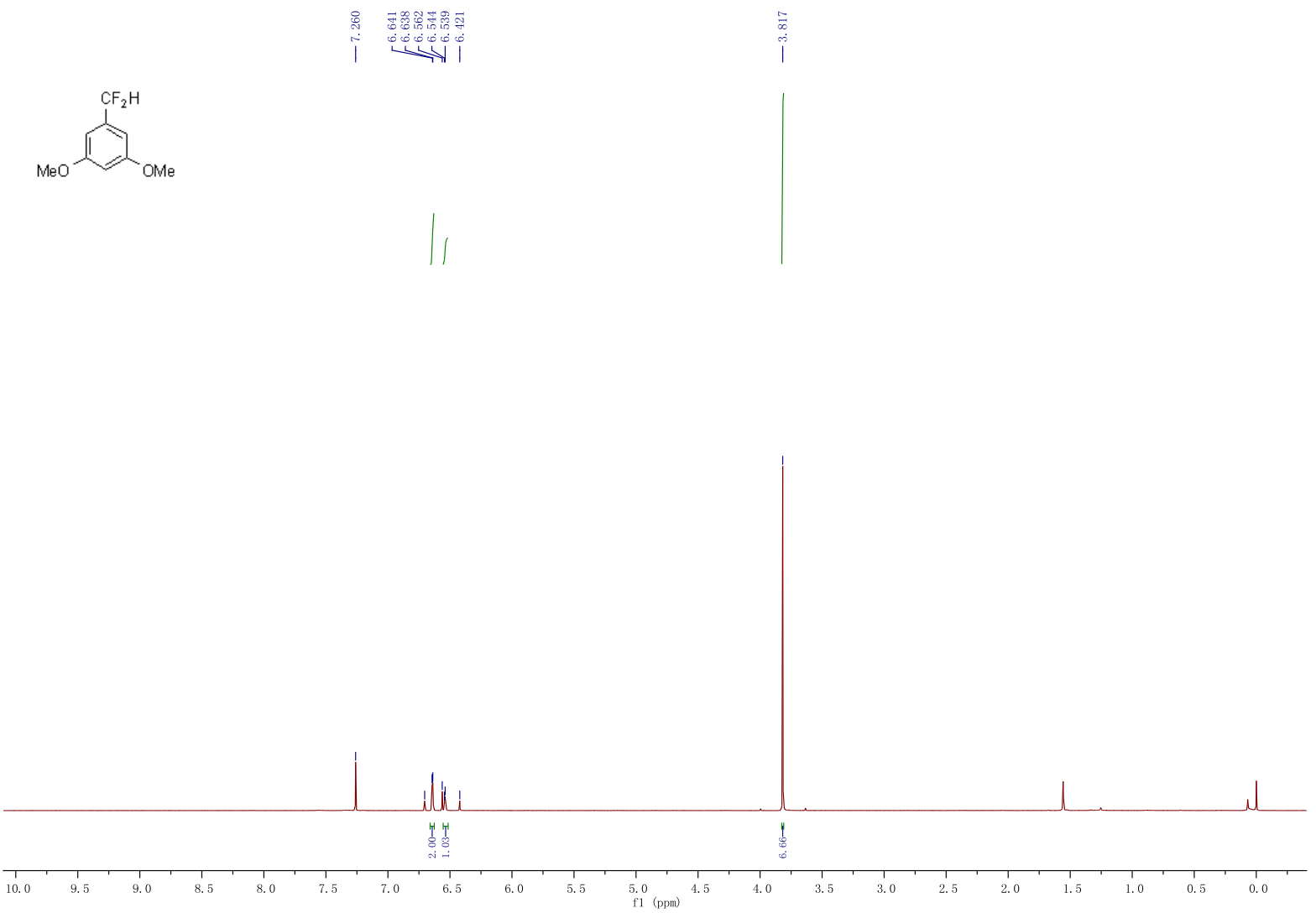


<smiles>COc1cc(OC)cc(C(F)(F)F)c1</smiles>
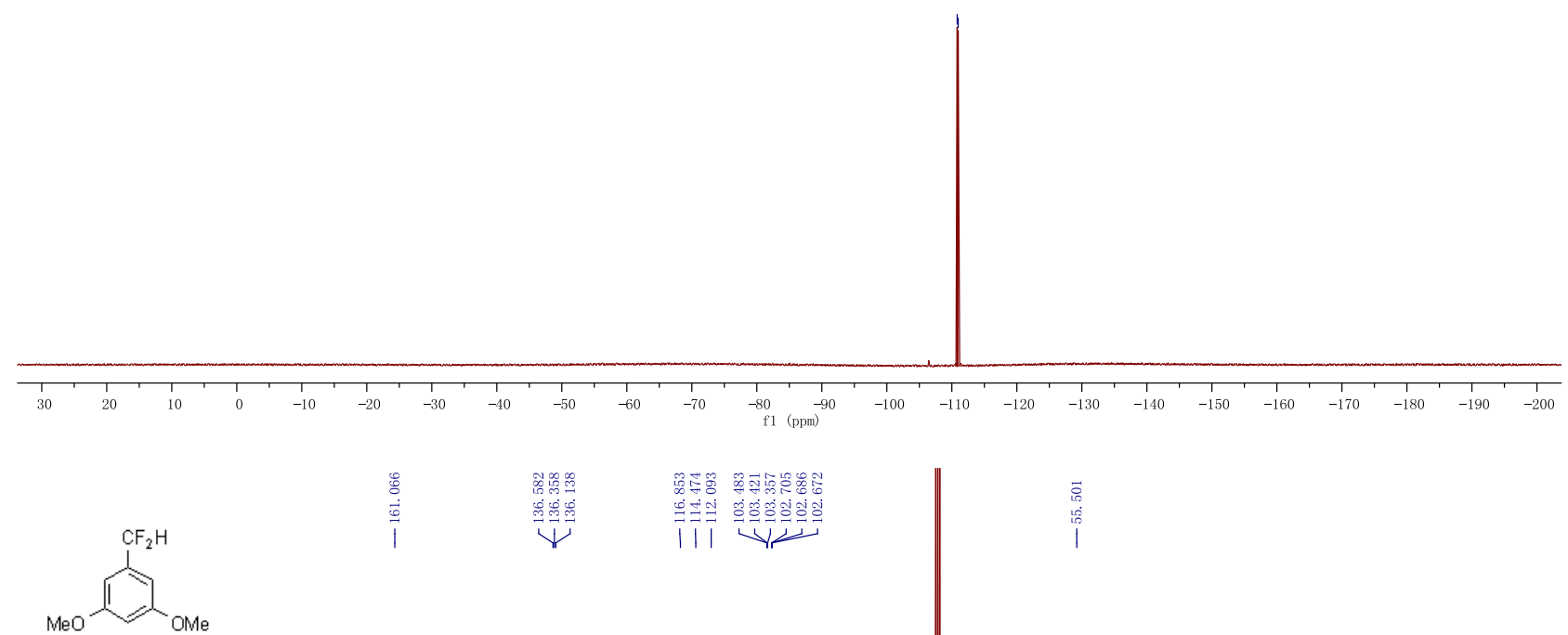

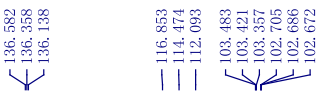

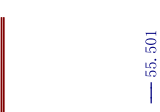

manum 
5-(Difluoromethyl)benzo[d][1,3]dioxole (12).
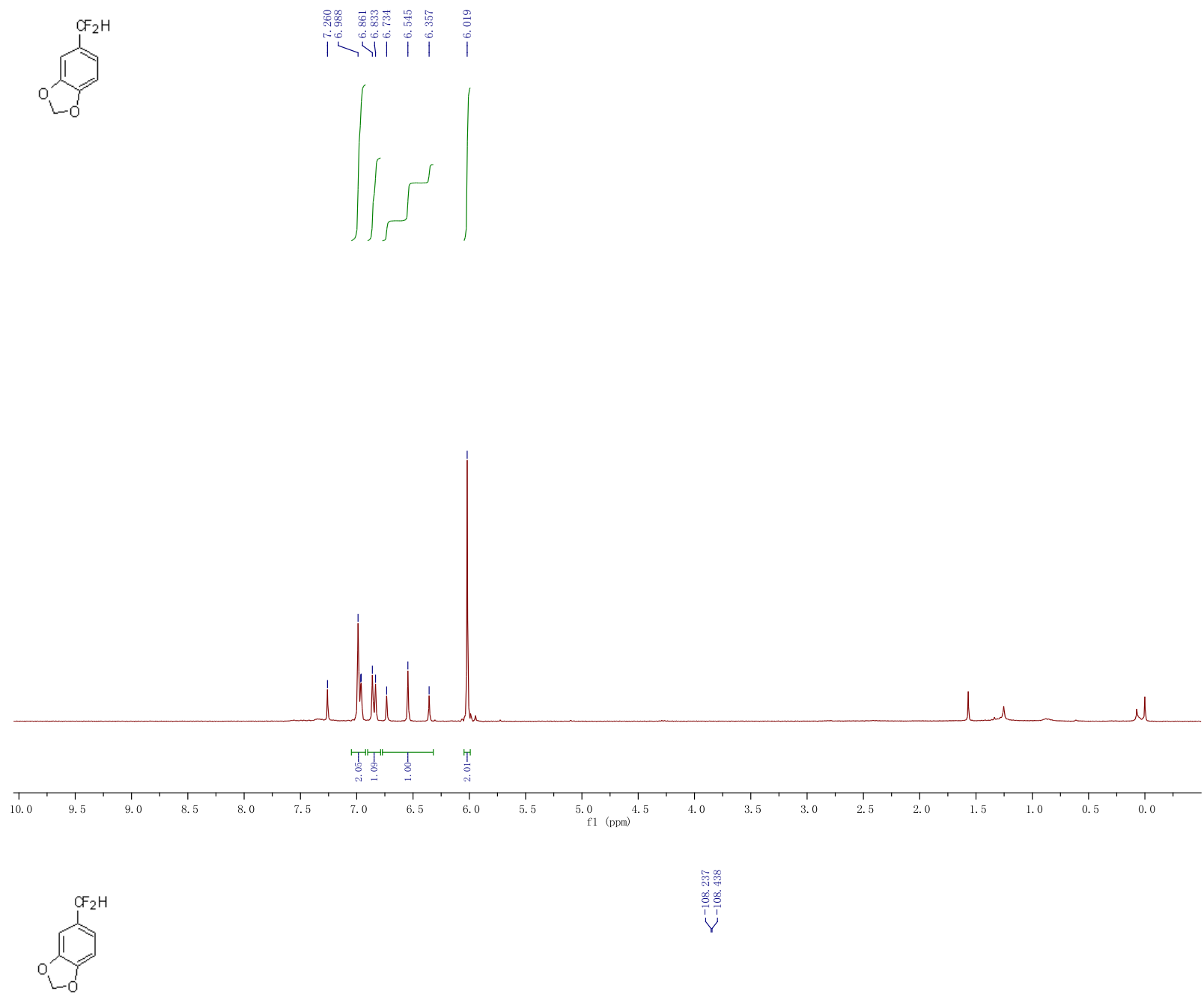

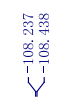

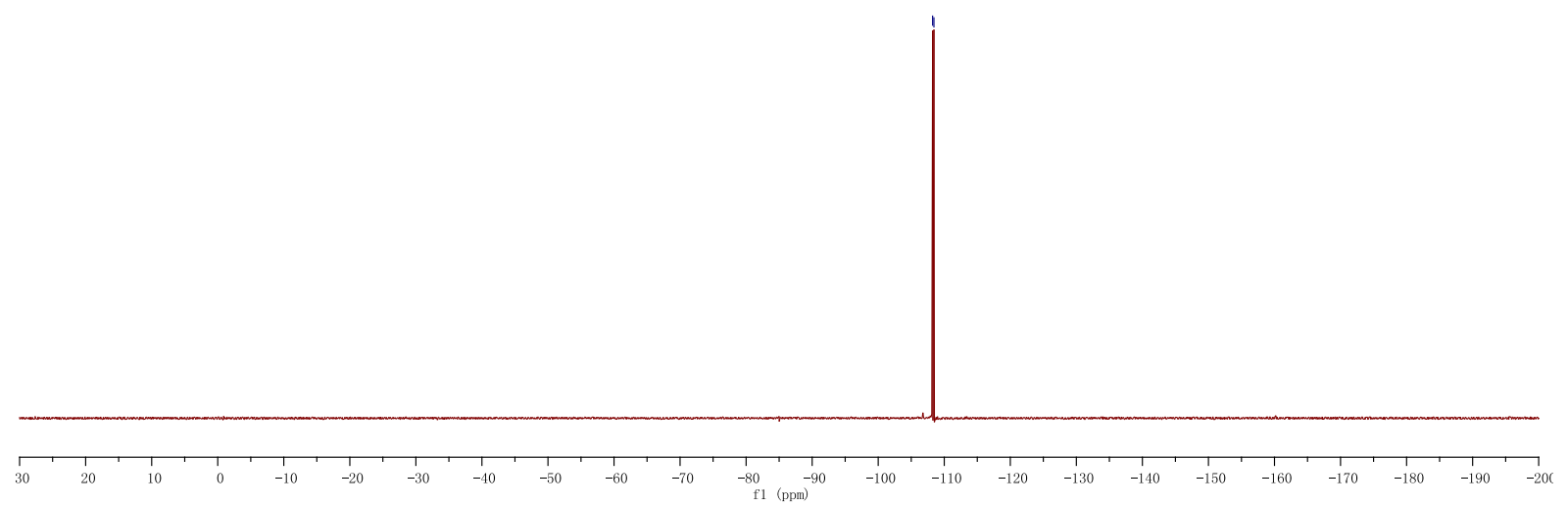




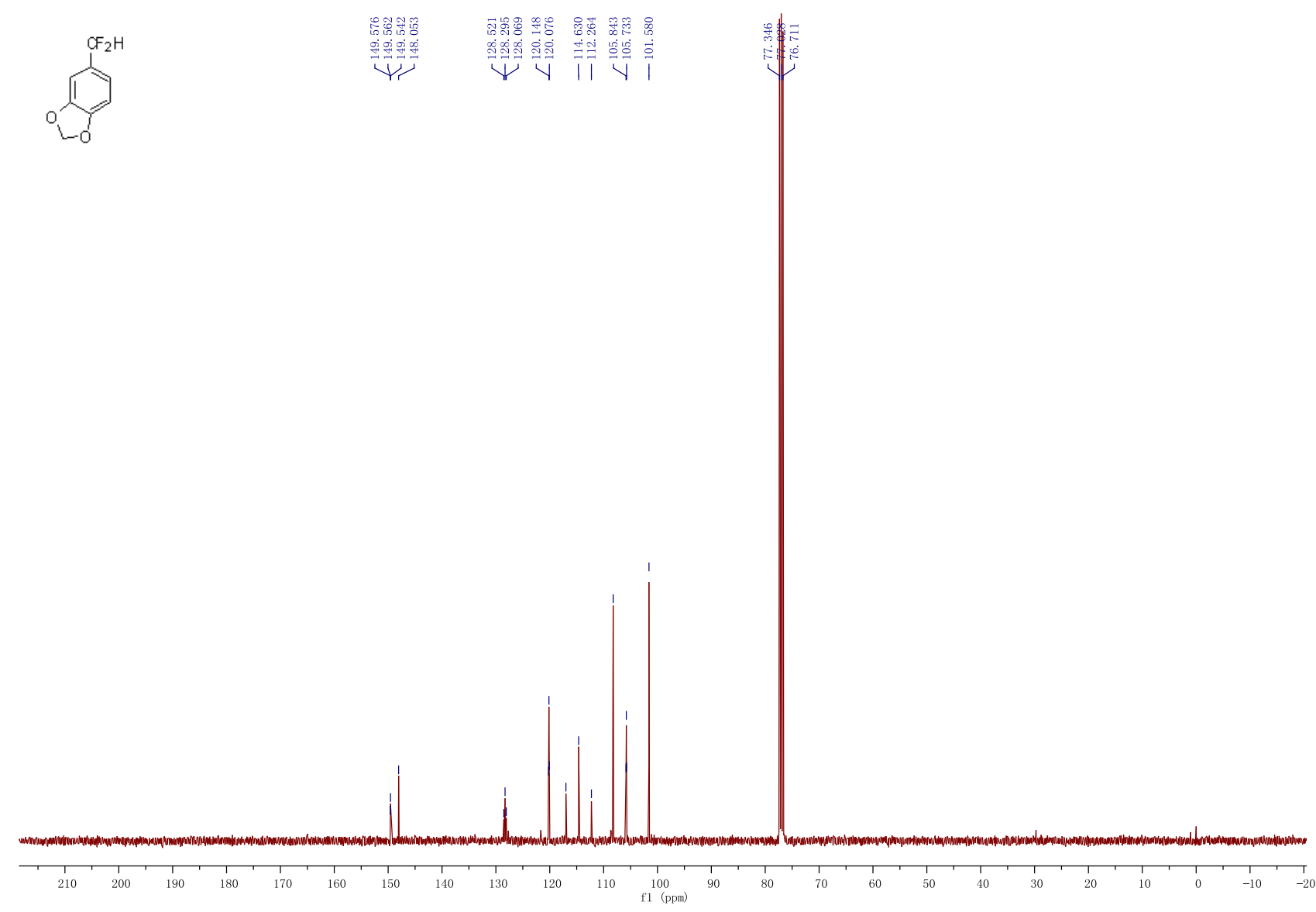

1-((4-(Difluoromethyl)benzyl)oxy)naphthalene (13).
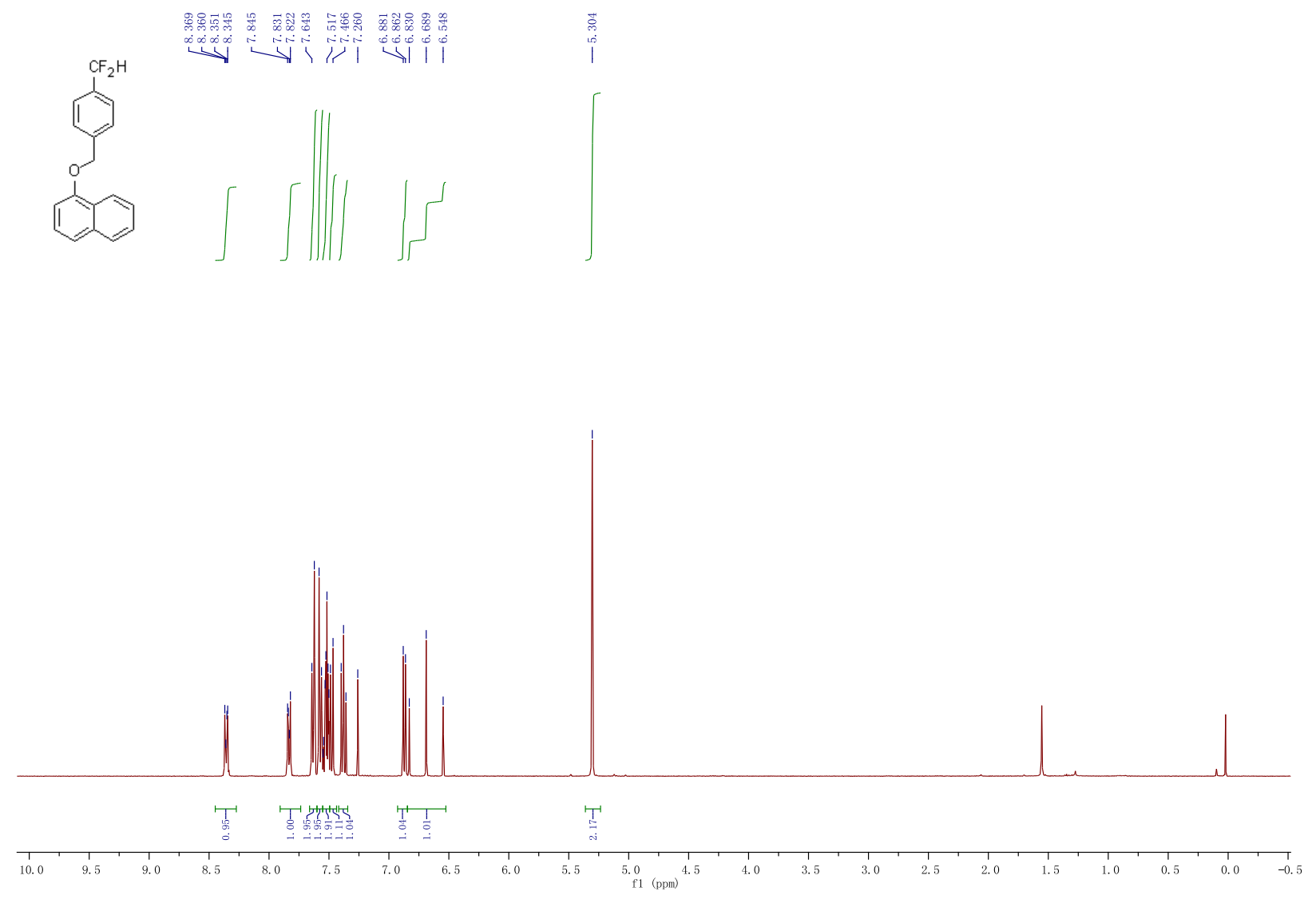

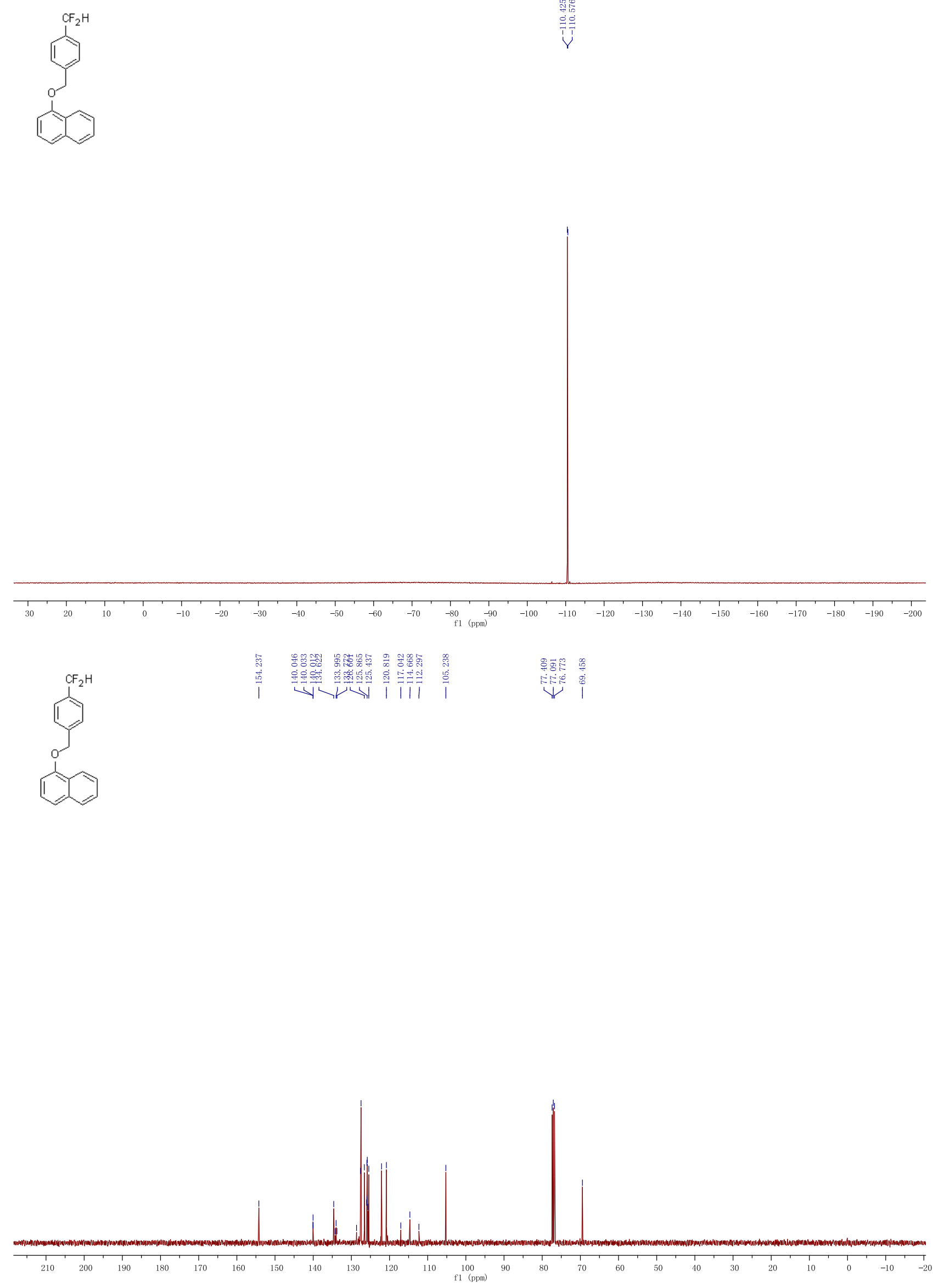
2-(Difluoromethyl)-1,4-dimethylbenzene (14).

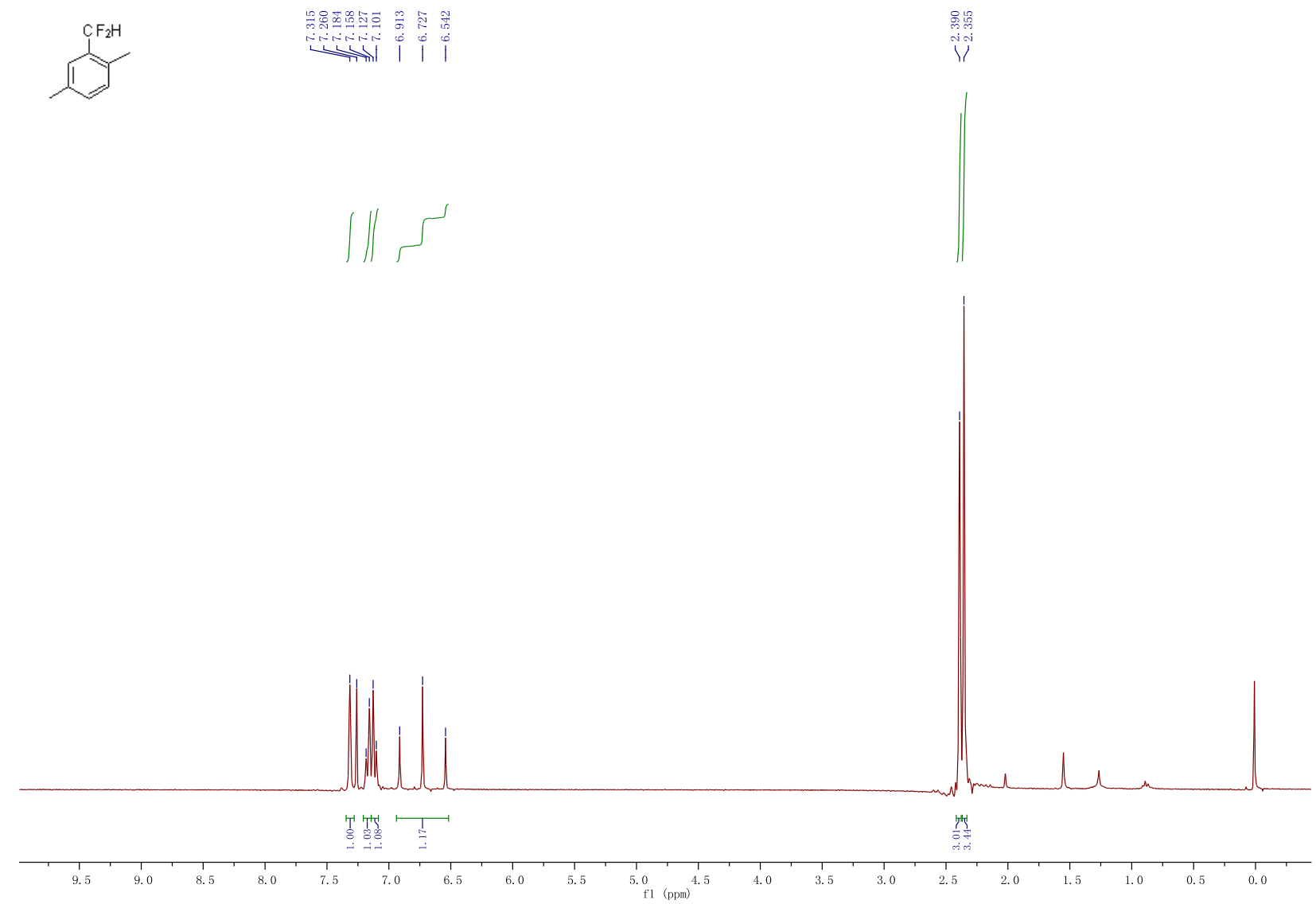

(C)
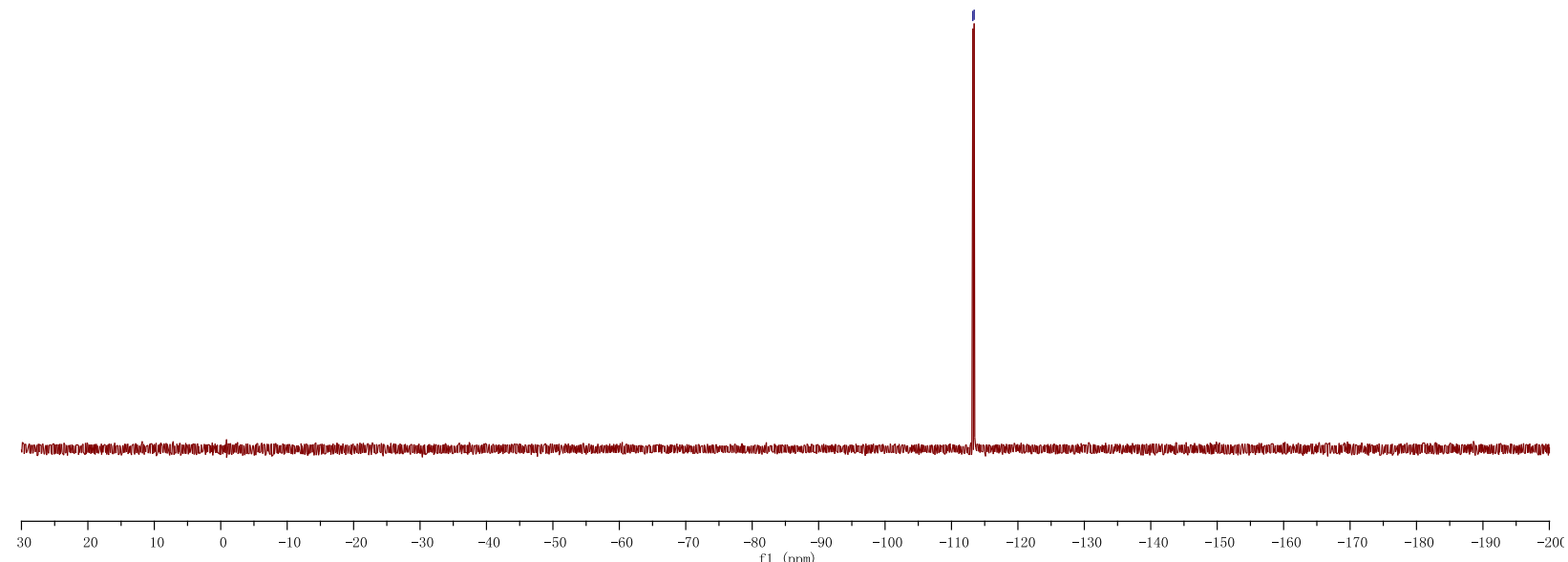
1-(Difluoromethyl)naphthalene (15).
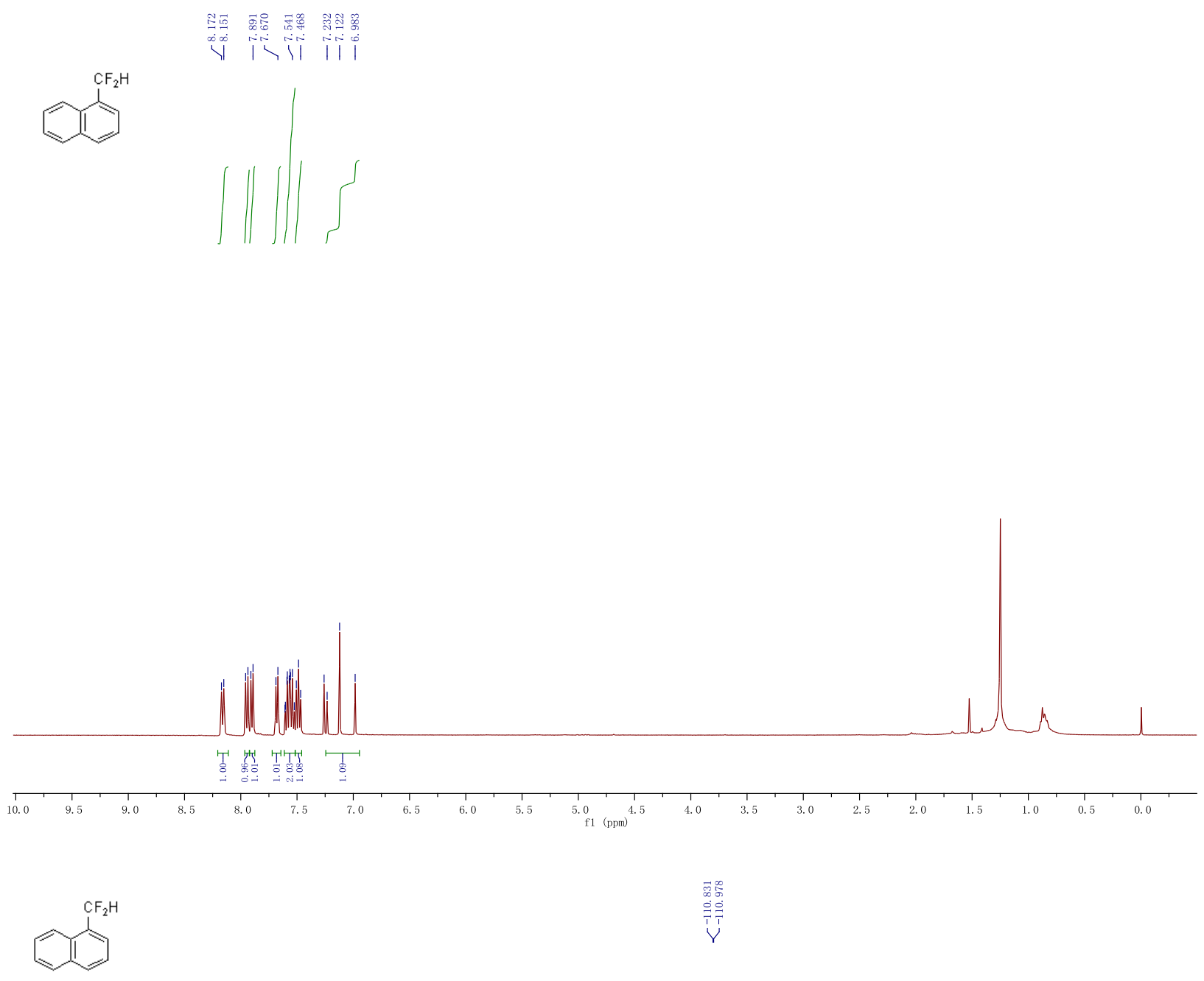

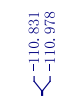

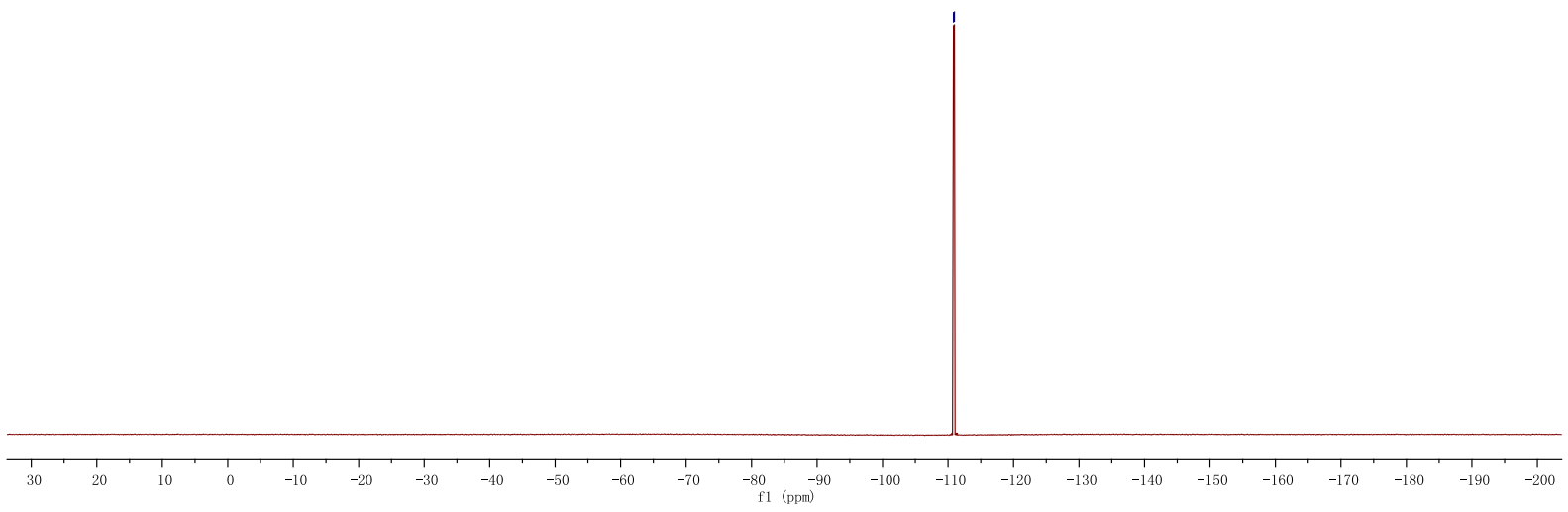

S55 


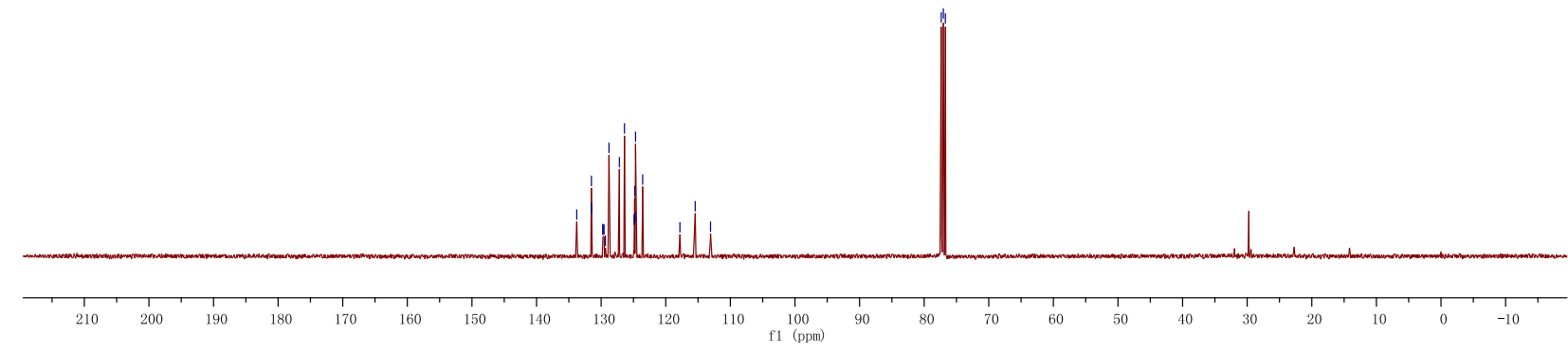

2-(Difluoromethyl)naphthalene (16).
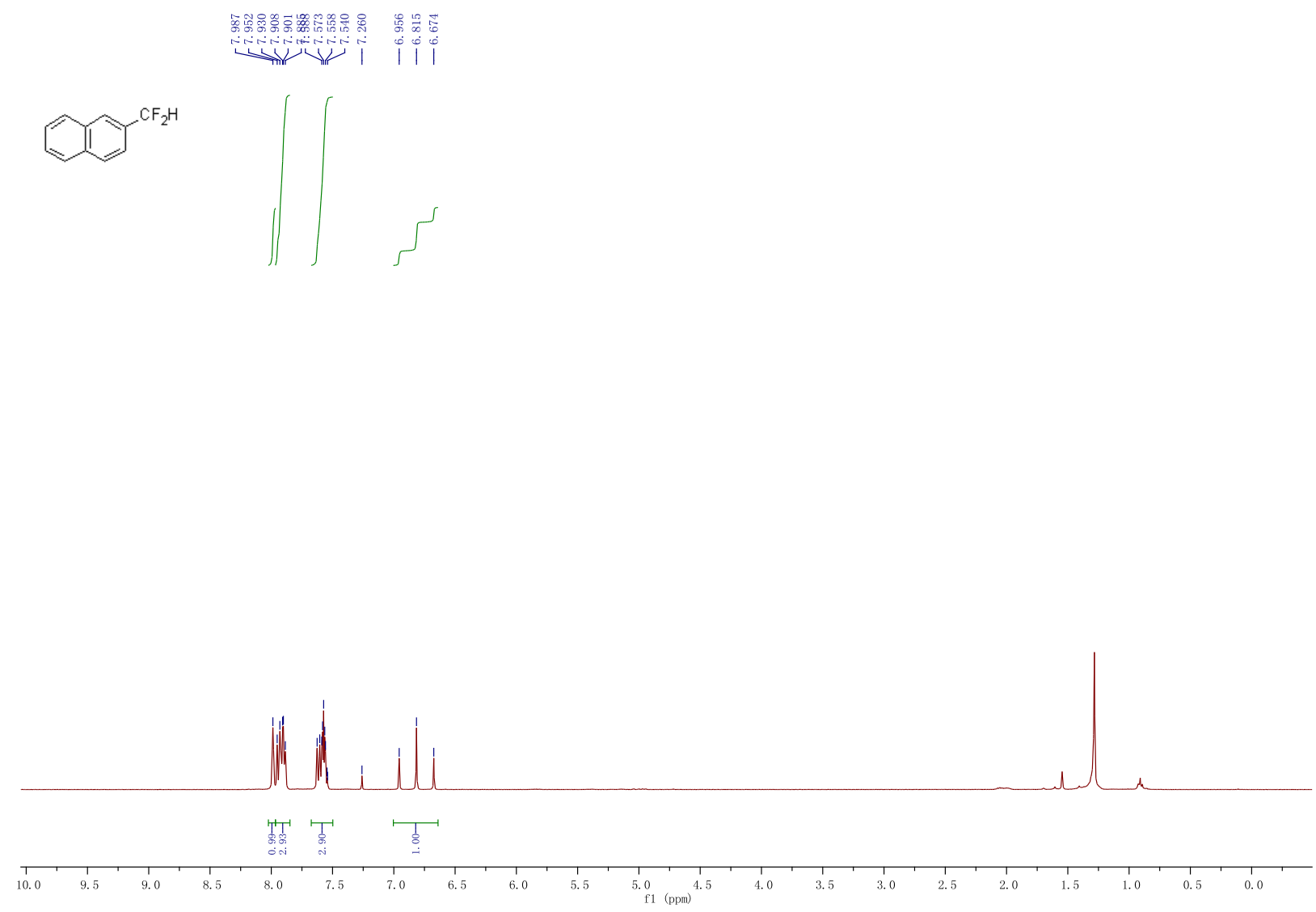
$\overbrace{}^{\mathrm{CF}_{2} \mathrm{H}}$
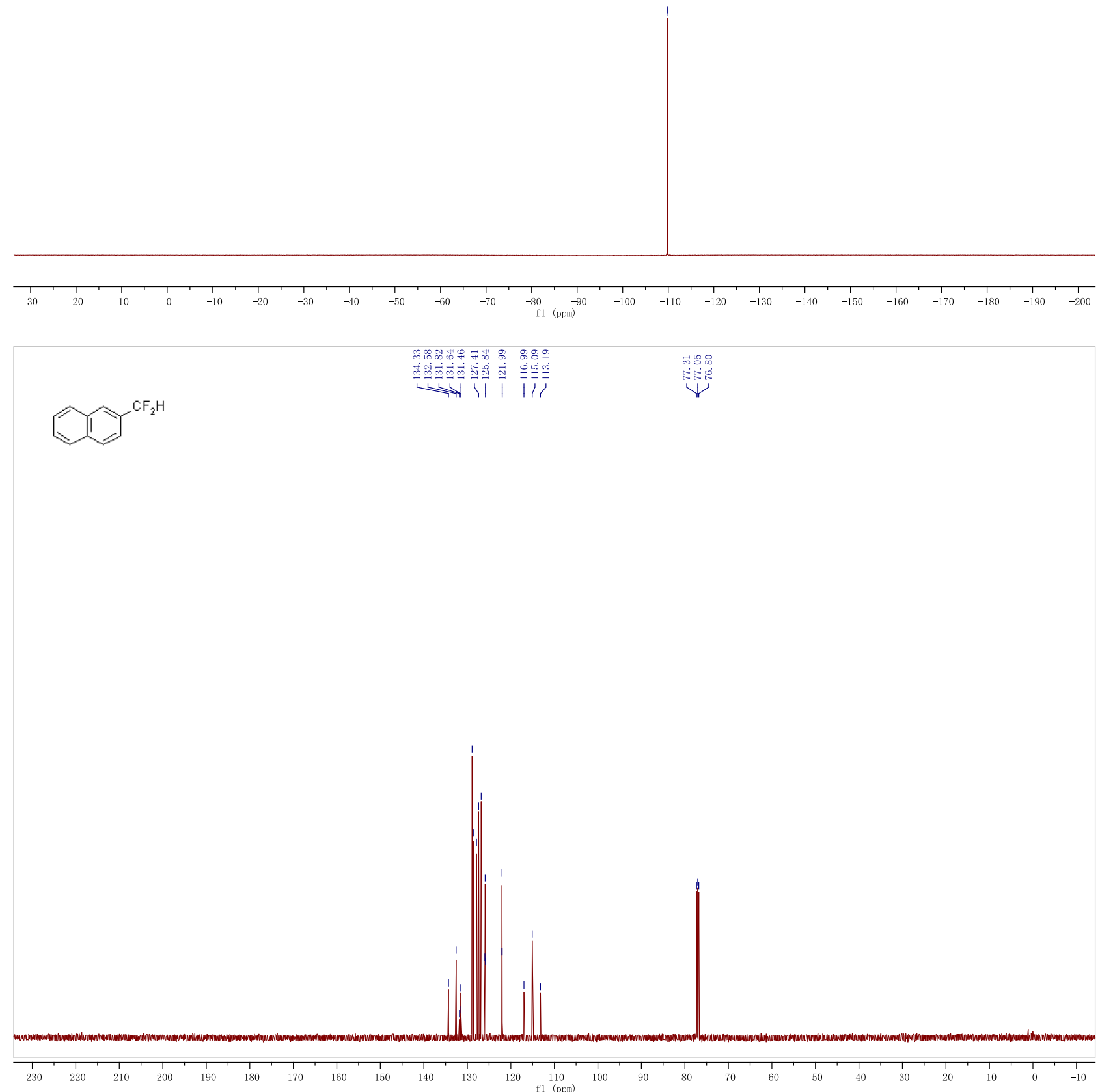
9-(Difluoromethyl)phenanthrene (17).
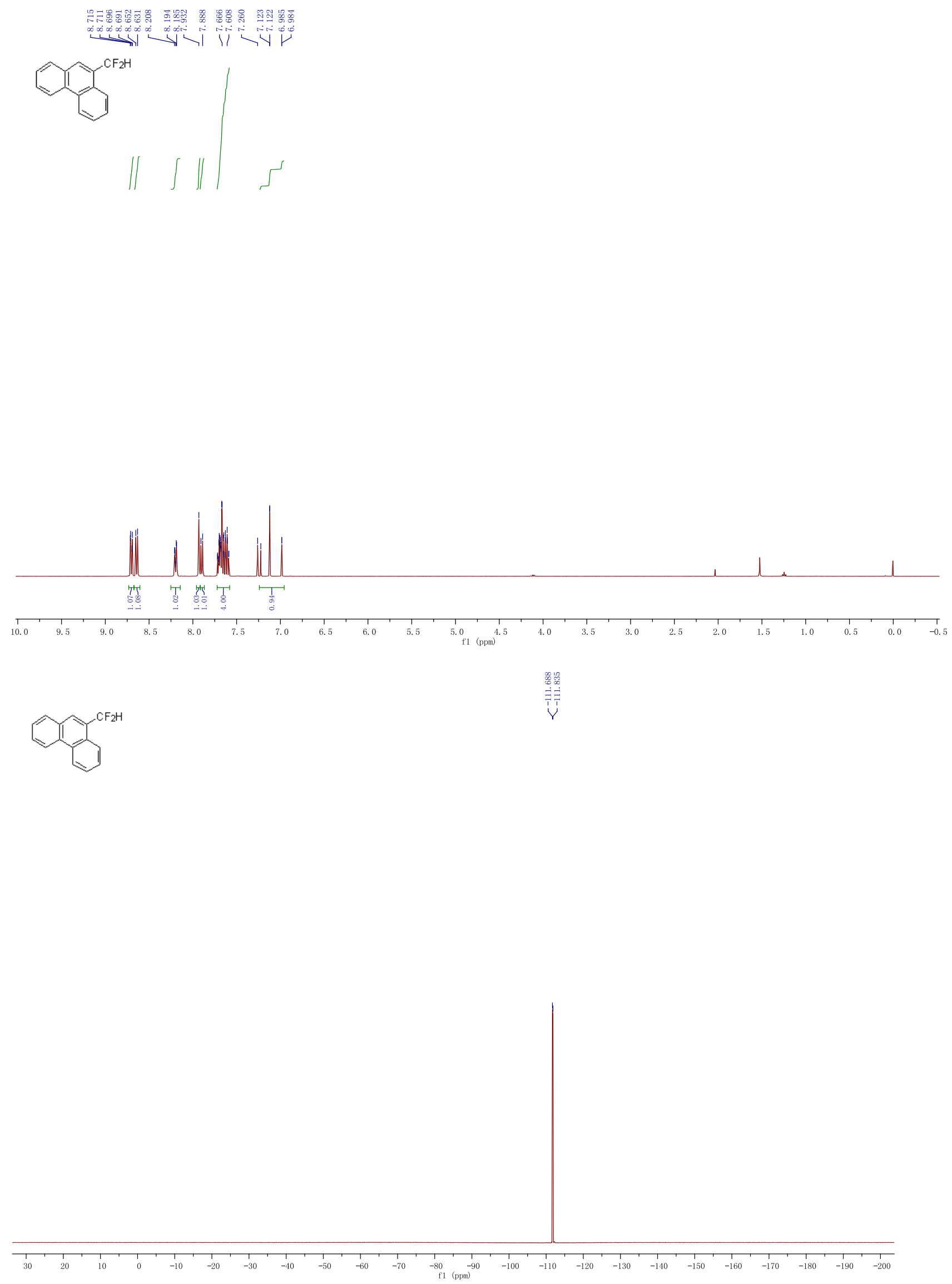
(1)

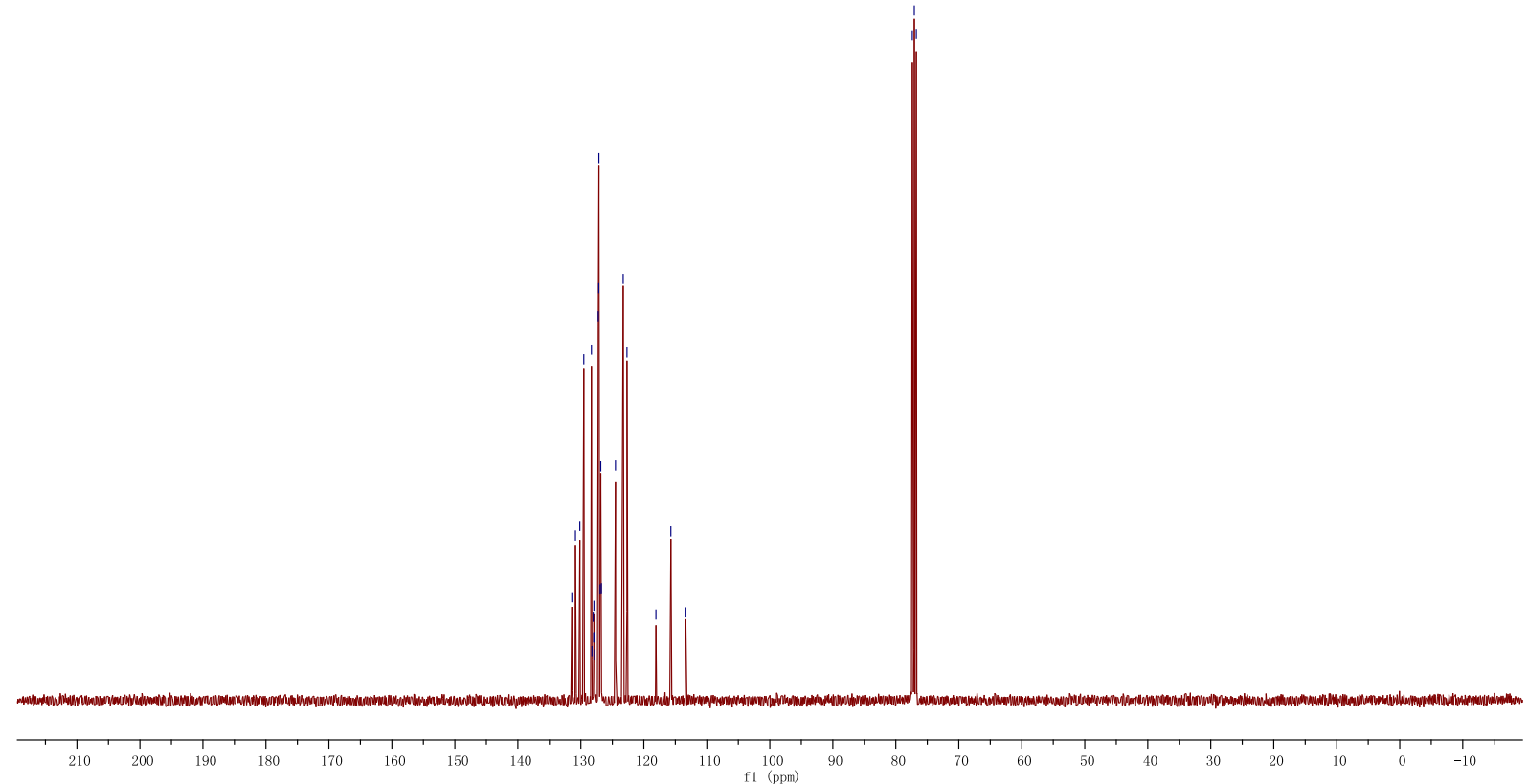

4-(Difluoromethyl)benzaldehyde (18).
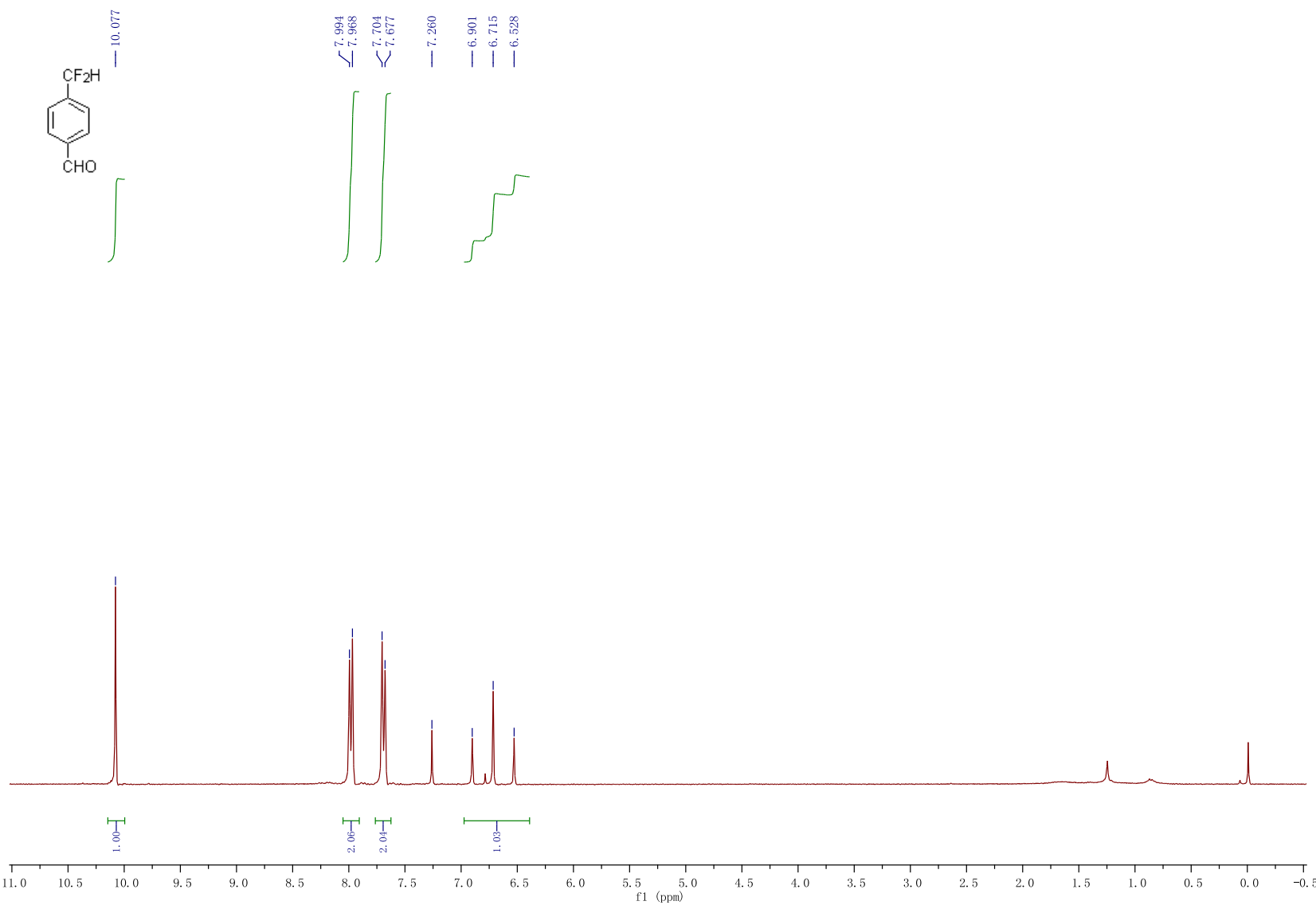

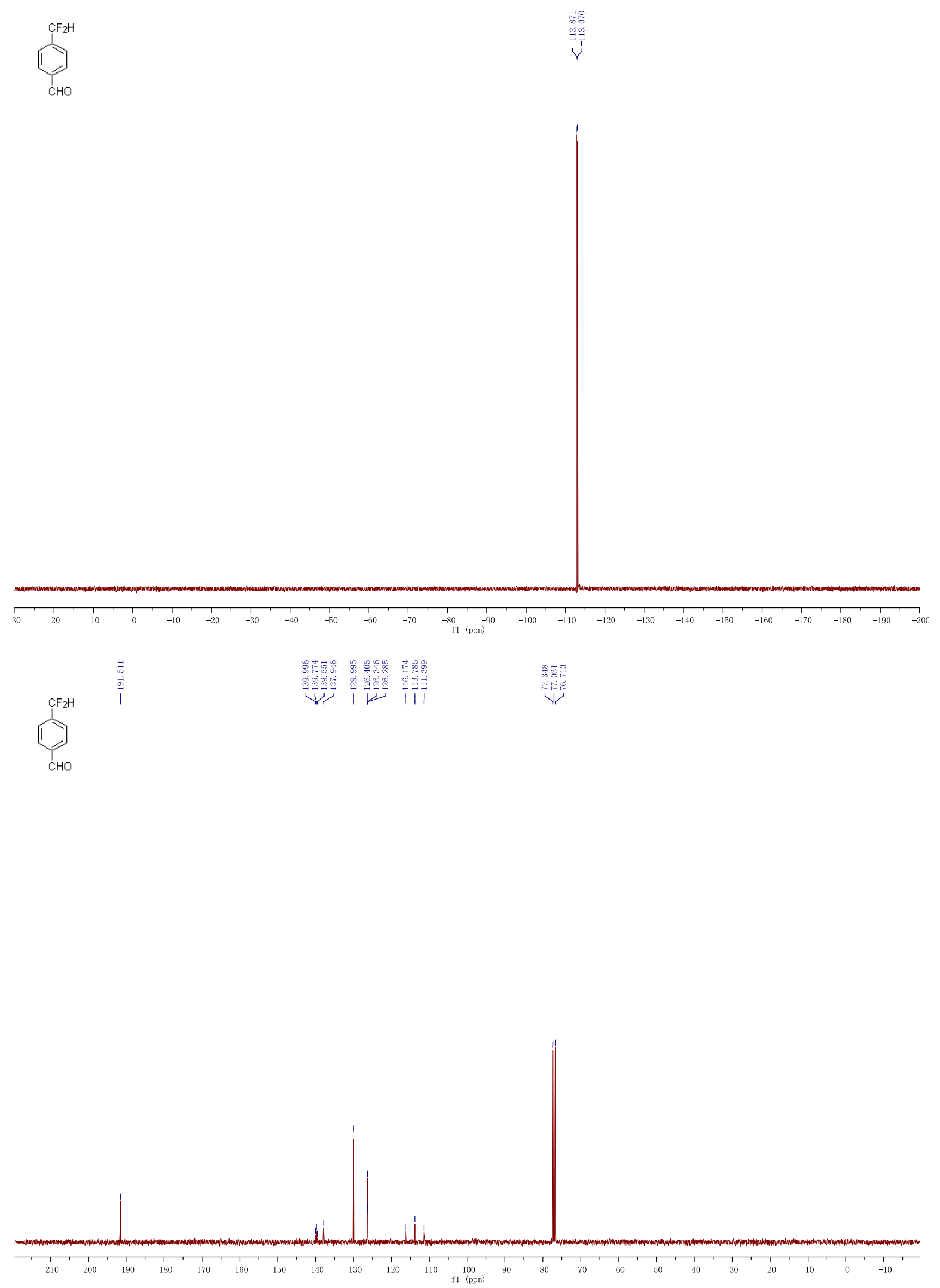
3-(Difluoromethyl)benzaldehyde (19).

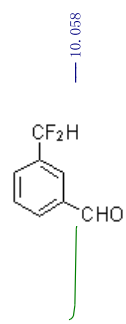

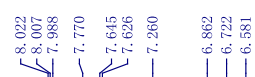
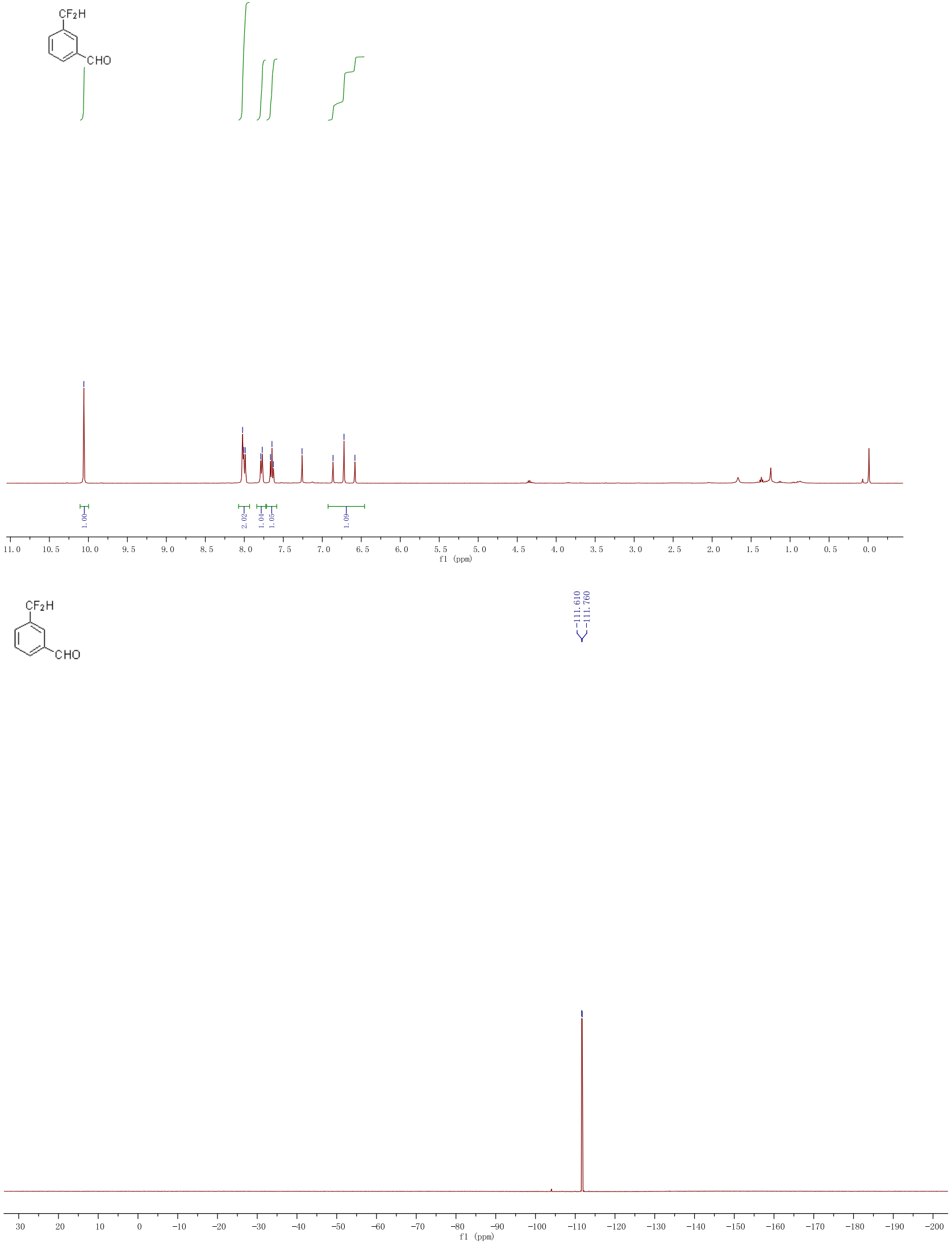
CHO

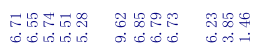

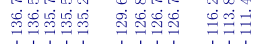

运 ।

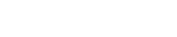

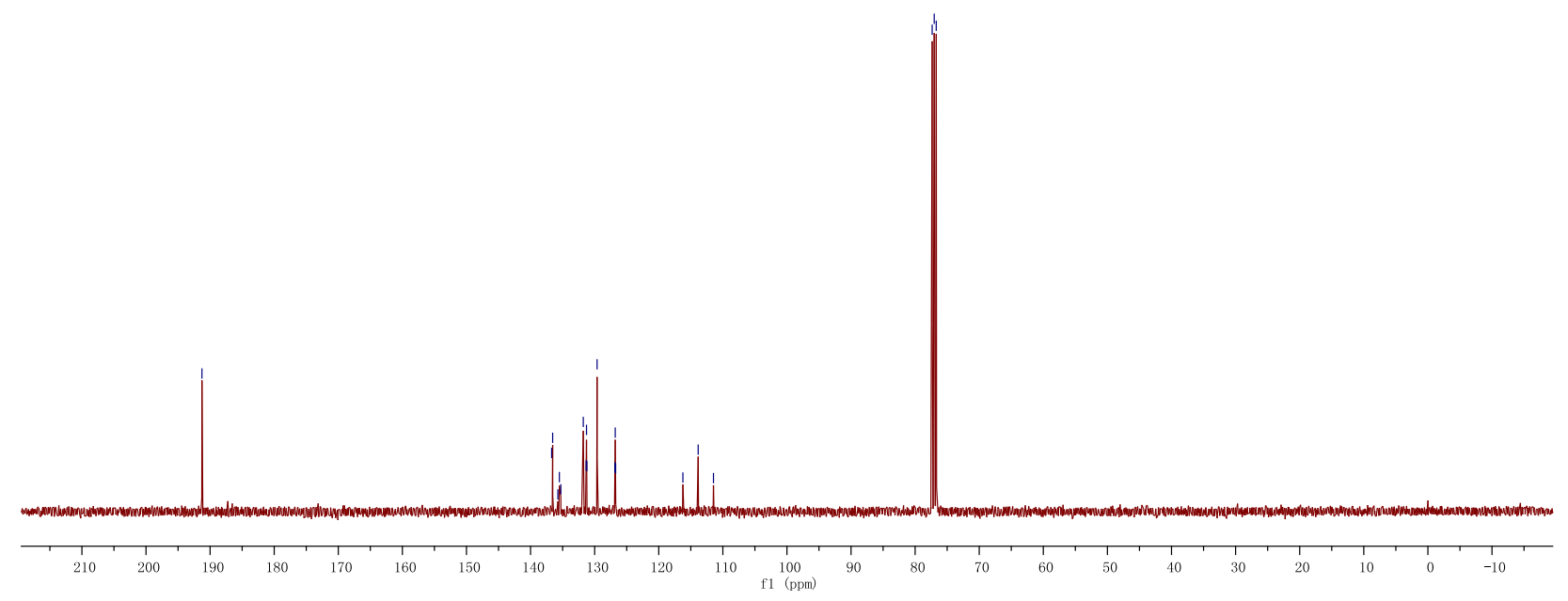

2-(Difluoromethyl)benzaldehyde (20).
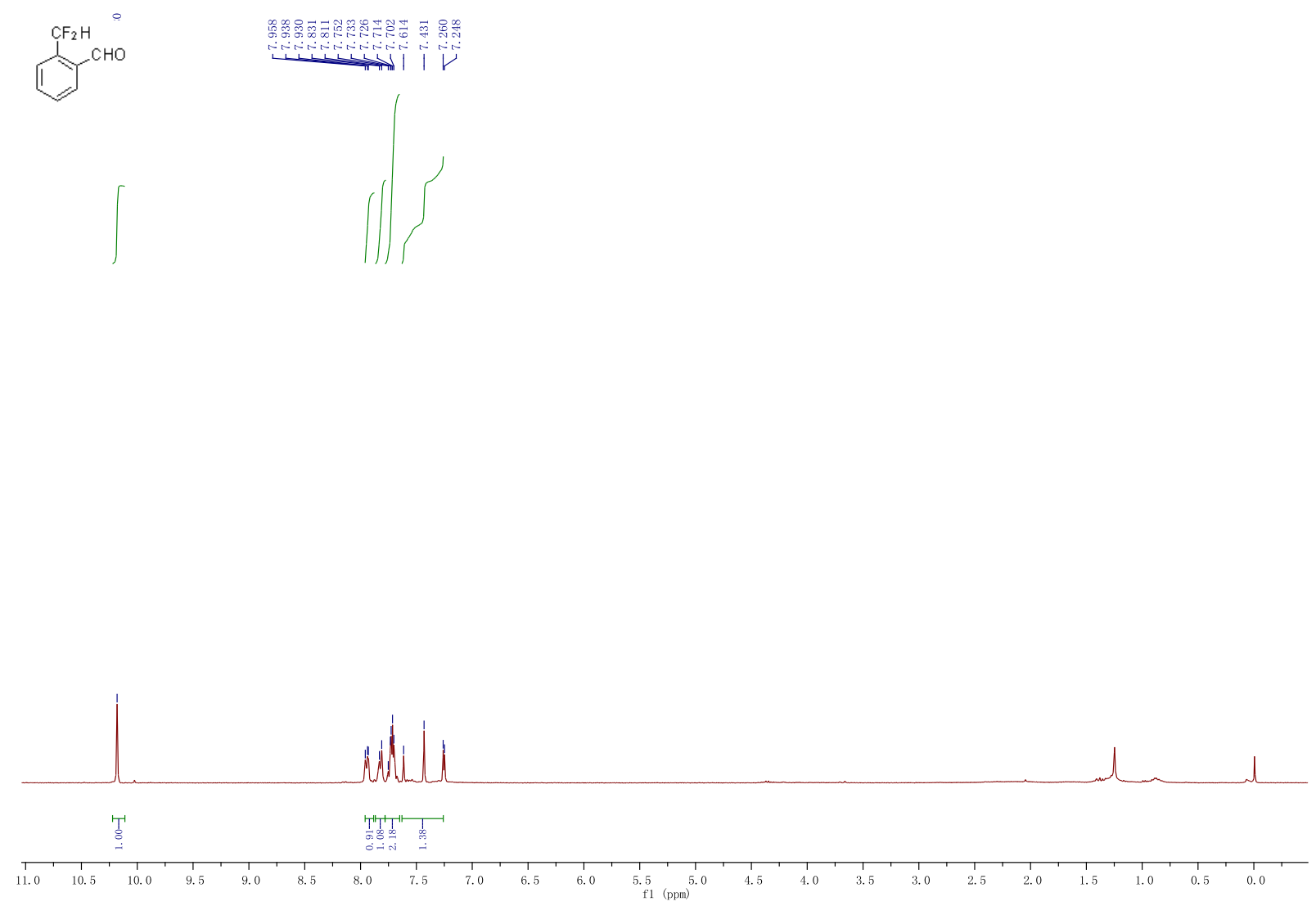
$\mathrm{CF}^{\mathrm{CHO}}$
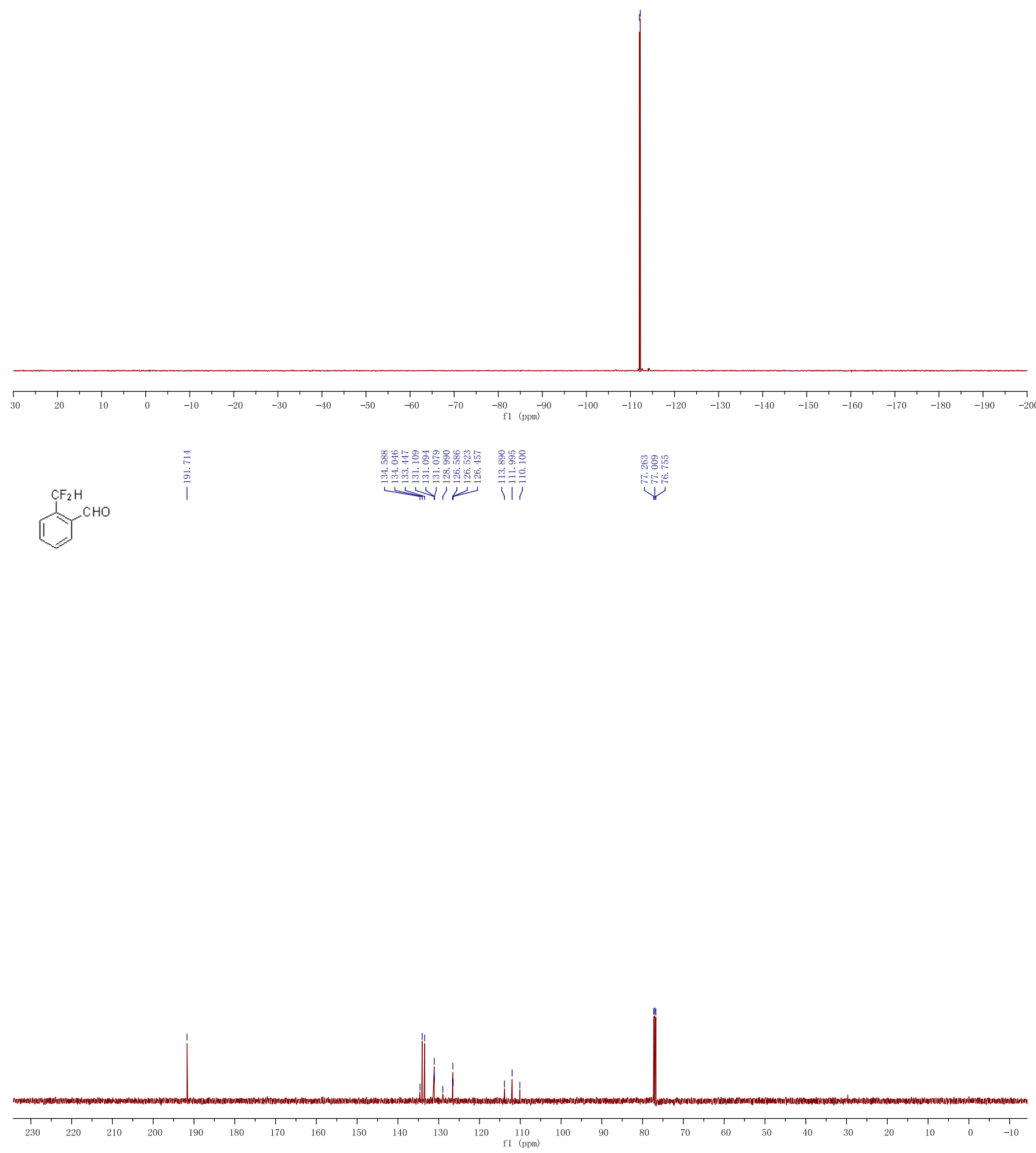
Ethyl 4-(difluoromethyl)benzoate (21).
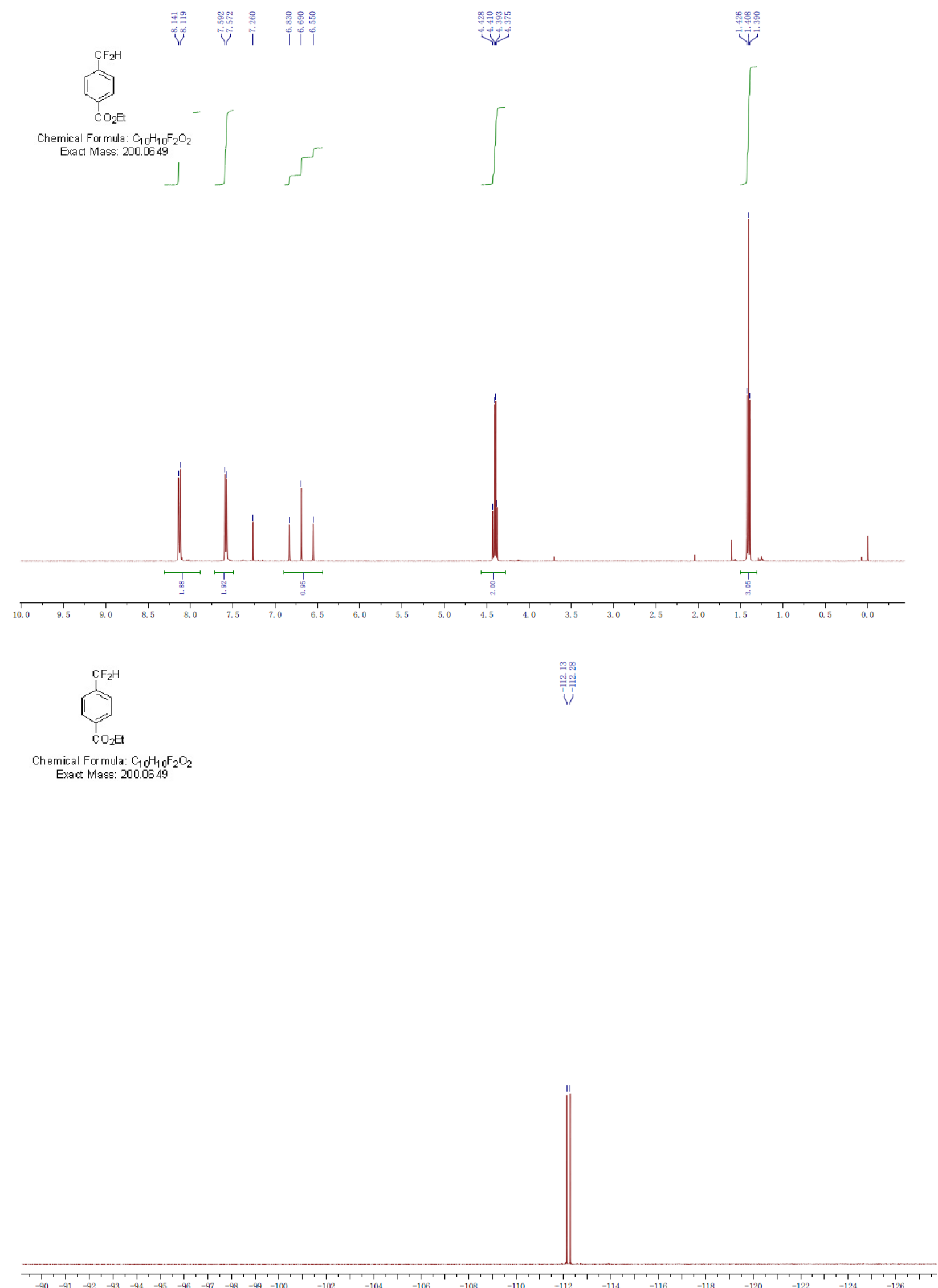
CO

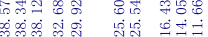

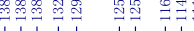

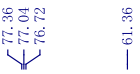

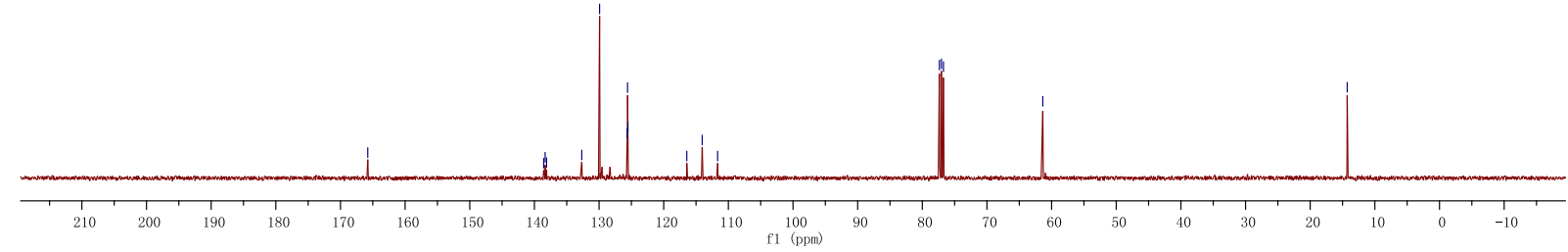

Ethyl 3-(difluoromethyl)benzoate (22).

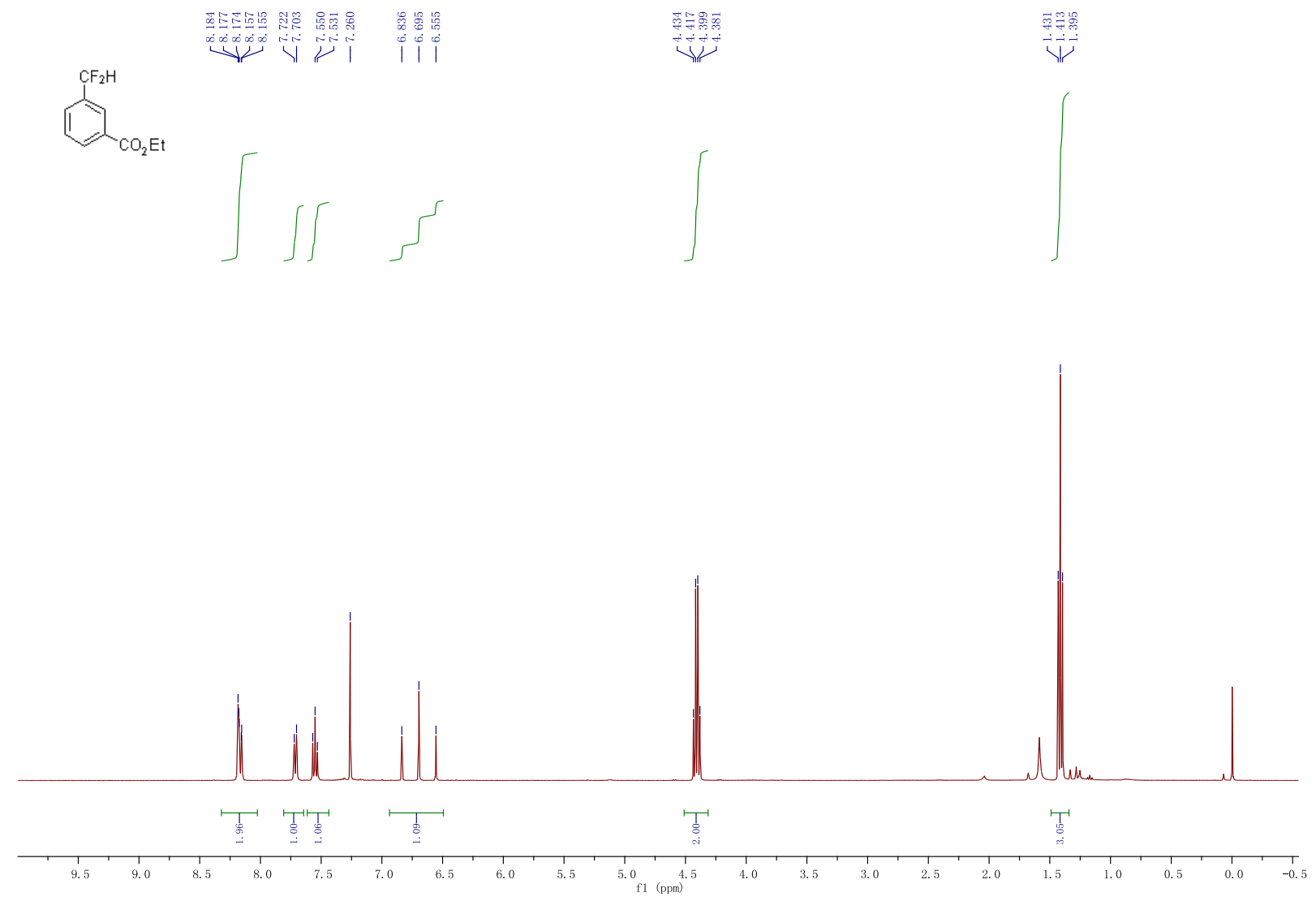


$\mathrm{CO}_{2} \mathrm{Et}$

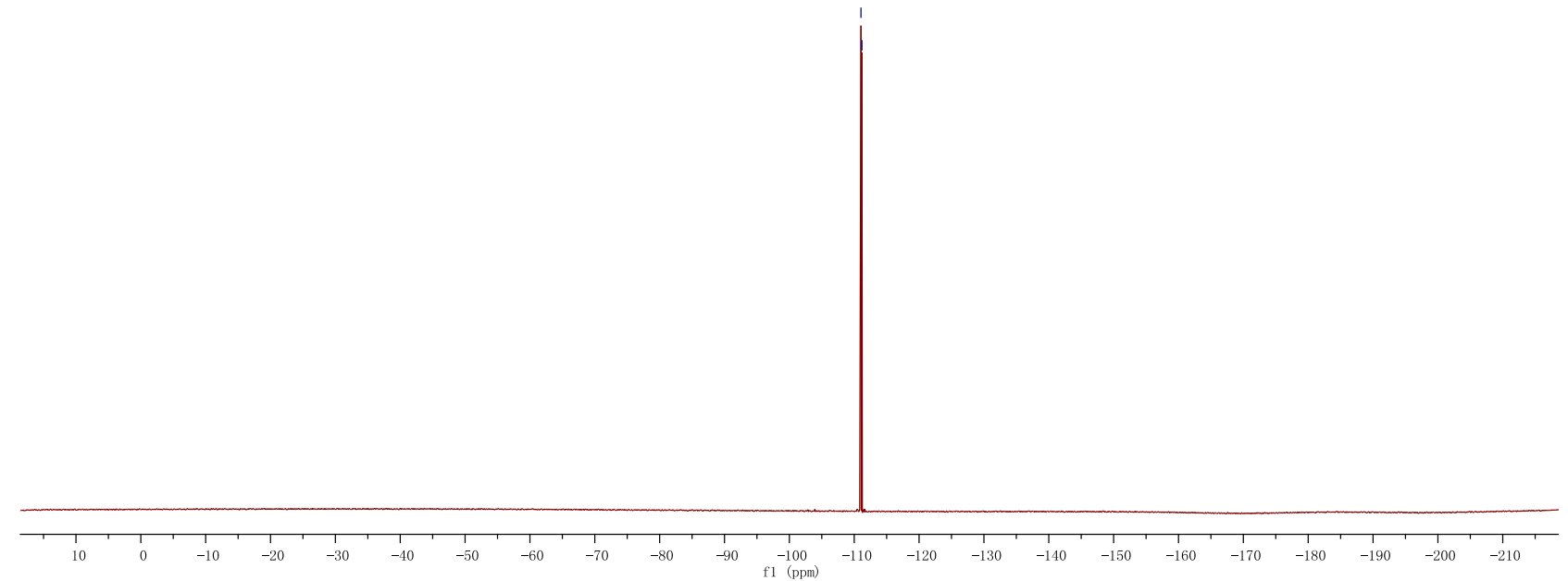

$\mathrm{CO}_{2} \mathrm{Et}$

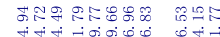

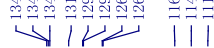

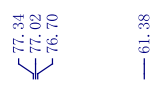

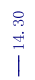

i

-

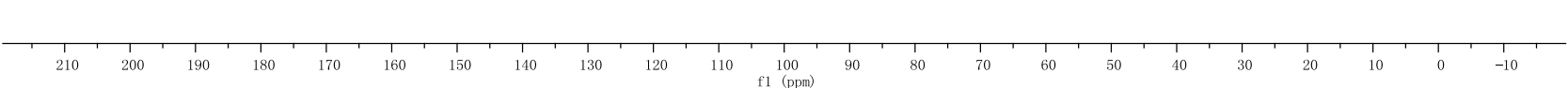


1-(4-(Difluoromethyl)phenyl)ethanone (23).
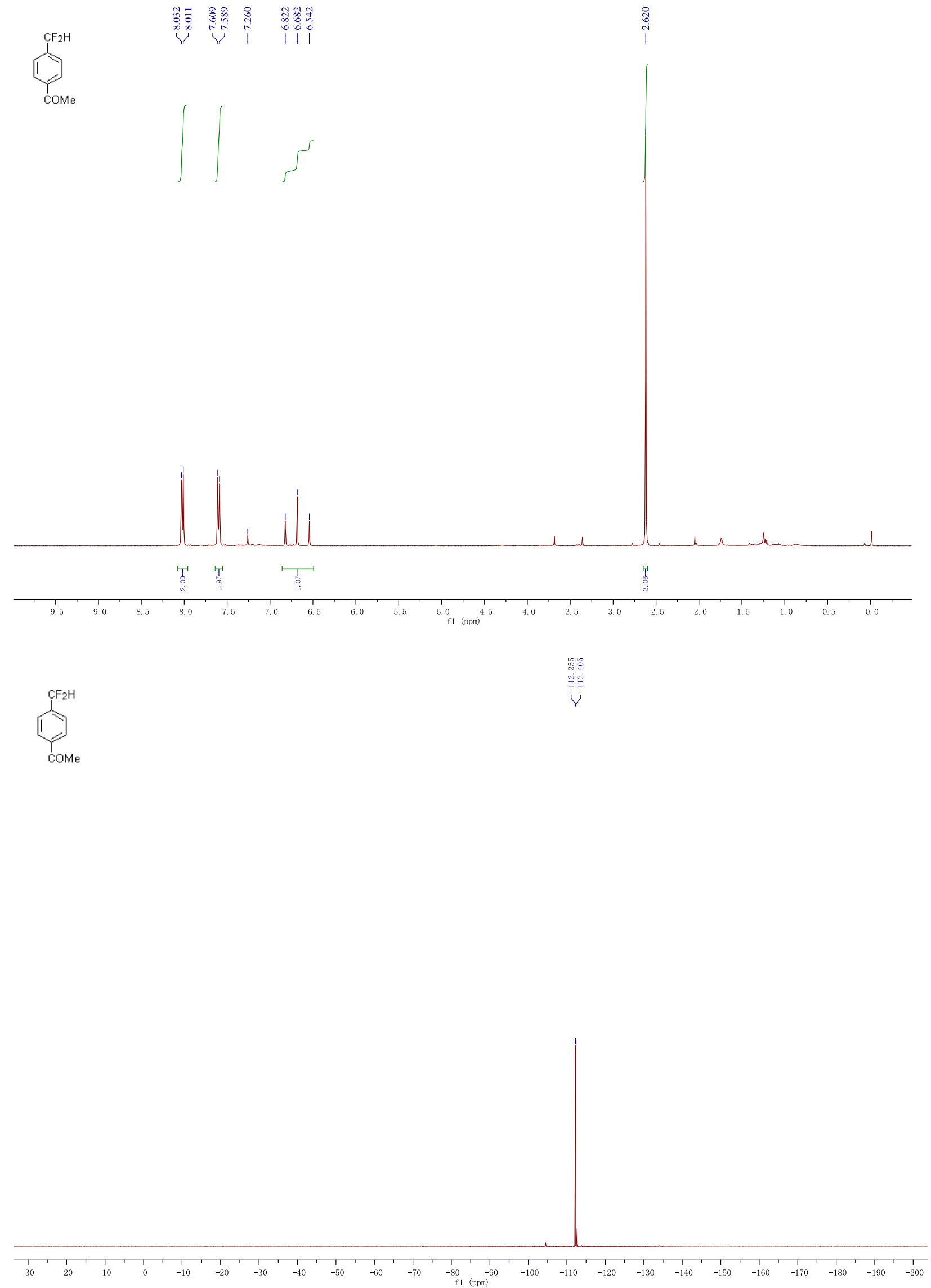

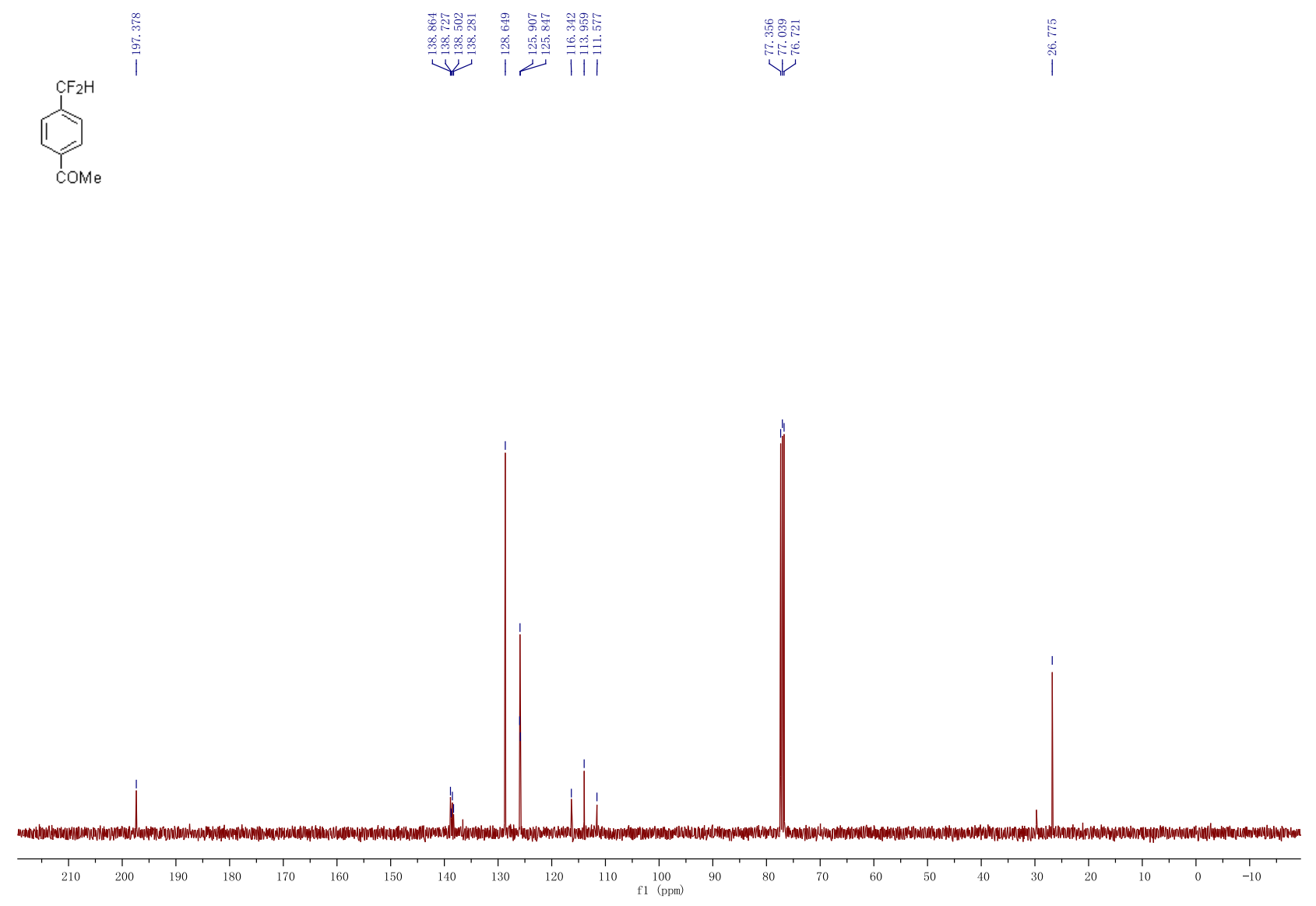

1-(3-(Difluoromethyl)phenyl)ethanone (24).

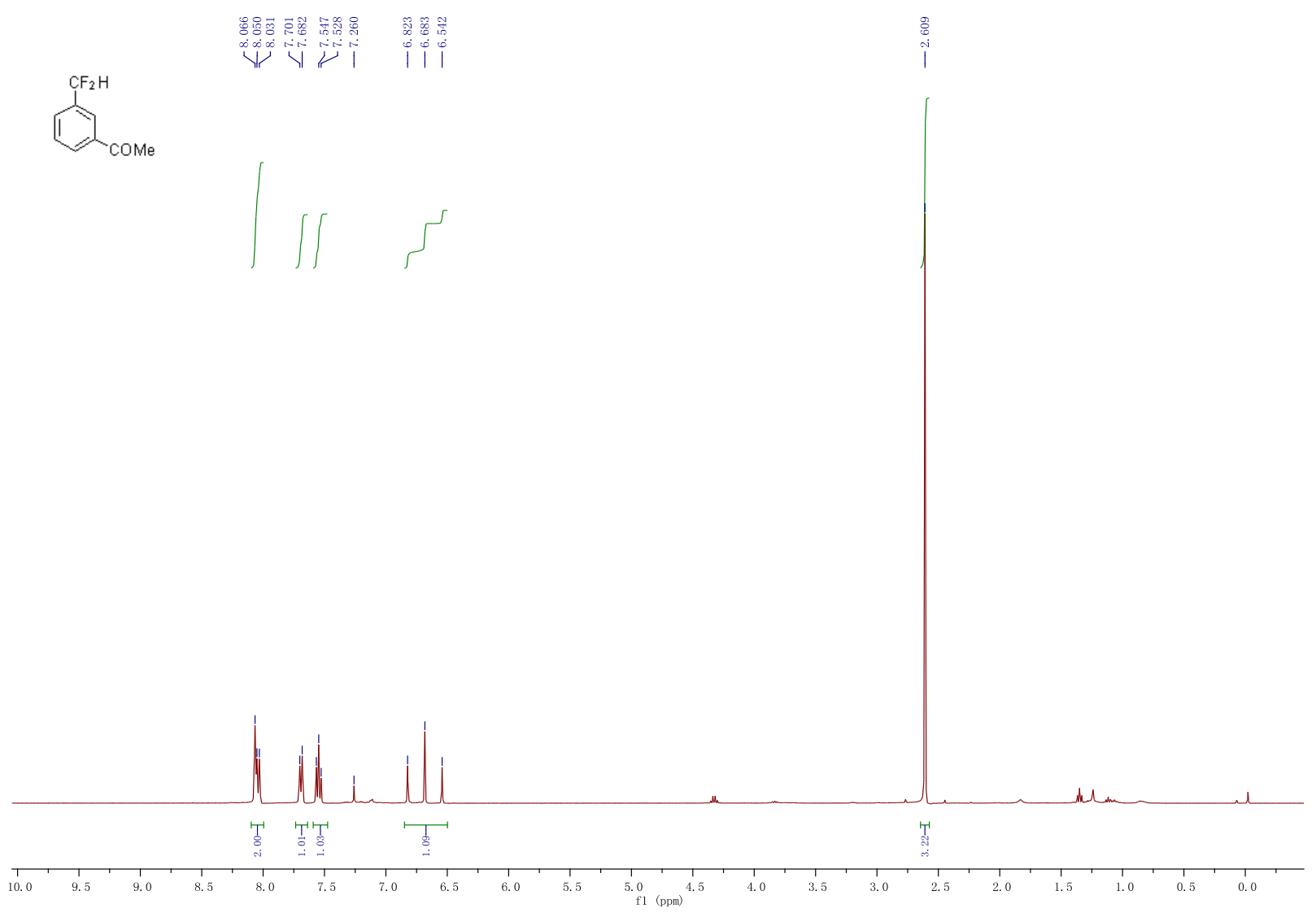


$\mathrm{COMe}_{\mathrm{Me}}^{\mathrm{CF}_{2} \mathrm{H}}$
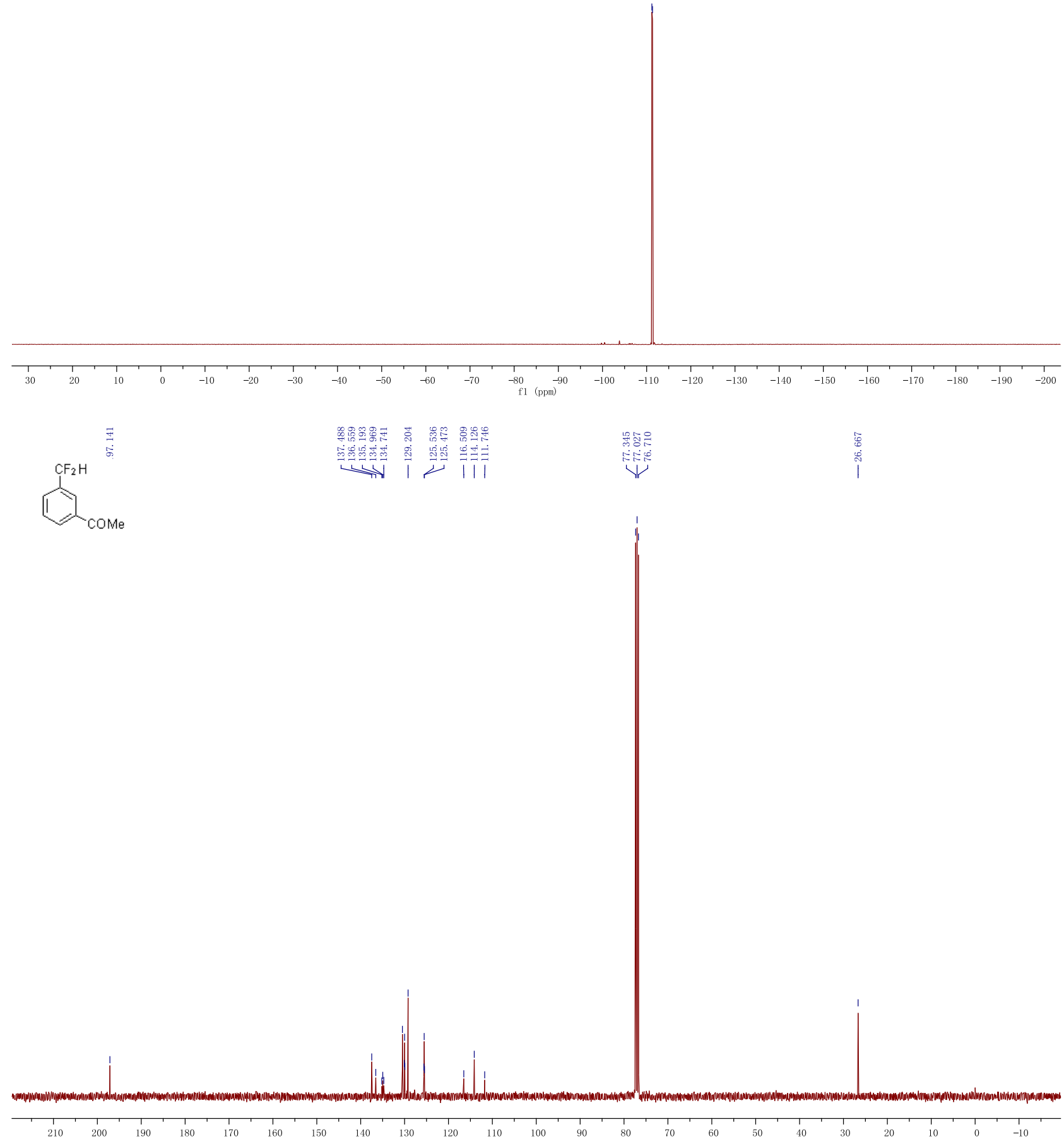
3-(Difluoromethyl)benzonitrile (25).

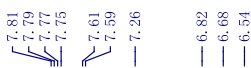

$\mathrm{CHN}_{\mathrm{CN}}^{\mathrm{CF}_{2} \mathrm{H}}$
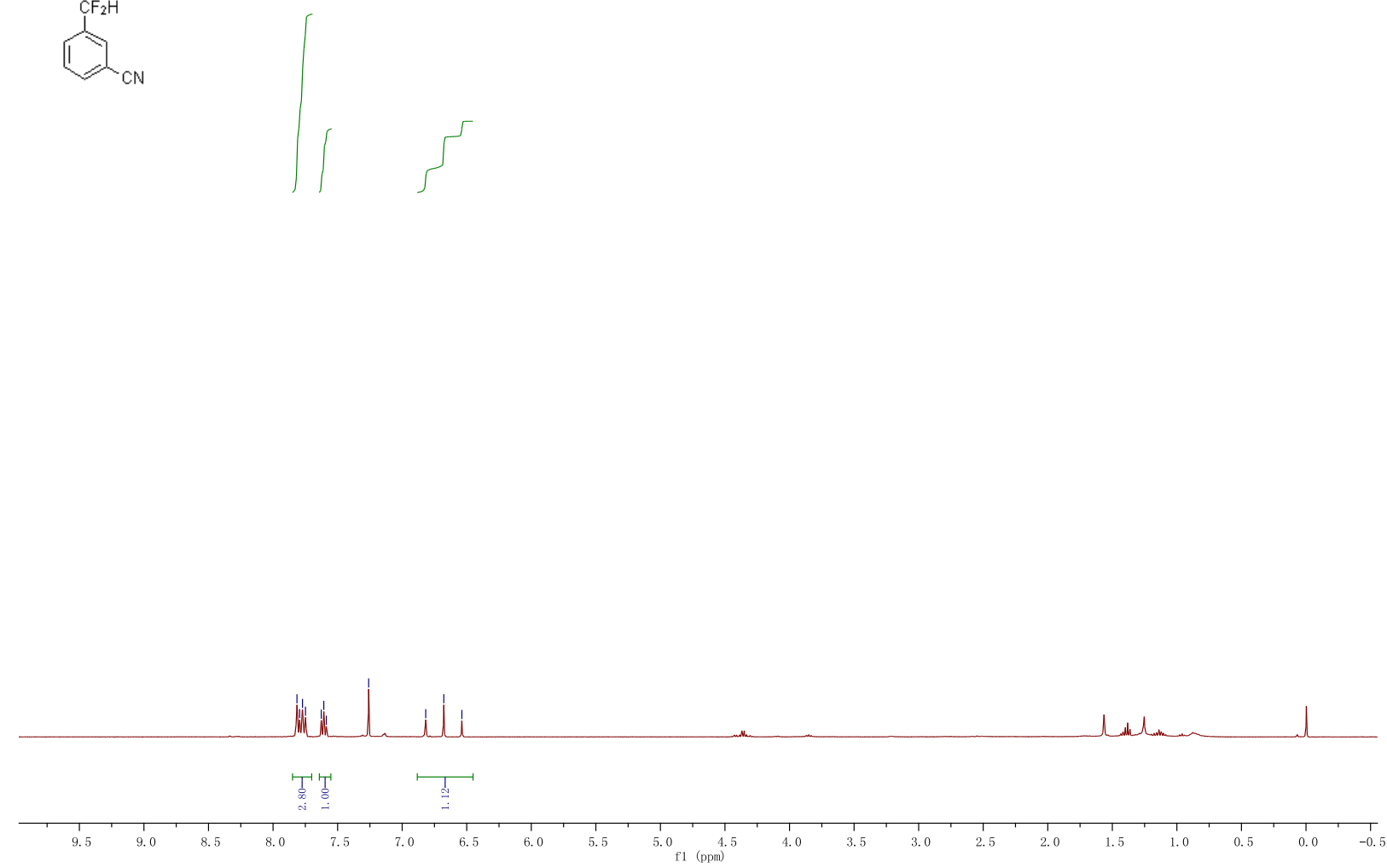

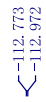

CN

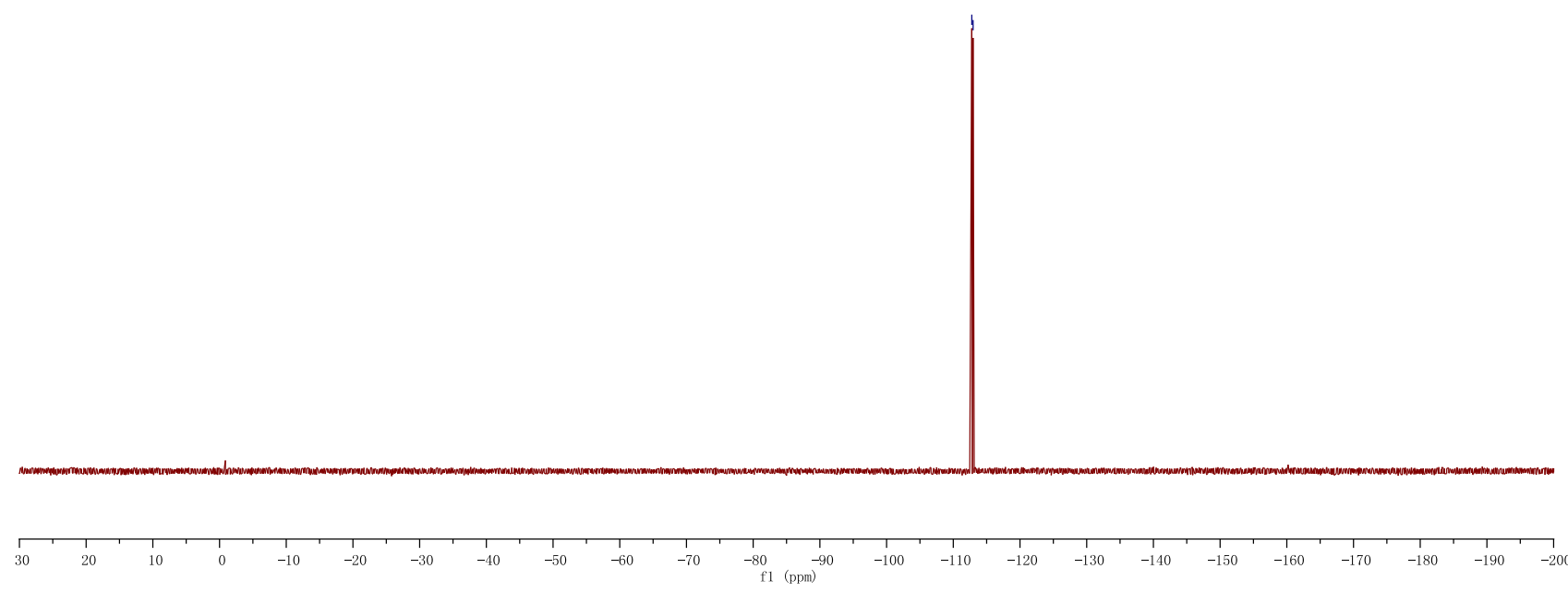


1-(Difluoromethyl)-4-(trifluoromethyl)benzene (26). (Due to the low boil point of 26 , a crude ${ }^{19} \mathrm{~F}$ NMR was provided)
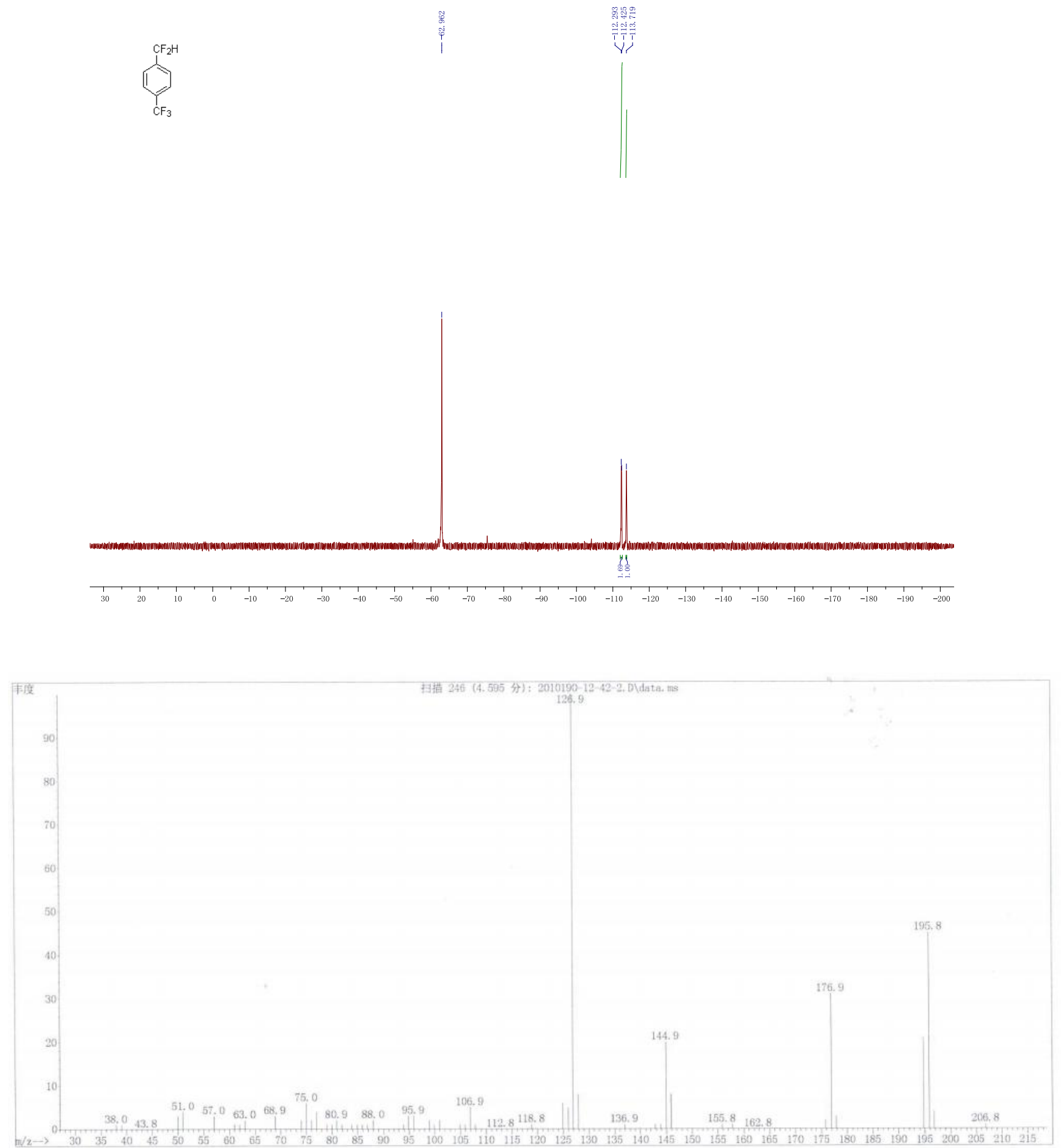
1-(Difluoromethyl)-3-(trifluoromethyl)benzene (27) (Due to the low boil point of 27, a crude ${ }^{19} \mathrm{~F}$ NMR was provided)
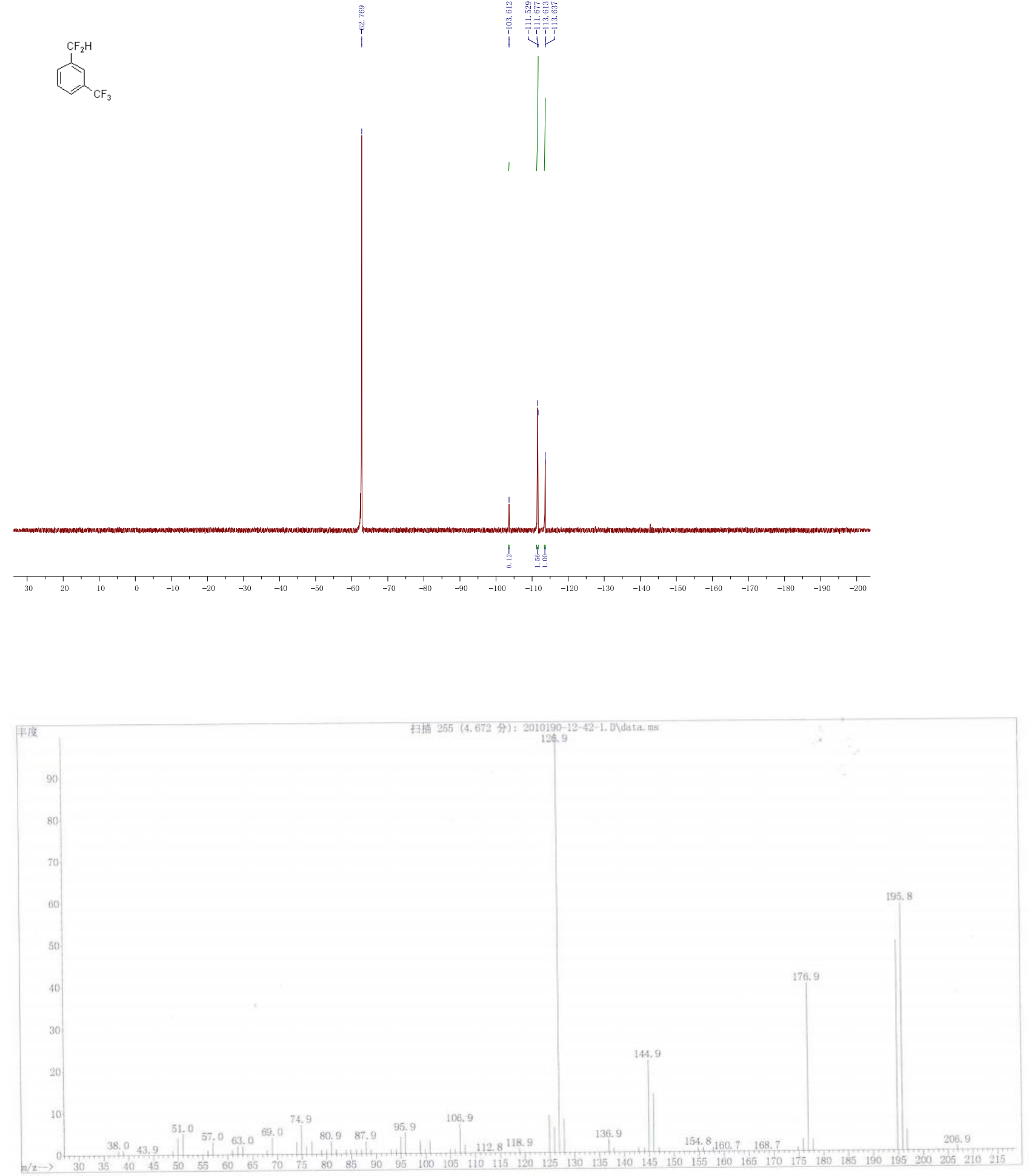
1,3-dichloro-5-(difluoromethyl)benzene (28).
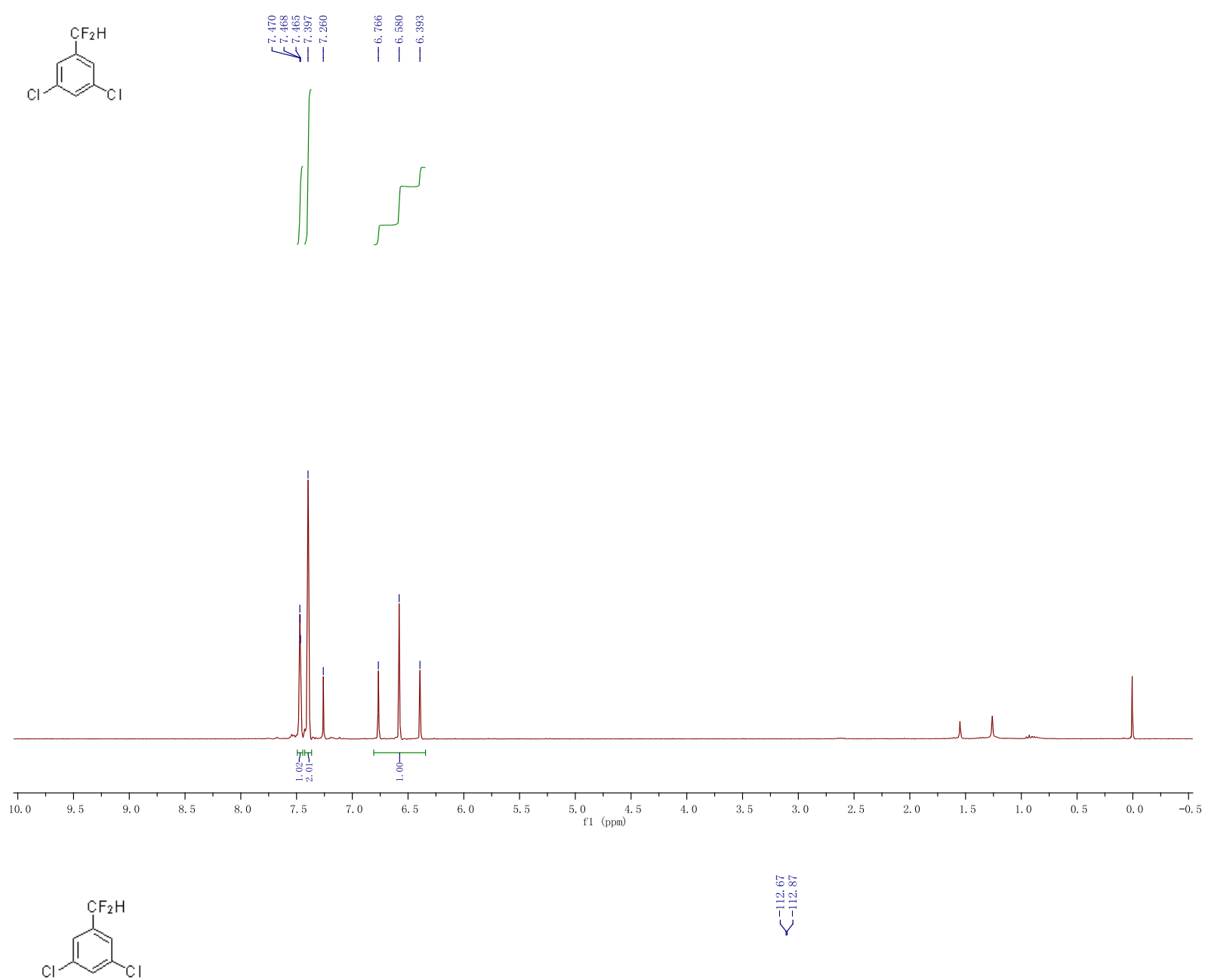

50
0
$0 \pm$
7
7

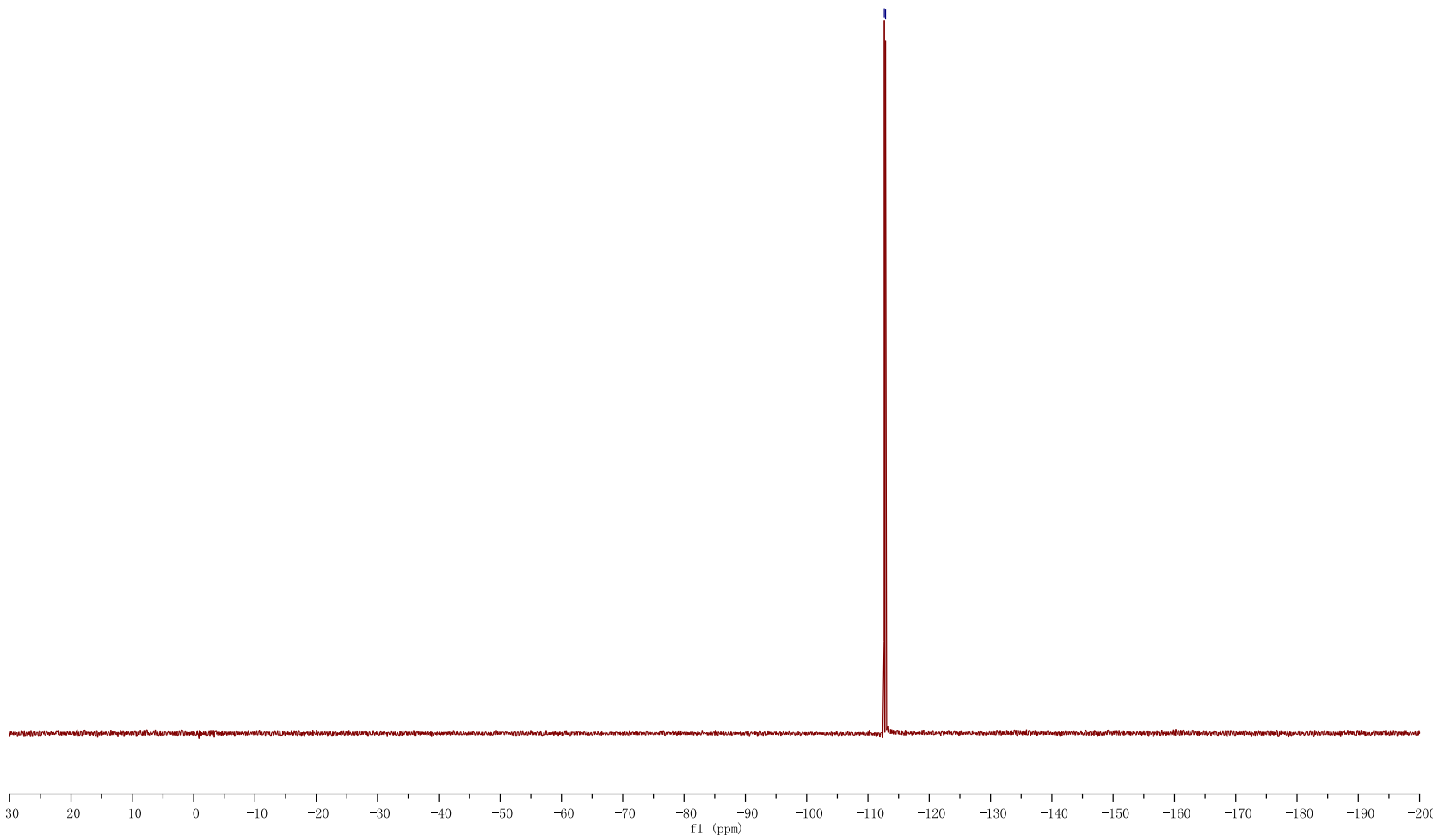


1-Bromo-2-(difluoromethyl)benzene (29).
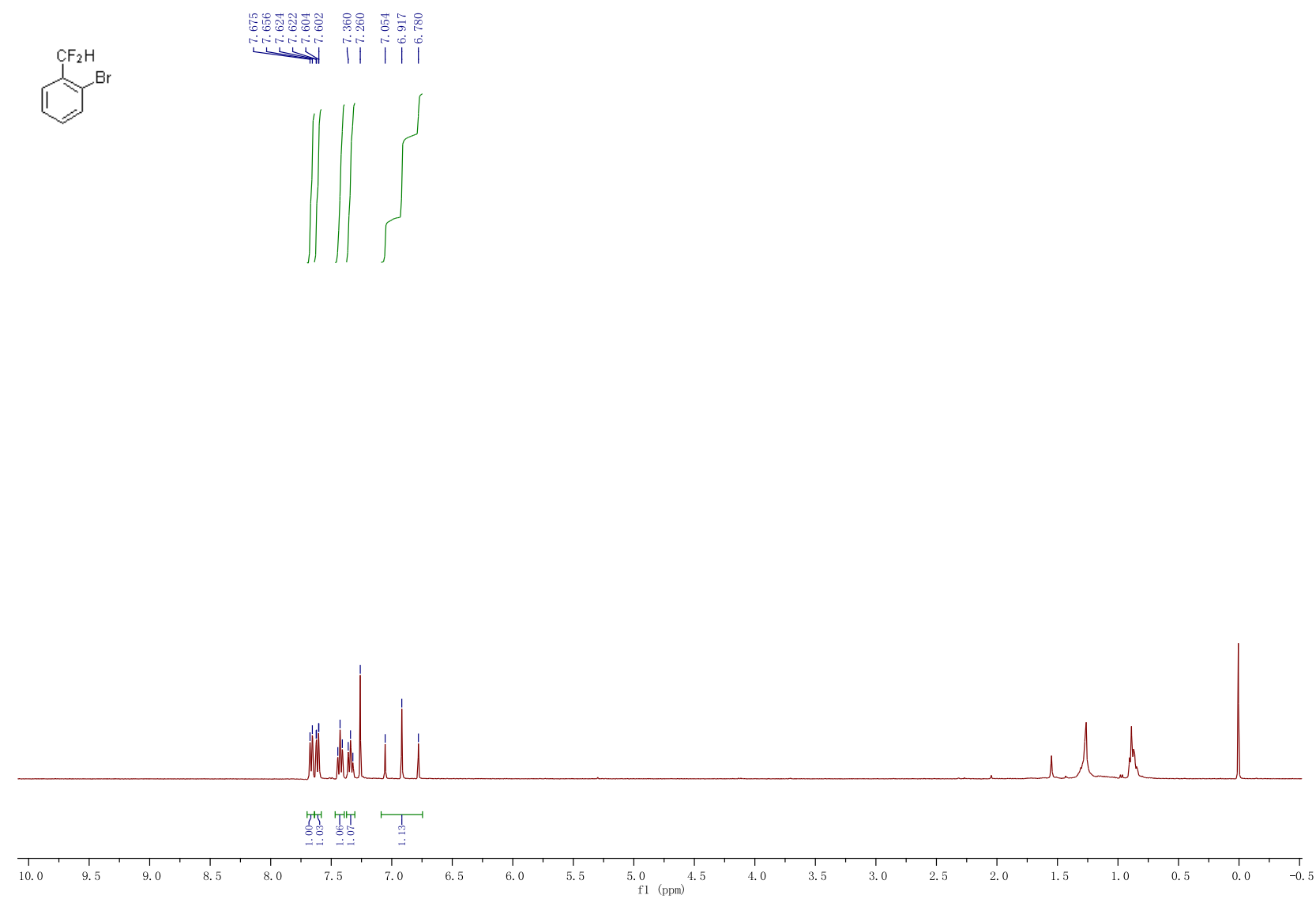

${ }^{\mathrm{Br}}$

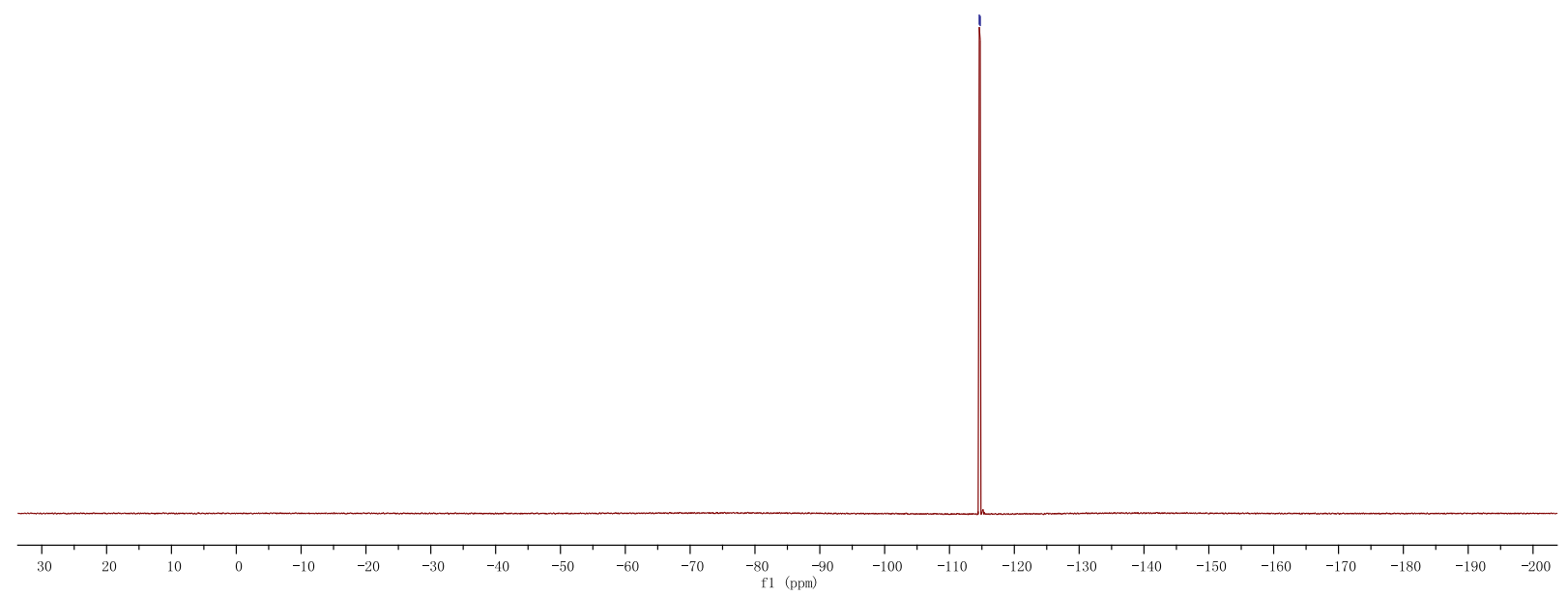


(4-(Difluoromethyl)phenyl)trimethylsilane (30).
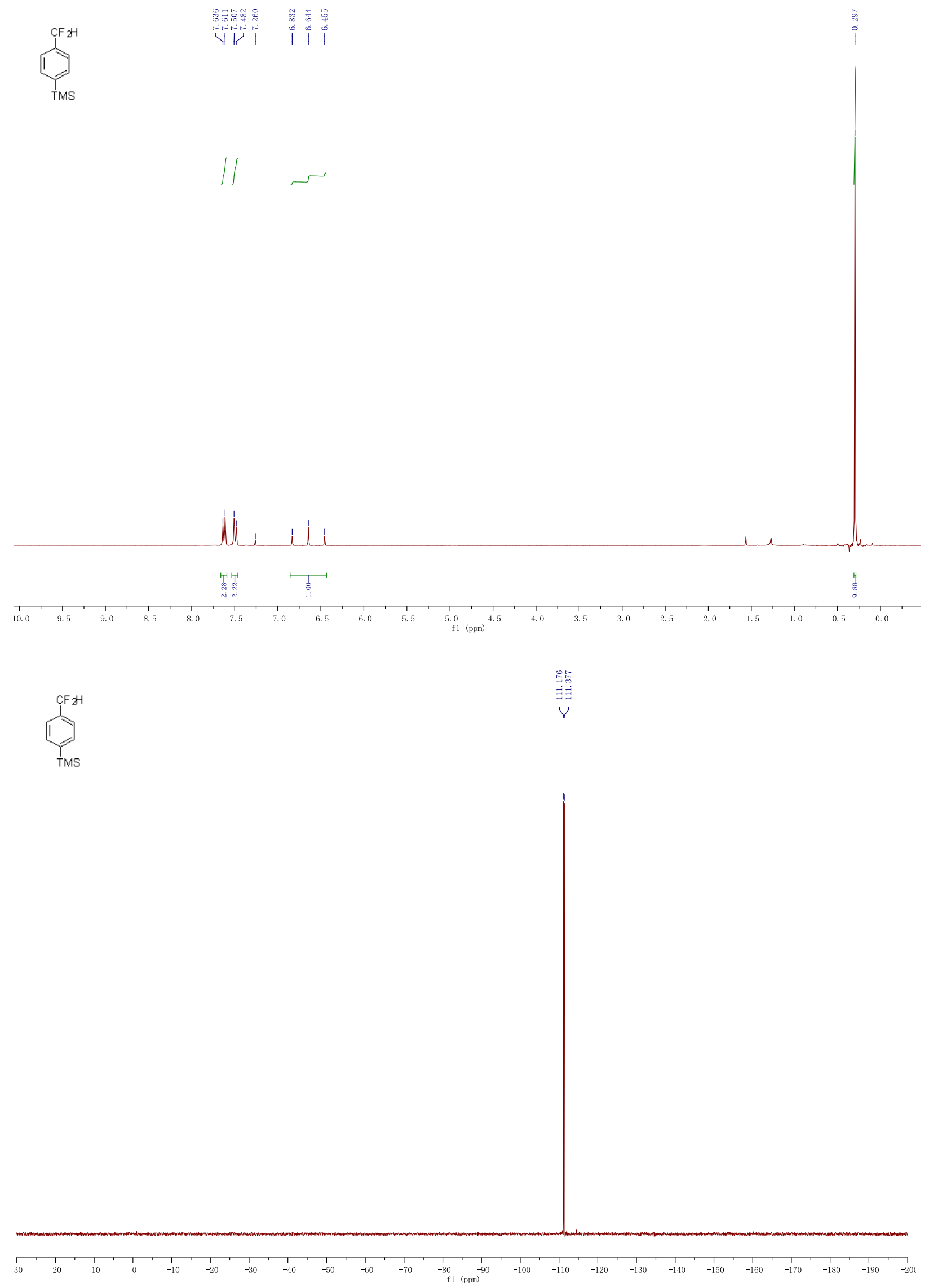
TMS

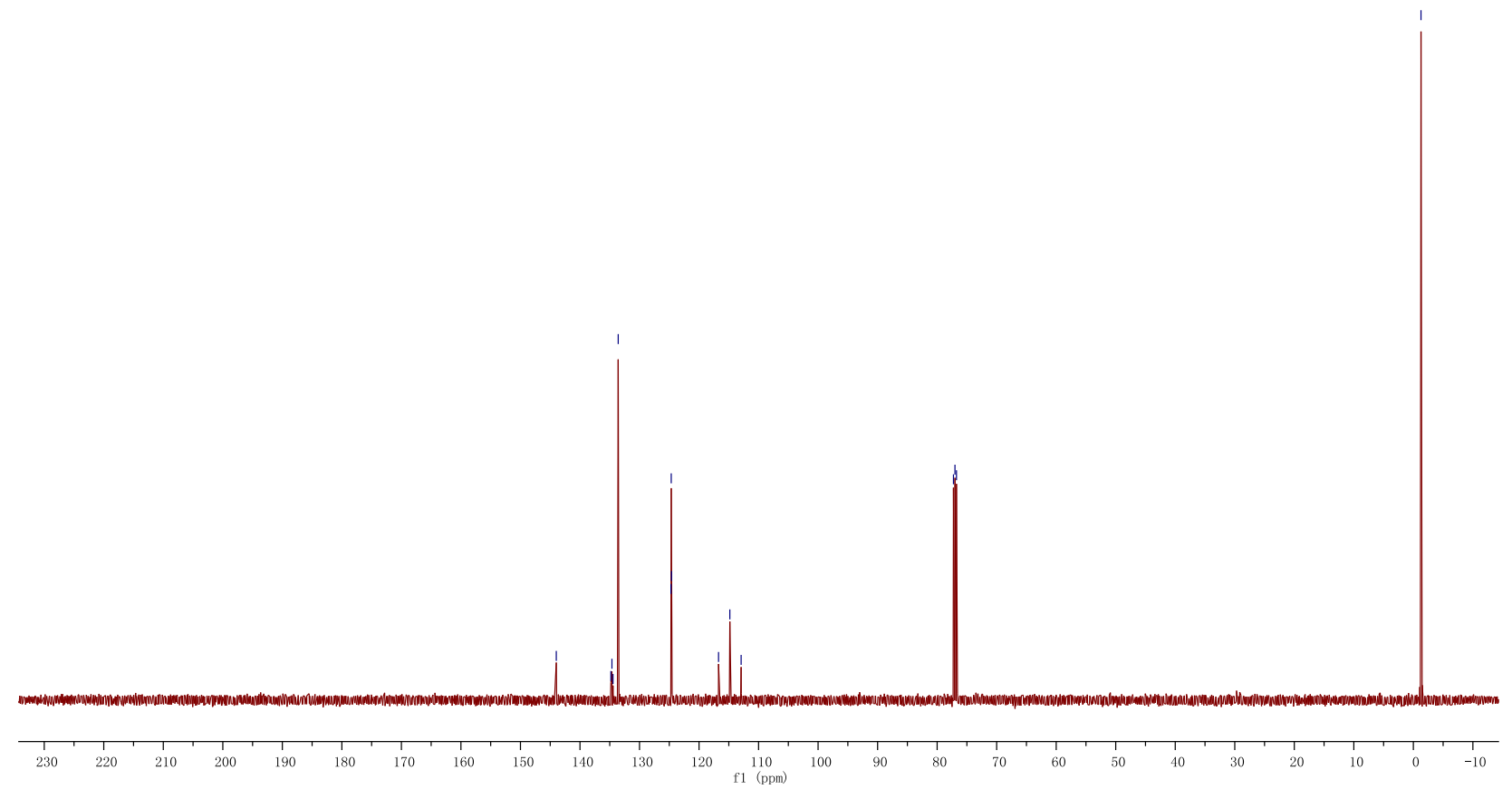

(4-(Difluoromethyl)phenyl)(ethyl)sulfane (31).
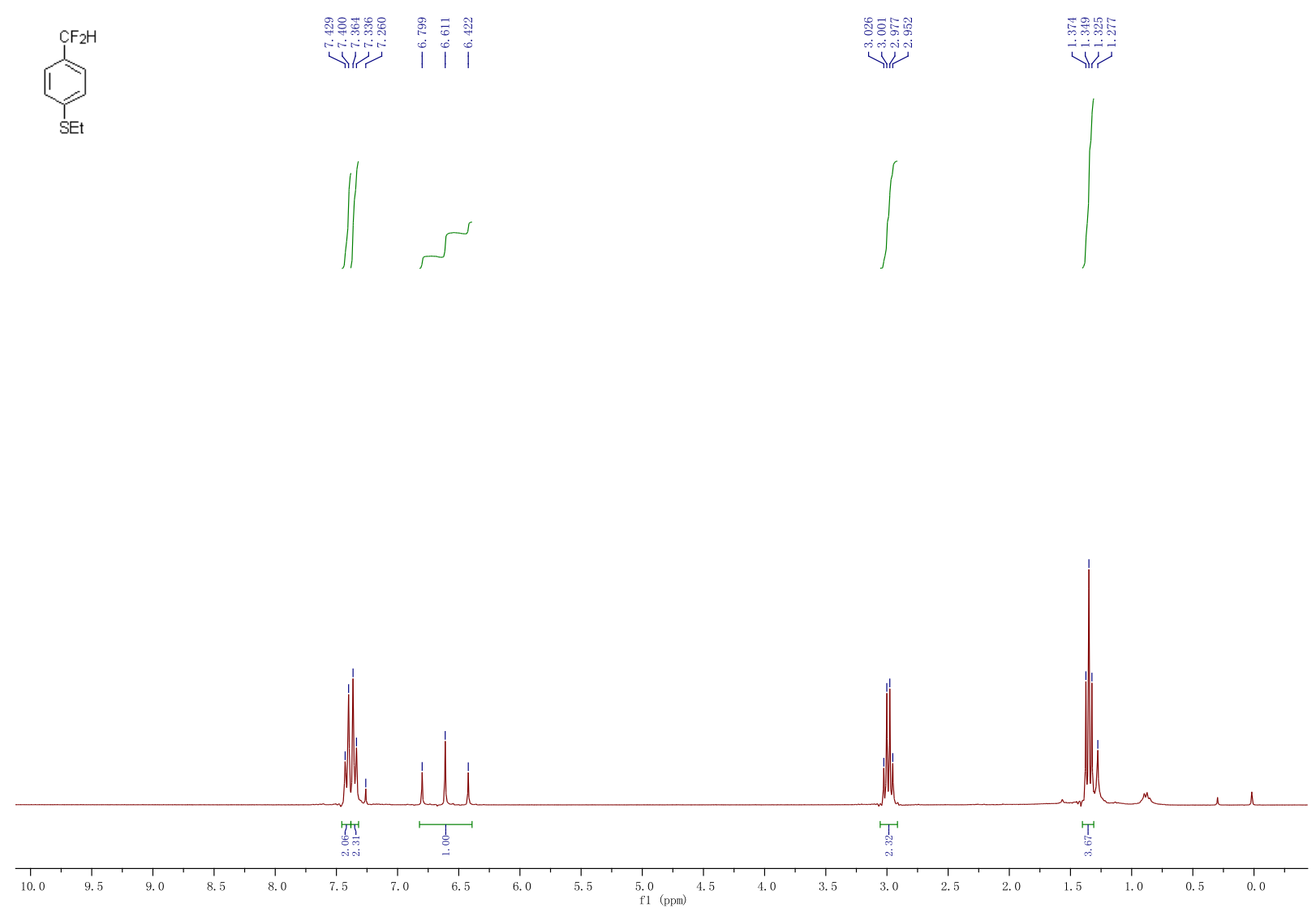
$\sum_{S E t}^{C F_{2} H}$
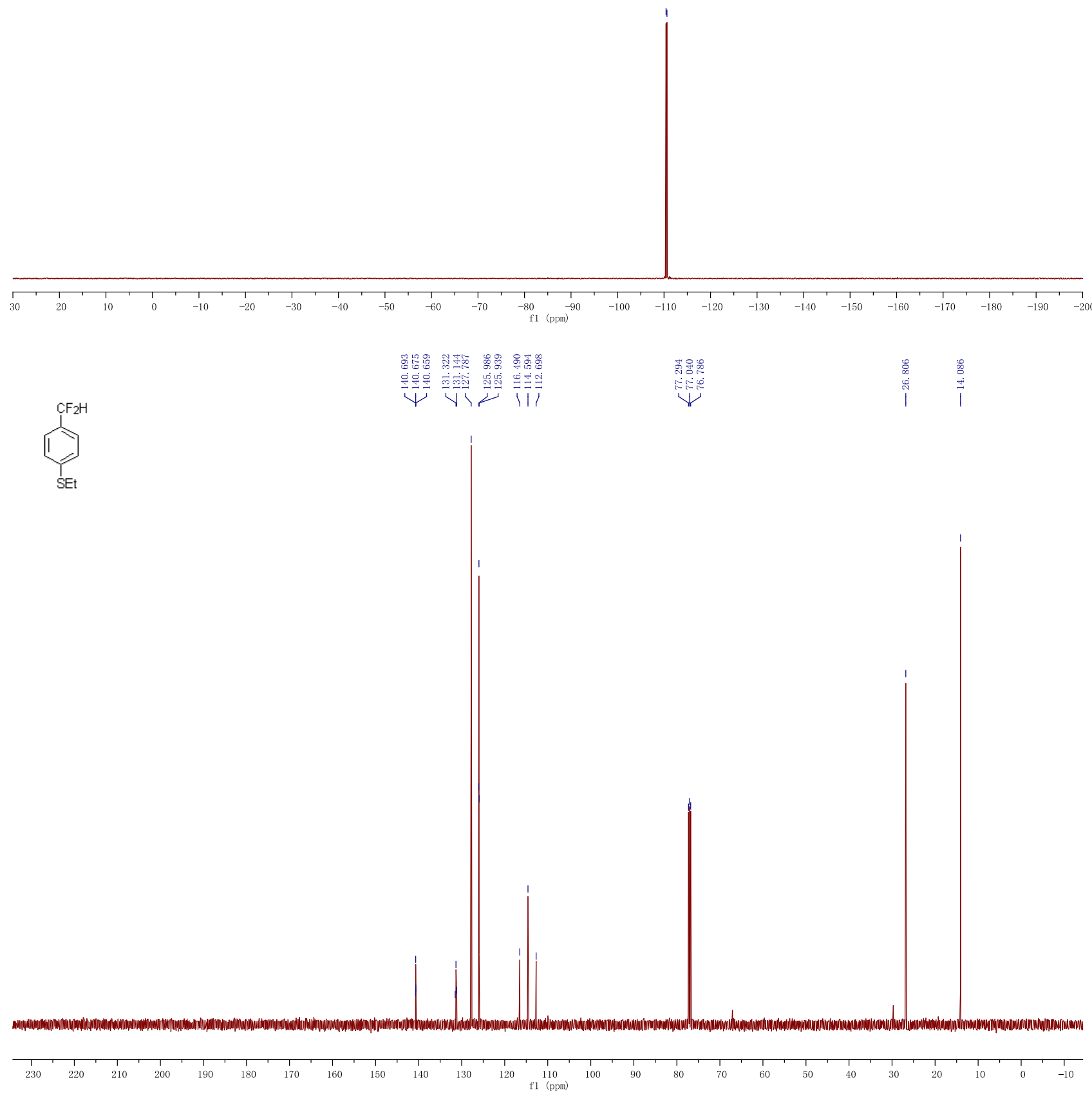
(4-(Difluoromethyl)phenyl)methanol (32).
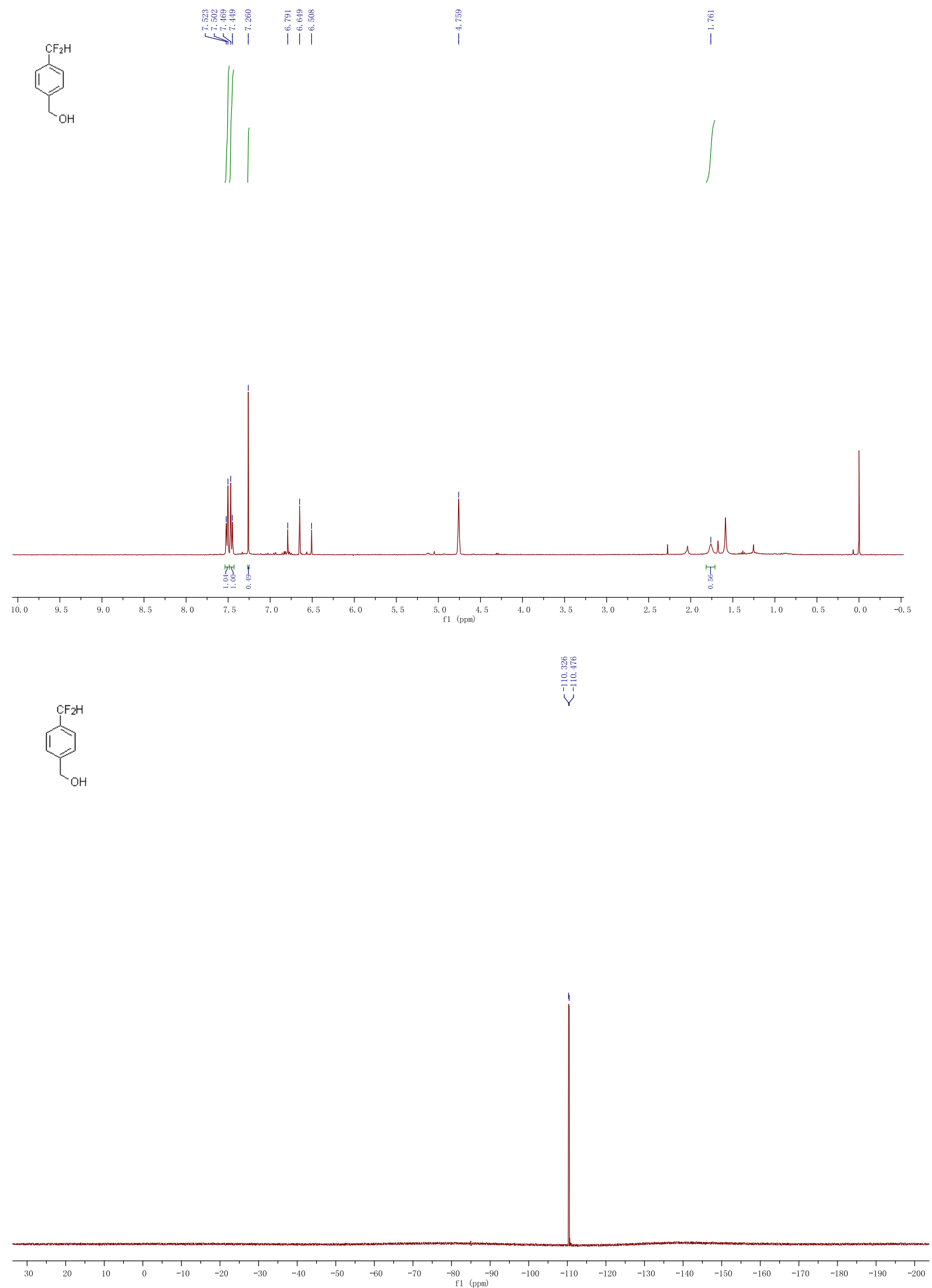
4-(Difluoromethyl)dibenzo[b,d]furan (33).
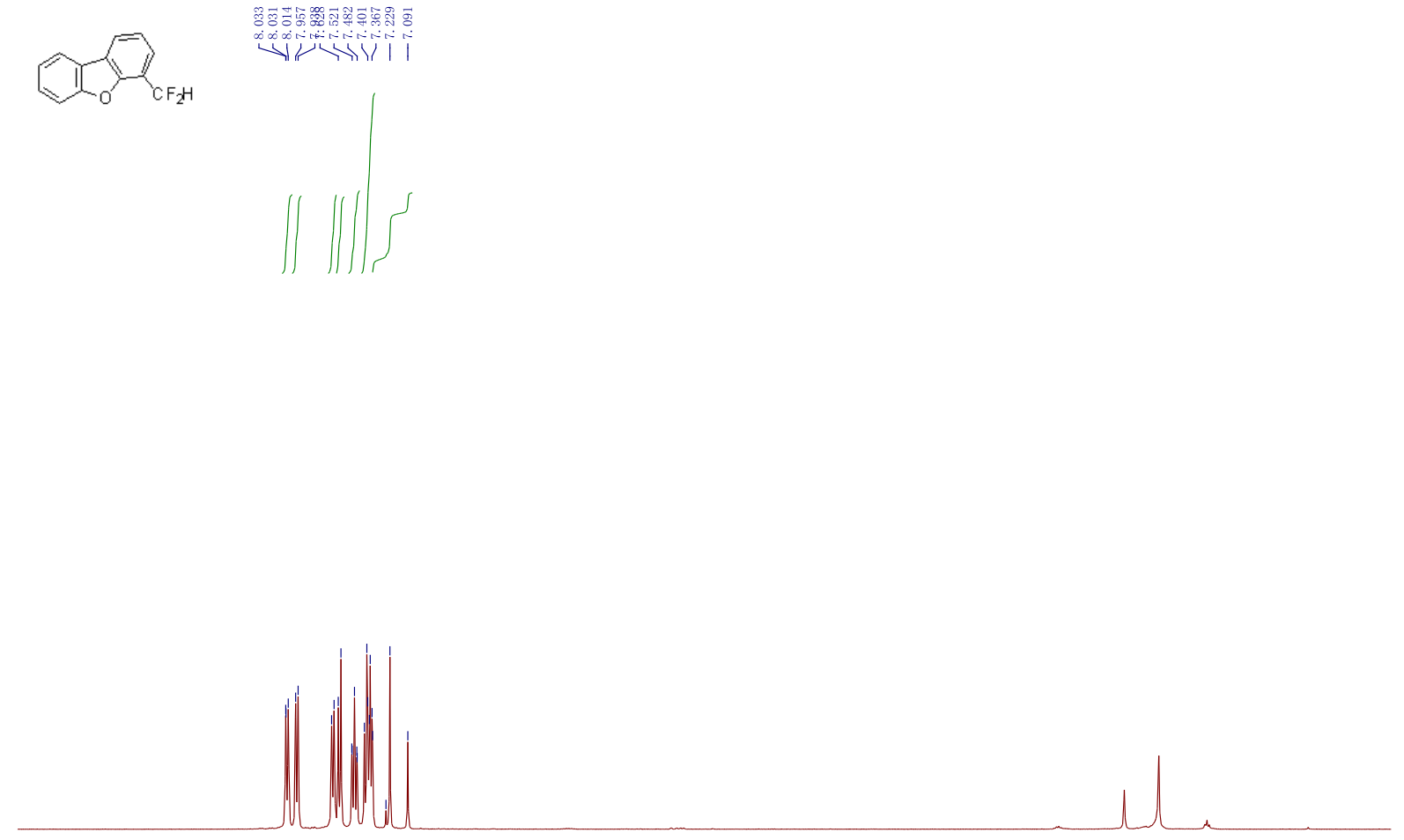

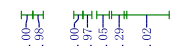
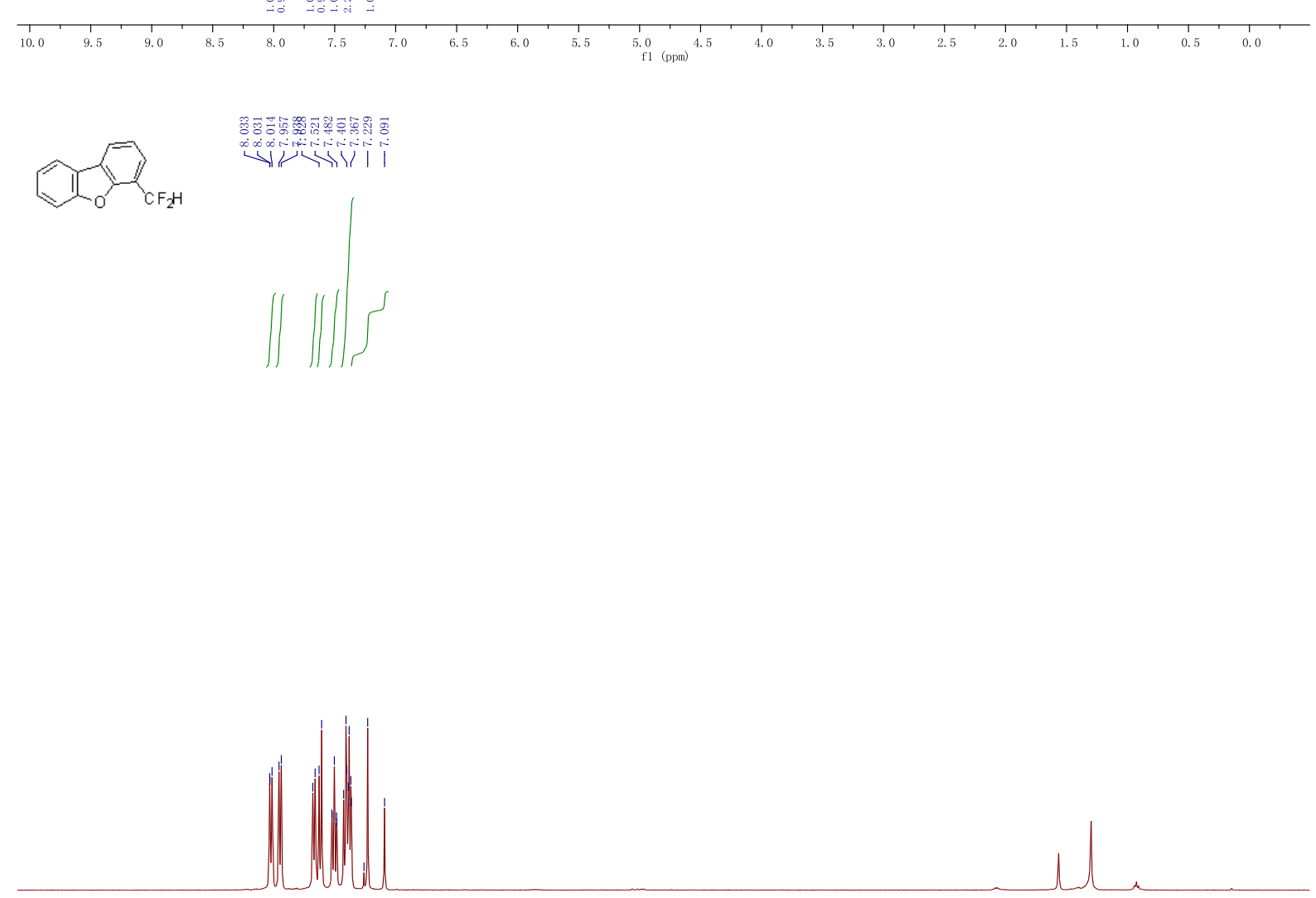

落茄

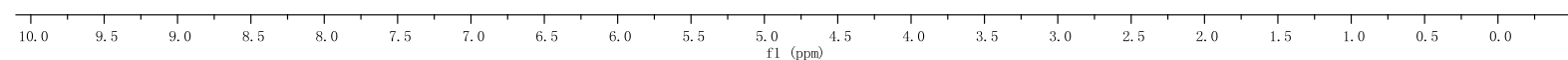



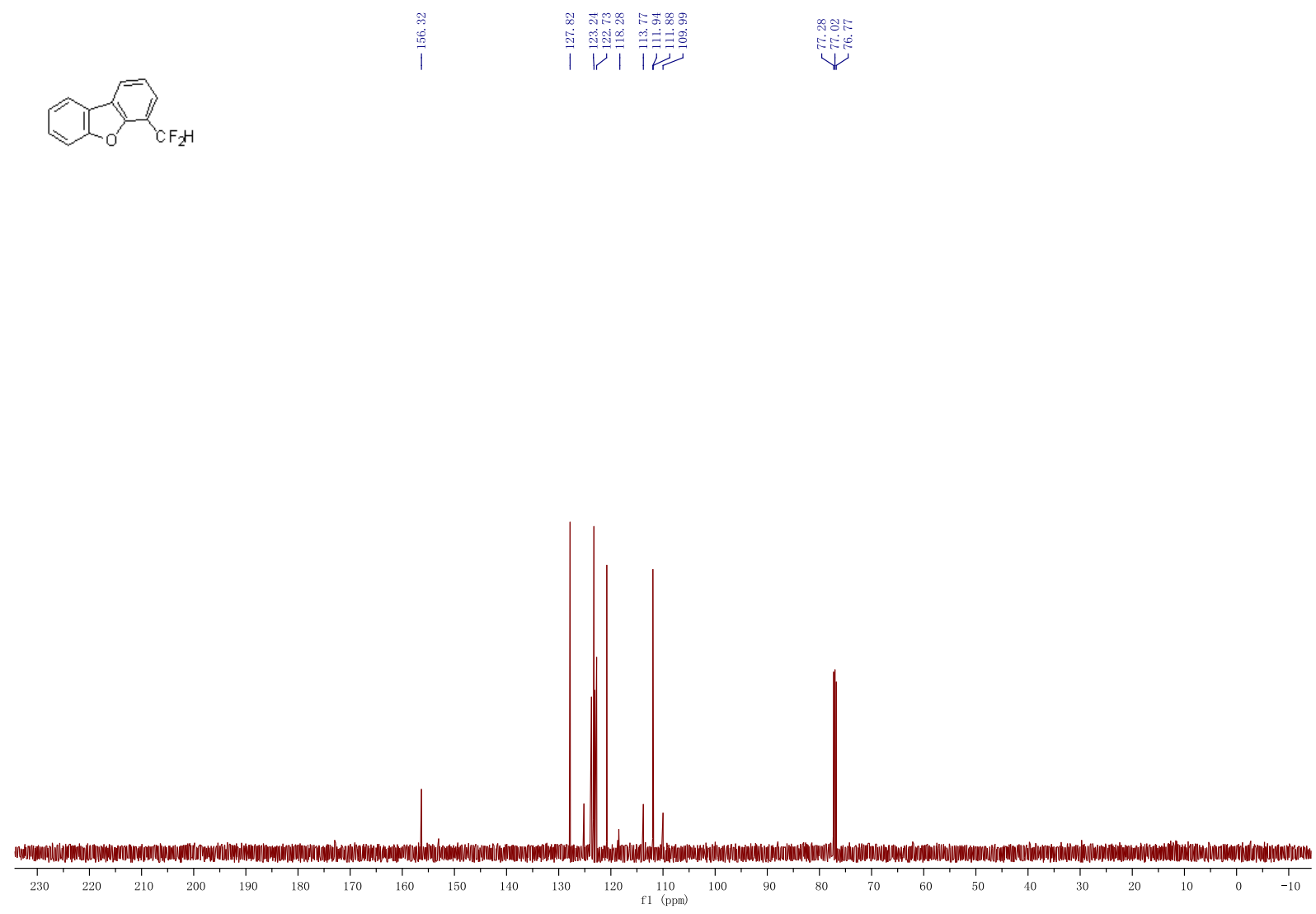

4-(Difluoromethyl)dibenzo[b,d]thiophene (34).
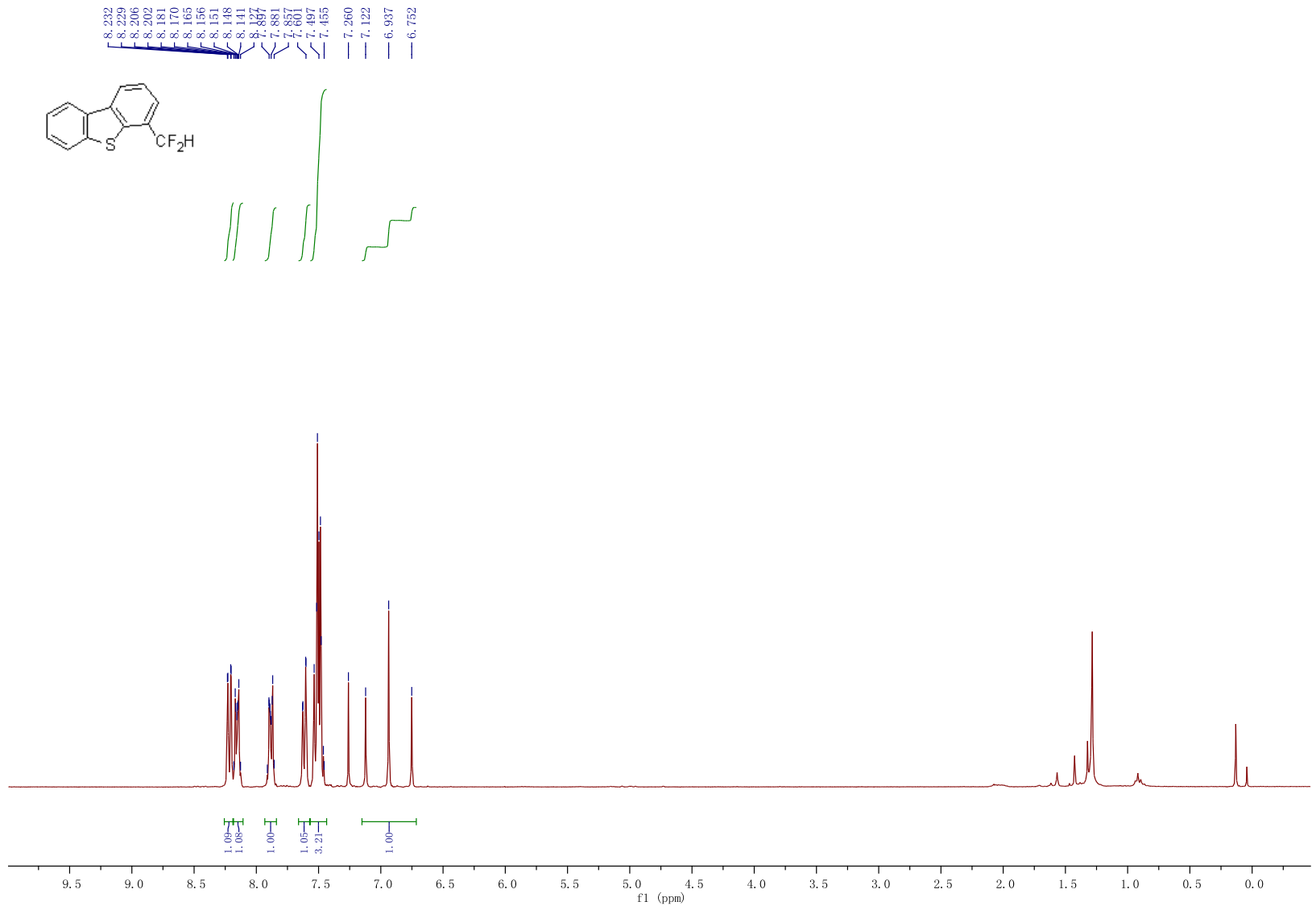

S80 


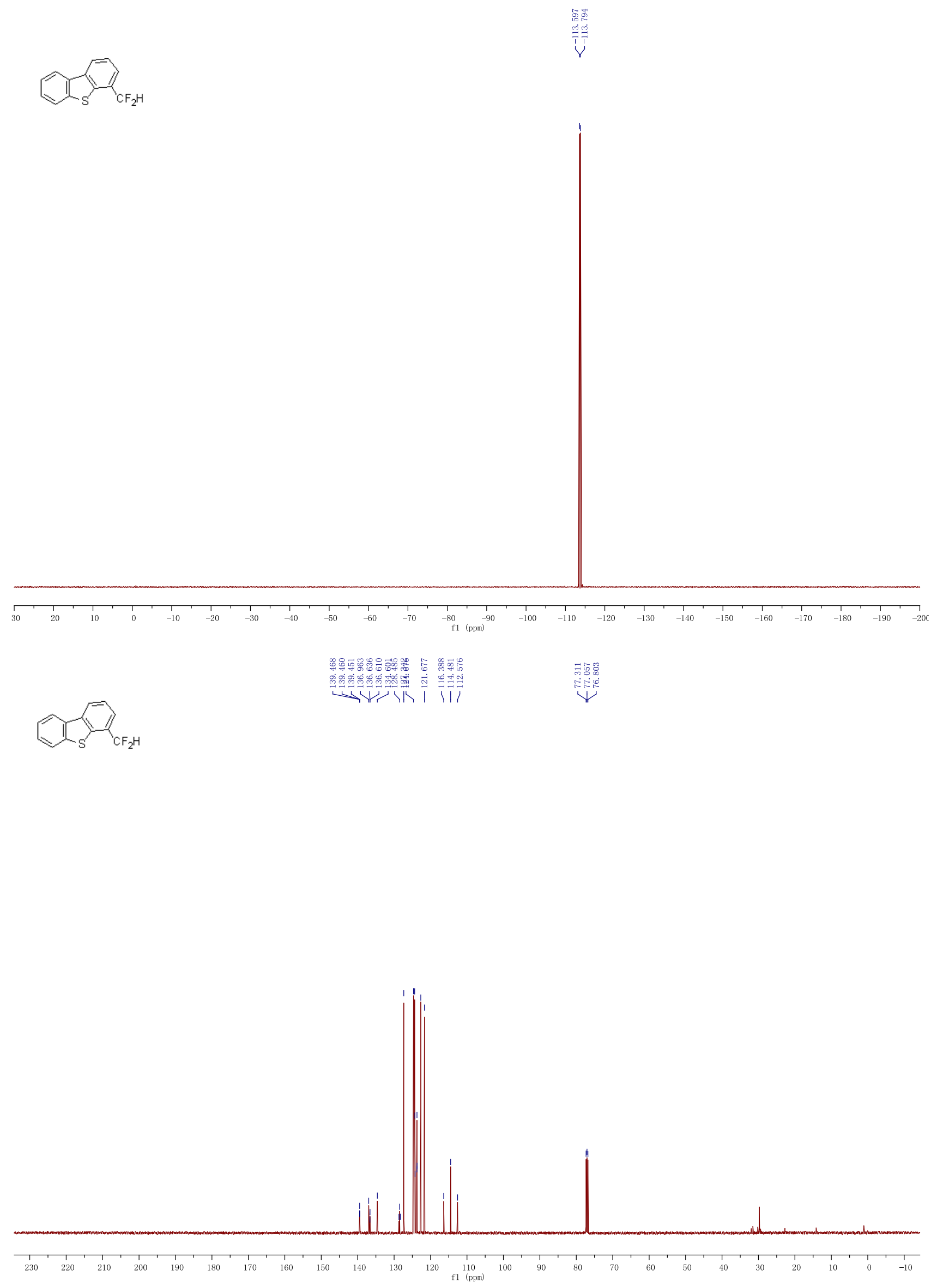


3-(Difluoromethyl)-9-phenyl-9H-carbazole (35).
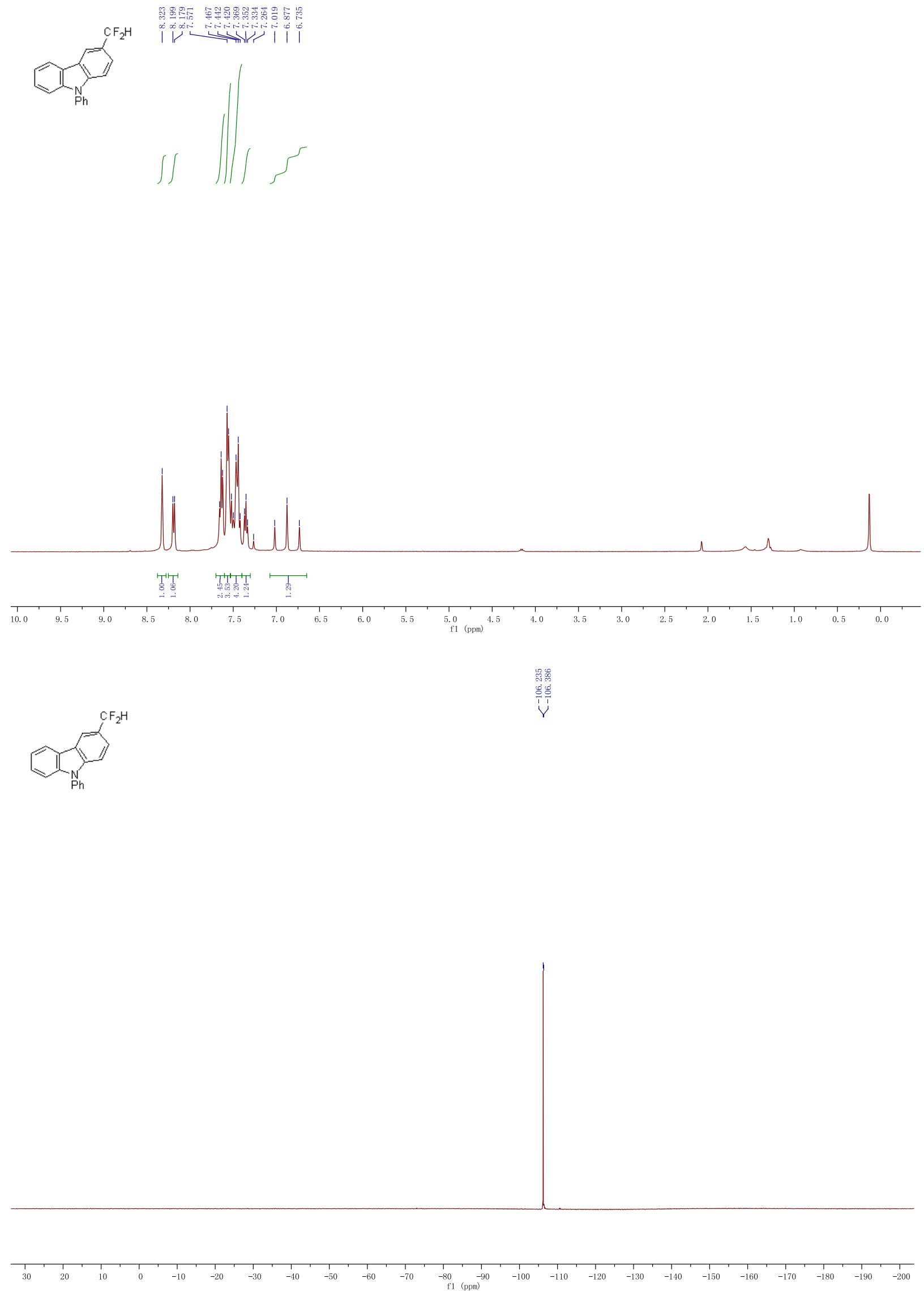


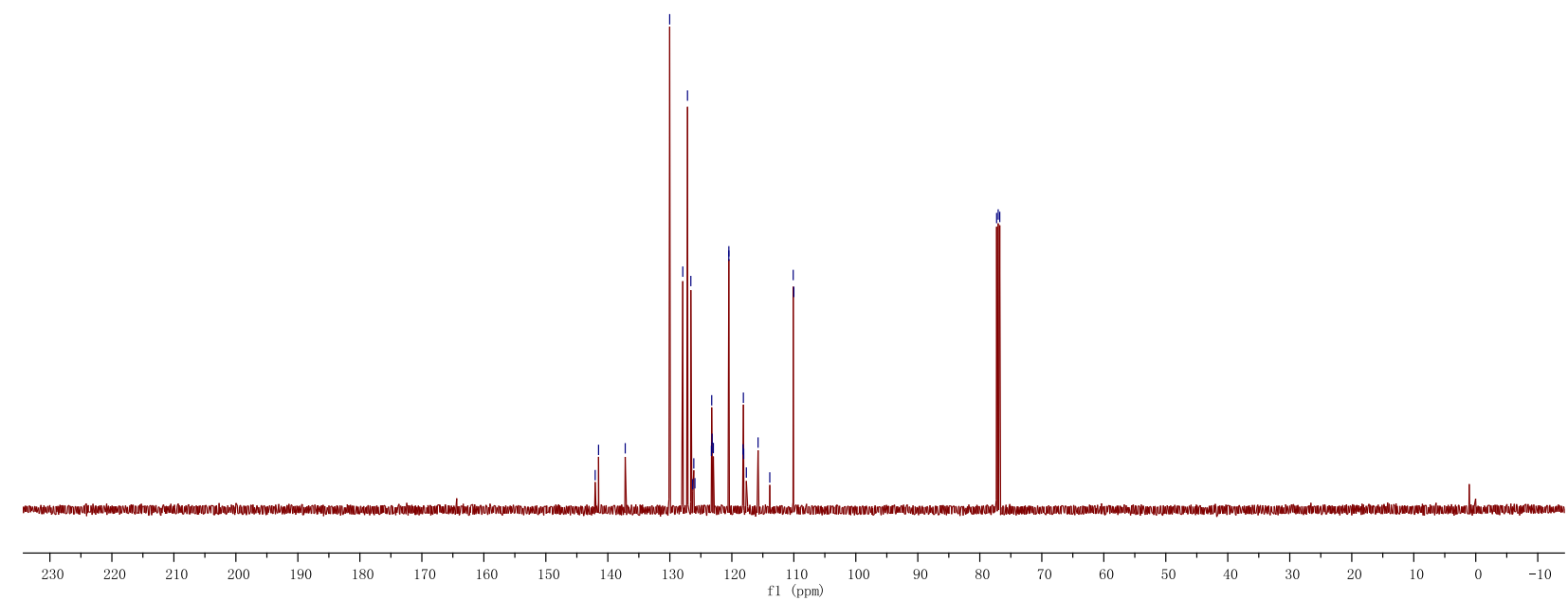

4-(4-(Difluoromethyl)phenyl)morpholine (36).
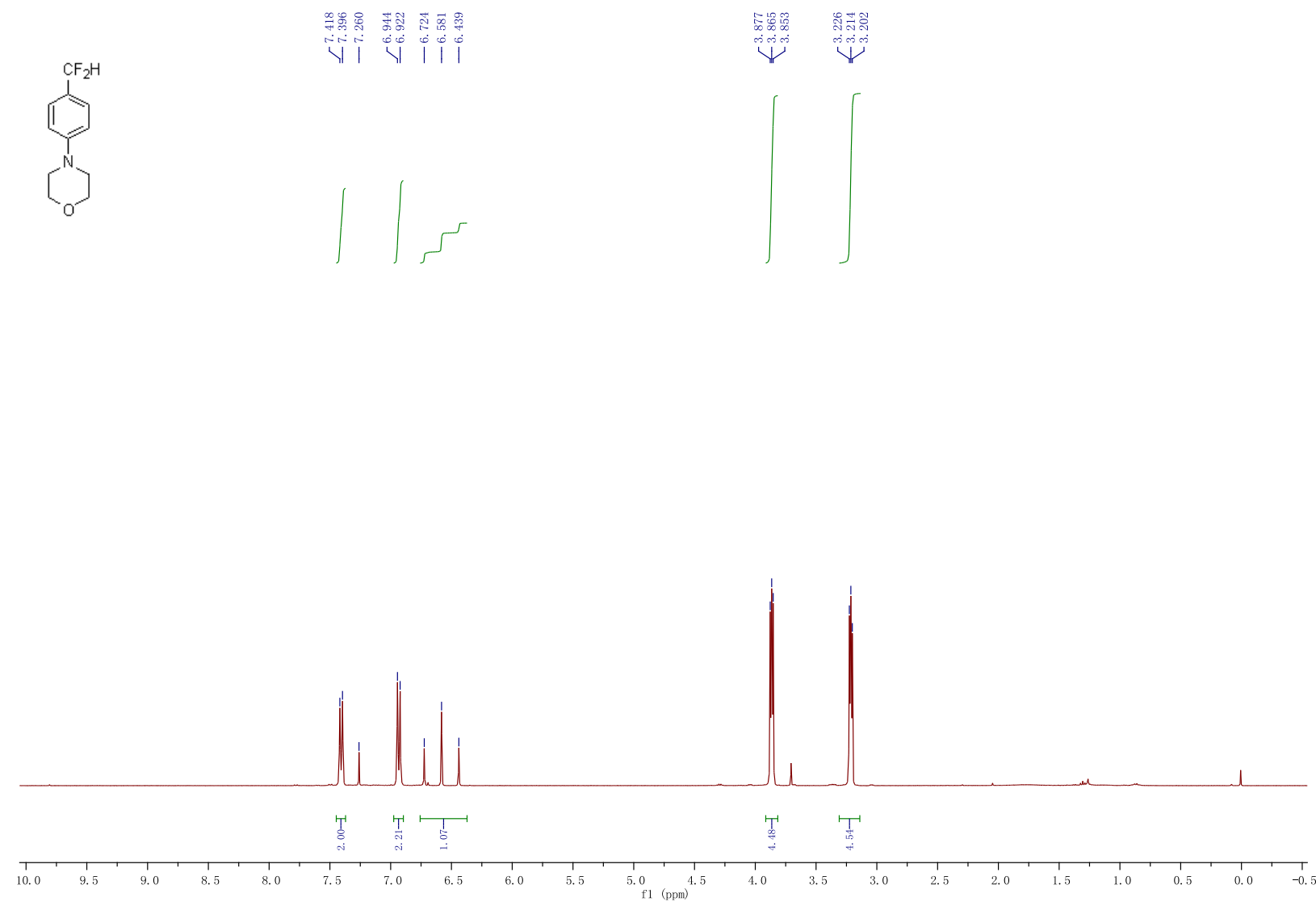
<smiles>[C+]c1ccc(N2CCOCC2)cc1</smiles>
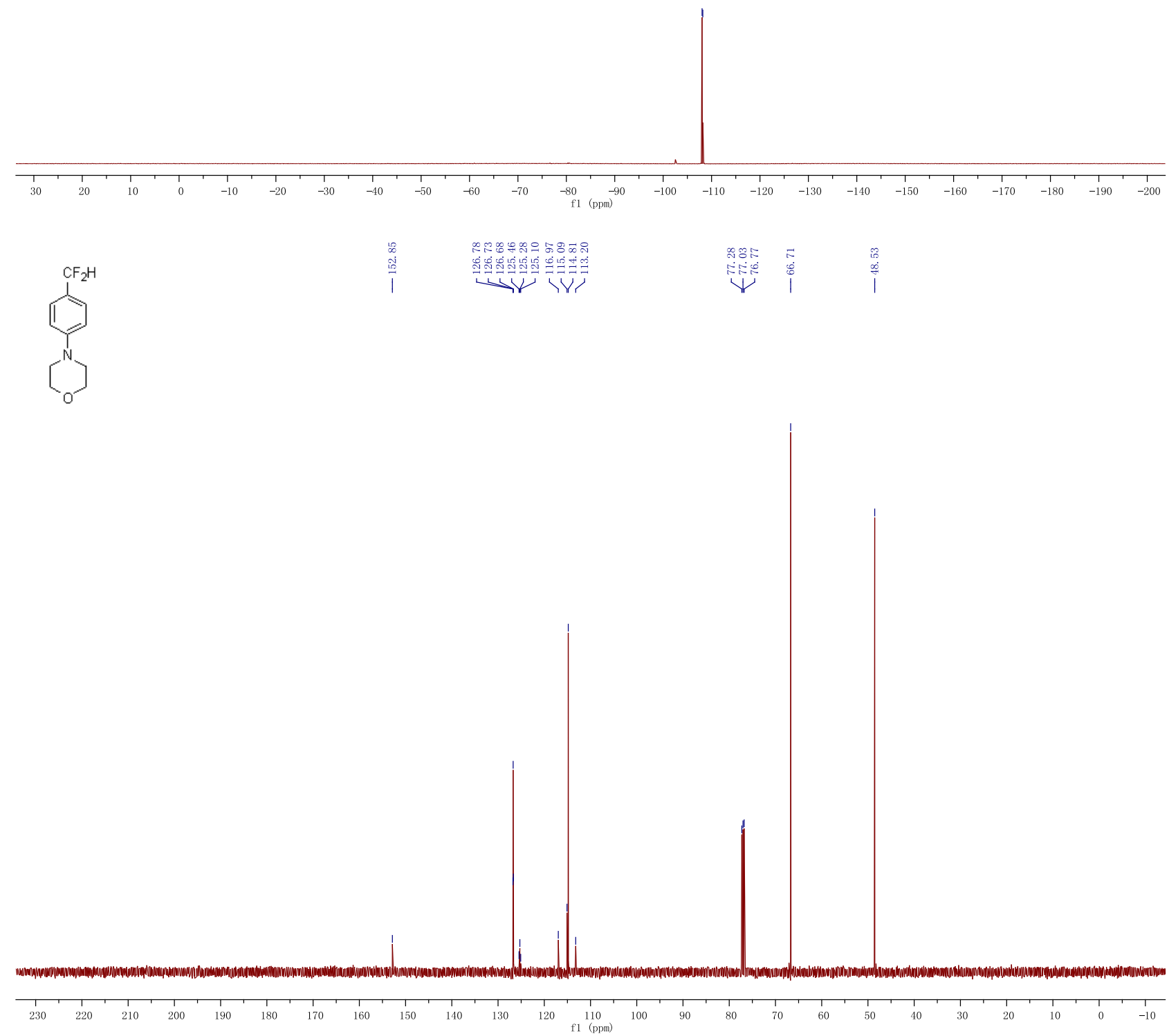
(2-(4-(Difluoromethyl)phenyl)chroman-4-one (37).
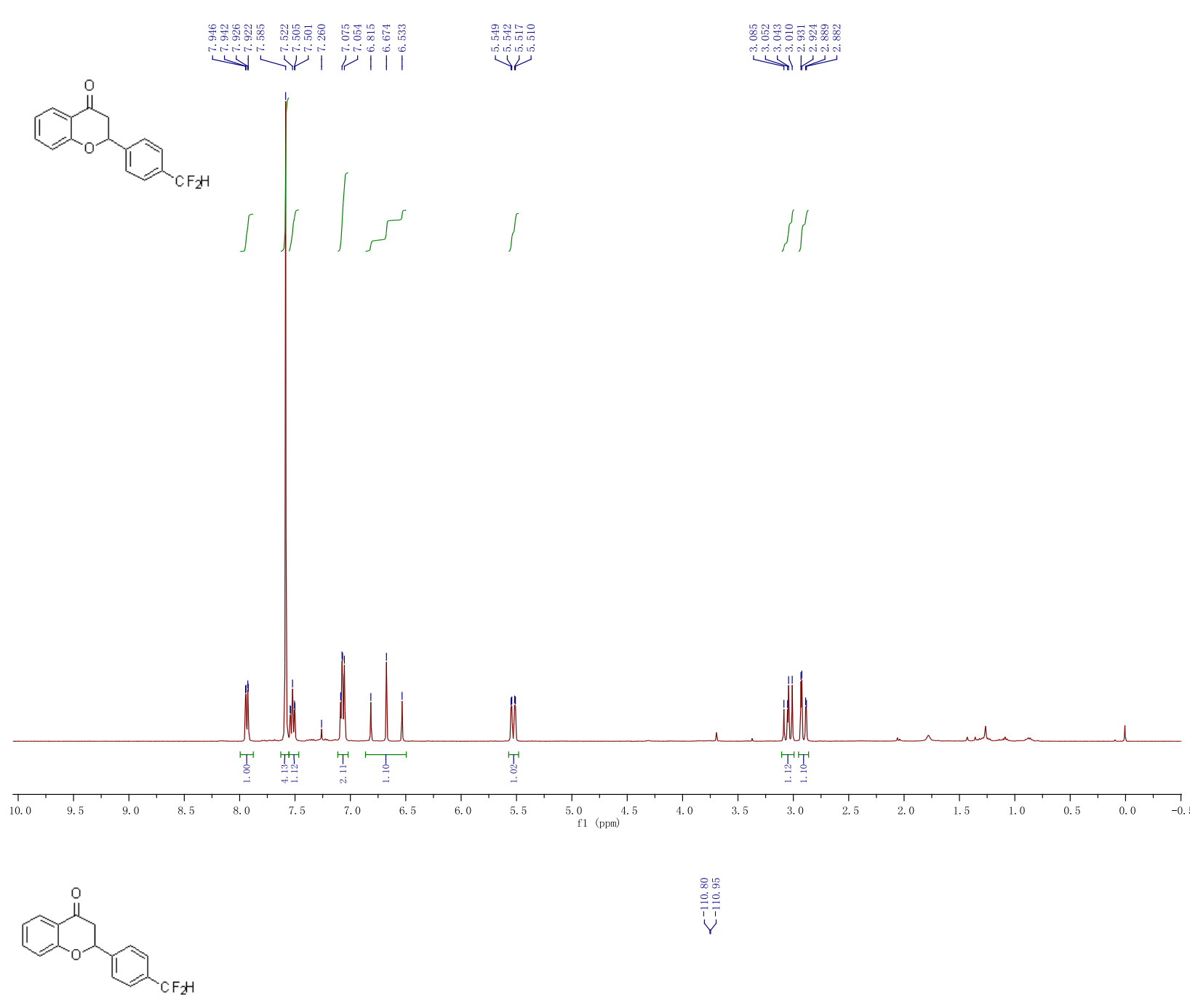

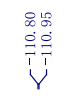

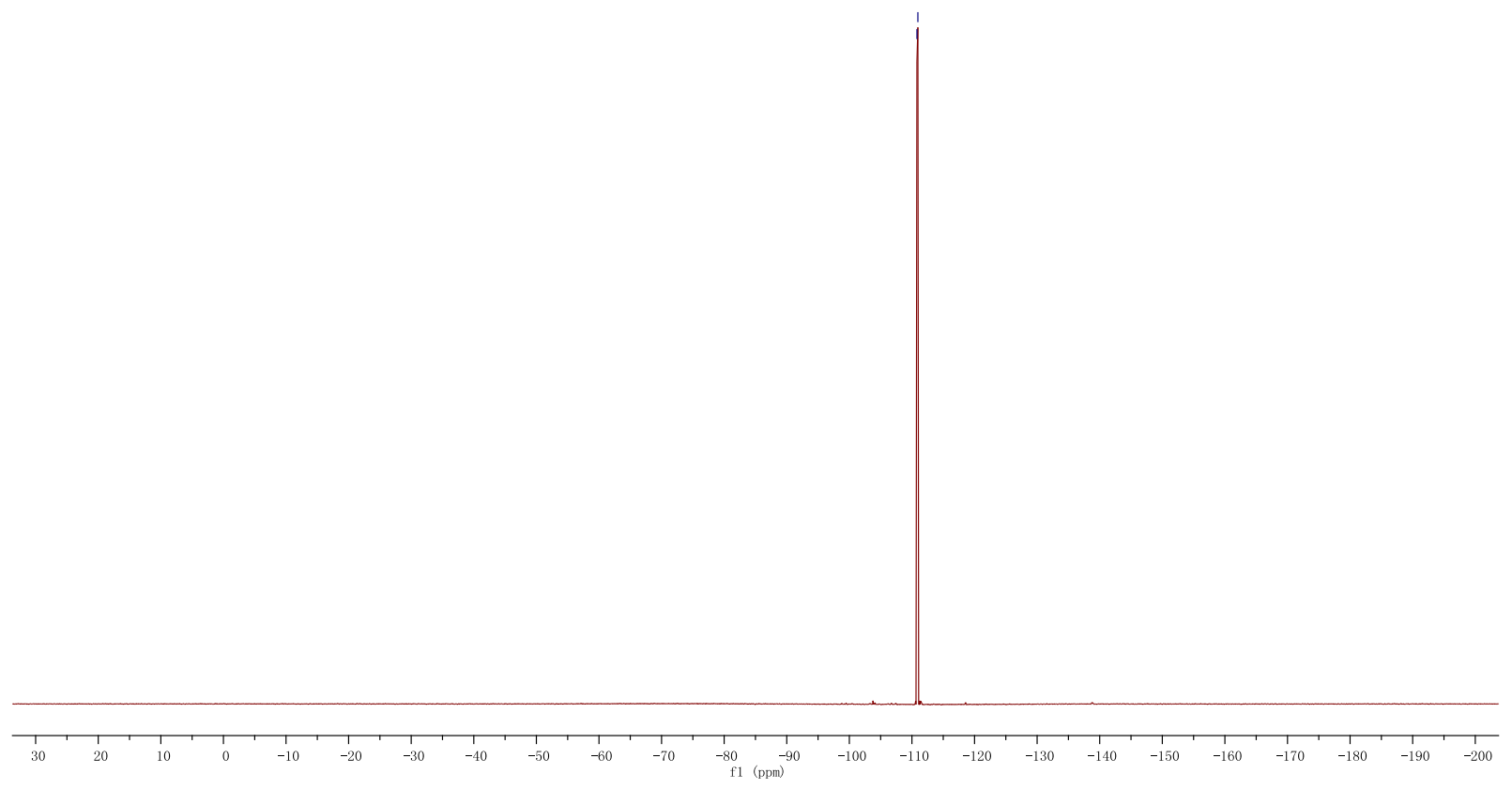

S85 
路的

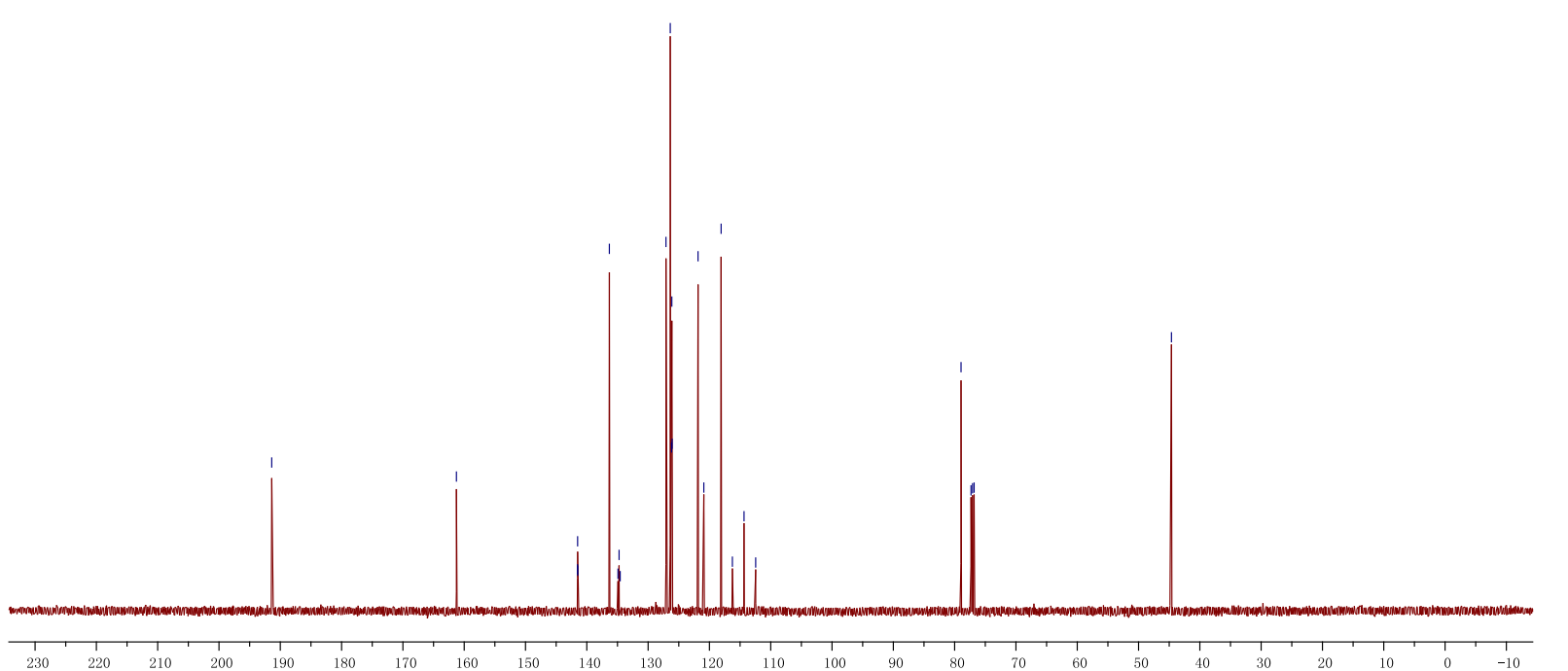

(S)-Methyl 2-((tert-butoxycarbonyl)amino)-3-(4-(difluoromethyl)phenyl)propanoate (38).

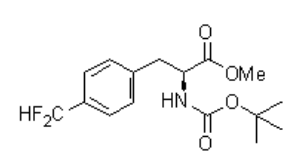

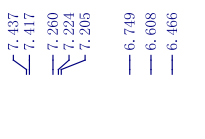

新吉

II

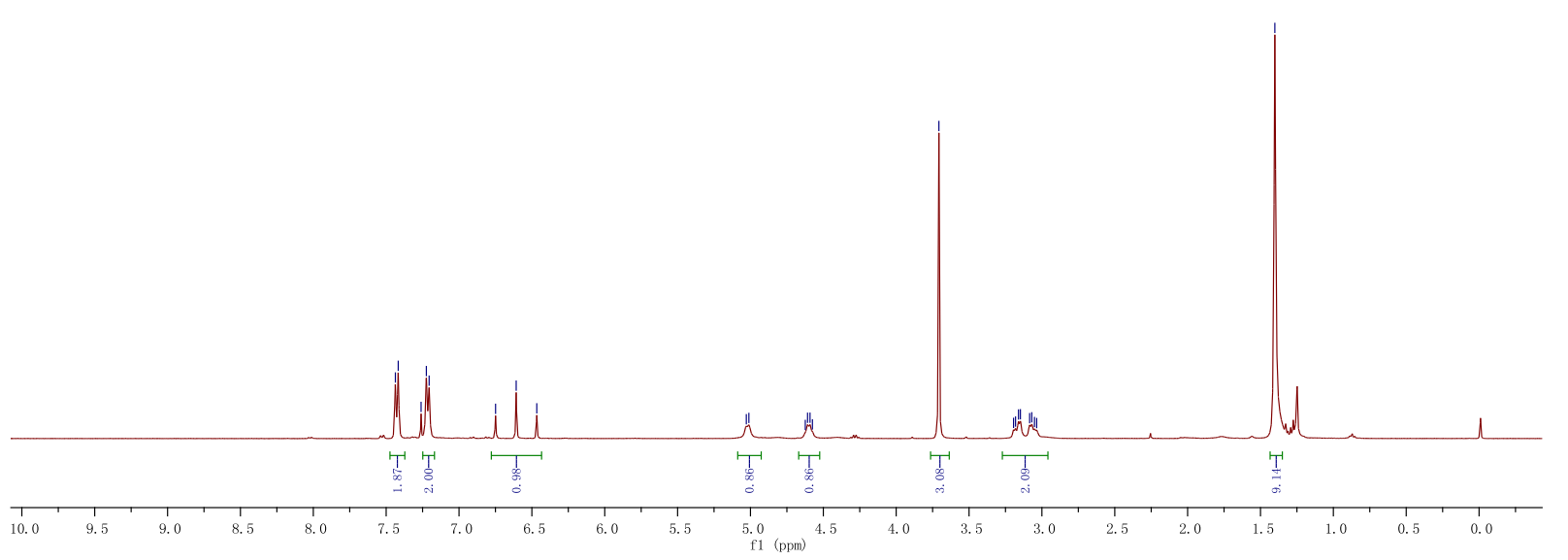

S86 


$$
\text { 而 }
$$
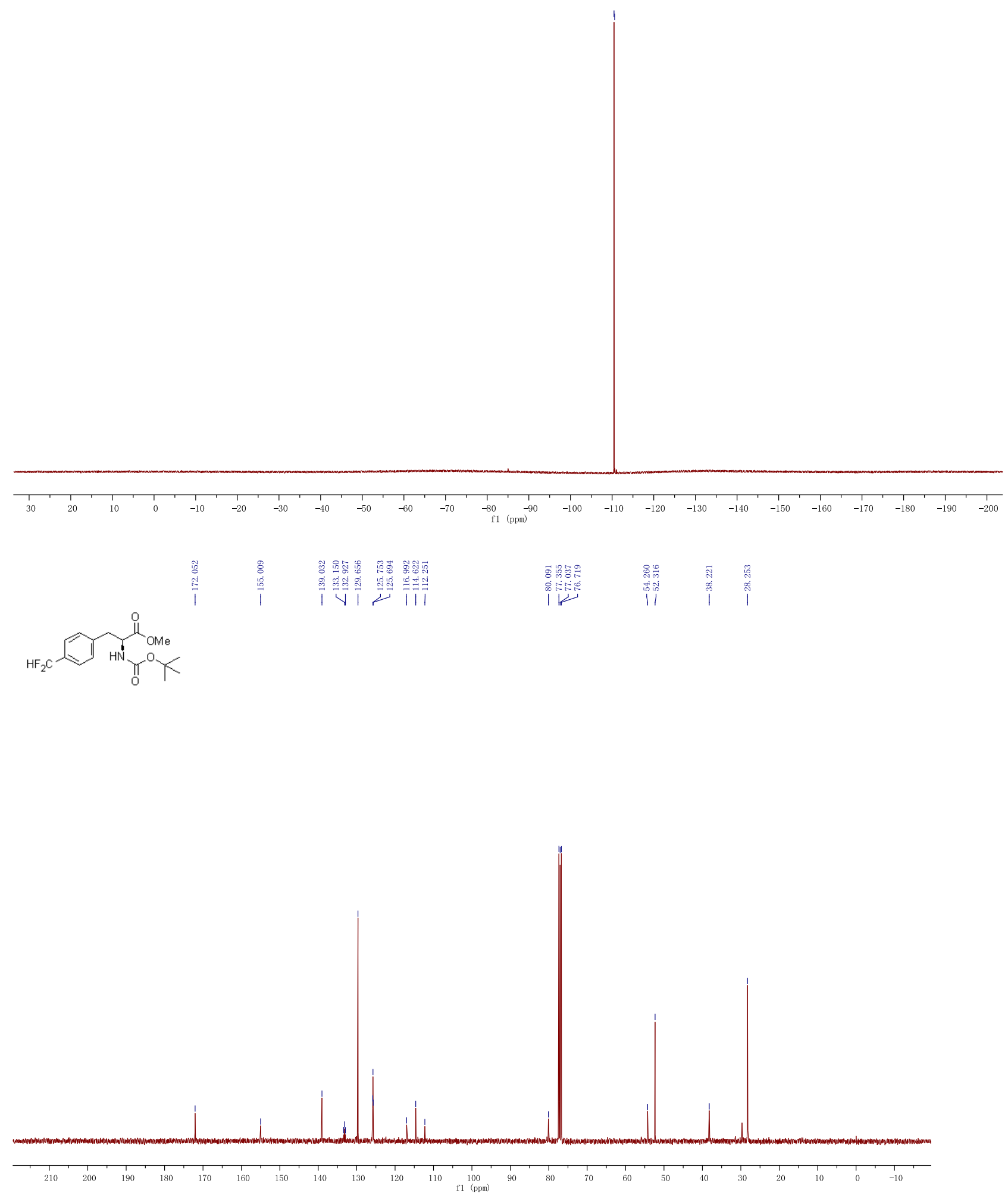
(3R)-4-(4-(Difluoromethyl)phenyl)-1-(4-fluorophenyl)-3-((S)-3-(4-fluorophenyl)-3hydroxypropyl)azetidin-2-one (39).
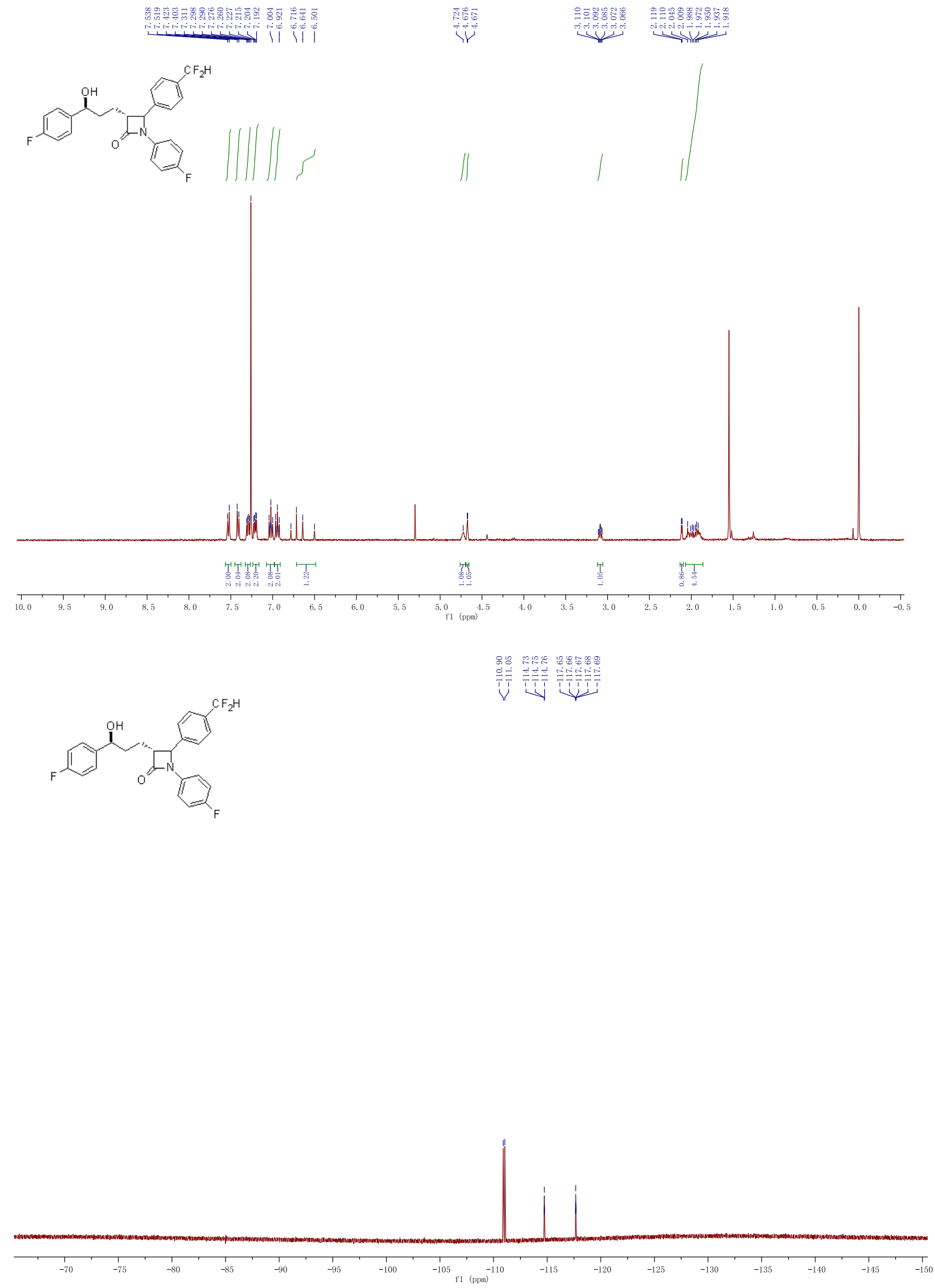


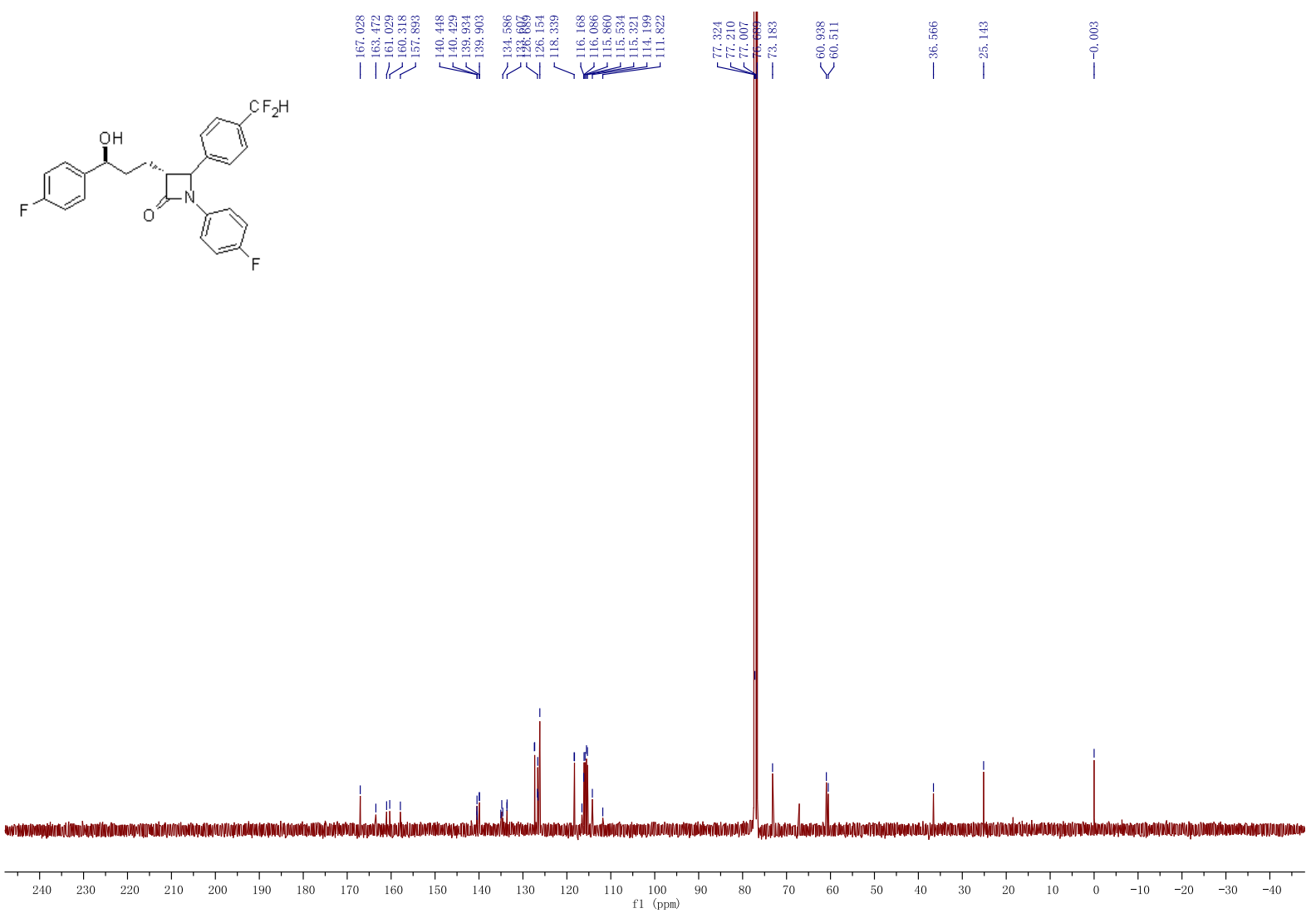

$(8 R, 9 S, 13 S, 14 S)-3-(D i f l u o r o m e t h y l)-13-m e t h y l-7,8,9,11,12,13,15,16-o c t a h y d r o-6 H$ -

cyclopenta $[a]$ phenanthren-17(14H)-one (40).

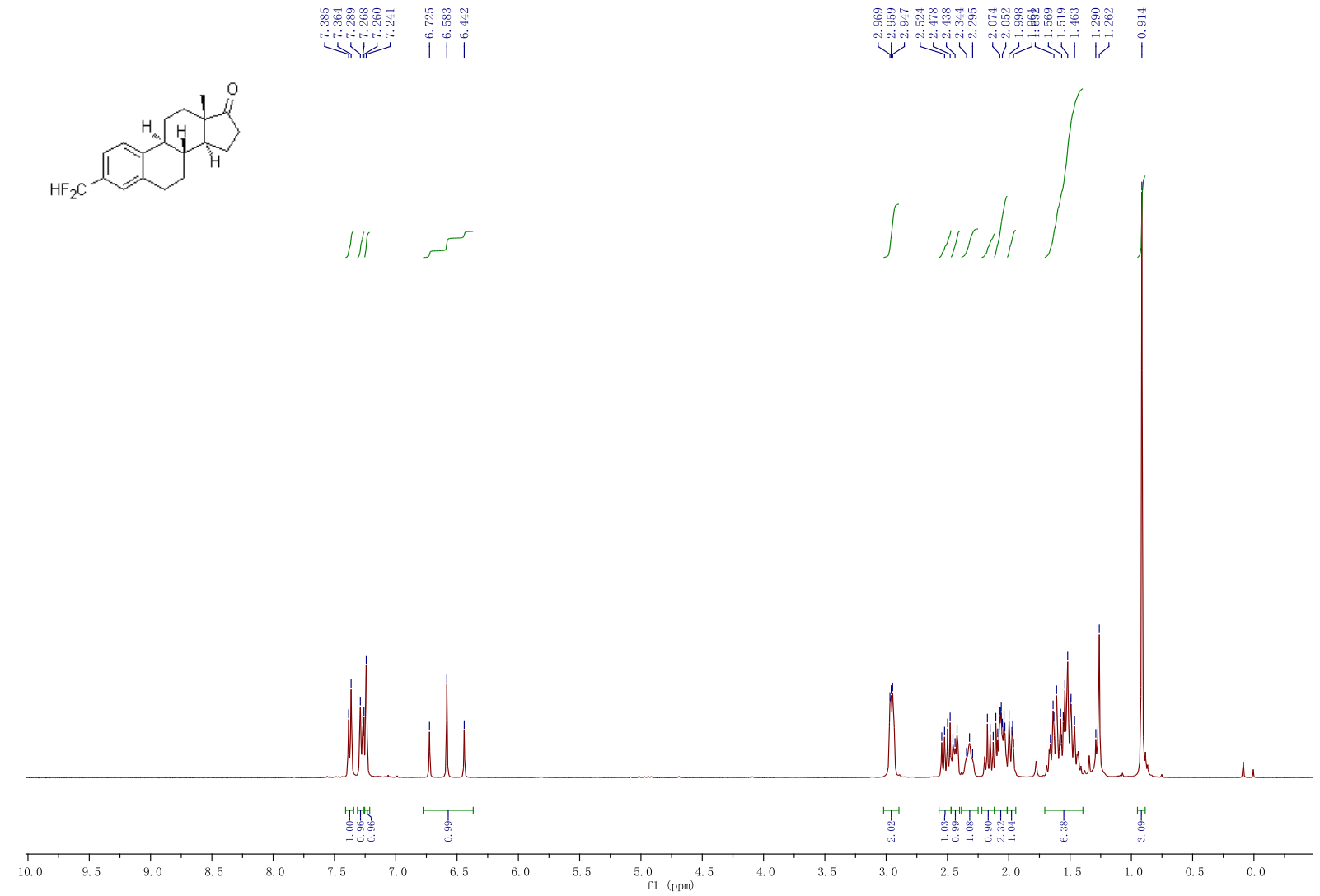


(n)
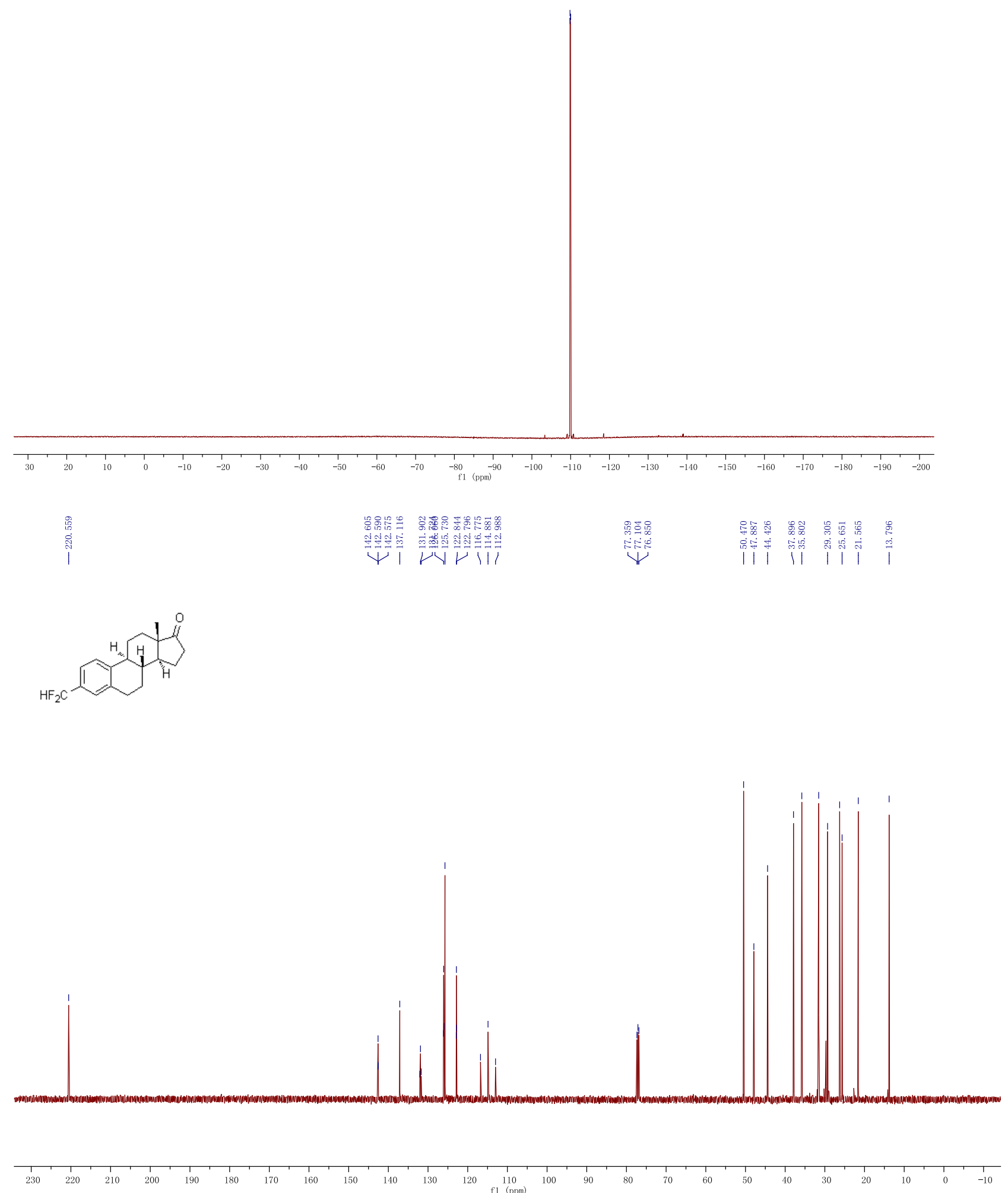